US Army Corps of Engineers ${ }_{\circledast}$ Engineer Research and Development Center

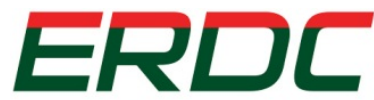

INNOVATIVE SOLUTIONS for a safer, better world

\title{
Geophysical Surveys for Locating Buried Utilities, Lake Pontchartrain Levees, New Orleans
}

José L. Llopis, Janet E. Simms, and Grant A. Riddick

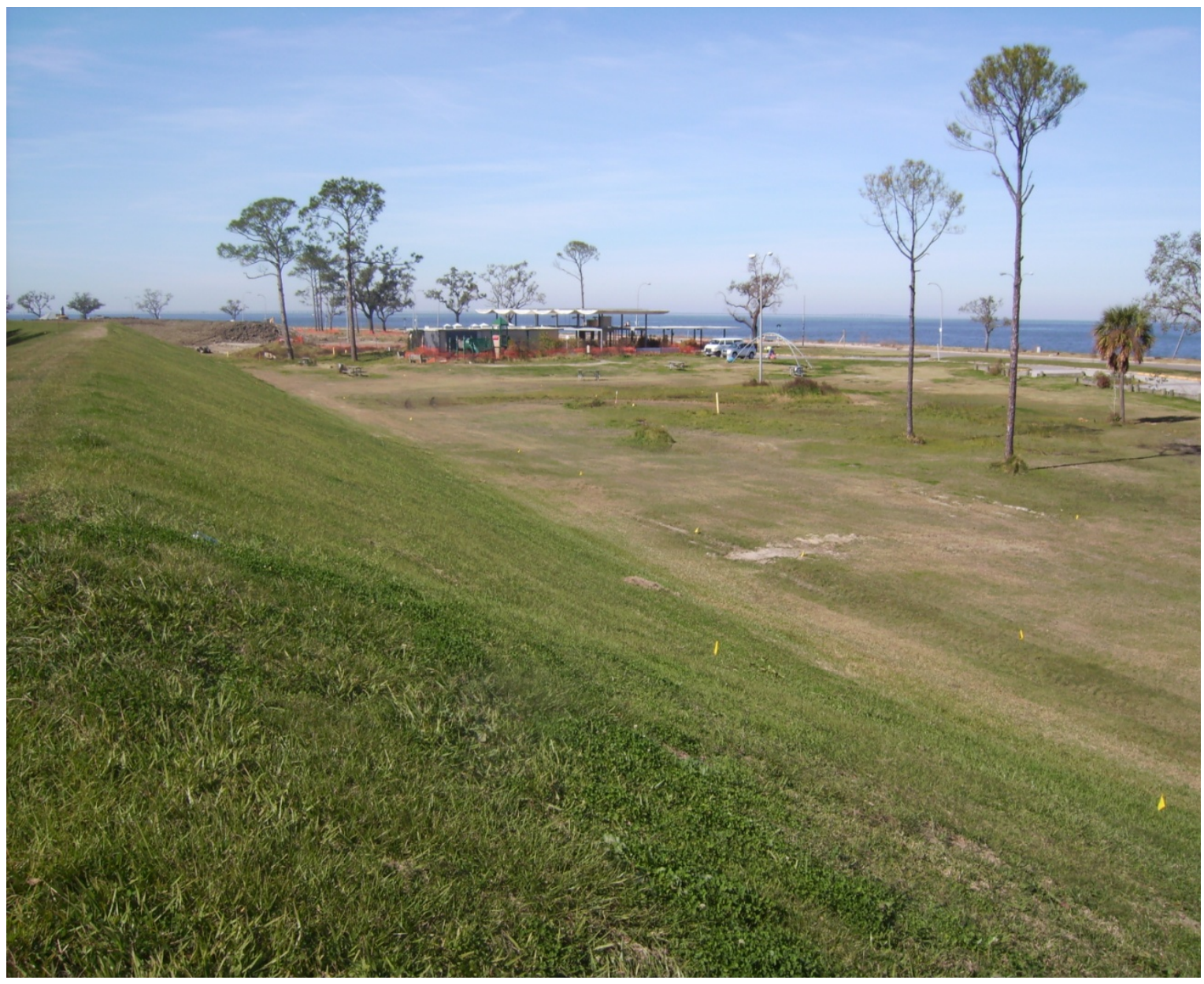


The US Army Engineer Research and Development Center (ERDC) solves the nation's toughest engineering and environmental challenges. ERDC develops innovative solutions in civil and military engineering, geospatial sciences, water resources, and environmental sciences for the Army, the Department of Defense, civilian agencies, and our nation's public good. Find out more at www.erdc.usace.army.mil.

To search for other technical reports published by ERDC, visit the ERDC online library at http://acwc.sdp.sirsi.net/client/default. 


\section{Geophysical Surveys for Locating Buried Utilities, Lake Pontchartrain Levees, New Orleans, LA}

José L. Llopis and Janet E. Simms

Geotechnical and Structures Laboratory

US Army Engineer Research and Development Center

3909 Halls Ferry Road

Vicksburg, MS 39180-6199

Grant A. Riddick

US Army Engineer District, Saint Paul

$190 E^{\text {th }}$ Street

Suite 401

Saint Paul, MN 55101

Final report

Approved for public release; distribution is unlimited.

Prepared for US Army Engineer District, New Orleans 7400 Leake Ave.

New Orleans, LA 70118-3651 


\section{Abstract}

This report presents the results of a geophysical study performed to determine the location of buried utilities beneath or in the vicinity of the levees on the south shore of Lake Pontchartrain approximately $8 \mathrm{~km}$ ( 5 miles) north of downtown New Orleans, LA. There was concern that utilities located beneath or buried near the toe of the levees could act as a water conduit during flooding events. If a water-filled utility fails, it is possible that it may cause the levee to fail either by piping material from within the levee or cause slope stability problems. It is also possible that buried utilities can act as potential seepage paths through the levee during high water events. In this case, the buried pipe would not have to "fail" to cause a problem. The utilities needed to be accurately located so that they could be rerouted, removed, or abandoned and grouted-in. Electromagnetic, total field magnetic, and ground penetrating radar systems were assessed to determine the best method for detecting the buried utilities in this area. The Geonics EM31 electromagnetic induction instrument was considered the most effective for detecting the utilities. EM31 anomalies, presumed to be the locations of buried utilities, were mapped and their coordinates tabulated for further interrogation.

DISCLAIMER: The contents of this report are not to be used for advertising, publication, or promotional purposes. Citation of trade names does not constitute an official endorsement or approval of the use of such commercial products. All product names and trademarks cited are the property of their respective owners. The findings of this report are not to be construed as an official Department of the Army position unless so designated by other authorized documents. 


\section{Contents}

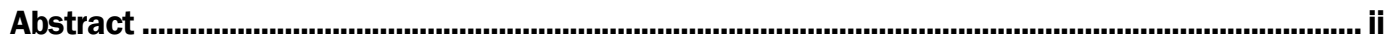

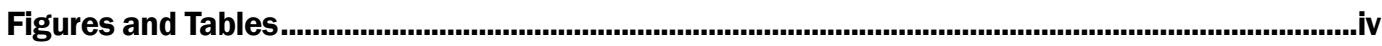

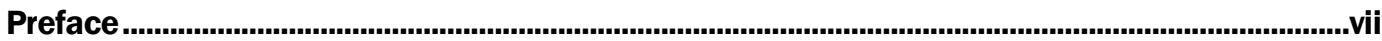

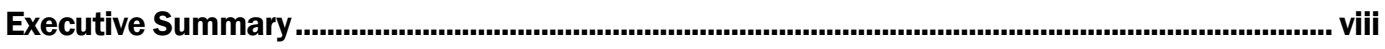

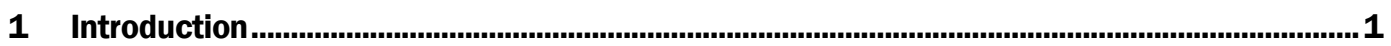

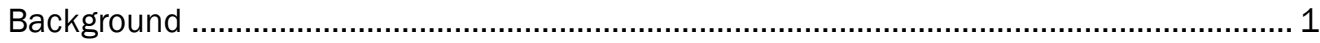

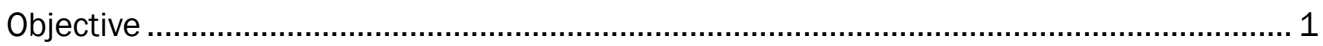

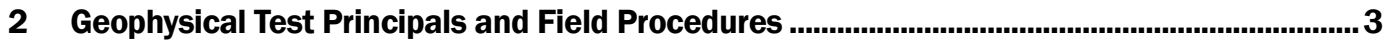

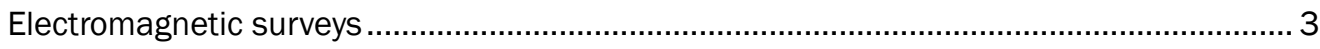

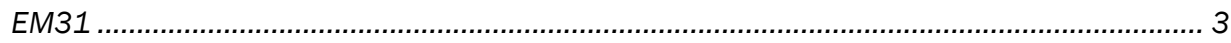

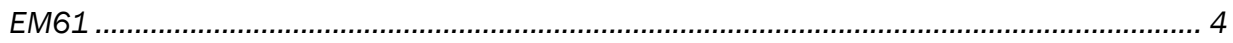

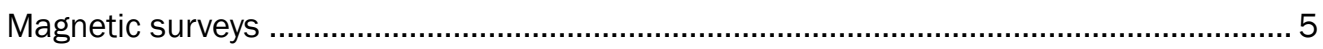

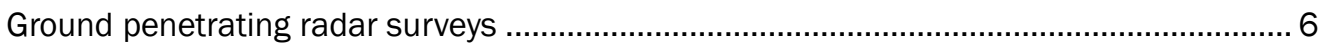

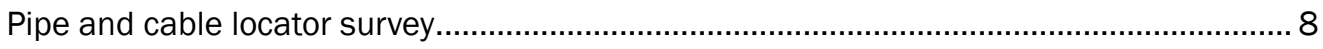

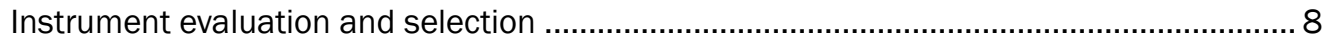

3 Geophysical Test Results.................................................................................................10

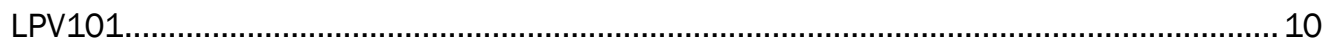

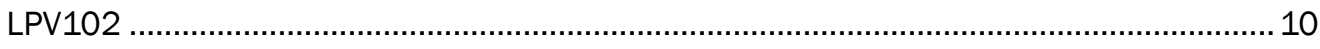

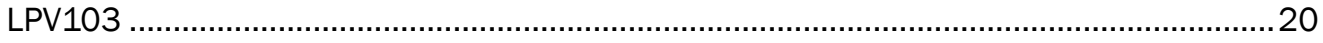

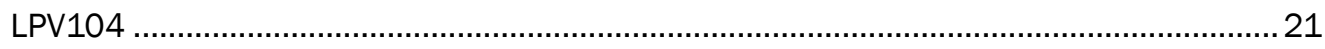

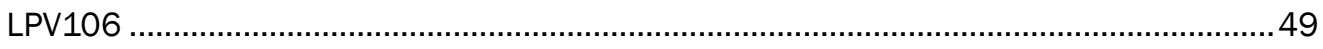

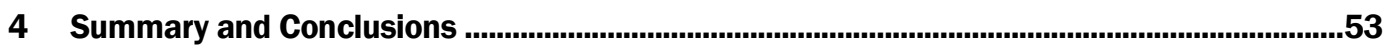

Appendix A: Tabulated Coordinates of Flagged Anomalies and Utility Locations,

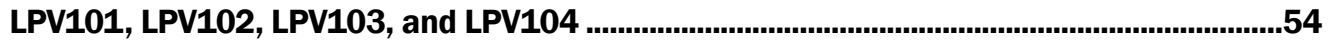

Appendix B: EM31 Conductivity and In-phase Results, LPV101, LPV102, LPV103, and LPV104.

\section{Report Documentation Page}




\section{Figures and Tables}

\section{Figures}

Figure 1. Site map.

Figure 2. Geonics Ltd. EM31 conductivity meter being used during a typical survey........................... 4

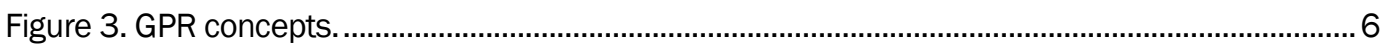

Figure 4. Sensors and Software Noggin Plus Smart Cart system with $250 \mathrm{MHz}$ antenna.................. 7

Figure 5. Illustration of the Radiodetection RD4000 cable and pipe locator system. ........................ 8

Figure 6. LPV101 survey area. ................................................................................................11

Figure 7. Anomalies interpreted from EM31 survey, LPV101_A. ………………………...................12

Figure 8. Anomalies interpreted from EM31 survey, LPV101_B. ....................................................13

Figure 9. Anomalies interpreted from EM31 survey, LPV101_C.........................................................14

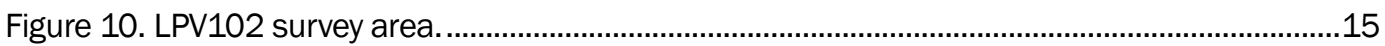

Figure 11. Anomalies interpreted from EM31 survey, LPV102_A .....................................................16

Figure 12. Anomalies interpreted from EM31 survey, LPV102_B.....................................................17

Figure 13. Anomalies interpreted from EM31 survey, LPV102_C..................................................18

Figure 14. Anomalies interpreted from EM31 survey, LPV102_D...................................................19

Figure 15. Anomalies interpreted from EM31 survey, LPV102_E. ..................................................20

Figure 16. LPV103 survey area.................................................................................................22

Figure 17. Anomalies interpreted from EM31 survey, LPV103_A.A. …………………………….....23

Figure 18. Anomalies interpreted from EM31 survey, LPV103_A ........................................................2

Figure 19. Anomalies interpreted from EM31 survey, LPV103_B......................................................25

Figure 20. Anomalies interpreted from EM31 survey, LPV103_C.....................................................26

Figure 21. Anomalies interpreted from EM31 survey, LPV103_D....................................................2

Figure 22. Anomalies interpreted from EM31 survey, LPV103_E. ………………………………...28

Figure 23. Anomalies interpreted from EM31 survey, LPV103_F....................................................2

Figure 24. Anomalies interpreted from EM31 survey, LPV103_G..................................................30

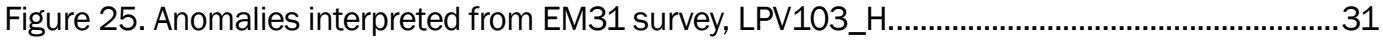

Figure 26. Anomalies interpreted from EM31 survey, LPV103_I. ......................................................32

Figure 27. Anomalies interpreted from EM31 survey, LPV103_J. ………………….......................33

Figure 28. Anomalies interpreted from EM31 survey, LPV103_K.....................................................34

Figure 29. LPV104 survey area...........................................................................................................35

Figure 30. Anomalies interpreted from EM31 survey, LPV104_A ………………………………...36

Figure 31. Anomalies interpreted from EM31 survey, LPV104_B. ....................................................37

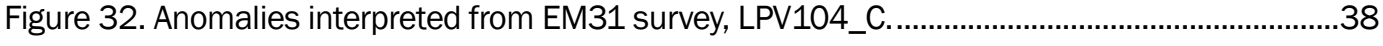

Figure 33. Anomalies interpreted from EM31 survey, LPV104_D.....................................................39

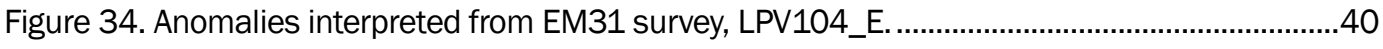

Figure 35. Anomalies interpreted from EM31 survey, LPV104_F........................................................41 
Figure 36. Anomalies interpreted from EM31 survey, LPV104_G...................................................42

Figure 37. Anomalies interpreted from EM31 survey, LPV104_H. ..................................................43

Figure 38. Anomalies interpreted from EM31 survey, LPV104_I. .........................................................4

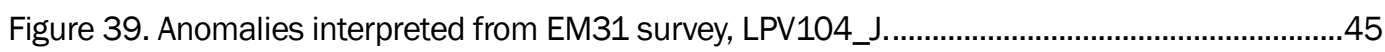

Figure 40. Anomalies interpreted from EM31 survey, LPV104_K.................................................46

Figure 41. Excavating an EM31 anomaly marked with yellow flags, LPV104_K. UTM Coordinate $(785579.6,3325961.8)$.......................................................................................... 47

Figure 42. Cable located with the EM31 at bottom of excavation, LPV104_K. UTM

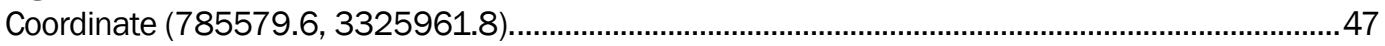

Figure 43. Radio transmitter placed in storm sewer, UTM coordinates (785524.6, 3325885.6), LPV104_K.

Figure 44. Radio transmitter in storm sewer being tracked on the ground surface. Yellow flags indicate storm sewer track. 49

Figure 45. Location of the six seepage areas investigated along Hayne Boulevard, LPV106. .50

Figure 46. Seepage area, indicated by the white flag on the toe of the levee, located near the intersection of Hayne Boulevard and Pebble Drive. .51

Figure 47. Key for Table 2. 51

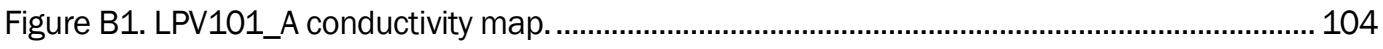

Figure B2. LPV101_A EM31 in-phase map. ............................................................................. 105

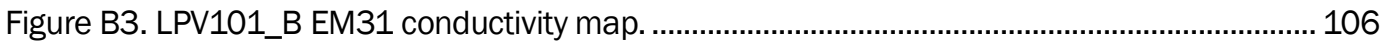

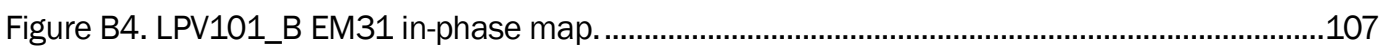

Figure B5. LPV101_C EM31 conductivity map. …………....................................................... 108

Figure B6. LPV101_C EM31 in-phase map. ............................................................................. 109

Figure B7. LPV102_A EM31 conductivity map...................................................................... 110

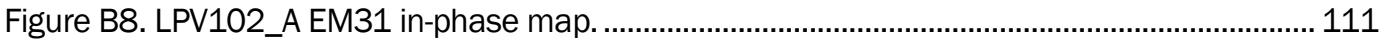

Figure B9. LPV102_B EM31 conductivity map. .................................................................. 112

Figure B10. LPV102_B EM31 in-phase map. ……………........................................................ 113

Figure B11. LPV102_C EM31 conductivity map..................................................................... 114

Figure B12. LPV102_C EM31 in-phase map. ………………………………………….... 115

Figure B13. LPV102_D EM31 conductivity map. ………….................................................. 116

Figure B14. LPV102_D EM31 in-phase map. ..............................................................................117

Figure B15. LPV102_E EM31 conductivity map ..................................................................... 118

Figure B16. LPV102_E EM31 in-phase map...................................................................... 119

Figure B17. LPV103_AA EM31 conductivity map. ……………................................................ 120

Figure B18. LPV103_AA EM31 in-phase map. …………………………………………….... 121

Figure B19. LPV103_A EM31 conductivity map........................................................................ 122

Figure B20. LPV103_A EM31 in-phase map............................................................................. 123

Figure B21. LPV103_B EM31 conductivity map....................................................................... 124

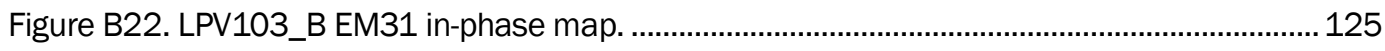

Figure B23. LPV103_C EM31 conductivity map.....................................................................126

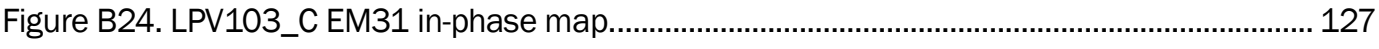

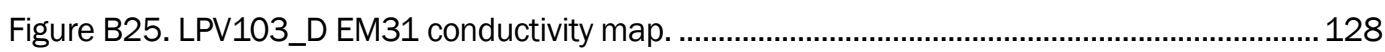




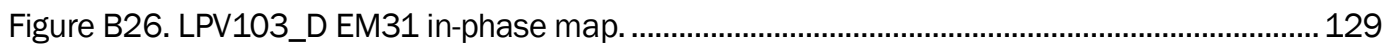

Figure B27. LPV103_E EM31 conductivity map. ................................................................... 130

Figure B28. LPV103_E EM31 in-phase map......................................................................... 131

Figure B29. LPV103_F EM31 conductivity map.................................................................... 132

Figure B30. LPV103_F EM31 in-phase map........................................................................ 133

Figure 31. LPV103_G EM31 conductivity map. ..................................................................... 134

Figure B32. LPV103_G EM31 in-phase map. ........................................................................... 135

Figure B33. LPV103_H EM31 conductivity map. …………………………………............... 136

Figure B34. LPV103_H EM31 in-phase map. …………….................................................. 137

Figure B35. LPV103_I EM31 conductivity map....................................................................... 138

Figure B36. LPV103_I EM31 in-phase map............................................................................ 139

Figure B37. LPV103_J EM31 conductivity map....................................................................... 140

Figure B38. LPV103_J EM31 in-phase map. ............................................................................141

Figure B39. LPV103_K and LPV104_A EM31 conductivity map. ................................................... 142

Figure B 40. LPV103_K and LPV104_A EM31 in-phase map..................................................... 143

Figure B41. LPV104_B EM31 conductivity map....................................................................... 144

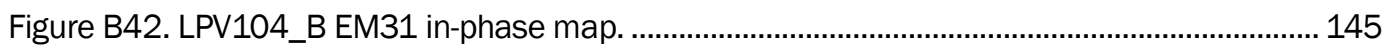

Figure B43. LPV104_C EM31 conductivity map...................................................................... 146

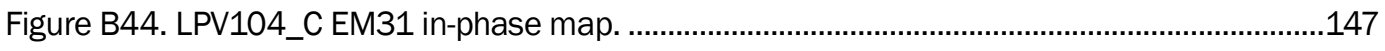

Figure B45. LPV104_D EM31 conductivity map. …………………………………................. 148

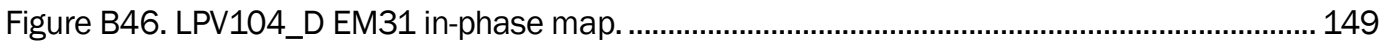

Figure B47. LPV104_E EM31 conductivity map........................................................................ 150

Figure B48. LPV104_E EM31 in-phase map....................................................................... 151

Figure B49. LPV104_F EM31 conductivity map ...................................................................... 152

Figure B50. LPV104_F EM31 in-phase map............................................................................ 153

Figure B51. LPV104_G EM31 conductivity map...................................................................... 154

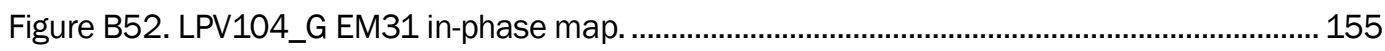

Figure B53. LPV104_H EM31 conductivity map. …………………………………………..... 156

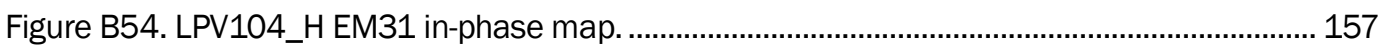

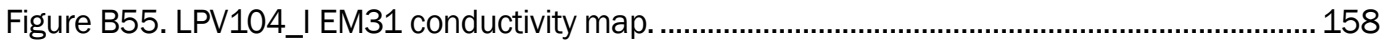

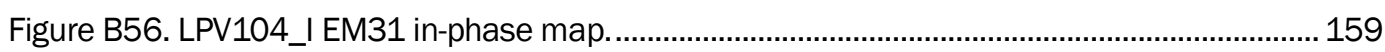

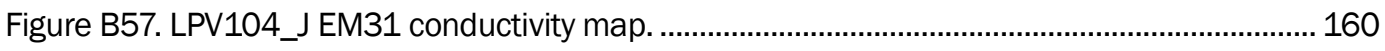

Figure B58. LPV104_J EM31 in-phase map. ……………...................................................... 161

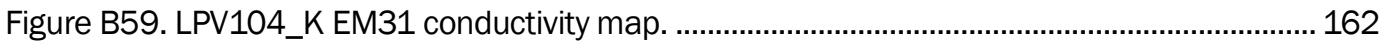

Figure B60. LPV104_K EM31 in-phase map. ........................................................................... 162

\section{Tables}

Table 1. Coordinates of seepage locations along Hayne Boulevard, LPV-106. .52

Table 2. Seepage locations along Hayne Boulevard, LPV-106, referenced to nearest expansion joints and road curb. 


\section{Preface}

This report describes a study commissioned by the US Army Engineer District, New Orleans, under Military Interdepartmental Purchase Request W42HEM73138891 and conducted by the US Army Engineer Research and Development Center (ERDC) to map buried utilities beneath and near the levees on the south shore of Lake Pontchartrain, New Orleans, LA, using surface-based geophysical methods. The work was performed during the periods 29 November through 7 December 2007, 9 through 18 J anuary 2008, 22 J anuary through 1 February 2008, 19 through 24 February 2008, and 15 through 16 April 2008. The geophysical surveys included electromagnetic induction, ground penetrating radar, total field magnetics, and radio signal detection.

The Senior Project Manager, Hurricane Protection Office (HPO), New Orleans District, was Kevin G. Wagner. The work described herein was conducted by J osé L. Llopis and Dr. J anet E. Simms, Geotechnical Engineering and Geosciences Branch (GEGB), Geosciences and Structures Division (GSD), Geotechnical and Structures Laboratory (GSL), ERDC, and Grant A. Riddick, US Army Engineer District, Saint Paul. This publication was prepared by Llopis under the general supervision of Chad A. Gartrell, Chief, GEGB; Bartley P. Durst, Chief, GSD; Dr. William P. Grogan, Deputy Director, GSL; and Dr. David W. Pittman, Director, GSL. The report was published under the Flood and Coastal Storm Damage Reduction Program.

COLJ effrey R. Eckstein was Commander of ERDC. Dr. J effery P. Holland was Director. 


\section{Executive Summary}

Substantial portions of the hurricane protection system restoration effort in New Orleans, LA, are being undertaken by the upper five districts (U5) in the US Army Corps of Engineers Mississippi Valley Division. This work was requested initially by the U5 project manager William L. Csajko, US Army Engineer District, Saint Paul, for the levee reach designated as Lake Pontchartrain and Vicinity (LPV) 103 and funded through the Hurricane Protection Office (HPO), Senior Project Manager, Kevin G. Wagner, US Army Engineer District, New Orleans.

Ultimately, the work expanded to include additional levee reaches designated as LPV101, LPV102, and LPV104 and a partial portion of LPV106. These areas are all located along the south shore of Lake Pontchartrain in neighborhoods known locally as Lakeview (LPV101-104) and New Orleans East (LPV106).

Subsurface geophysical methods can be broadly characterized as an attempt to "see" beneath the ground surface in a non-destructive and non-intrusive manner (without digging up the ground). In any area, the ground soils have naturally occurring physical properties associated with them. The measurement of these naturally occurring properties may be considered the background readings or "normal" readings for a given area. In reality, what the geophysical instruments detect are significant changes (anomalies) in one of these naturally occurring physical parameters. One of the primary tasks of the geophysical professional is to select the most effective geophysical method for the site given its native conditions. It is important to emphasize that while various geophysical methods are available, not all are applicable in all ground conditions. The limitations of the various methods employed during this project are discussed in detail within the main body of this report. The most effective and efficient method for the conditions encountered along the Pontchartrain levee alignments, as determined by the authors in the field, was the electromagnetic (EM) survey method.

The general process employed for field data collection was to traverse the ground adjacent to the levee as well as the levee prism itself with a Geonics EM31 terrain conductivity meter. The EM method "sees" anomalies most effectively when in a perpendicular orientation to the anomaly. By crossing 
the area in both a "north-south" and "east-west" orientation, it was hoped that anomalies both perpendicular and parallel to the levees would be detected. Detected anomalies were field marked with pin flags, and their locations later recorded using a global positioning system (GPS). Anomalous locations were mapped and linear trending anomalies were interpreted to be potential buried utility lines. The final determination of the actual nature and true depth of these linearly trending anomalies is only possible through excavation and visible inspection. The use of geophysical techniques for utility detection enables the extent of subsequent excavating to be minimized by more accurately targeting a potential utility location. 


\section{Introduction}

\section{Background}

Levees in urban areas often times are located over or near buried utilities. During flooding events, it is conceivable that utilities encased in conduits crossing beneath levees or buried near levee toes may become in-filled with water from flood waters on the flood side of the levee. If one of these waterfilled conduits were to fail beneath or near the levee toe during a flood event, a piping-induced catastrophic failure of the levee is possible. It is also possible that buried utilities can serve as potential seepage paths through the levee during high-water events. In this case, the buried pipe would not have to "fail" to cause a problem. Currently, the US Army Engineer District, New Orleans (MVN), Hurricane Protection Office (HPO), is enlarging levees in an urban area located on the south shore of Lake Pontchartrain approximately $8 \mathrm{~km}$ (5 miles) north of downtown New Orleans, LA. Prior to levee enlarging, the HPO located buried utilities near the Lake Pontchartrain levees based on utility maps provided by local utility companies. However, given the length of time the levees have been in place and the urban expansion into this area, the accuracy and completeness of these utility maps are in doubt. These utilities need to be accurately located so that they can be rerouted or abandoned. If abandoned, they must be removed or grouted-in such that they will not let water pass through.

\section{Objective}

At the request of HPO, personnel of the US Army Engineer Research and Development Center (ERDC) conducted a geophysical investigation along a stretch of levee located on the south shore of Lake Pontchartrain (Figure 1). The main study area is approximately $9 \mathrm{~km}$ ( 5.5 miles) in length and is divided into four survey areas; LPV101, LPV102, LPV103, and LPV104 as shown in Figure 1. The primary objective of the investigation was to map the location of anomalies presumably associated with buried utilities beneath and along the toes of the Lake Pontchartrain levees. A secondary objective was to locate the source of localized seepage at six locations along approximately $2 \mathrm{~km}$ (1.3 miles) of the protected side levee toe on Haynes Boulevard. This area is located east of the main study area and designated LPV106 (Figure 1). The investigation was performed during the time periods 29 November through 7 December 2007, 9 through 18 J anuary 2008, 22 J anuary through 1 February 2008, 19 through 24 February 2008, and 15 through 16 April 2008. 


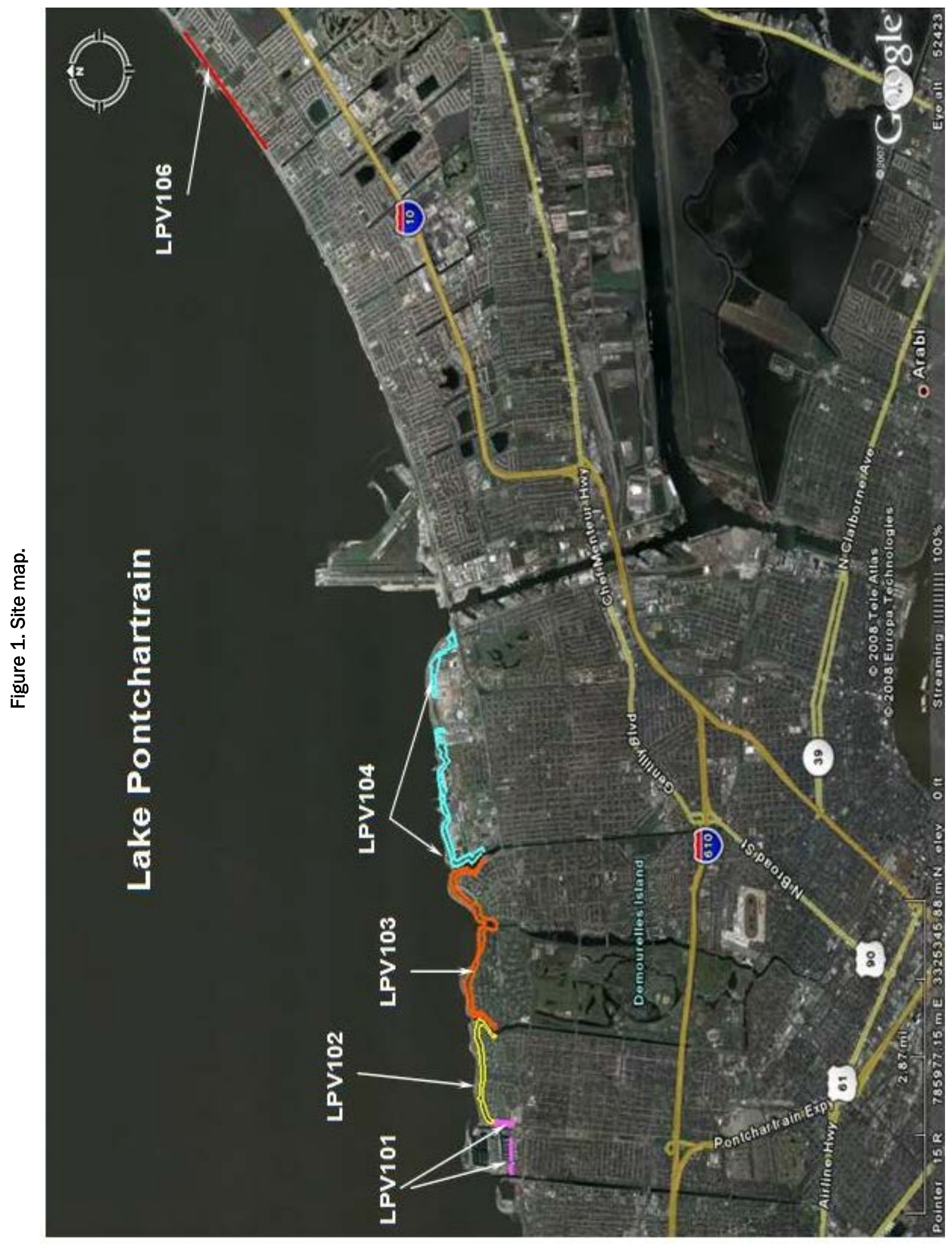




\section{Geophysical Test Principals and Field Procedures}

This section provides a description of the surface geophysical methods and field procedures used for this study. Electromagnetic (EM) induction, magnetic, and ground penetrating radar (GPR) geophysical methods were evaluated to determine which method was best suited for mapping buried utilities. A pipe and cable locator was also used.

\section{Electromagnetic surveys}

EM induction is used to measure the apparent electrical conductivity (inverse of electrical resistivity) of subsurface materials and also for detecting buried metallic items. Electrical conductivity is a measure of the degree to which the soil conducts an electrical current and can be used to infer geologic materials and the location of the water table.

Geonics, Ltd. electromagnetic instruments, models EM31 and EM61 MkII, were used for this study. The EM31 operates in the frequency domain, whereas the EM61 operates in the time domain.

\section{EM31}

The EM31 consists of a set of co-planar transmitter ( $\mathrm{Tx})$ and receiver $(\mathrm{Rx})$ coils separated approximately $3.67 \mathrm{~m}$ (12 ft) apart. An alternating current is passed through the Tx coil, thus generating a primary time varying magnetic field. This primary field induces eddy currents in subsurface conductive materials. The induced eddy currents are the source of a secondary magnetic field, which is detected by the Rx coil along with the primary field.

Two components of the induced magnetic field are measured by the EM system. The first is the quadrature phase, sometimes referred to as the outof-phase or imaginary component. Apparent ground terrain conductivity is determined from the quadrature component. Disturbances in the subsurface caused by compaction, in-filled abandoned channels, soil removal and fill activities, buried objects, or voids may produce conductivity readings different from background values, thus indicating anomalous areas. The units of apparent ground conductivity are measured in milliSiemens per meter $(\mathrm{mS} / \mathrm{m})$. The inphase component is sensitive to 
metallic objects and therefore is useful when looking for buried metal such as metal pipes and electrical wires. When measuring the inphase component, the true zero level is not known, since the referencelevel is arbitrarily set by the operator. Therefore, measurements collected in this mode are relative to an arbitrary reference level and have units of parts per thousand (ppt).

Under optimal conditions, the EM31 has an effective depth of investigation

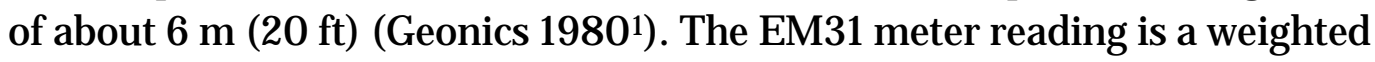
average of the earth's conductivity. A thorough investigation to a depth of

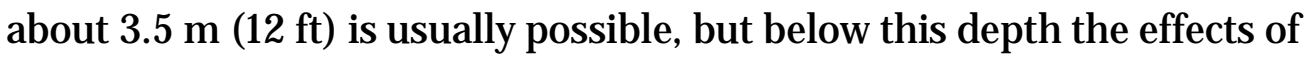
electrically conductive objects become more difficult to distinguish. For this study, the EM31 was carried at hip level, approximately $1 \mathrm{~m}$ ( $3 \mathrm{ft}$ ) above the ground surface, with the long axis of the instrument oriented parallel to the survey line (Figure 2).

Figure 2. Geonics Ltd. EM31 conductivity meter being used during a typical survey.

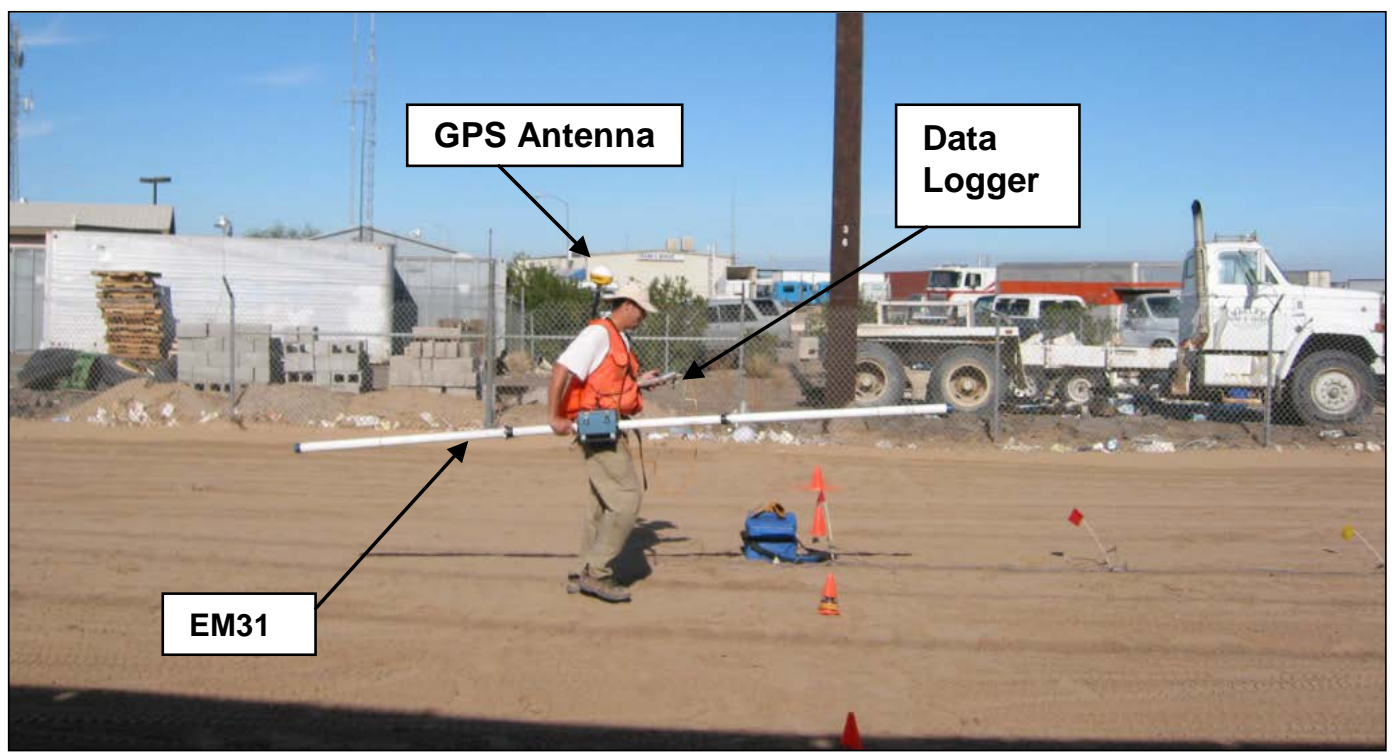

\section{EM61}

The EM61 is a high-resolution, high-sensitivity, time-domain metal detector capable of detecting both ferrous and non-ferrous metallic objects. Unlike the EM31, which applies a continuous alternating current to coil and measures the secondary magnetic field while the transmitter is operating, the EM61 generates a pulsed primary magnetic field, which induces eddy

\footnotetext{
1 Geonics. 1980. Electromagnetic terrain conductivity measurements at low induction numbers. Technical Note TN-6. Mississauga, Ontario, Canada: Geonics Limited..
} 
currents in nearby metallic objects. The decay of these currents is measured by two receiver coils mounted on the coil assembly. To eliminate the effects of conductive soils, which have a shorter decay rate than those of metals, the secondary magnetic field response is not measured until a few microseconds after the transmitter is turned off. The EM61 is capable of detecting a single 55-gal drum at a depth of approximately $3 \mathrm{~m}$ (10 ft) beneath the instrument, yet is relatively insensitive to interference from nearby surface metal such as fences, buildings, cars, etc. (Geonics 20051).

The EM61 consists of two horizontal and parallel coils, each $1.0 \mathrm{~m}$ by $0.5 \mathrm{~m}$ ( $3.3 \mathrm{ft}$ by $1.6 \mathrm{ft}$ ) with the lower coil positioned approximately $0.40 \mathrm{~m}$ (16 in.) above the ground and the upper coil approximately $0.5 \mathrm{~m}$ (20 in.) above it. For this survey, the received signal was measured at three time gates, or channels, using the lower coil, and at the third time gate using both coils, allowing a differential measurement. Wheels are attached to the bottom coil so that the instrument can be towed along a survey line. The measured signal is in units of millivolts (mV).

\section{Magnetic surveys}

Magnetic surveys measure changes in the earth's total magnetic field caused by variations in the magnetic mineral content of near-surface rocks and soils or ferrous objects. These variations are generally local in extent. The magnetic response is attributed both to induction by the magnetizing field and to remanent magnetization. Remanent magnetization is permanent magnetization and depends on the thermal and magnetic history of the body; it is independent of the field in which it is measured. Induced magnetization is temporary magnetization that disappears if the material is removed from the inducing field. Generally, the induced magnetization is parallel with and proportional to the inducing field.

Any material or object having a magnetic susceptibility will contribute to the total magnetic field measured by the magnetometer. If an object is present such that its magnetization is great enough to perturb the ambient magnetic field, then it will appear as an anomaly on the magnetic data plot. The size, orientation, depth of burial, magnetic susceptibility, and remanent magnetization of the object determine the magnitude of the anomaly and thus affect the ability of the magnetometer to detect the

\footnotetext{
1 Geonics. 2005. EM61-Mk2 and EM61-Mk2HP 4 channel high sensitivity metal detectors, operating manual. Mississauga, Ontario, Canada: Geonics Limited.
} 
object. For a given susceptibility and remanent magnetization, as the size of the object decreases and/ or depth of burial increases, the magnitude of the anomaly decreases; eventually the anomaly will be undetectable.

A Geometrics G-858 magnetometer was used to collect the magnetic data. The G-858 uses a cesium vapor sensor and measures the total magnetic field (in nanoTesla, nT). The total magnetic field measurement is a summation of the ambient earth field and the induced field resulting from ferrous iron-bearing material within the sensing range of the sensor. Ferrous material can include both man-made and natural sources. Data were collected at 0.1-sec intervals along the survey lines.

\section{Ground penetrating radar surveys}

GPR is a ground-based geophysical instrument that transmits highfrequency EM pulses into the subsurface. The GPR system consists of a transmitting (Tx) and receiving ( $\mathrm{Rx})$ antenna. When the transmitted EM signal impinges upon the boundaries of materials with contrasting electrical properties, some of the EM signal is reflected back to the surface where it is detected by the receiving antenna. The time the signal takes to travel from the transmitting antenna, reflect off a boundary, and be detected by the receiving antenna are amplified, processed, and recorded to provide a "continuous" profile of the subsurface, as illustrated in Figure 3.

Figure 3. GPR concepts.

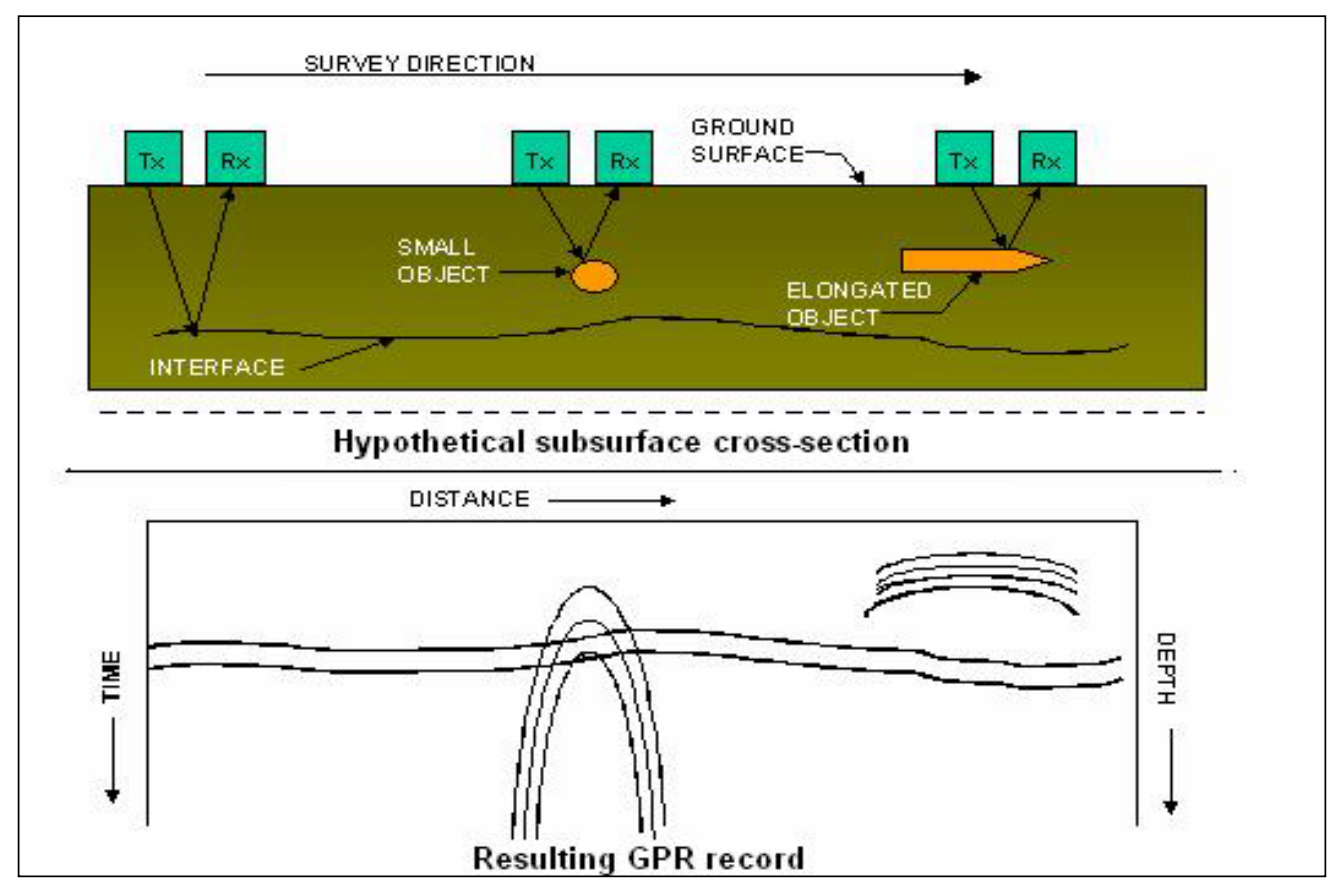


The transmitted EM signals respond to changes in materials with sufficiently different electrical properties such as those caused by mineral content, salinity, water content, density, voids, etc. The depth of penetration and amount of definition that can be expected is determined by the electrical properties of the host material being tested as well as the power and frequency of the transmitting antenna. In general, the higher the conductivity of the host material, the less the GPR depth of penetration will be. The primary disadvantage of GPR is its extremely site-specific applicability. It is difficult to predict whether GPR will be successful in accomplishing its goal without prior knowledge of the electrical properties of the host materials. The GPR system used in this investigation was a Sensors and Software, Inc. Noggin Plus. This GPR system uses a 250-MHz antenna. The reflection profiling survey mode was used for these surveys. In this mode, the receiving and transmitting antennas are kept a fixed distance apart as the antenna pair is pulled along a survey line. The antenna separation for the Noggin Plus 250-MHz antenna is a fixed $35 \mathrm{~cm}$ (14 in.). The cart-mounted Noggin Plus is a user-friendly and straightforward system to operate. The system consists of an antenna and a digital video logger (DVL) all mounted on a cart (Figure 4). Once the unit is unfolded and powered-up, a GPR survey can begin in less than a minute. The DVL is used to input system parameters and collect, display, store, and transfer data.

Figure 4. Sensors and Software Noggin Plus Smart Cart system with $250 \mathrm{MHz}$ antenna.

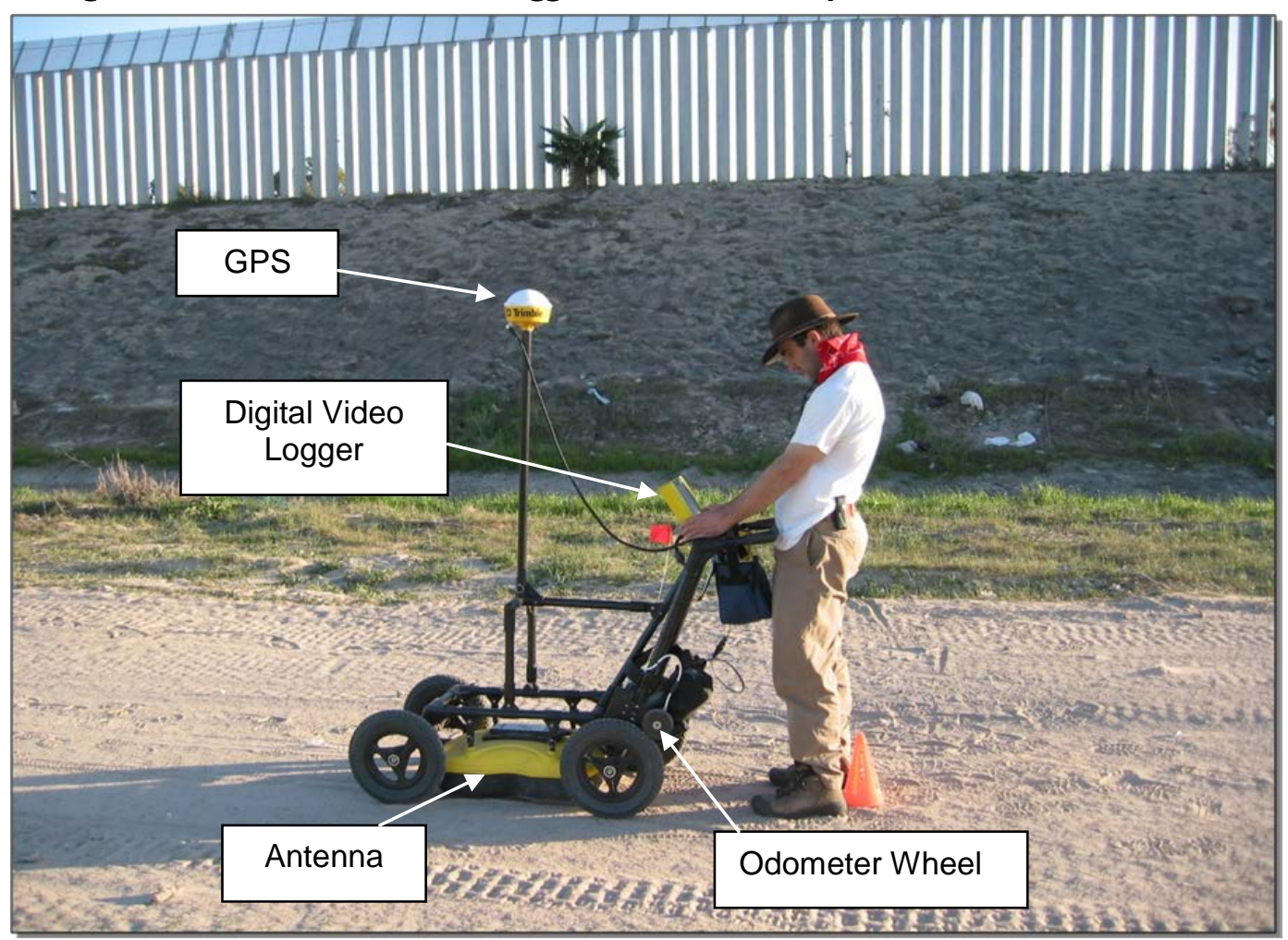




\section{Pipe and cable locator survey}

A Radiodetection RD4000 cable and pipe locator system was used in this study to map the location of a sewer line. The system consists of a radio transmitter attached to the end of a flexible 115-m-long (375-ft) fiberglass rod that is fed into an accessible opening and snaked through the sewer. The system is illustrated in Figure 5. As the transmitter travels through the sewer, the emitted radio signal is detected on the ground surface with the receiver. The transmitter is moved in small increments through the sewer, and its corresponding position is detected and marked on the ground surface. The receiver also displays an estimated depth to the transmitter.

Figure 5. Illustration of the Radiodetection RD4000 cable and pipe locator system.

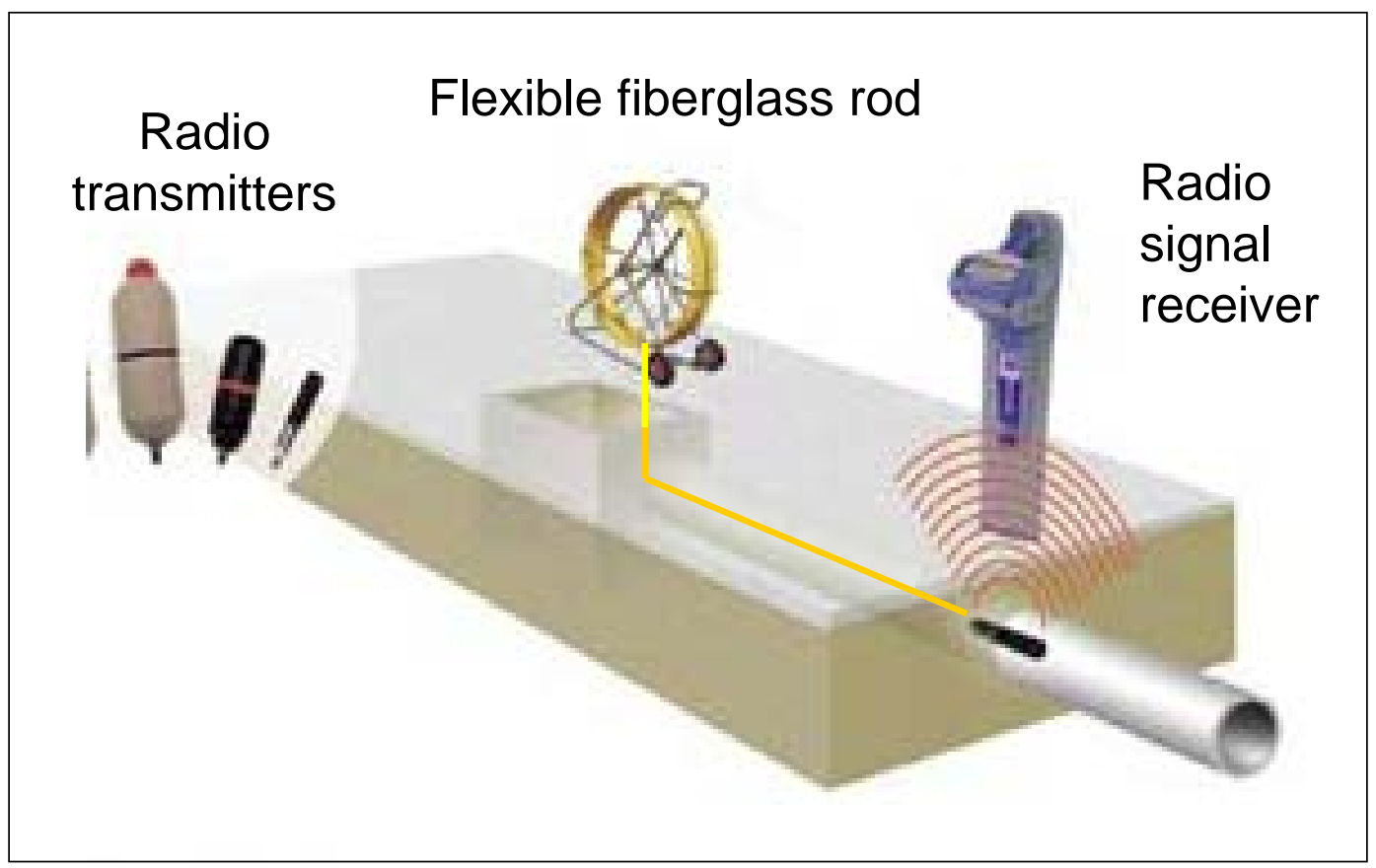

\section{Instrument evaluation and selection}

During the initial field trip, the two EM and the magnetic methods were evaluated to determine which method(s) would be the most effective for mapping utilities on and near the levees. The GPR method was not evaluated at this time, because the levee soils and the soils upon which they are built have a relatively high electrical conductivity making GPR ineffective. GPR surveying was limited to the paved marina area at LPV101 during the $22 \mathrm{~J}$ anuary through 1 February trip. 
The evaluation phase was conducted by laying out several survey lines, each oriented parallel to the levee, along both levee toes and partially up both levee slopes. These survey lines were run to detect buried utilities oriented roughly perpendicular to the levee axis. In addition, lines were laid out in a saw-tooth configuration on both levee toes to detect utilities that ran parallel to or at an oblique angle to the long axis of the levee.

GPR was also evaluated at LPV104 near the intersection of Leon Simon Street, Leroy J ohnson Street, and Lakeshore Drive in an attempt to locate a non-metallic storm sewer line. The GPR was not able to detect the sewer line in this area.

Each instrument was run along the same survey lines. A Trimble DSM 232 global positioning system (GPS) with sub-meter accuracy was used to track and record the locations of the instruments during the surveys. The instrument operator monitored and recorded instrument readings during the survey on a hand-held data logger. Pin flags were placed at the locations where the operator noted an anomaly indicative of a buried metallic object. During the EM61 and magnetometer surveys, many flags that were unrelated to buried utilities were planted because of the instrument's sensitivity to small shallow metallic objects. Ideally, after a survey, the pin flags will line up indicating the location of a buried utility. However, because of the numerous anomalies detected by the EM61 and magnetometer and the planting of corresponding flags, it was difficult to delineate linear features indicative of a buried utility. The EM61 and magnetometer surveys were plotted in plan view and again, because of the interference from the small shallow objects, there was difficulty distinguishing linear features associated with buried utilities.

On the other hand, the EM31 survey results showed far fewer detected anomalies than with the EM61 or the magnetometer. The EM31, being insensitive to shallow small metallic objects, makes the task of distinguishing a buried utility from near-surface objects much easier. Therefore, the EM31 was chosen for locating buried utilities for this investigation. 


\section{Geophysical Test Results}

\section{LPV101}

LPV101 is the westernmost test area of this study area. It is located primarily along Lake Marina Avenue between the east bank of the 17th Street Canal and the intersection of Lakeshore Drive and Topaz Street. It is an urbanized area that has a great deal of metallic clutter that interfered with the EM31. The survey areas within LPV101 are shown in Figure 6. GPR and EM31 surveys were run in the paved parking lot inside the floodwalls by the marina. The EM31 survey was ineffective within the marina site because of the proximity of the reinforced concrete floodwall and parked vehicles, which interfered with the received EM31 signal. Also, the numerous shallow reflectors of varying depth and size detected by the GPR made it impossible to distinguish the clutter from any likely utilities. Figures 7 through 9 show the interpreted anomalies, as indicated by the crosses, from the EM31 surveys conducted at LPV101_A through LPV101_C, respectively. The coordinates of the flagged anomalies are presented in Table A1. The EM31 conductivity and in-phase maps for LPV101 are presented in Figures B1 through B6.

\section{LPV102}

LPV102 is located directly east of LPV101 and extends east along Lakeshore Drive from its intersection with Topaz Street to the west bank of the Orleans Avenue Canal. The site is divided into five sections designated LPV102_A through LPV102_E for presentation purposes (Figure 10). The anomalies for LPV102_A through LPV102_E, based on the results of the EM31 surveys, are presented in Figures 11 through 15, respectively. Survey results for LPV102_A and LPV102_B (Figures 11 and 12, respectively) and LPV102_D (Figure 14) show several linear anomalies crossing perpendicular to the levee. The coordinates of the flagged anomalies for LPV102 are presented in Table A2. The EM31 conductivity and in-phase maps for LPV102 are shown in Figures B7 through B16. 


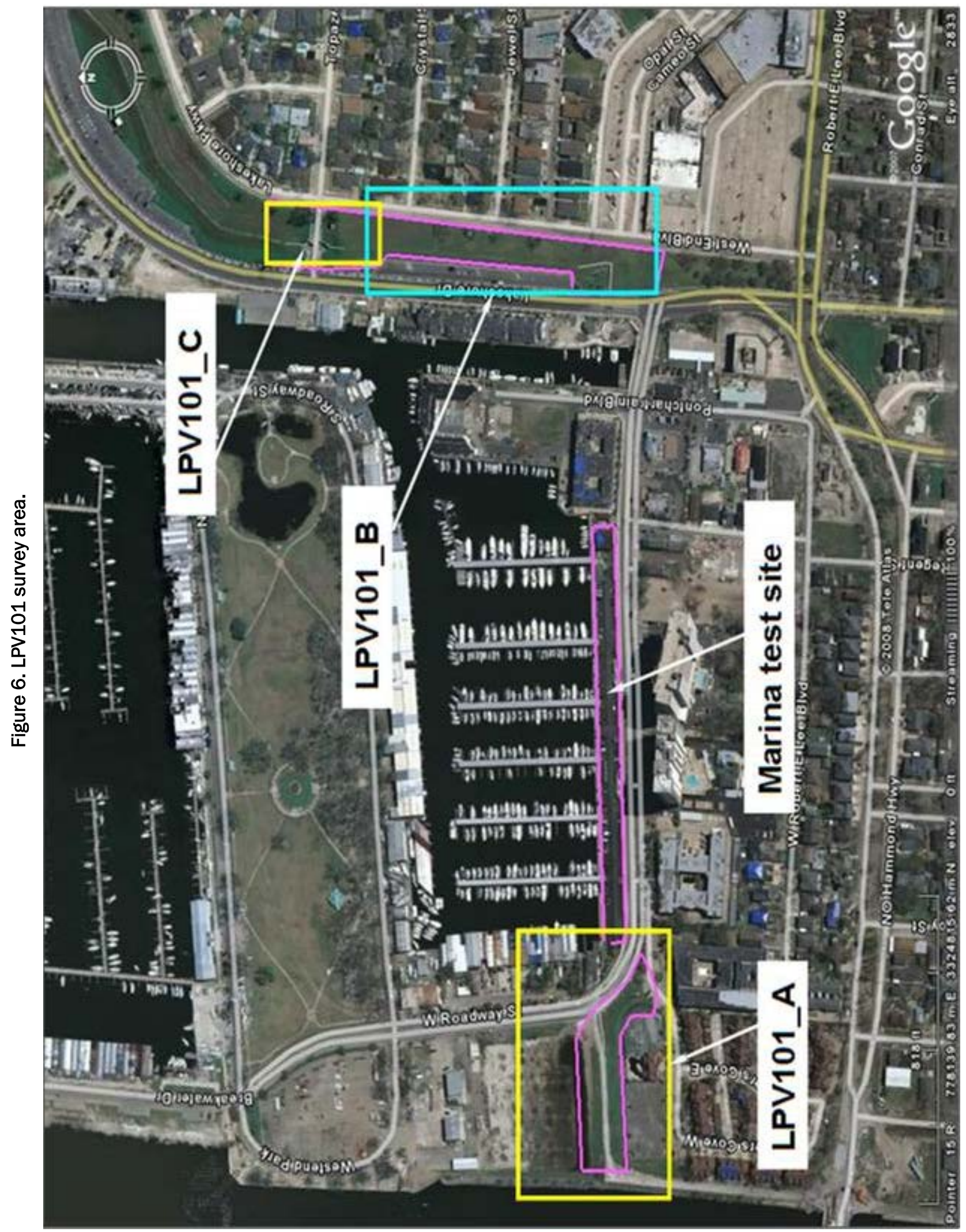




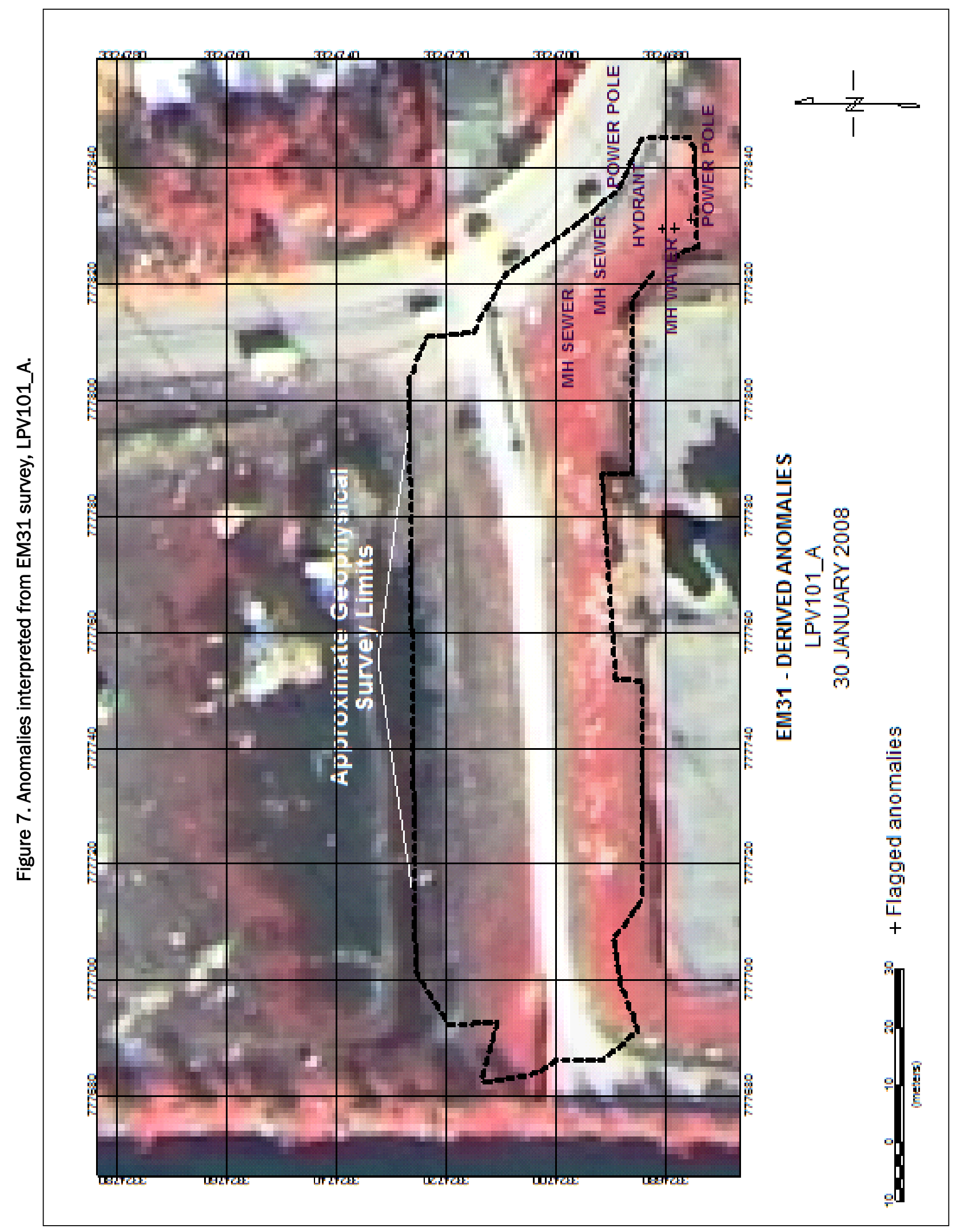


Figure 8. Anomalies interpreted from EM31 survey, LPV101_B.

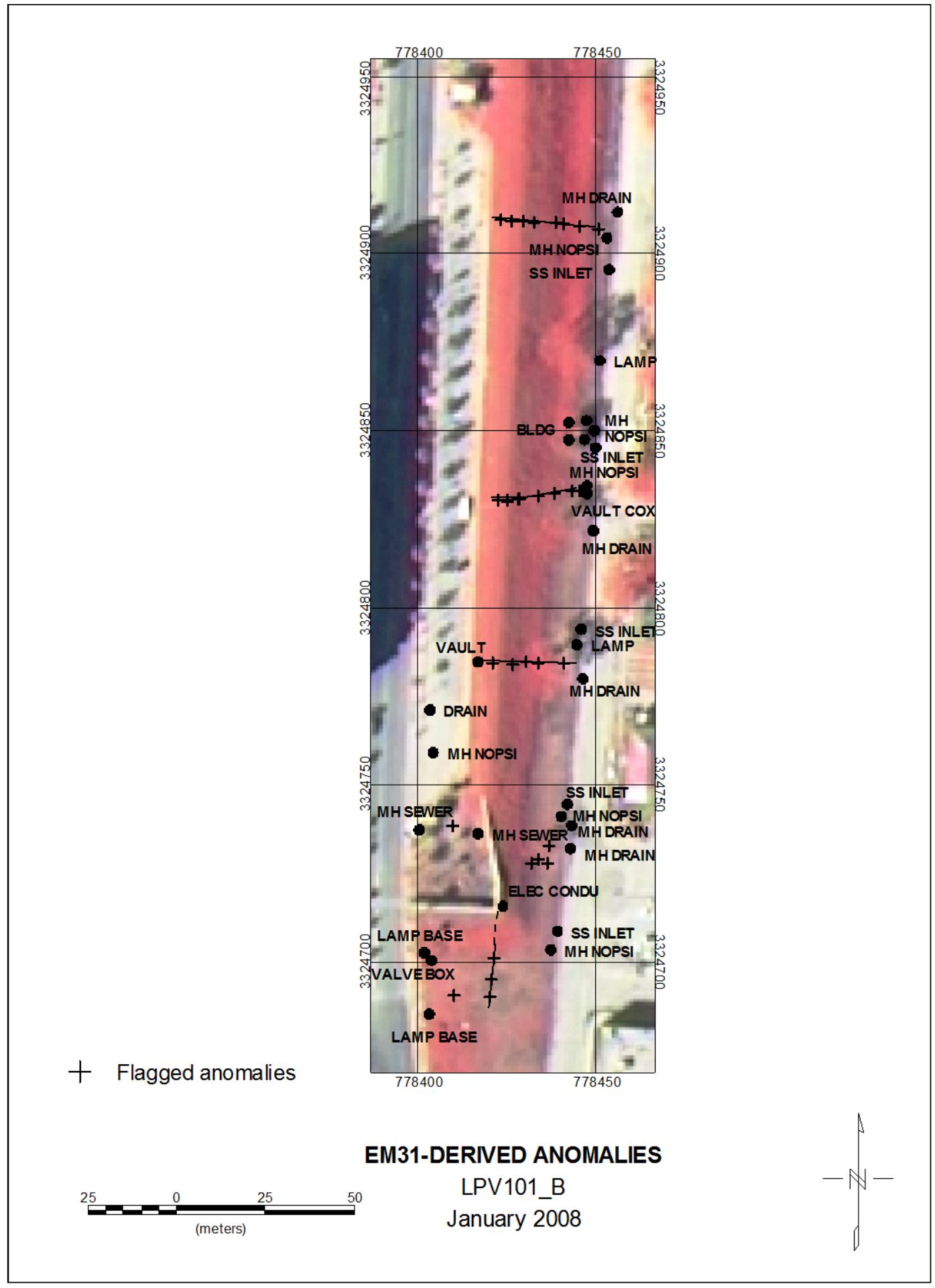


Figure 9. Anomalies interpreted from EM31 survey, LPV101_C.

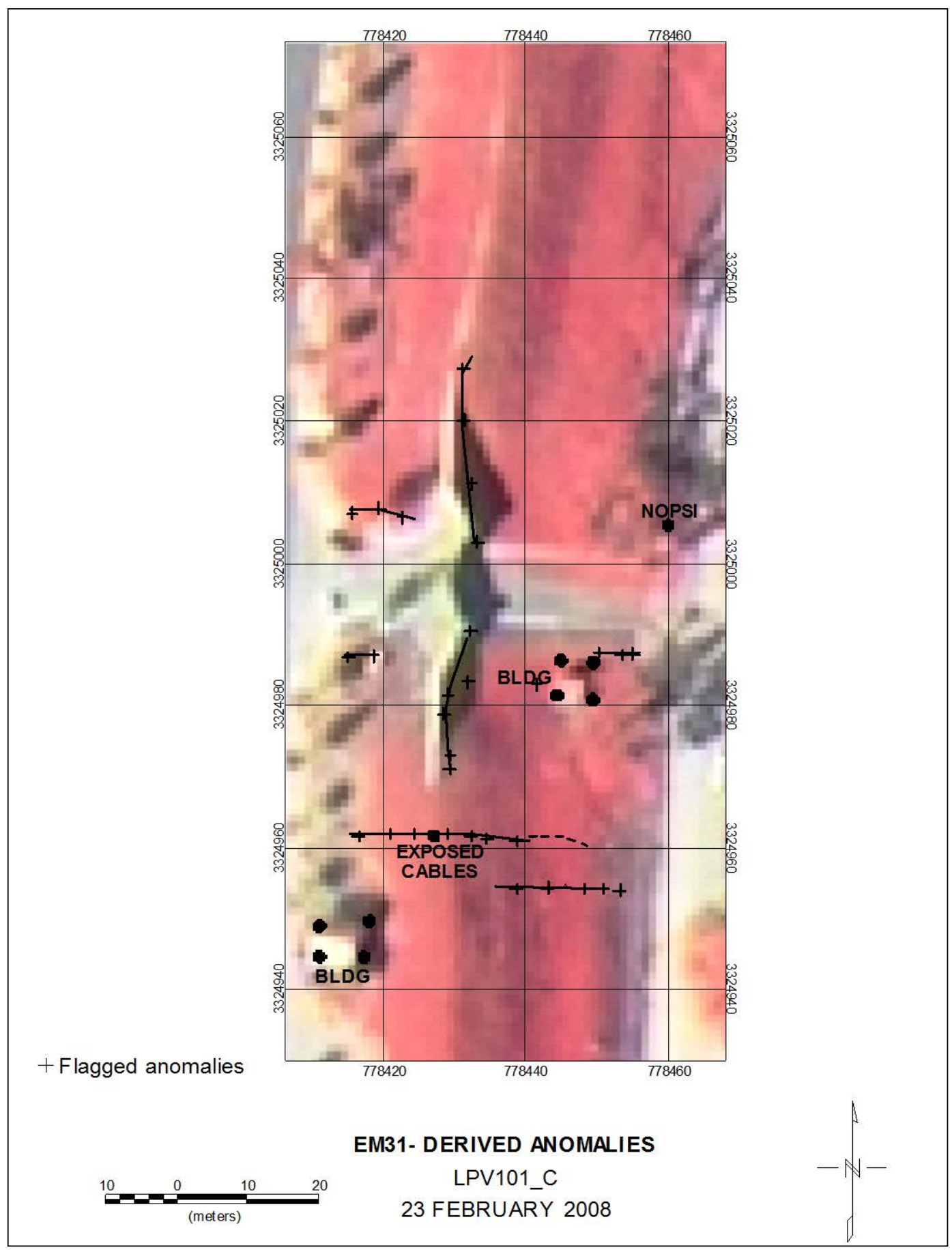




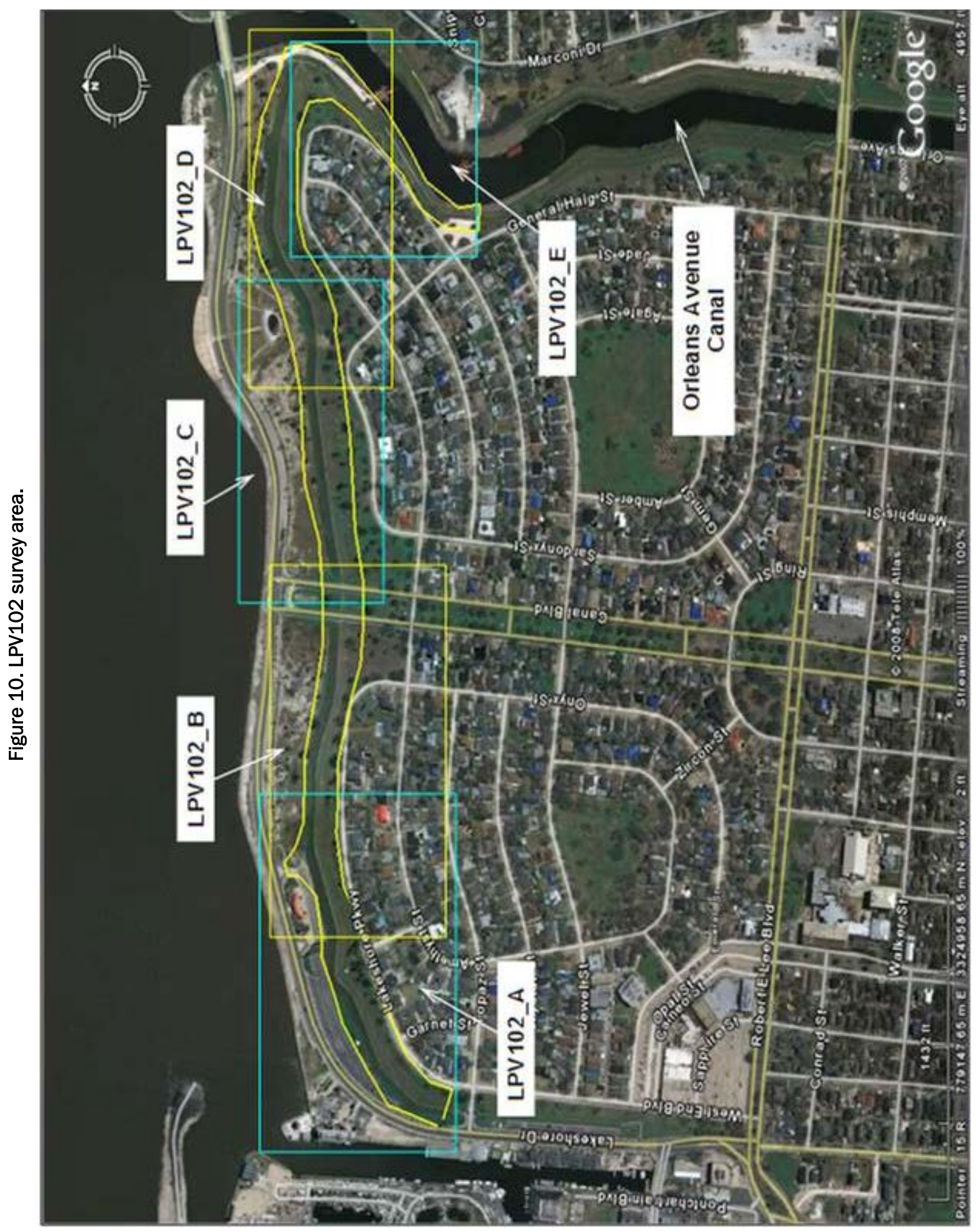




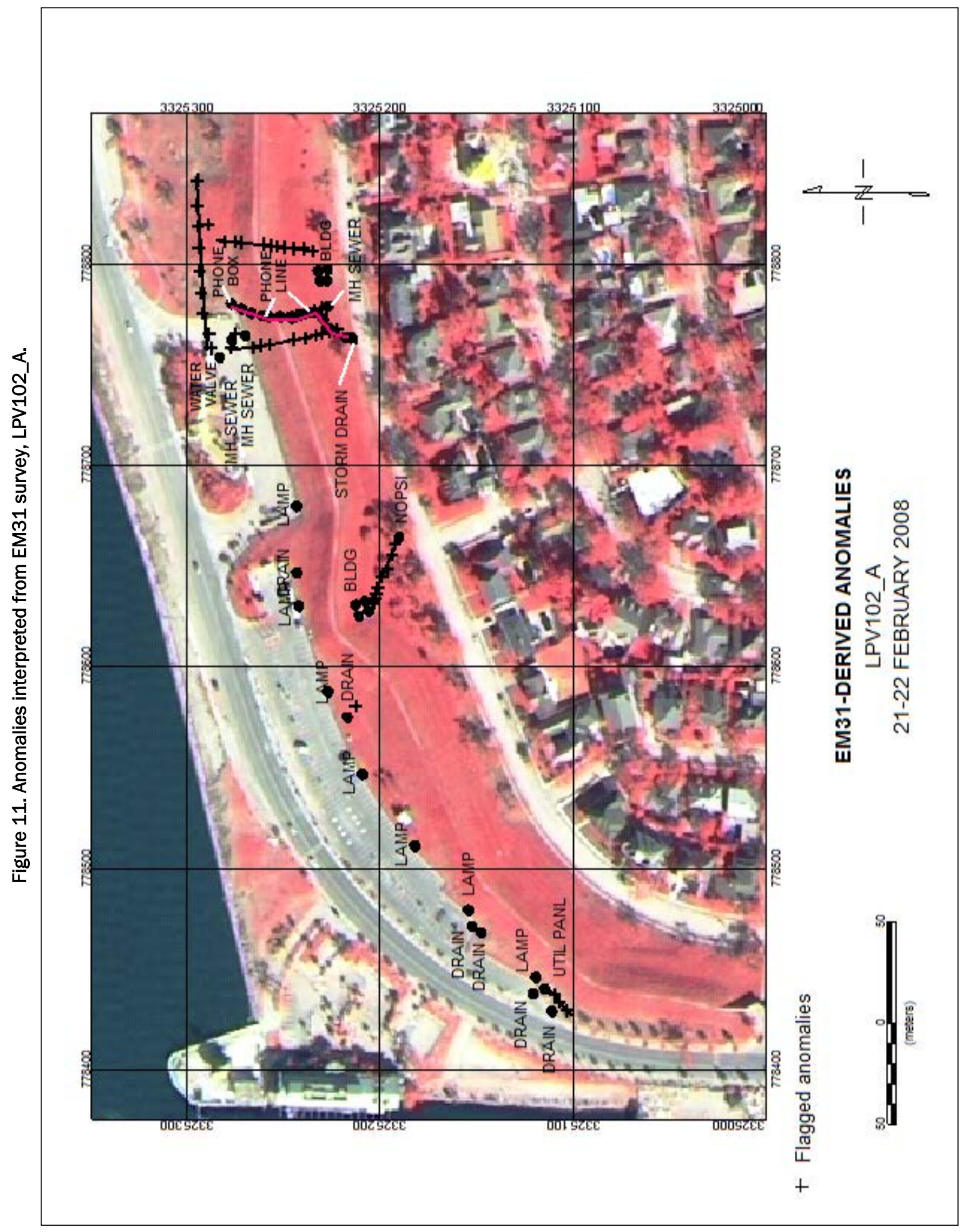




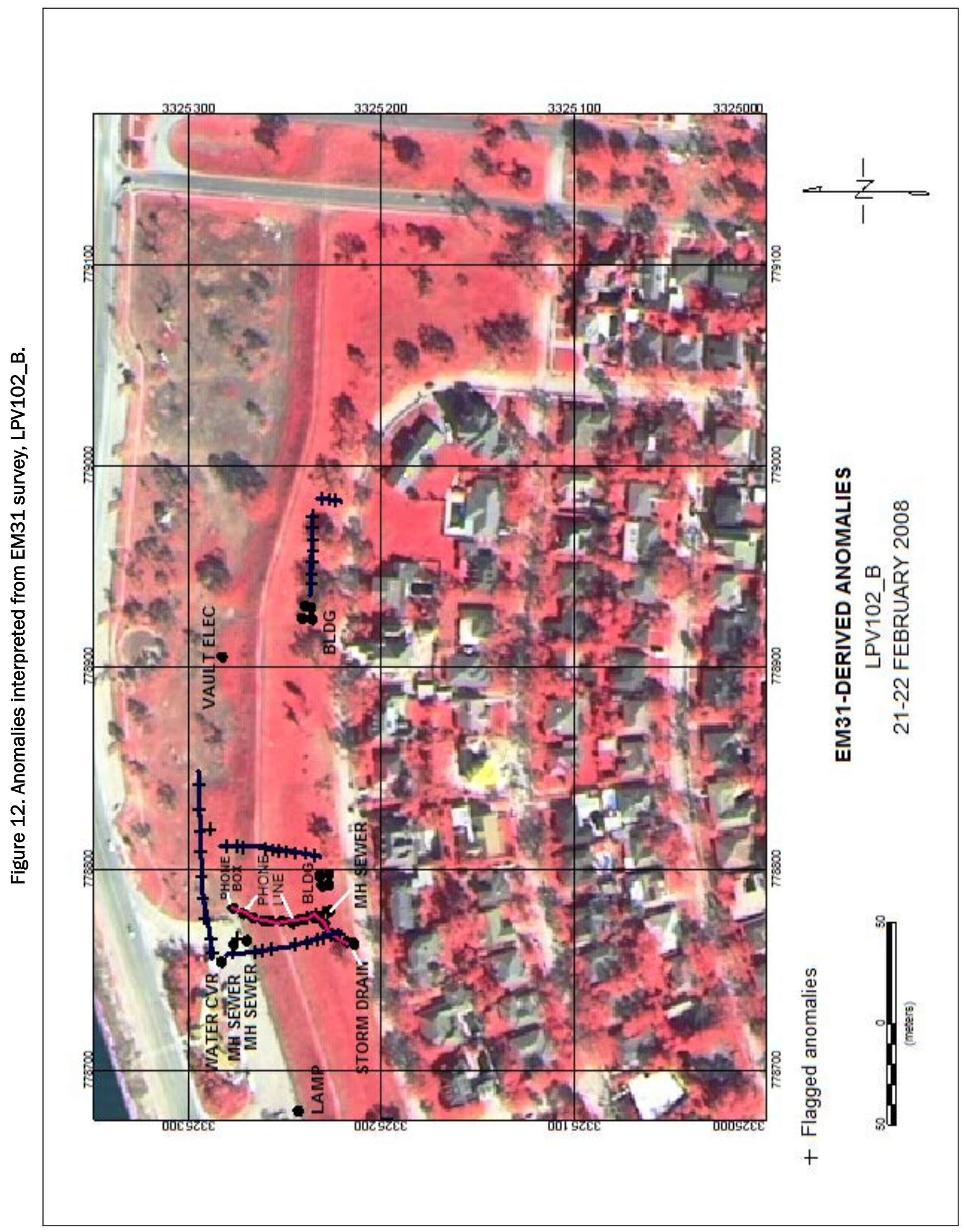




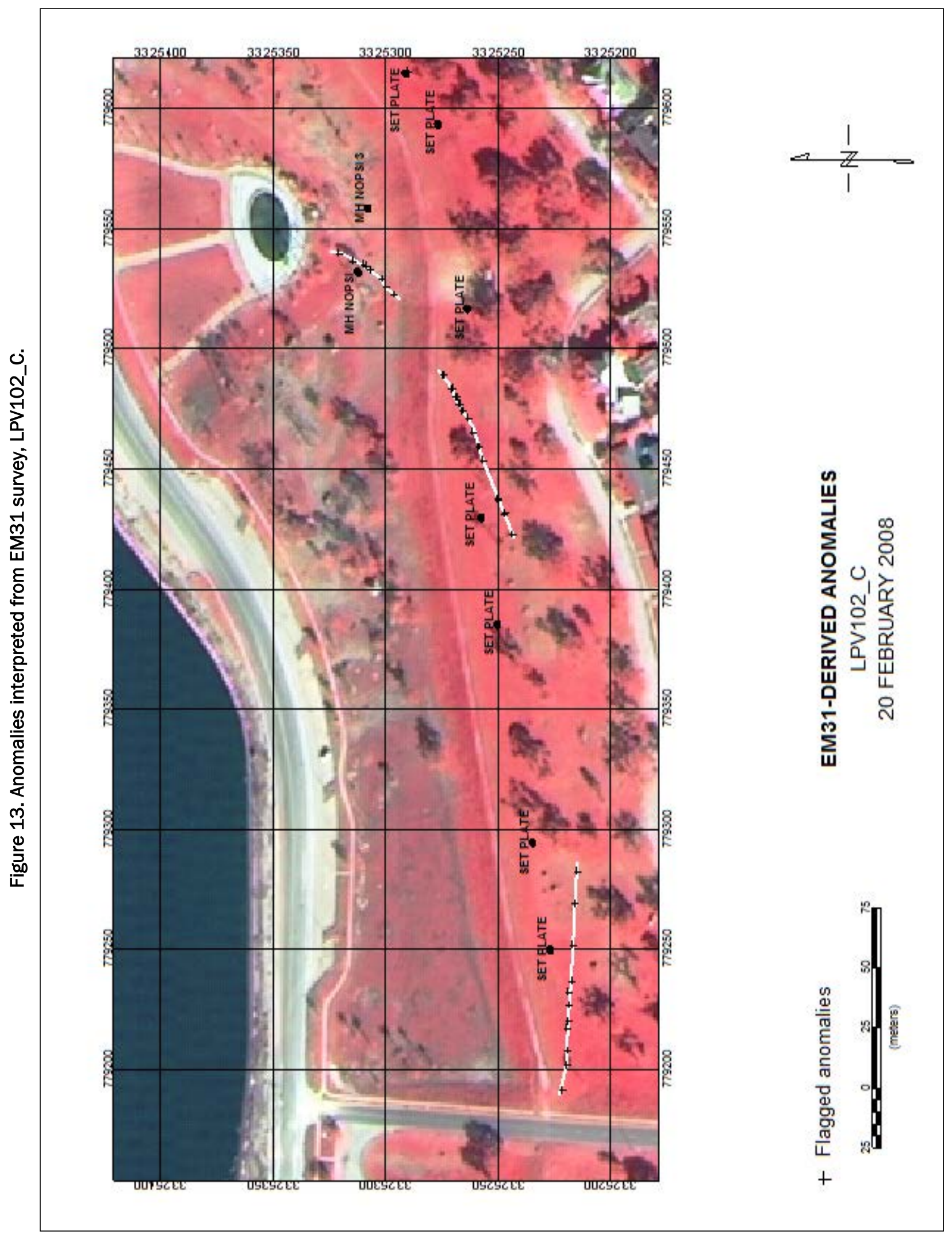




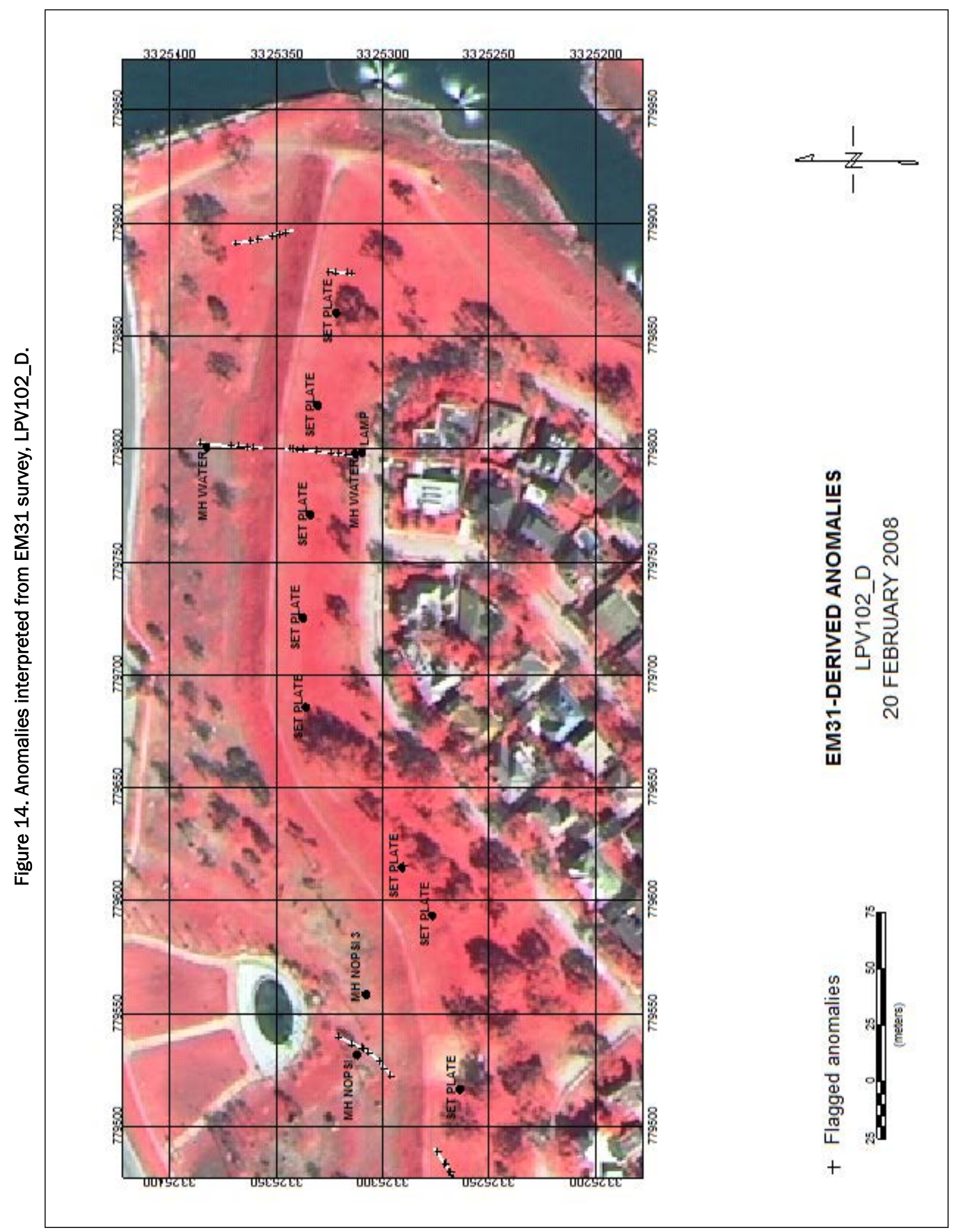


Figure 15. Anomalies interpreted from EM31 survey, LPV102_E.

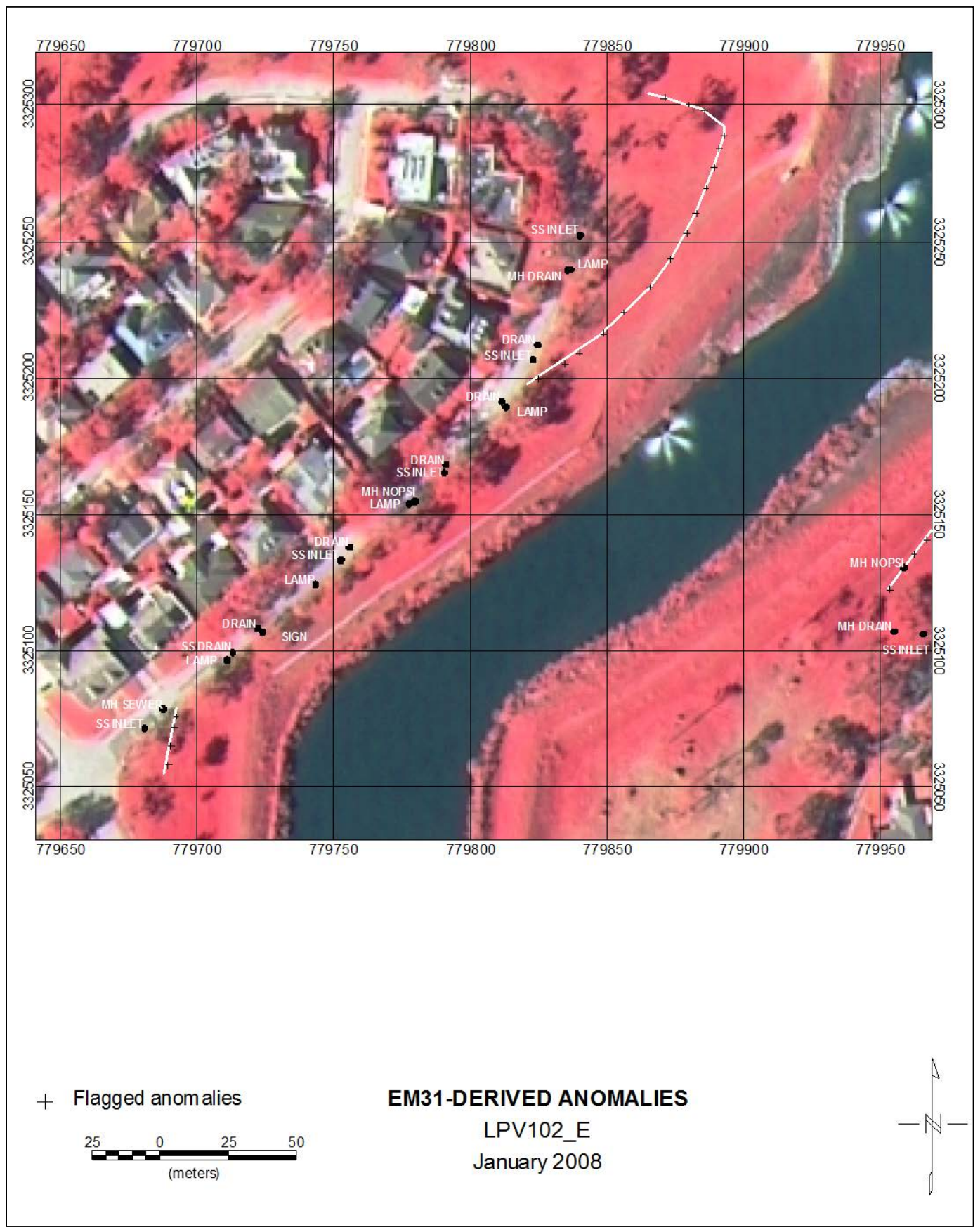

\section{LPV103}

LPV103 is located between the east bank of the Orleans Avenue Canal and the west bank of the London Avenue Canal along Lakeshore Drive. The site is divided into 12 sections designated LPV103_AA through LPV103_K for presentation purposes (Figure 16). The anomalies for LPV103_AA through LPV103_K based on the results of the EM31 surveys are presented in 
Figures 17 through 28, respectively. The locations of the flagged anomalies for LPV103 are presented in Appendix A, Table A3. The EM31 conductivity and in-phase maps for LPV103 are shown in Appendix B, Figures B17 through B40.

\section{LPV104}

LPV104 is located between the west bank of the London Avenue Canal and the western approach of the Senator Ted Hickey Bridge. The site is divided into 11 sections designated LPV104_A through LPV104_K for presentation purposes (Figure 29). The anomalies for LPV104_A through LPV104_K based on the results of the EM31 surveys are shown in Figures 30 through 40, respectively. The locations of the flagged anomalies for LPV104 are presented in Appendix A, Table A4. The EM31 conductivity and in-phase maps for LPV104 are shown in Appendix B, Figures B41 through B62.

Early in the study, a trench was excavated across a linear EM31 anomaly at LPV104_K to verify the effectiveness of the geophysical surveys in locating buried utilities (Figure 41). A multi-strand copper cable approximately $1.25 \mathrm{~cm}$ ( $0.5 \mathrm{in}$.) in diameter was located at a depth of approximately $0.6 \mathrm{~m}$ (2 ft), as shown in Figure 42.

HPO personnel were also interested in determining the path of a storm sewer located between Leon C. Simon Drive and an access road adjacent to and east of Leroy J ohnson Drive (LPV104_K). HPO personnel assumed that the sewer drained into a sewer line running along Leon C. Simon Drive, but did not know where or if they connected. The Universal Transverse Mercator System (UTM) coordinates of the storm sewer grate are (785524.6, 3325885.6) and is noted in Figure 40 as "SEWER1." Initially, HPO personnel tried to map the path of the storm sewer by making a series of excavations down to the storm sewer pipe at selected locations along an assumed path. The first two excavations were located approximately $10 \mathrm{~m}$ (33 ft) and $20 \mathrm{~m}$ (66 ft) southeast of the sewer grate and successfully located the pipe (Figure 40). It appeared that the projected sewer line path was crossing underneath the levee. A third excavation, approximately $60 \mathrm{~m}$ (200 ft) from the sewer grate, was dug to a depth of approximately $2.5 \mathrm{~m}$ (8 ft), but it failed to locate the pipe. 


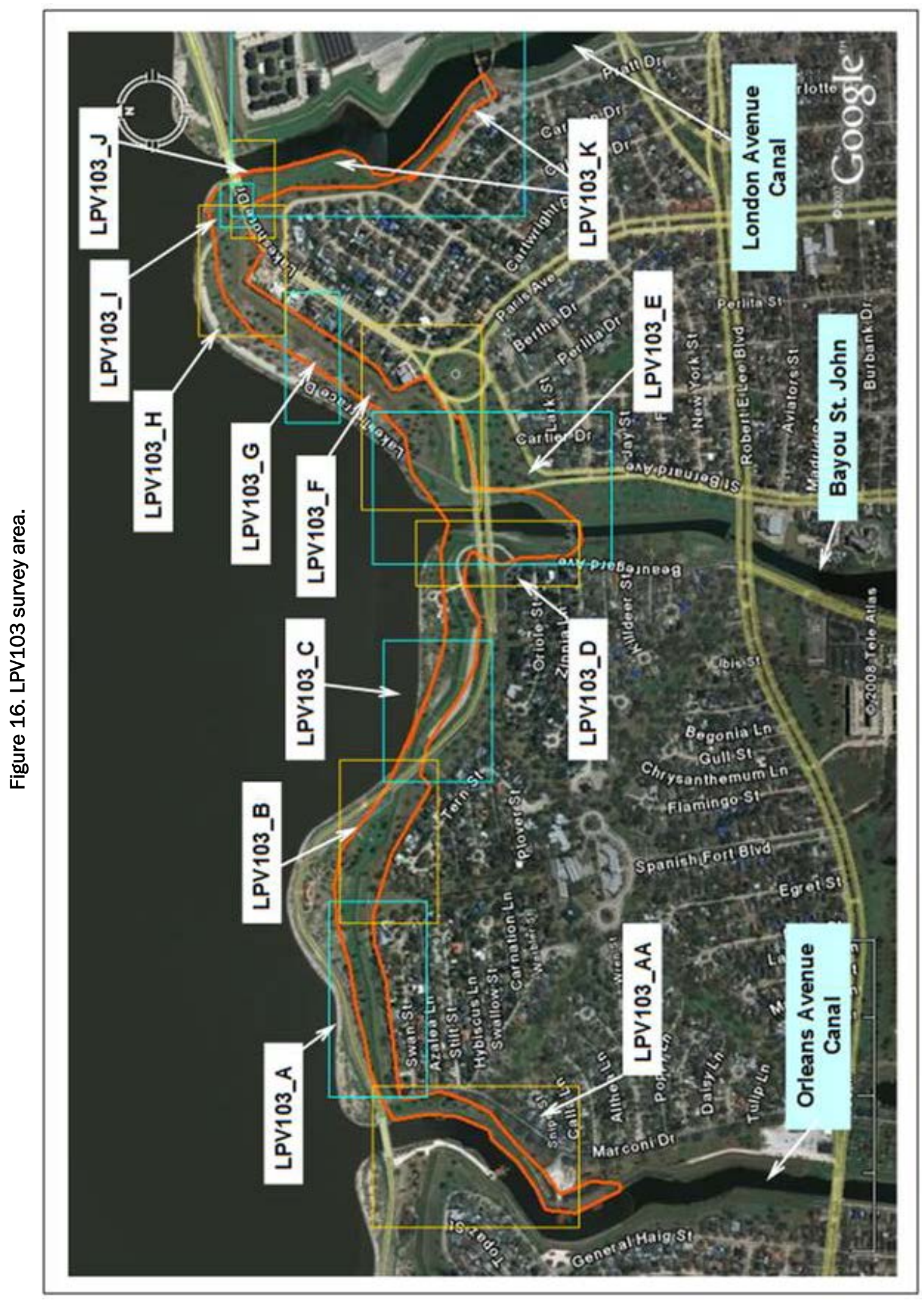


Figure 17. Anomalies interpreted from EM31 survey, LPV103_A.A.

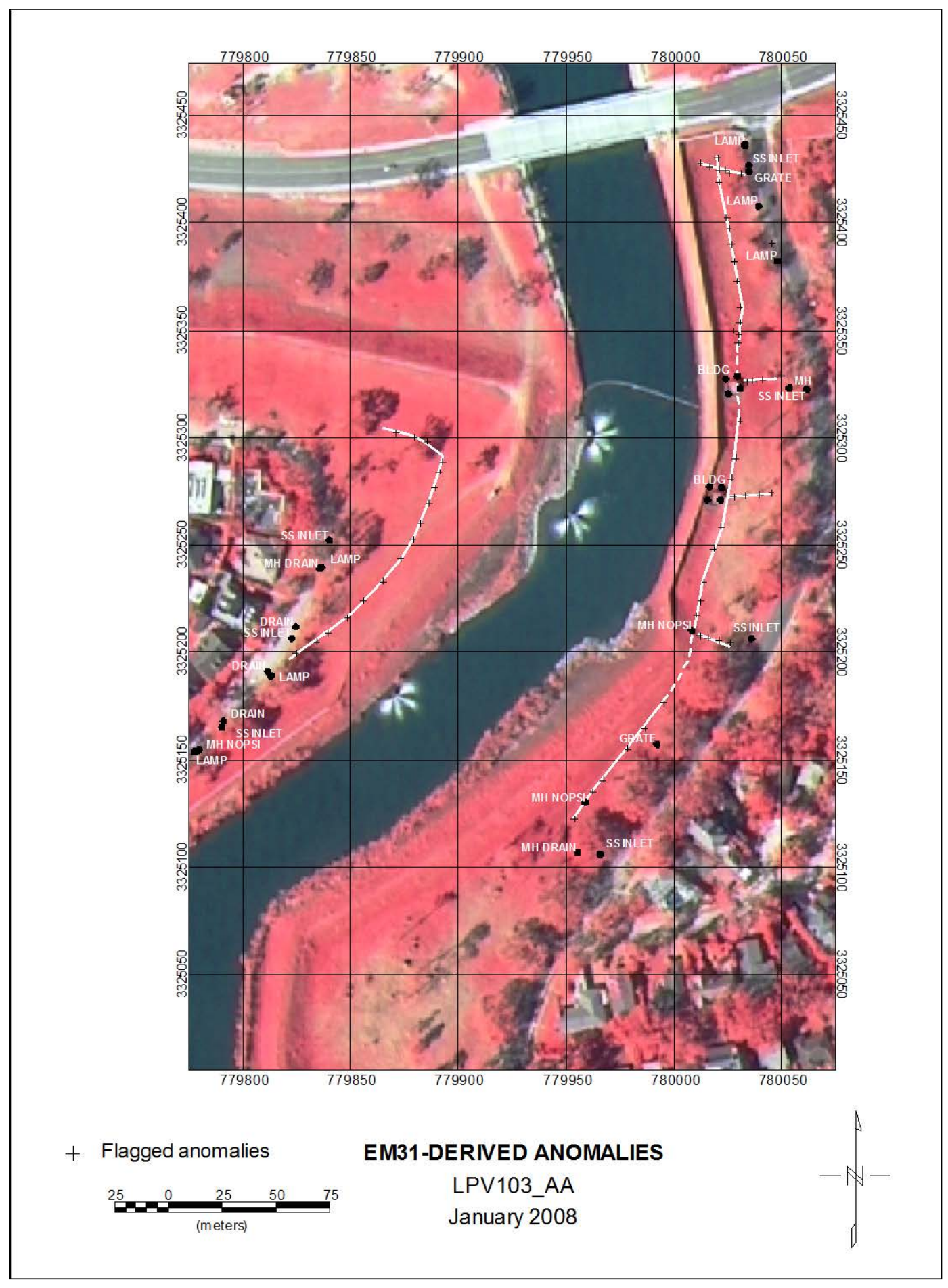




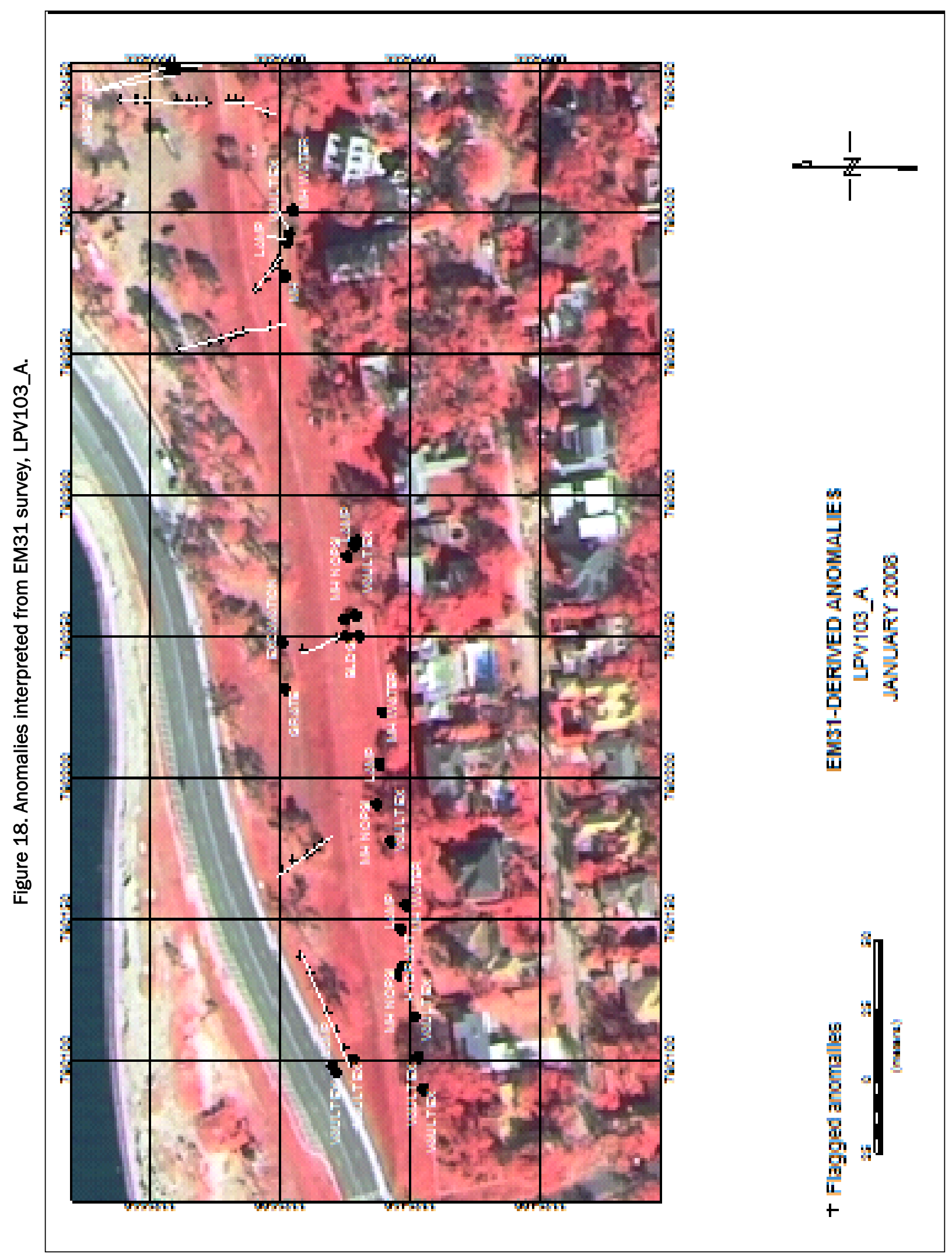




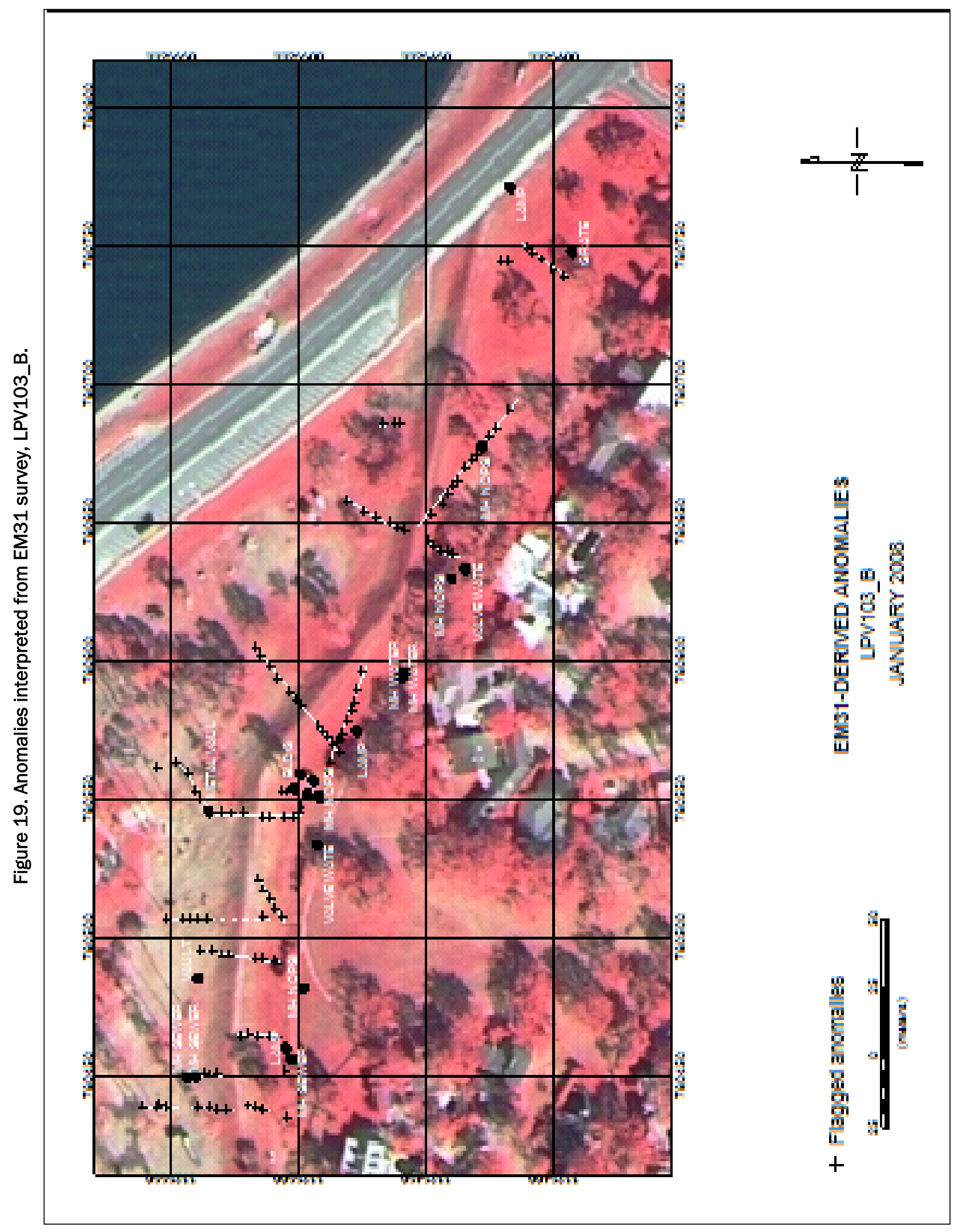




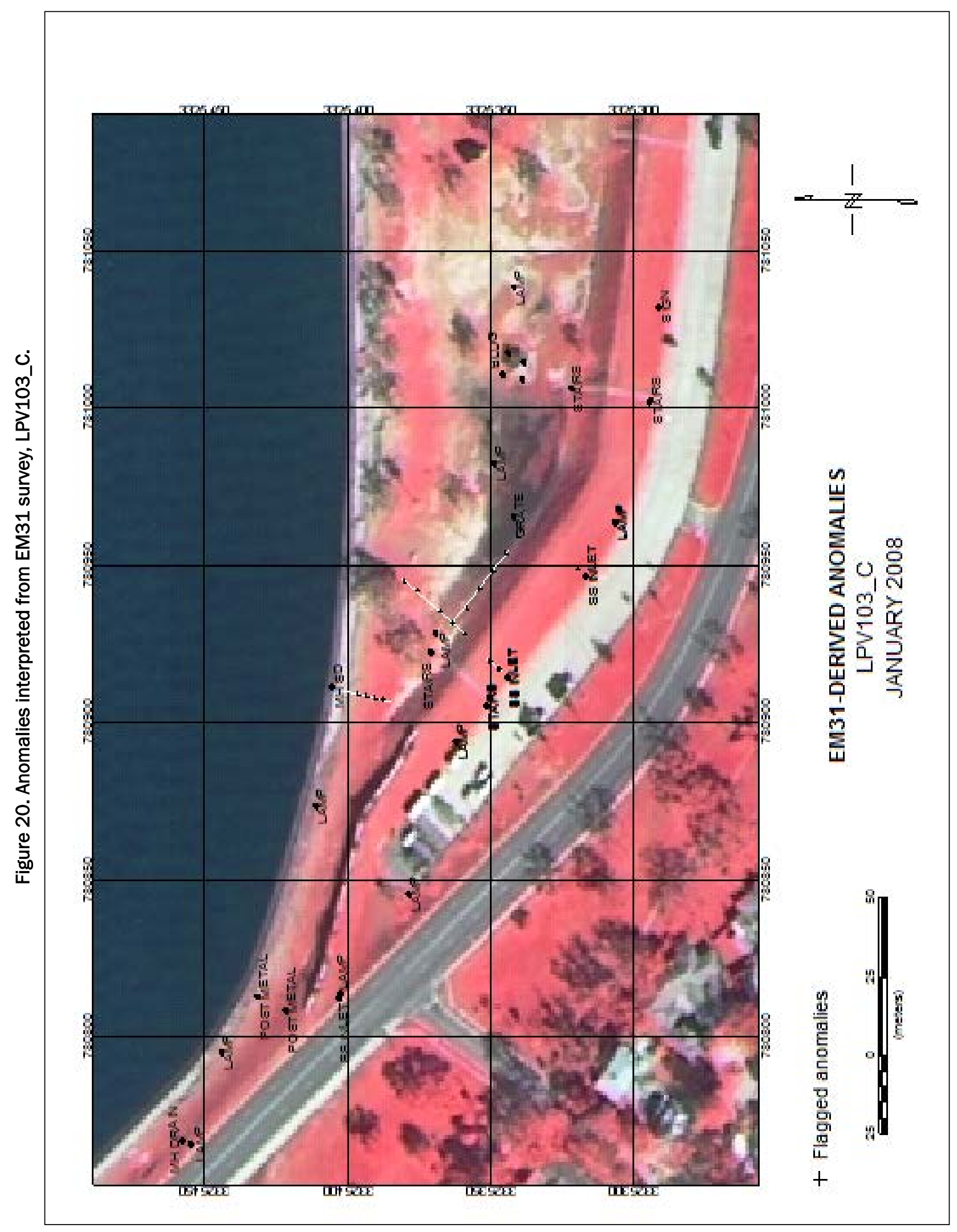


Figure 21. Anomalies interpreted from EM31 survey, LPV103_D.

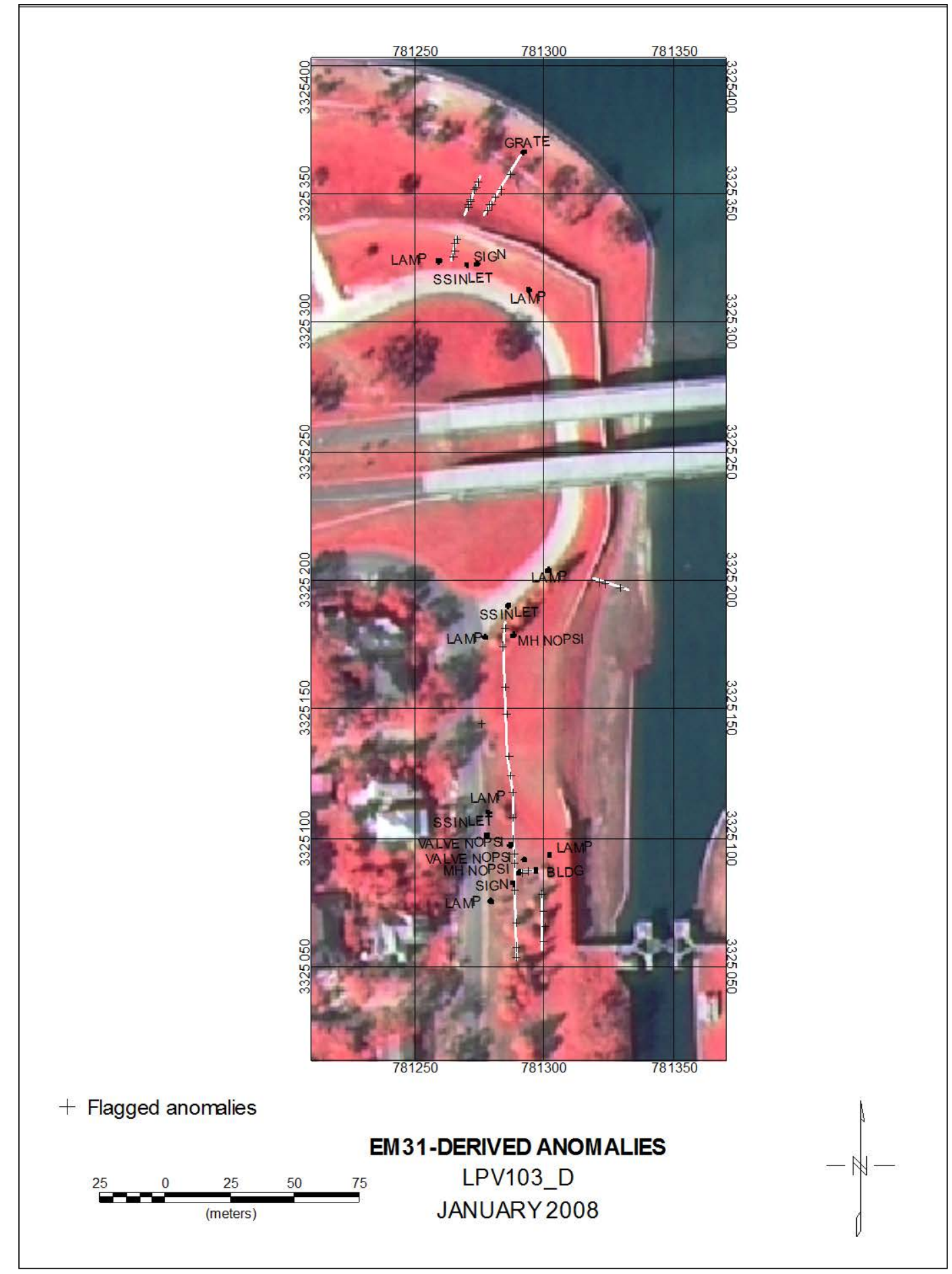


Figure 22. Anomalies interpreted from EM31 survey, LPV103_E.

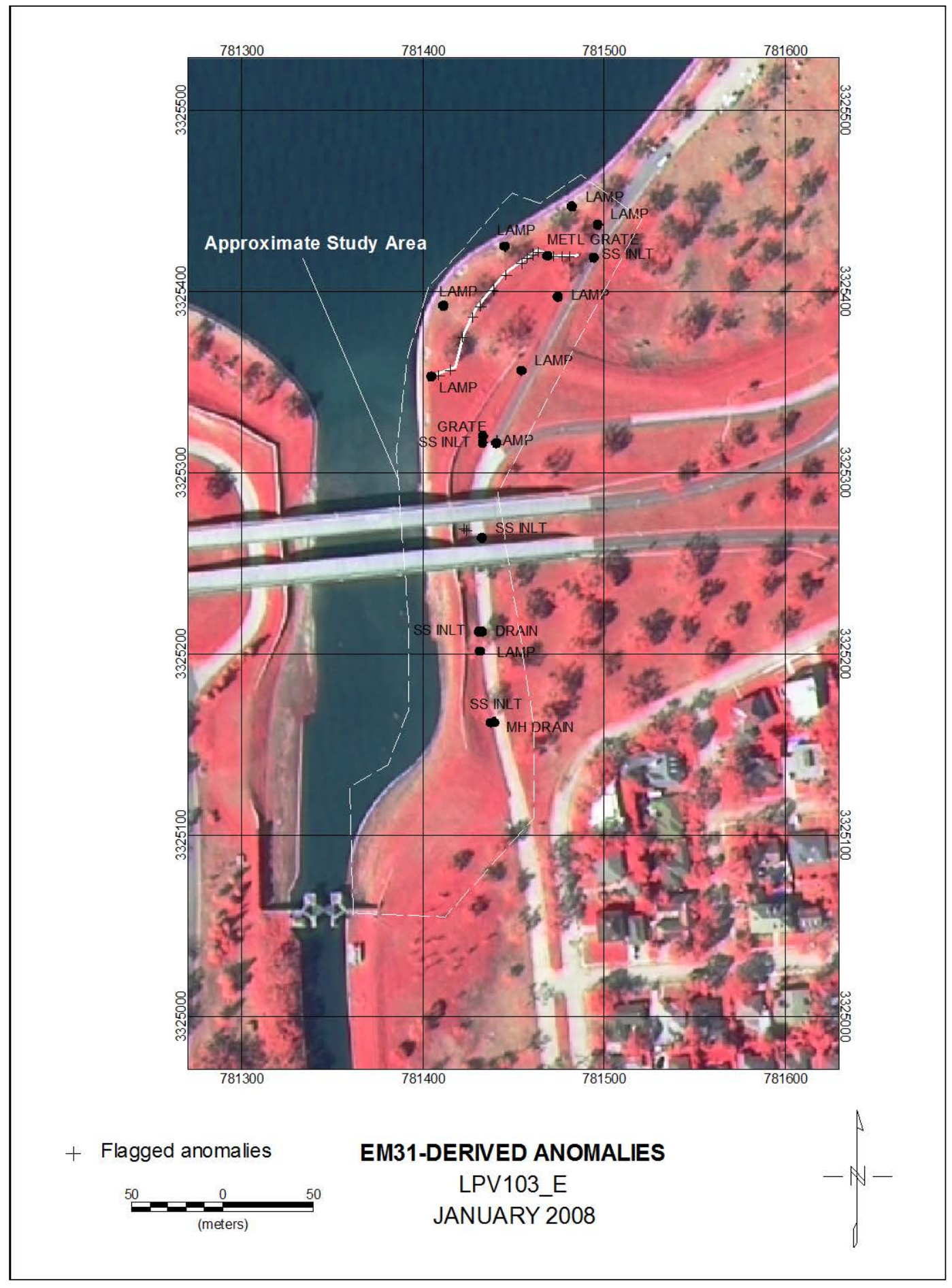




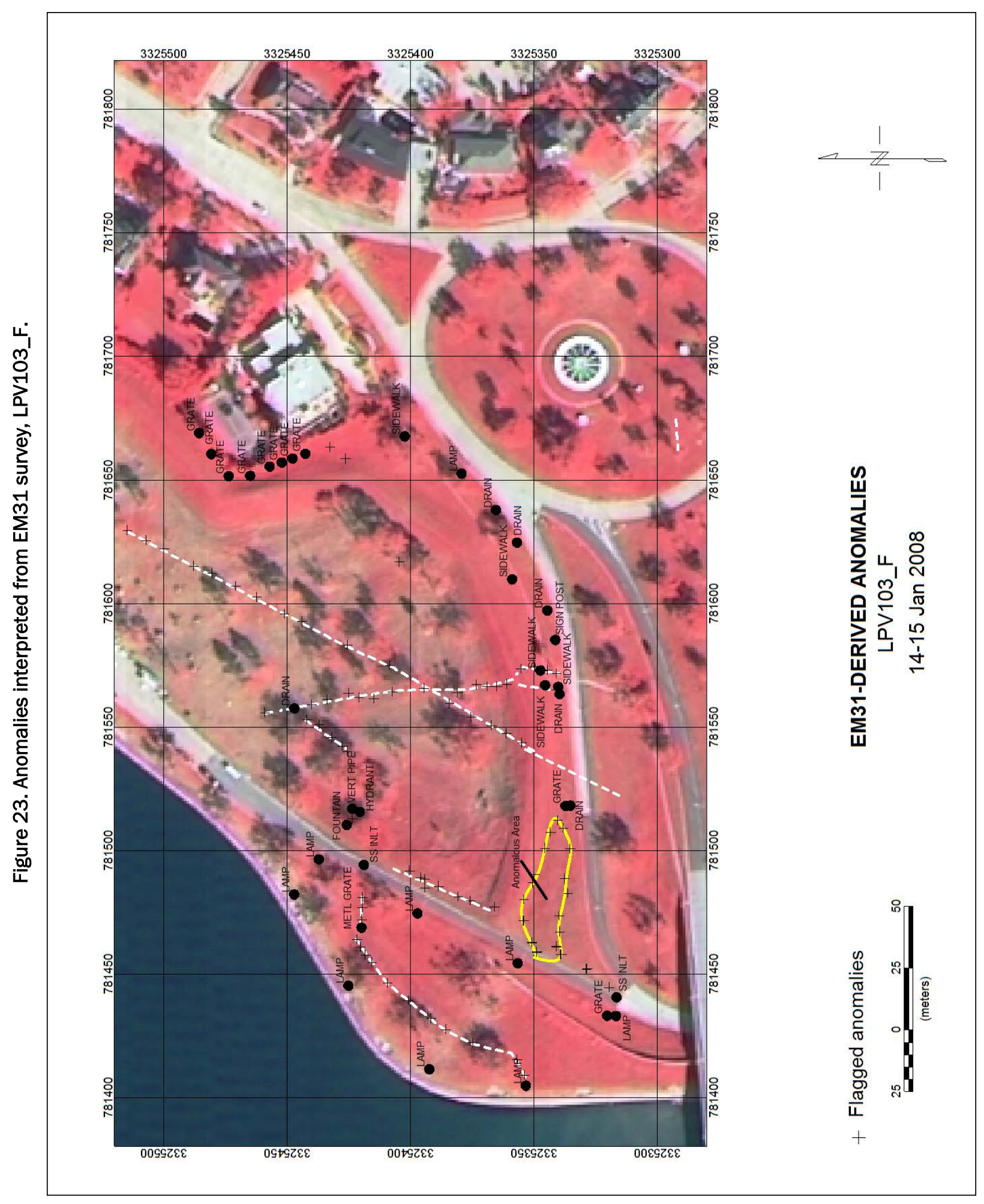




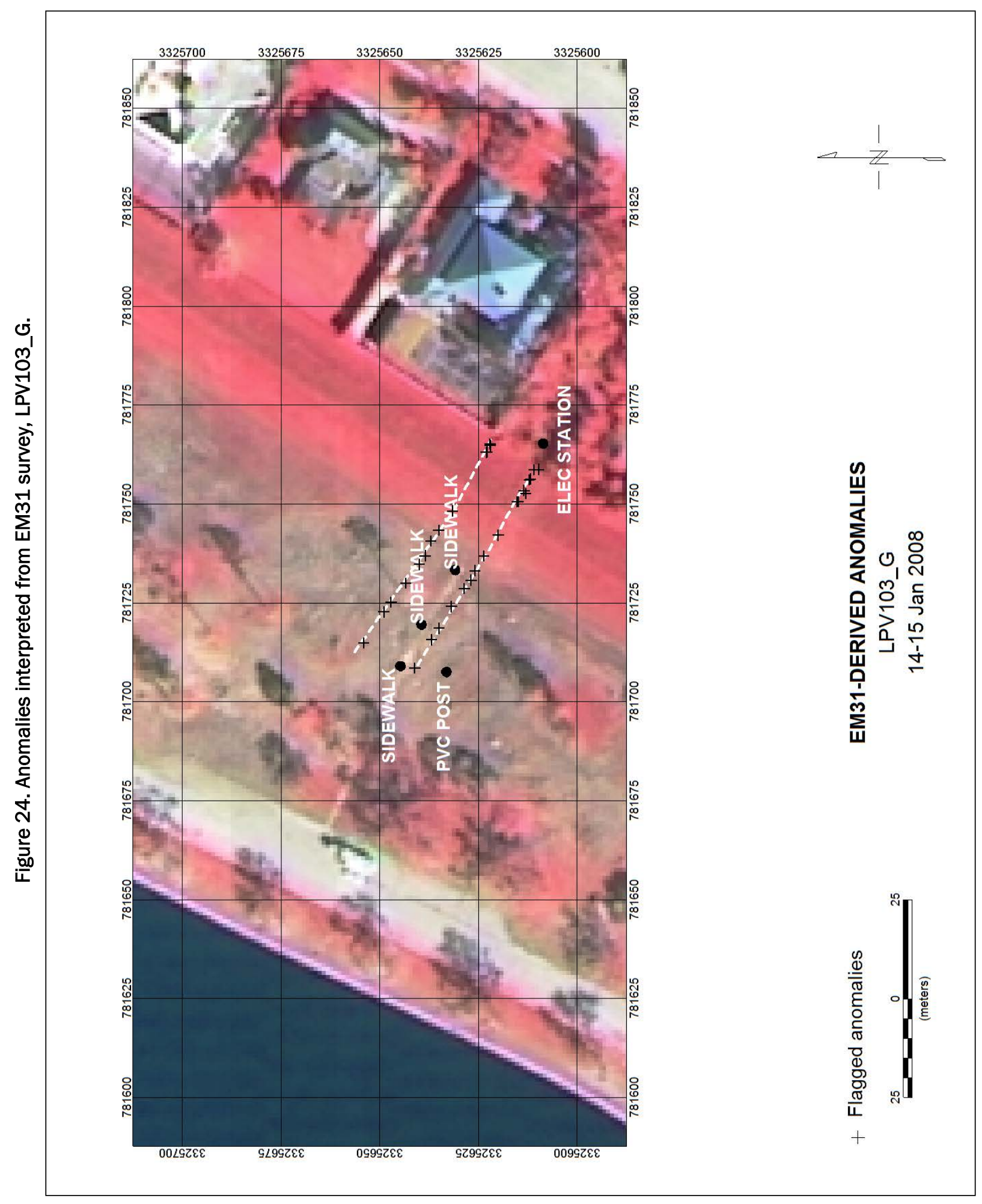




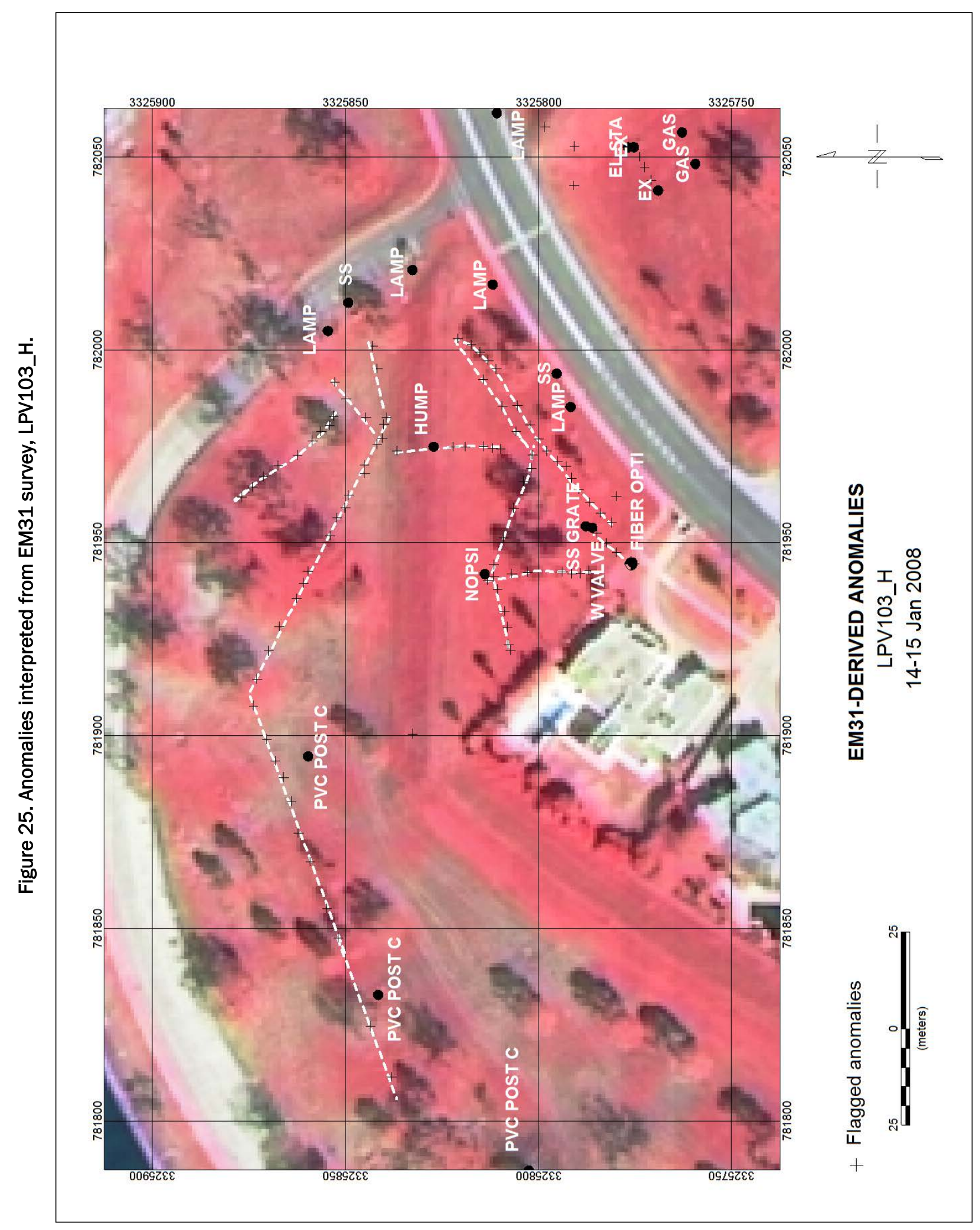




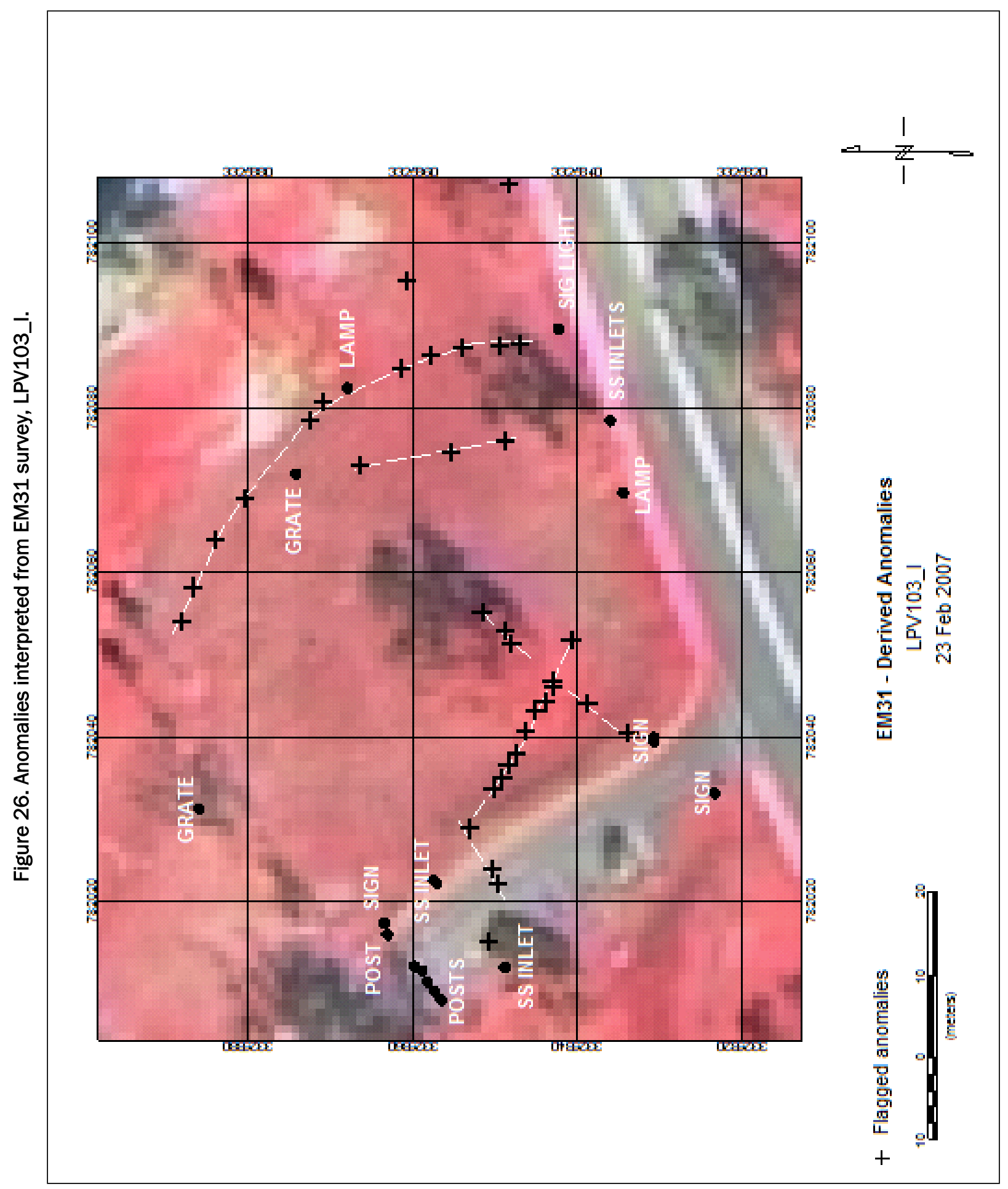




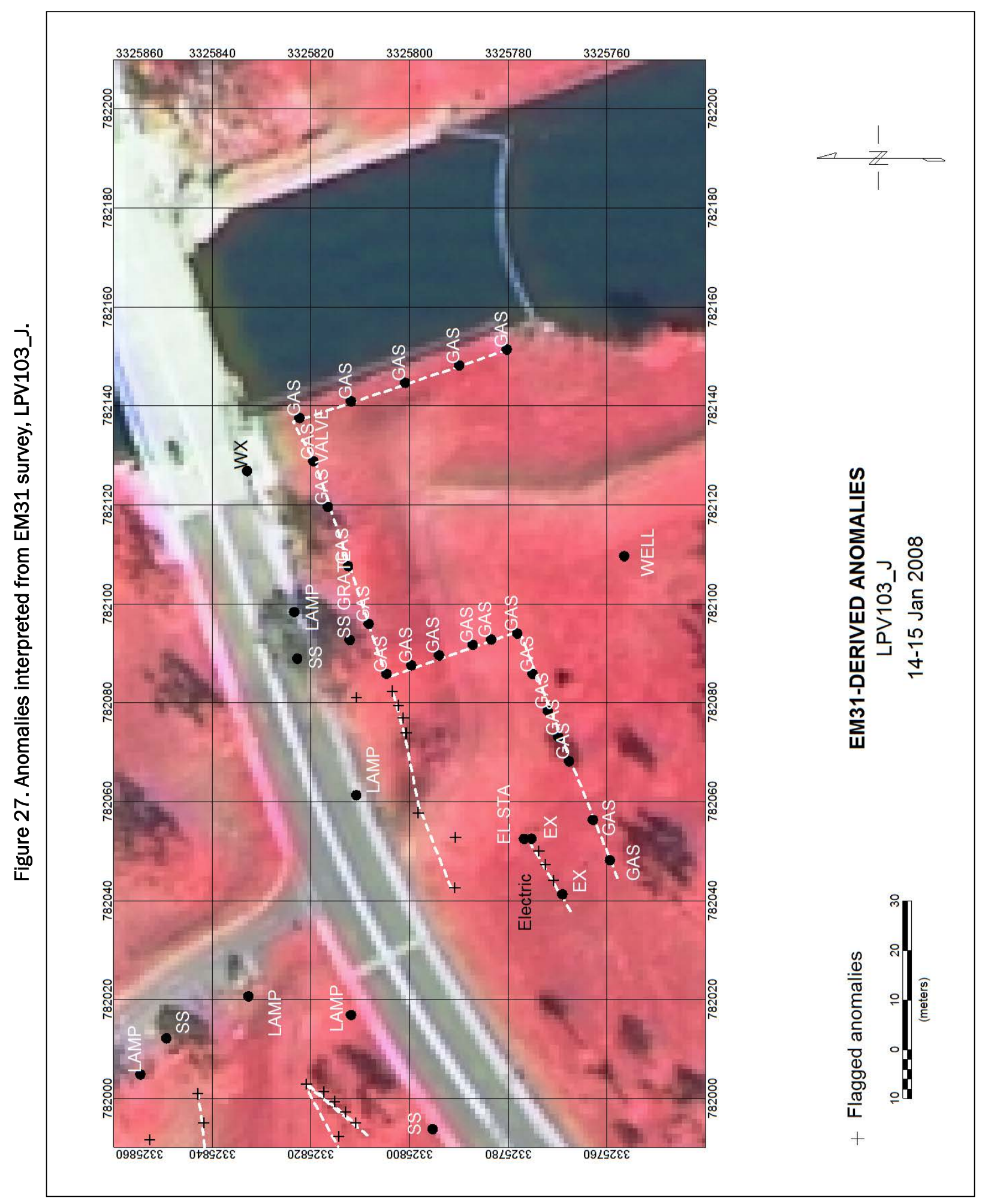


Figure 28. Anomalies interpreted from EM31 survey, LPV103_K.

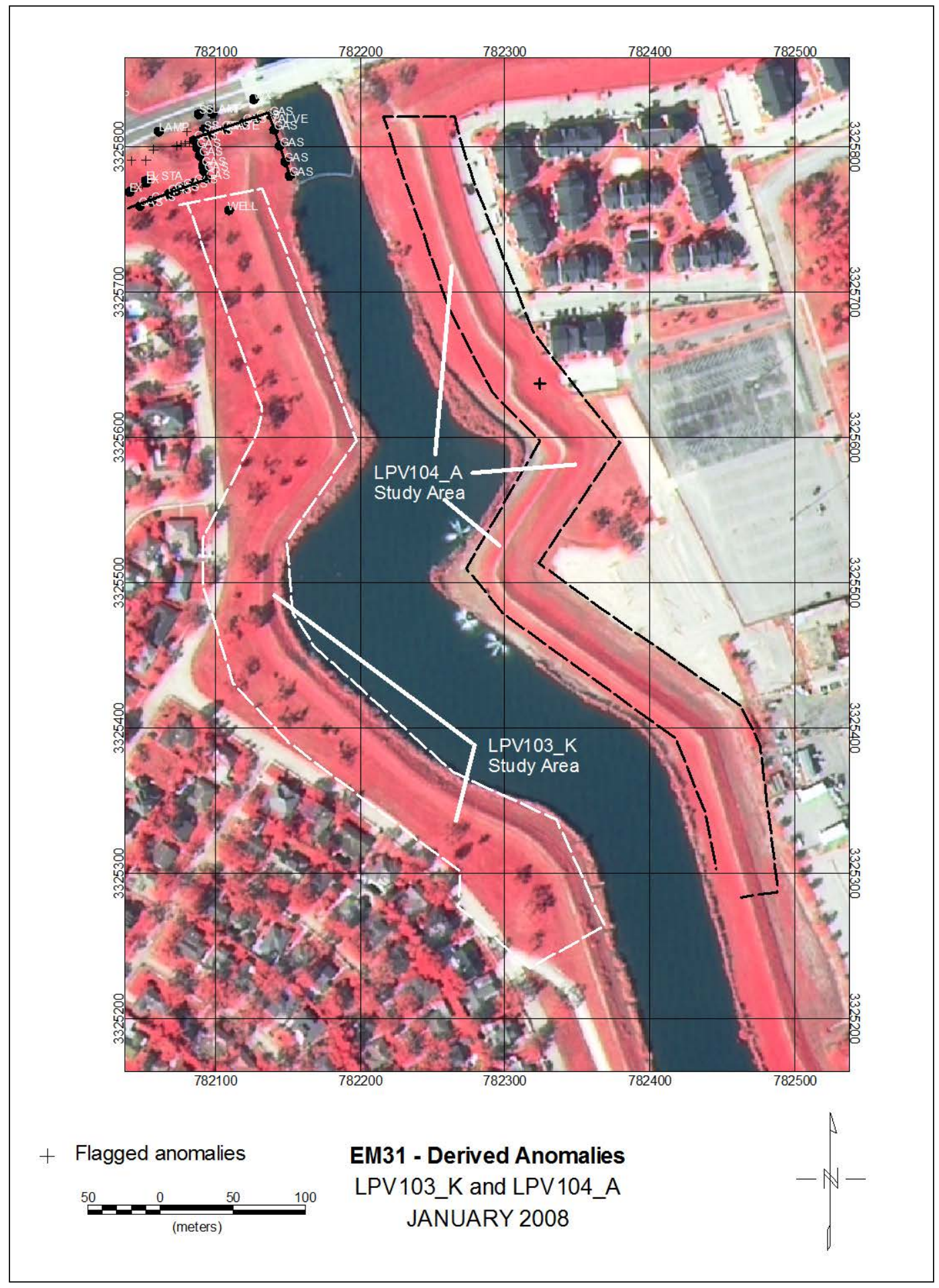




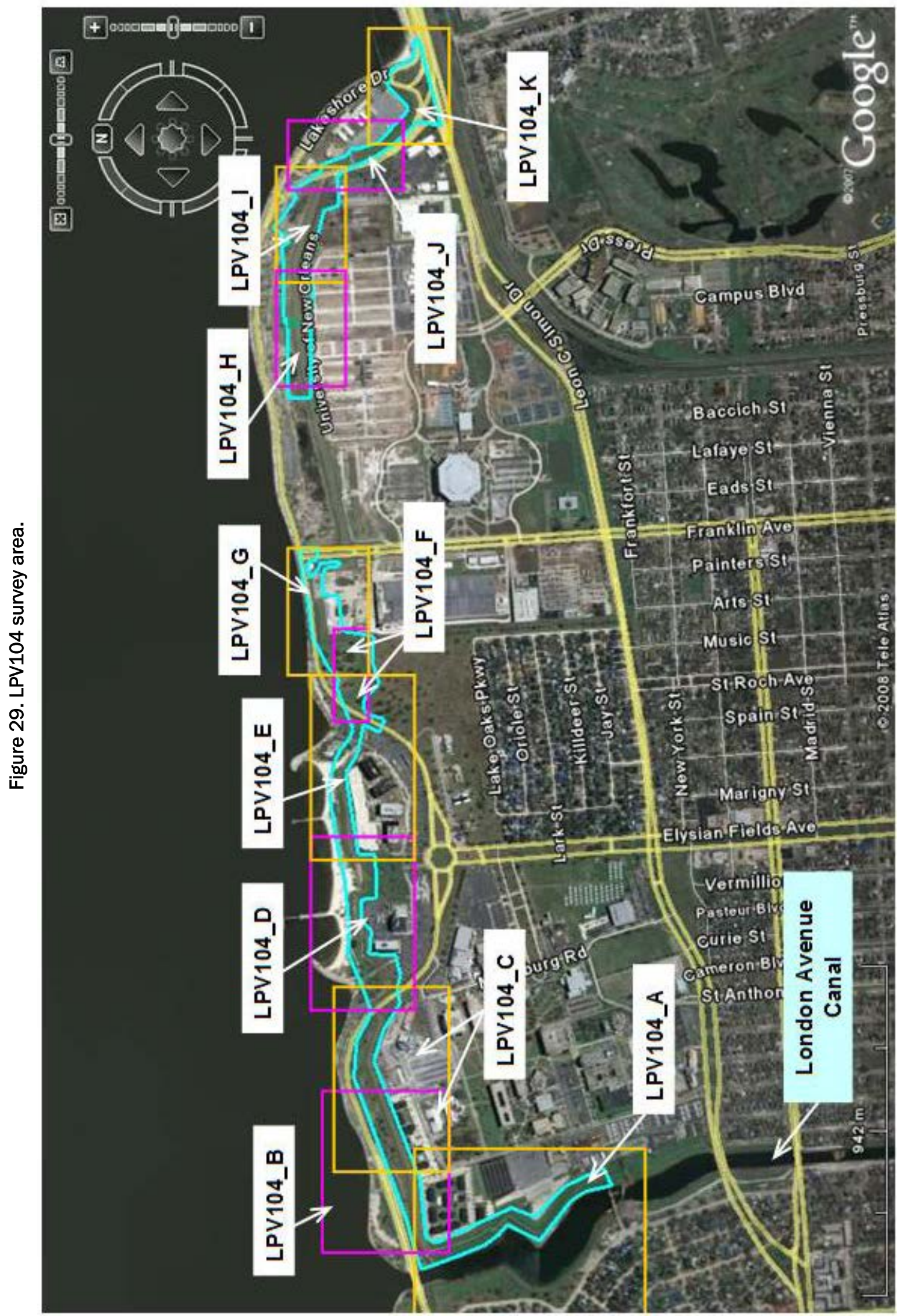


Figure 30. Anomalies interpreted from EM31 survey, LPV104_A.

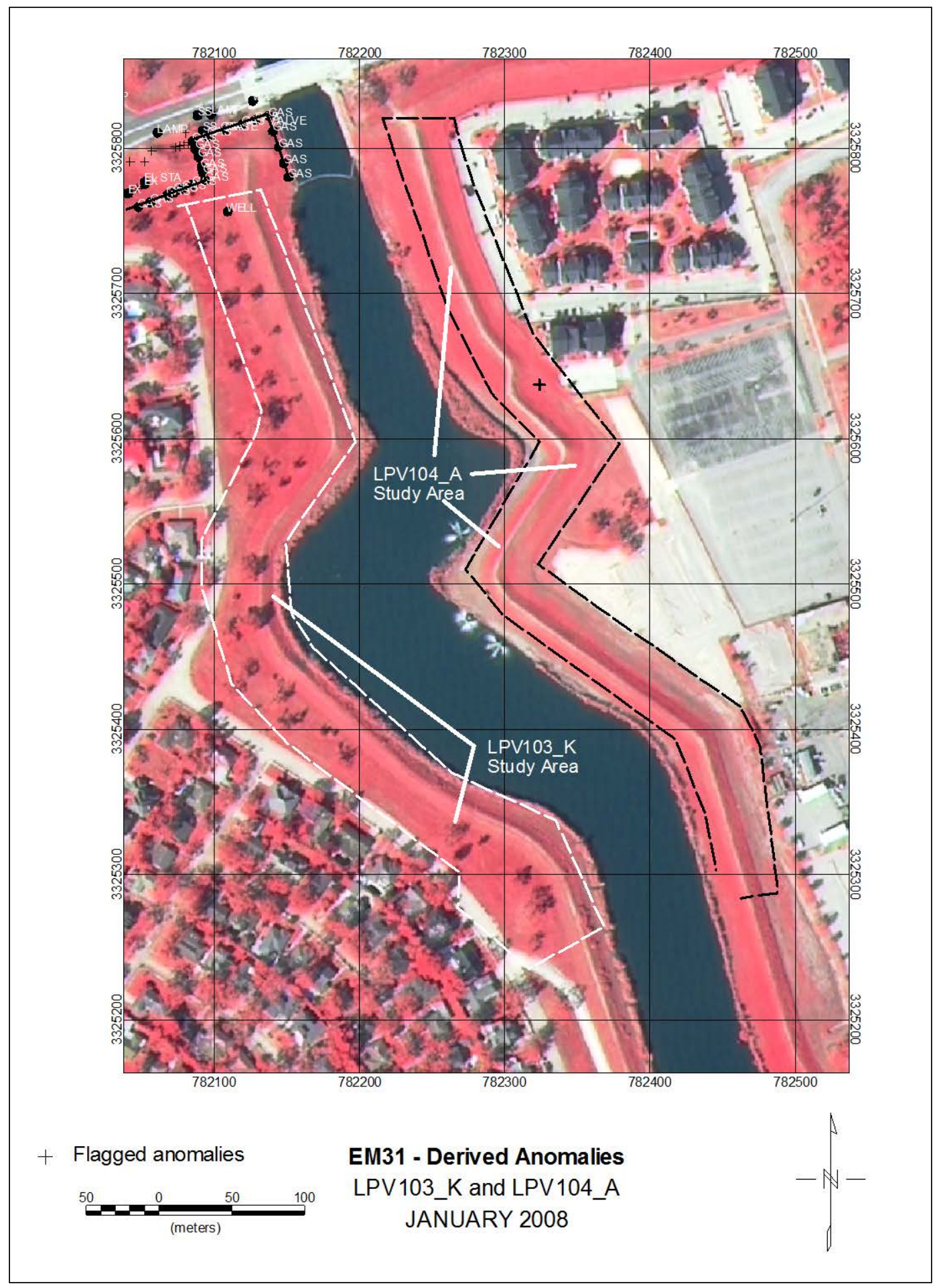




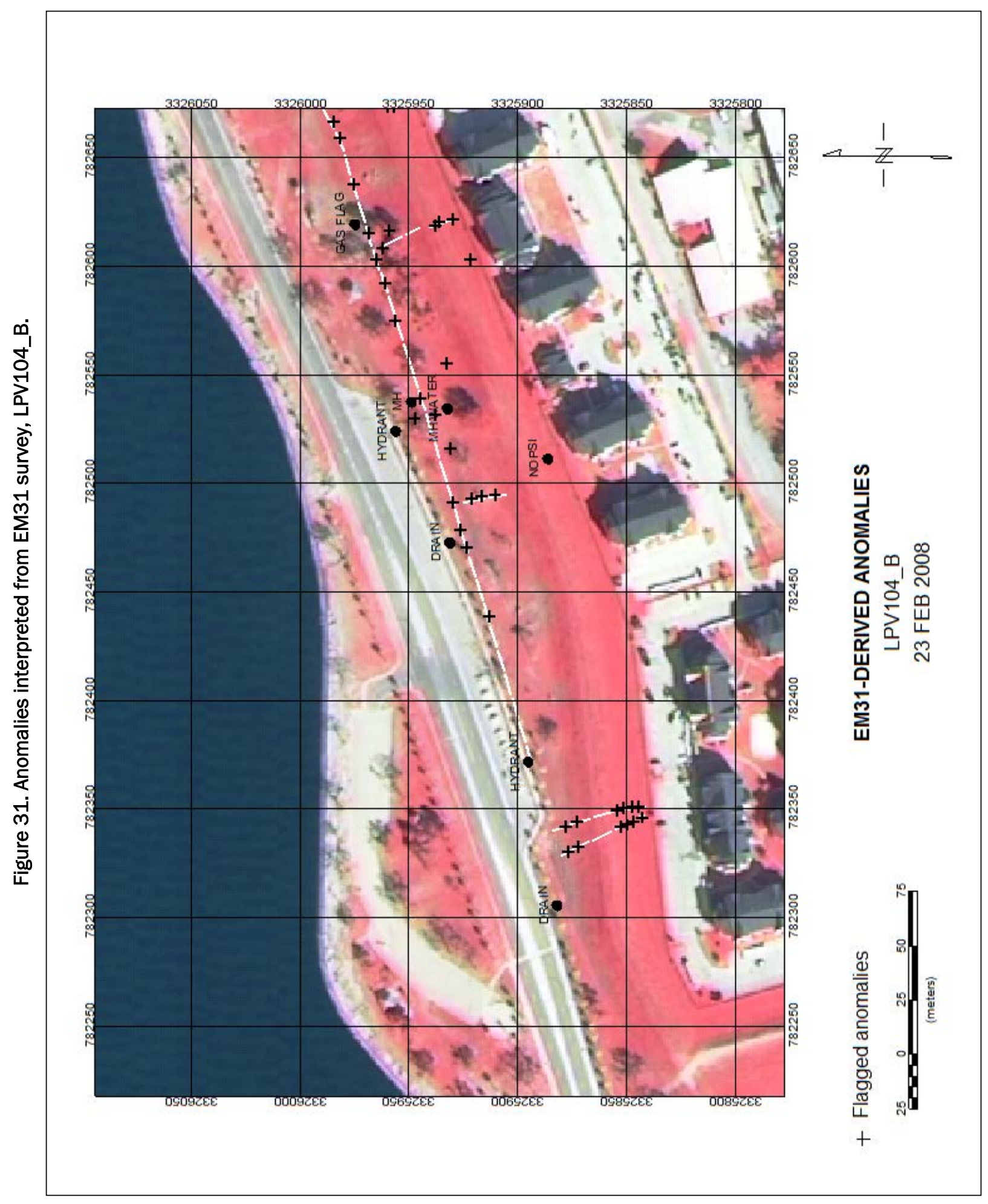




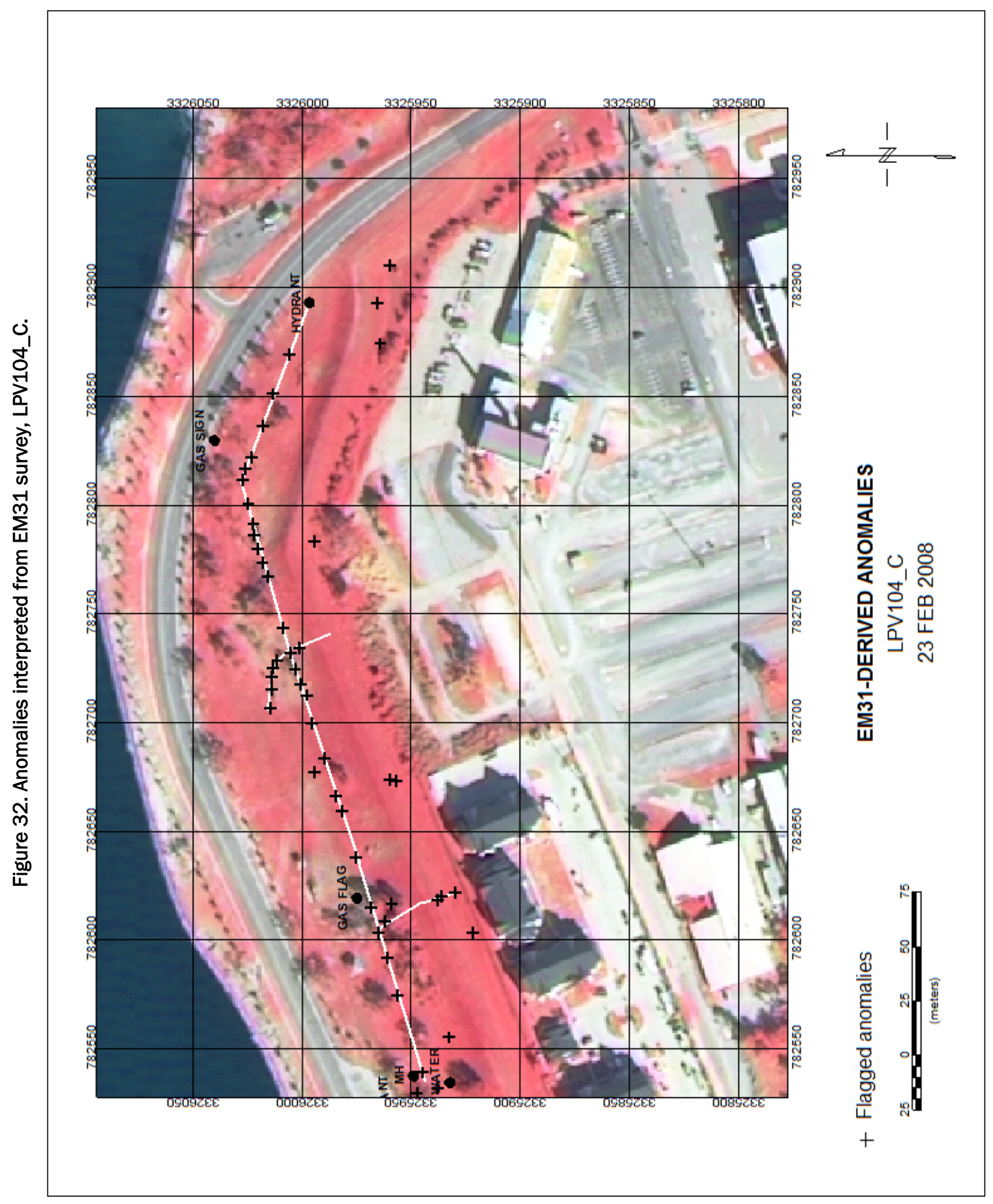




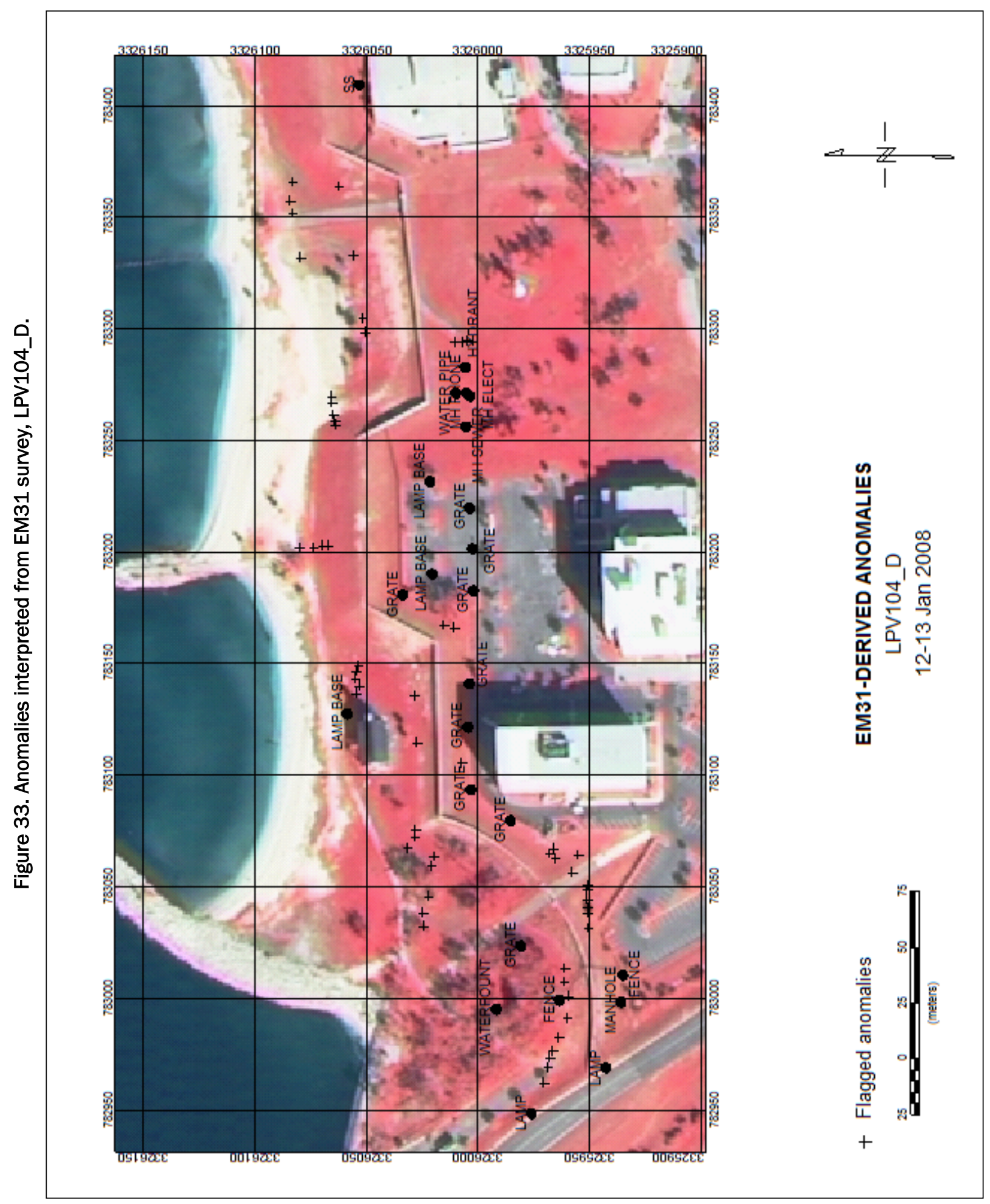




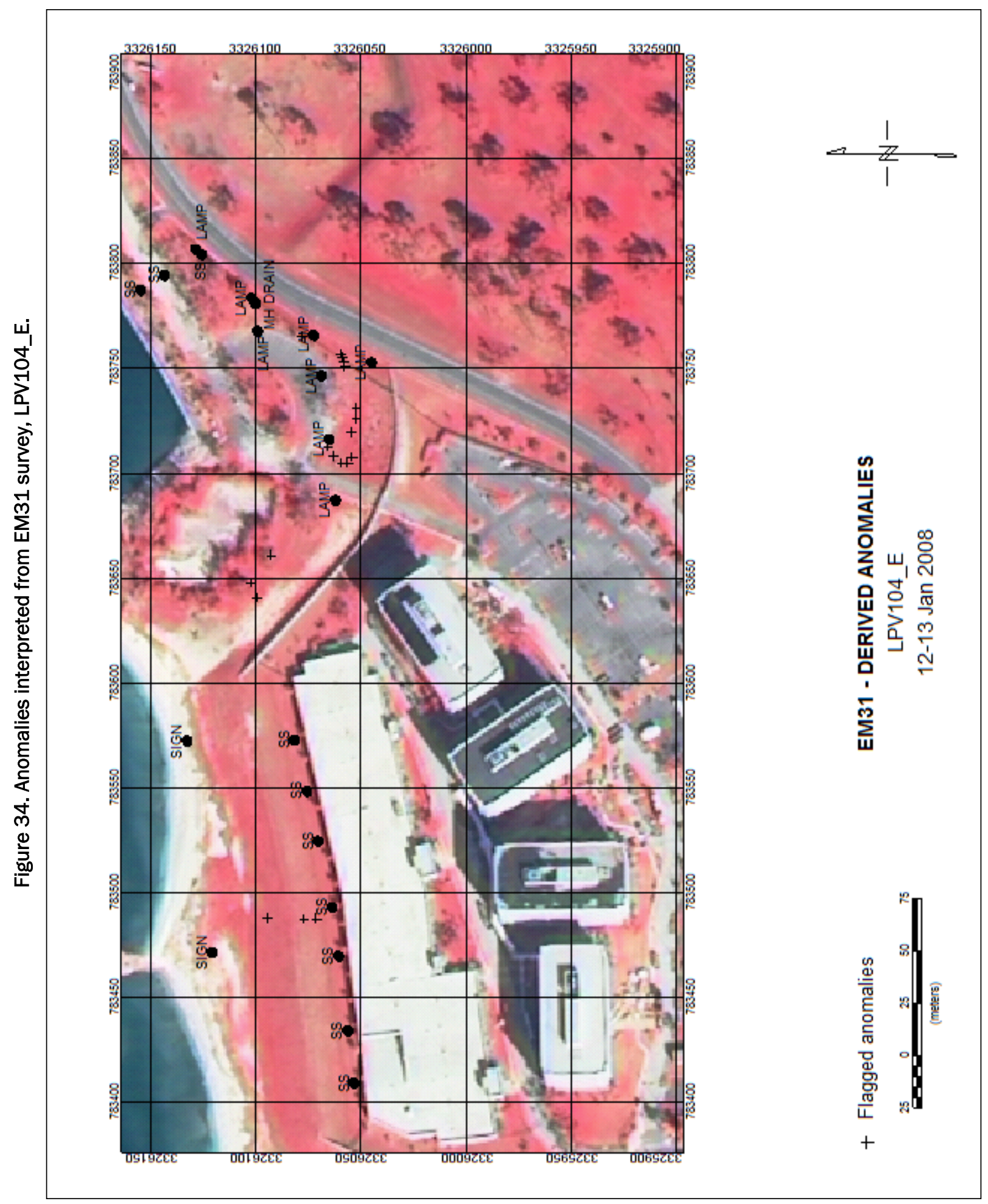




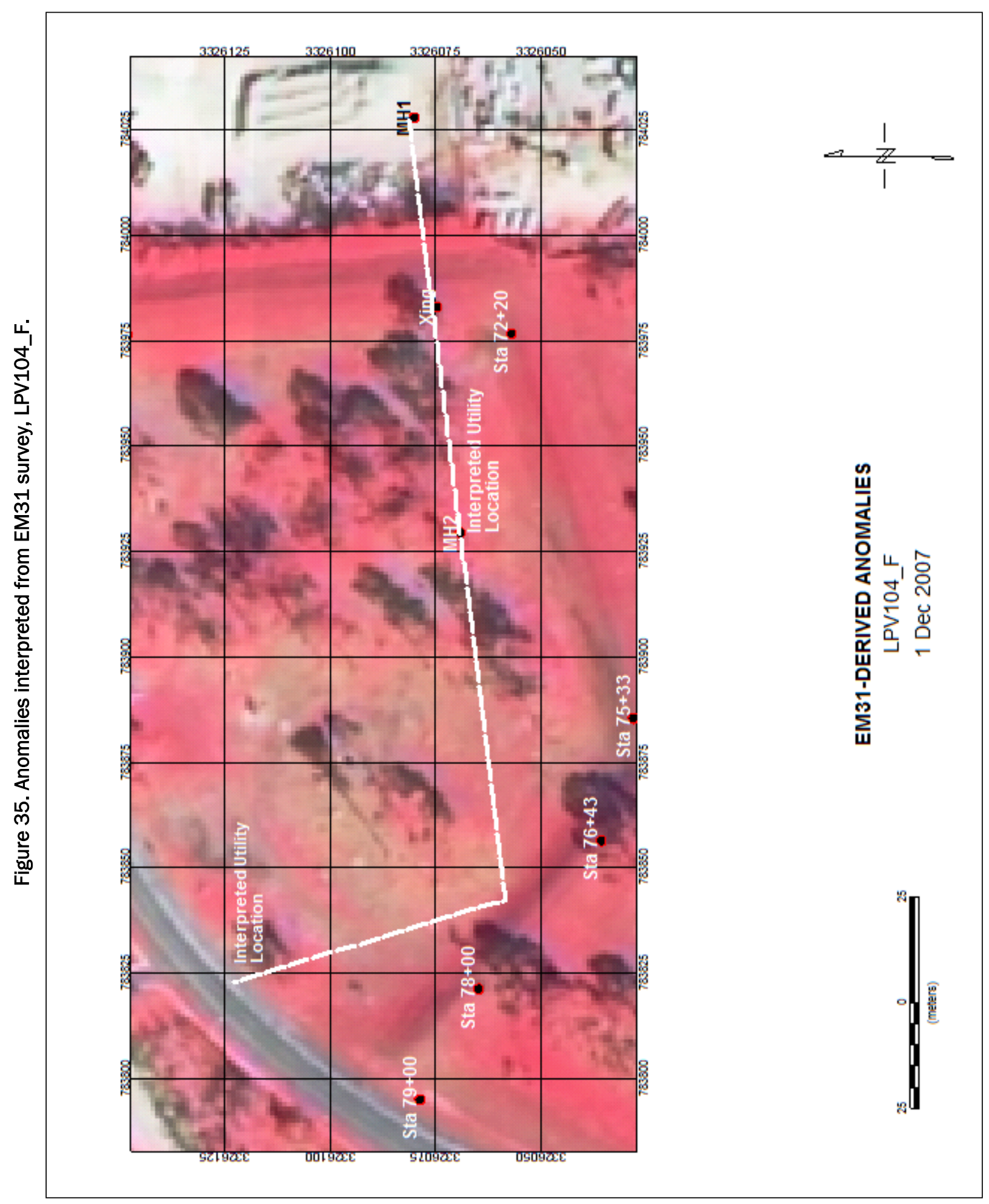




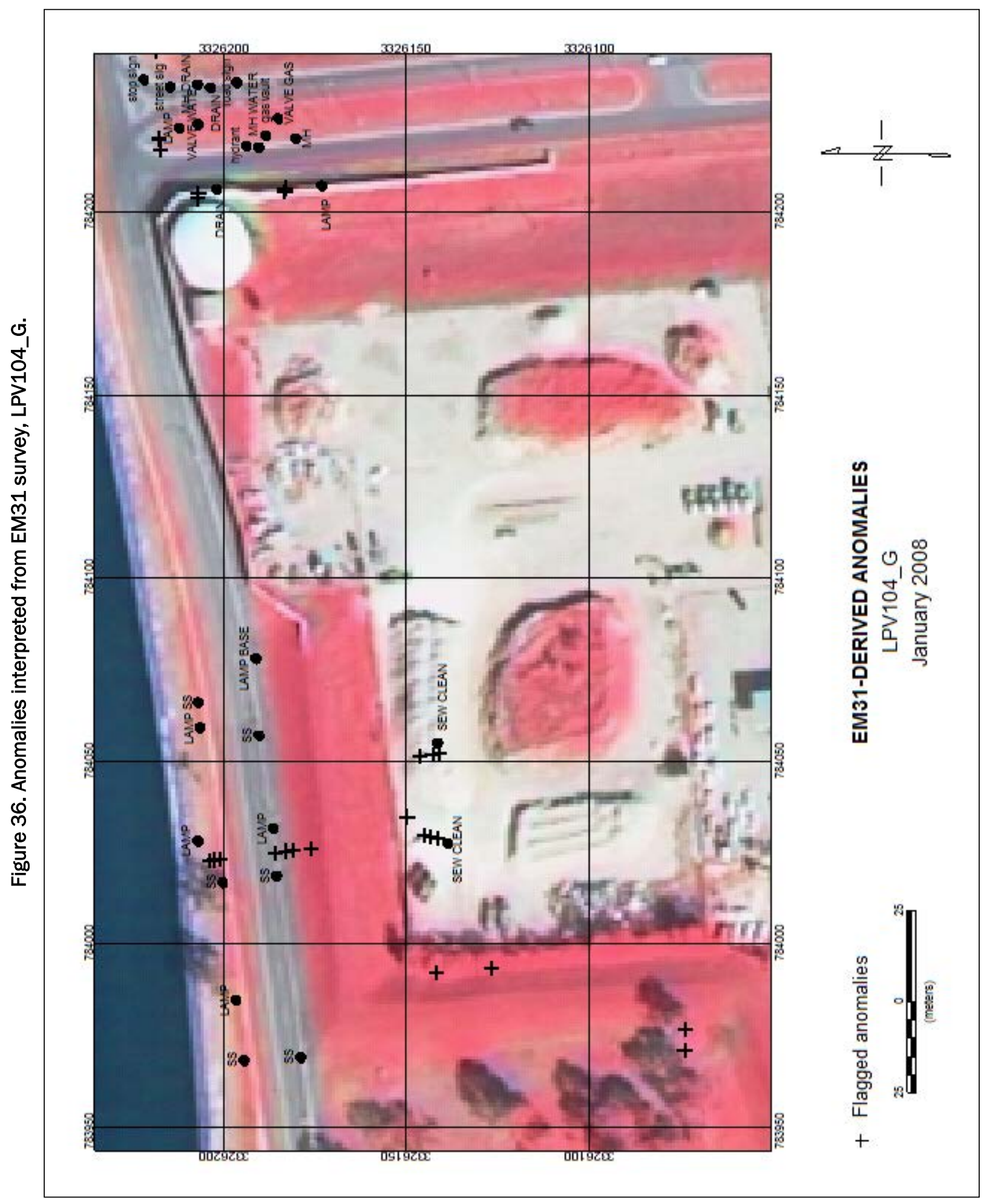




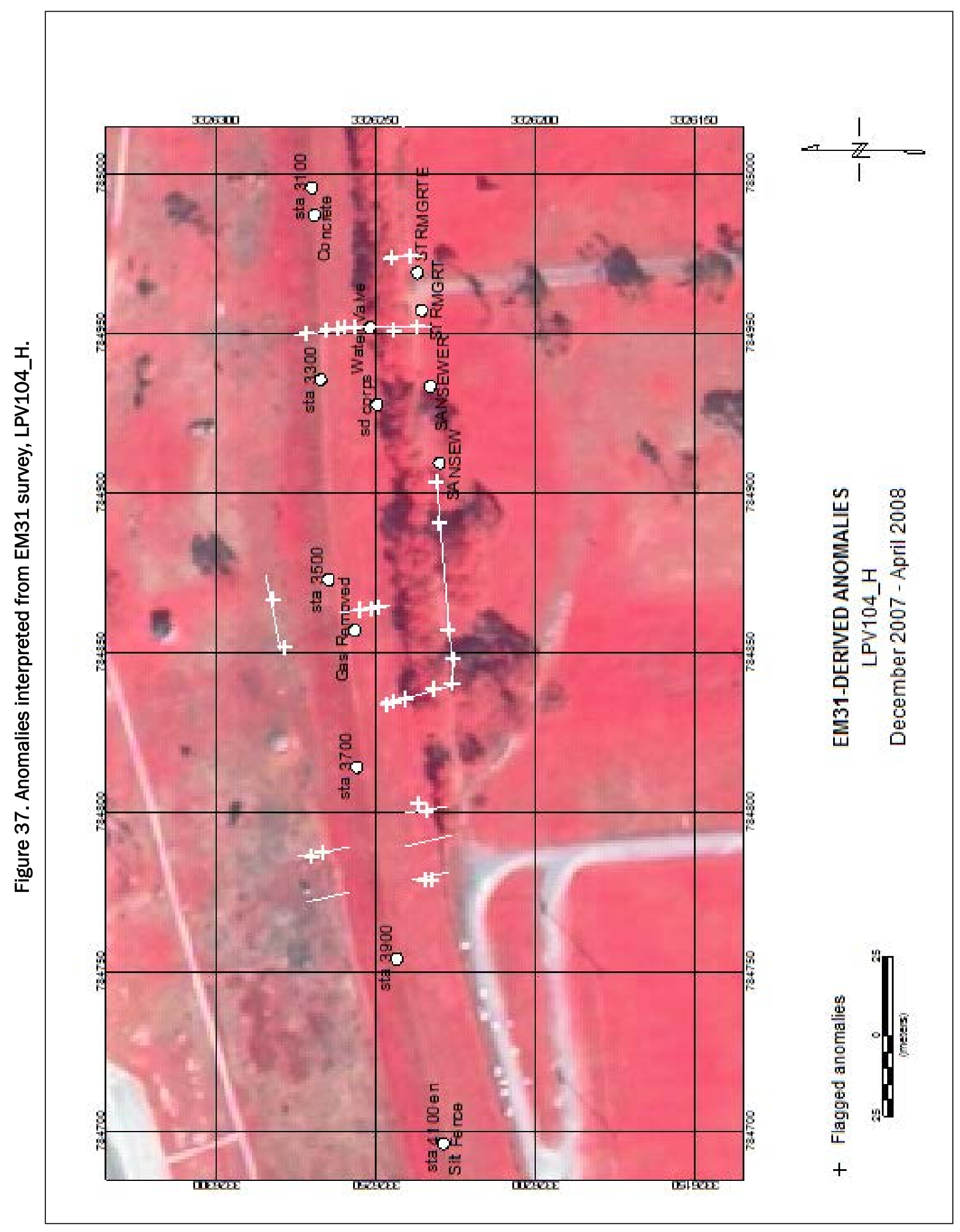




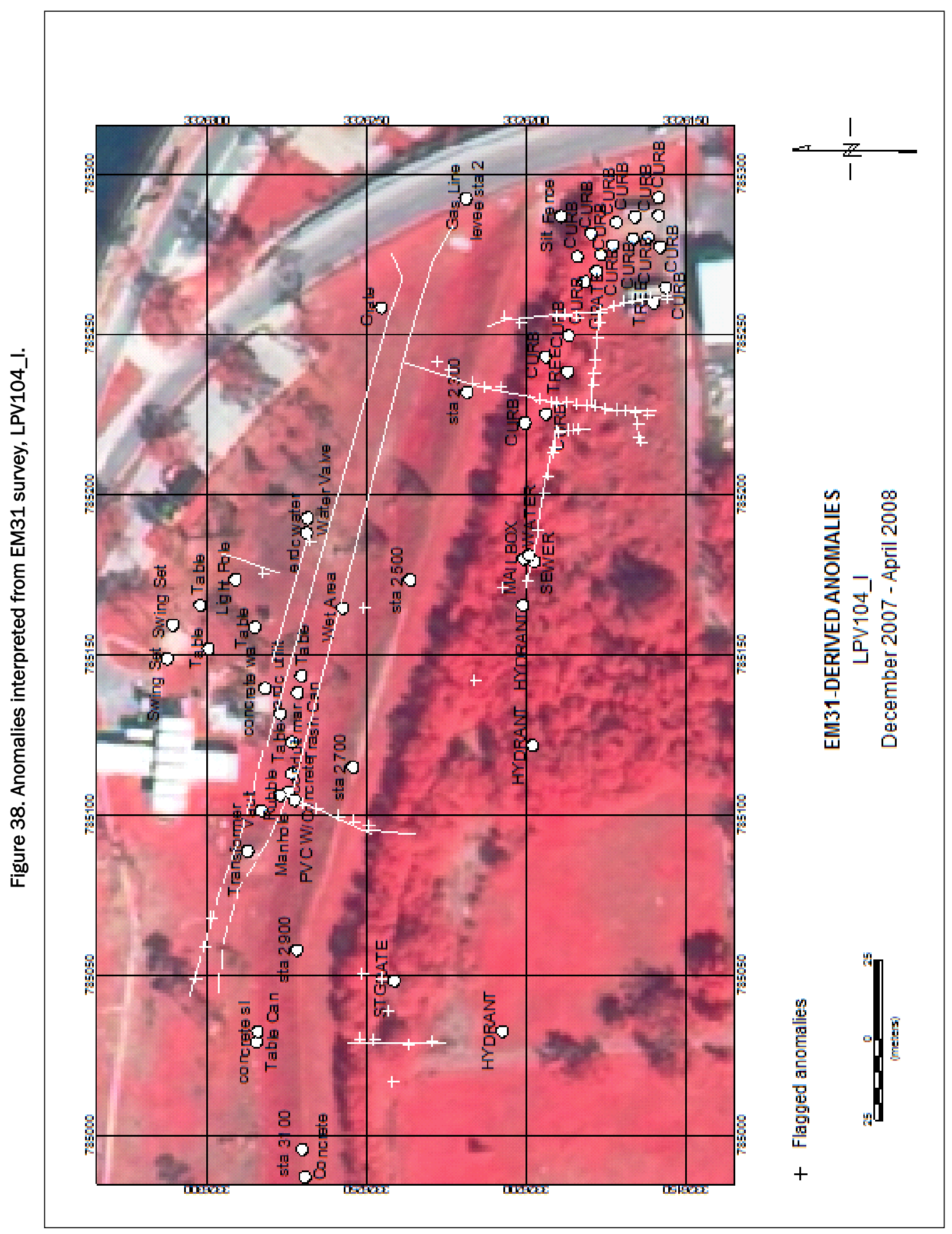


Figure 39. Anomalies interpreted from EM31 survey, LPV104_J.

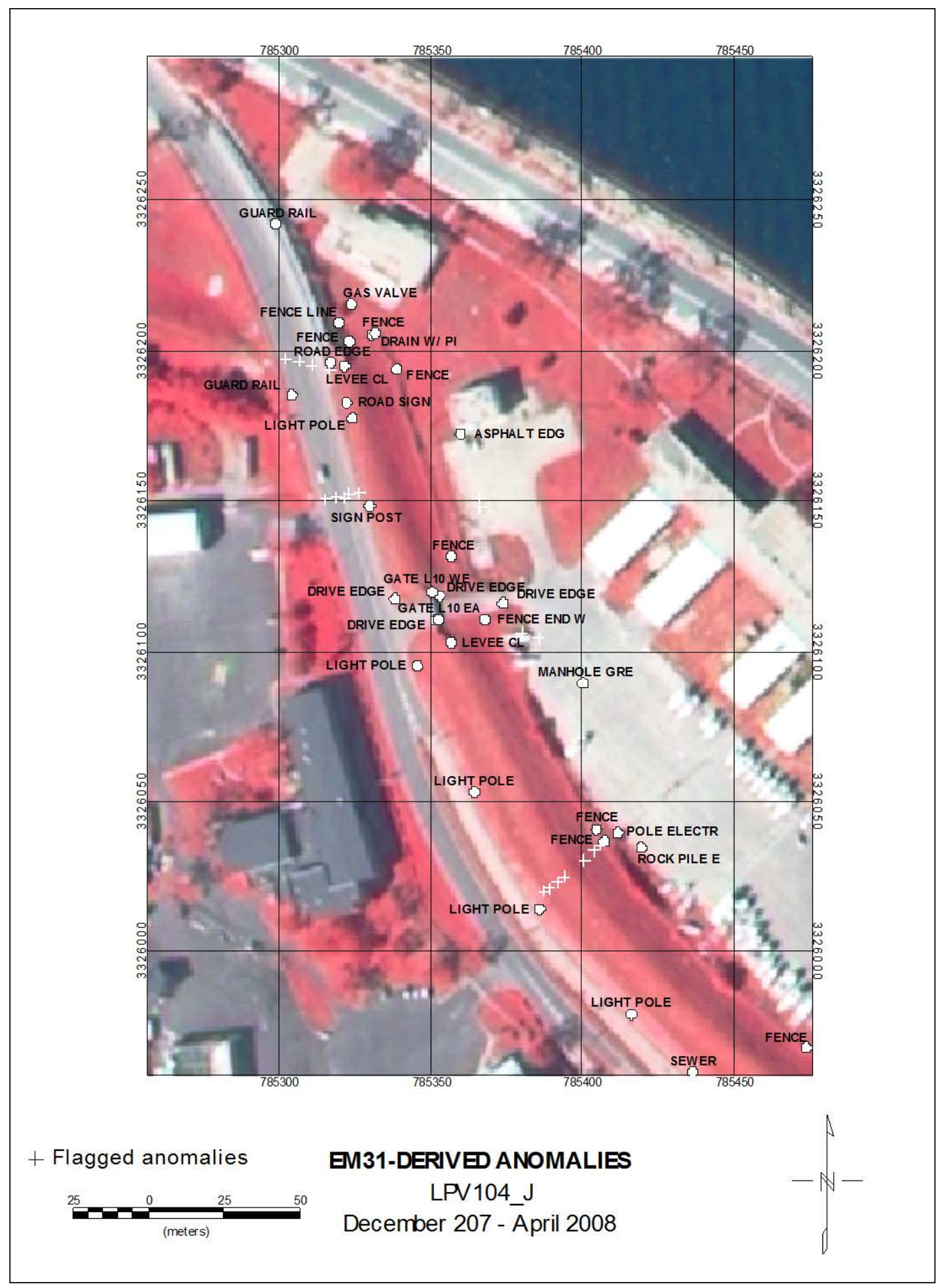




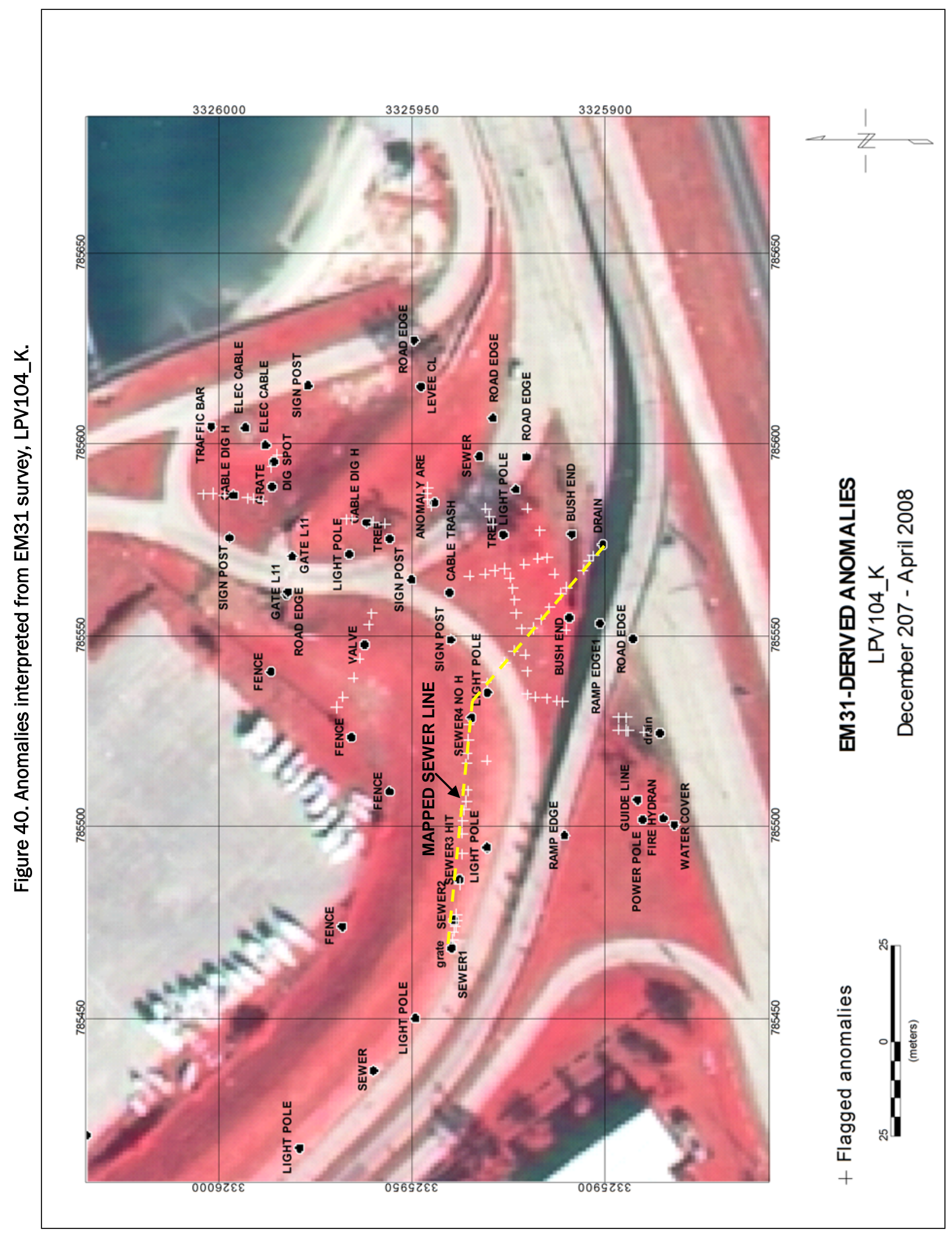


Figure 41. Excavating an EM31 anomaly marked with yellow flags, LPV104_K. UTM Coordinate $(785579.6,3325961.8)$.

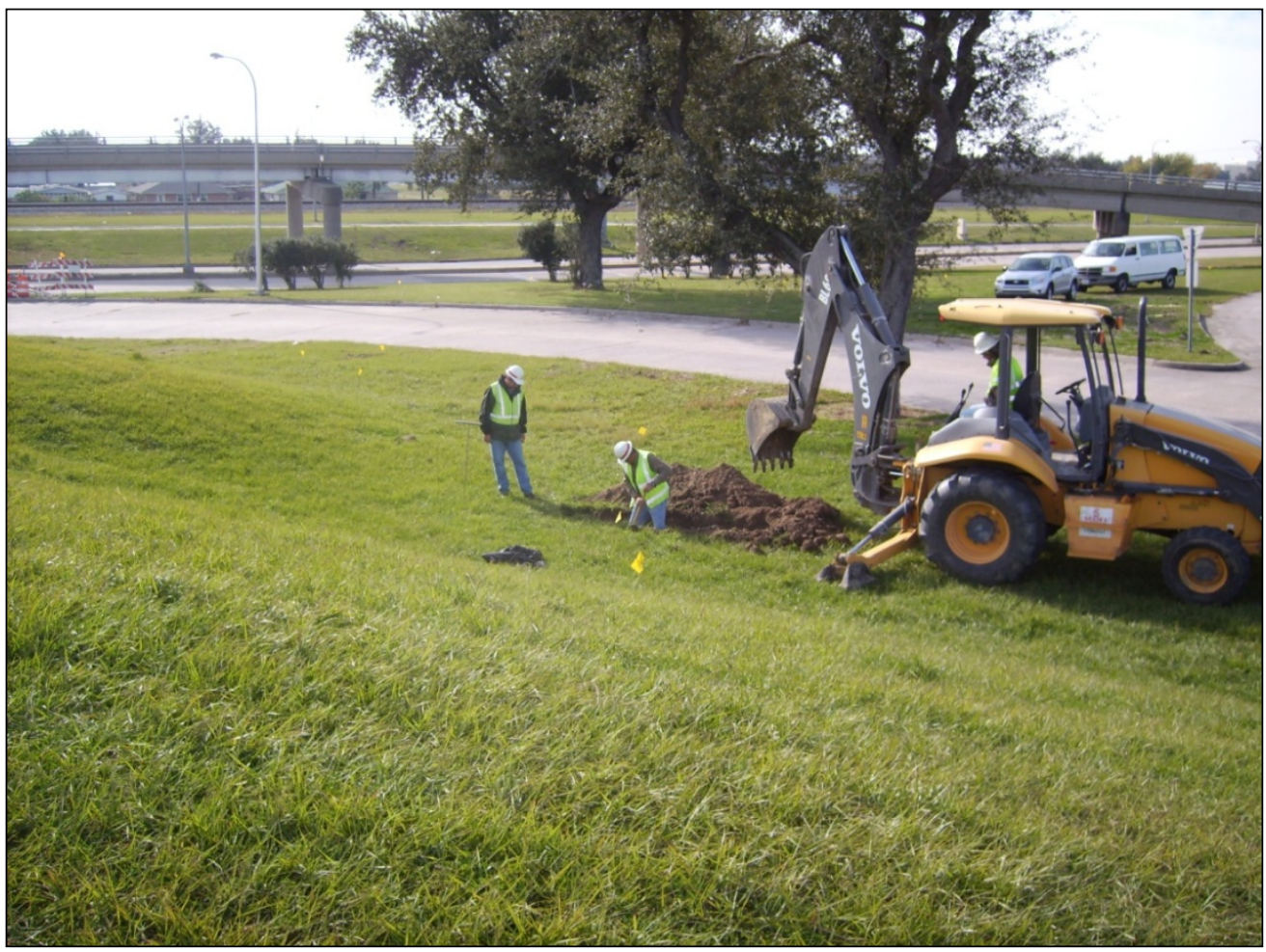

Figure 42. Cable located with the EM31 at bottom of excavation, LPV104_K. UTM Coordinate $(785579.6,3325961.8)$.

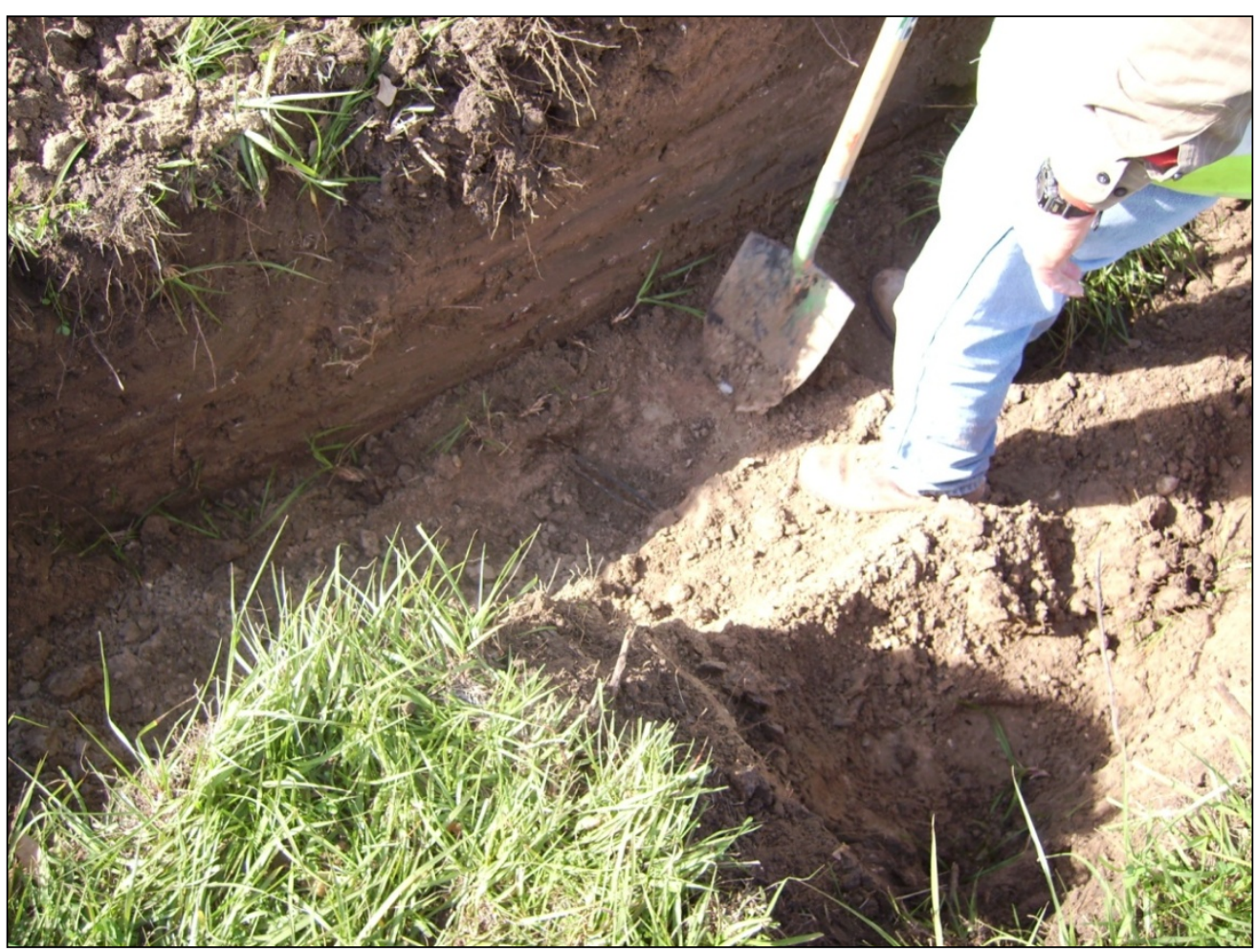


It was decided to use the Radiodetection RD4000 cable and pipe locator system to track the sewer pipe. A radio transmitter was attached to the end of a flexible fiberglass rod, placed into the storm sewer, and pushed at 1- to 2-m intervals (Figure 43). An operator on the ground surface used a radio receiver to track the position of the radio transmitter as it was pushed through the sewer. The locations were marked with flags, thus indicating the alignment of the sewer line (Figure 44). The radio transmitter could

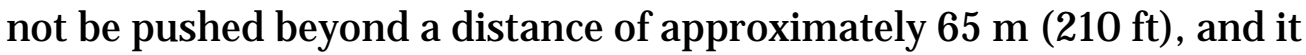
was presumed that it had encountered a bend that it could not negotiate.

Since the transmitter would not go any farther through the sewer line, it was decided to feed it into a drain located at the north edge of Leon C. Simon Drive at UTM coordinate (785524.6, 3325885). This drain had a pipe feeding into it that appeared to be coming from a northwesterly direction and could possibly be connected to the sewer line being investigated. The transmitter was pushed through the pipe to approximately $50 \mathrm{~m}$ ( $165 \mathrm{ft}$ ) or refusal, and its path was mapped. The point of refusal corresponded with that of the previous sewer line. It is presumed that the two lines are connected and that the refusal point is caused by a bend in the line. The mapped sewer line is shown in Figure 40.

Figure 43. Radio transmitter placed in storm sewer, UTM coordinates (785524.6, 3325885.6), LPV104_K.

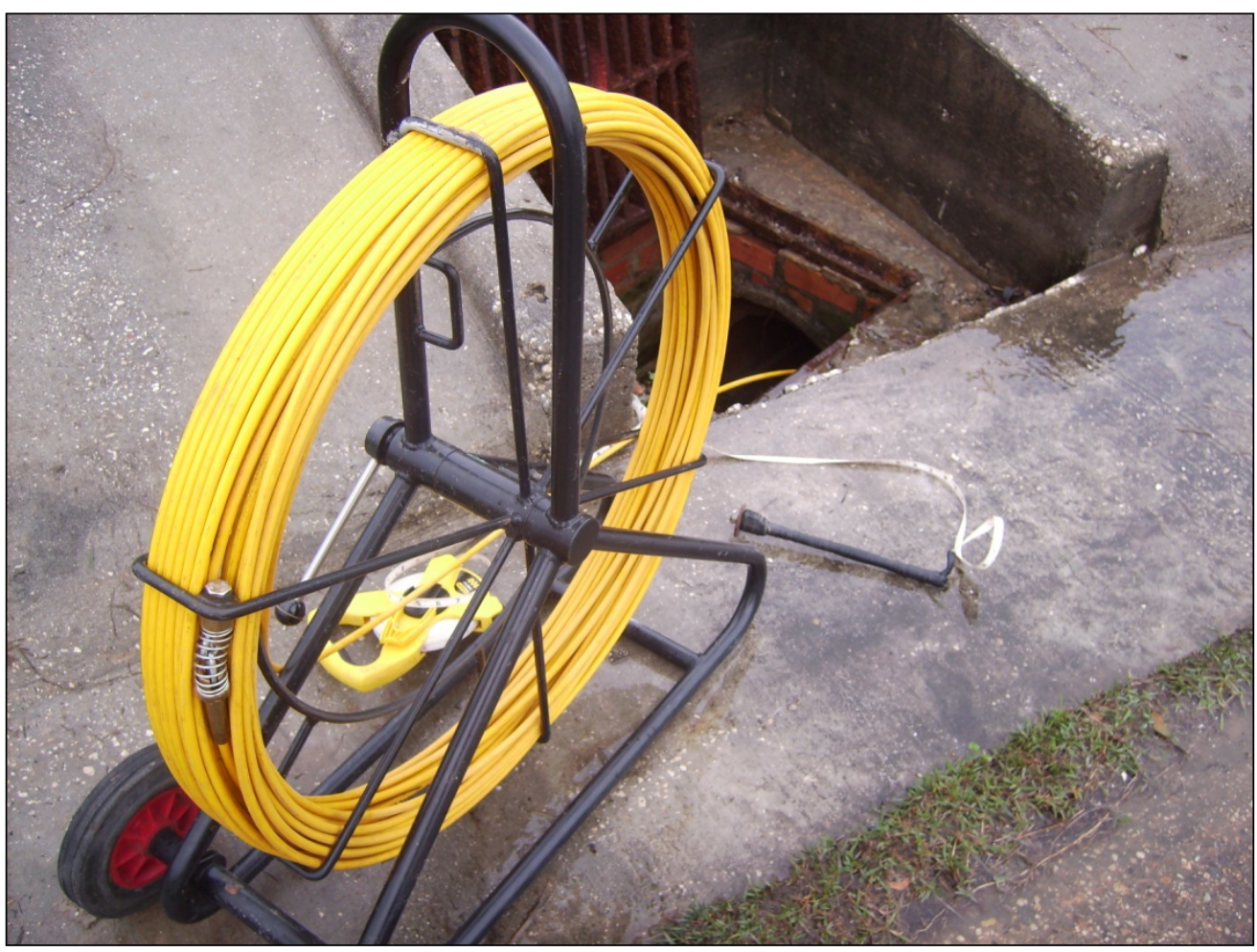


Figure 44. Radio transmitter in storm sewer being tracked on the ground surface. Yellow flags indicate storm sewer track.

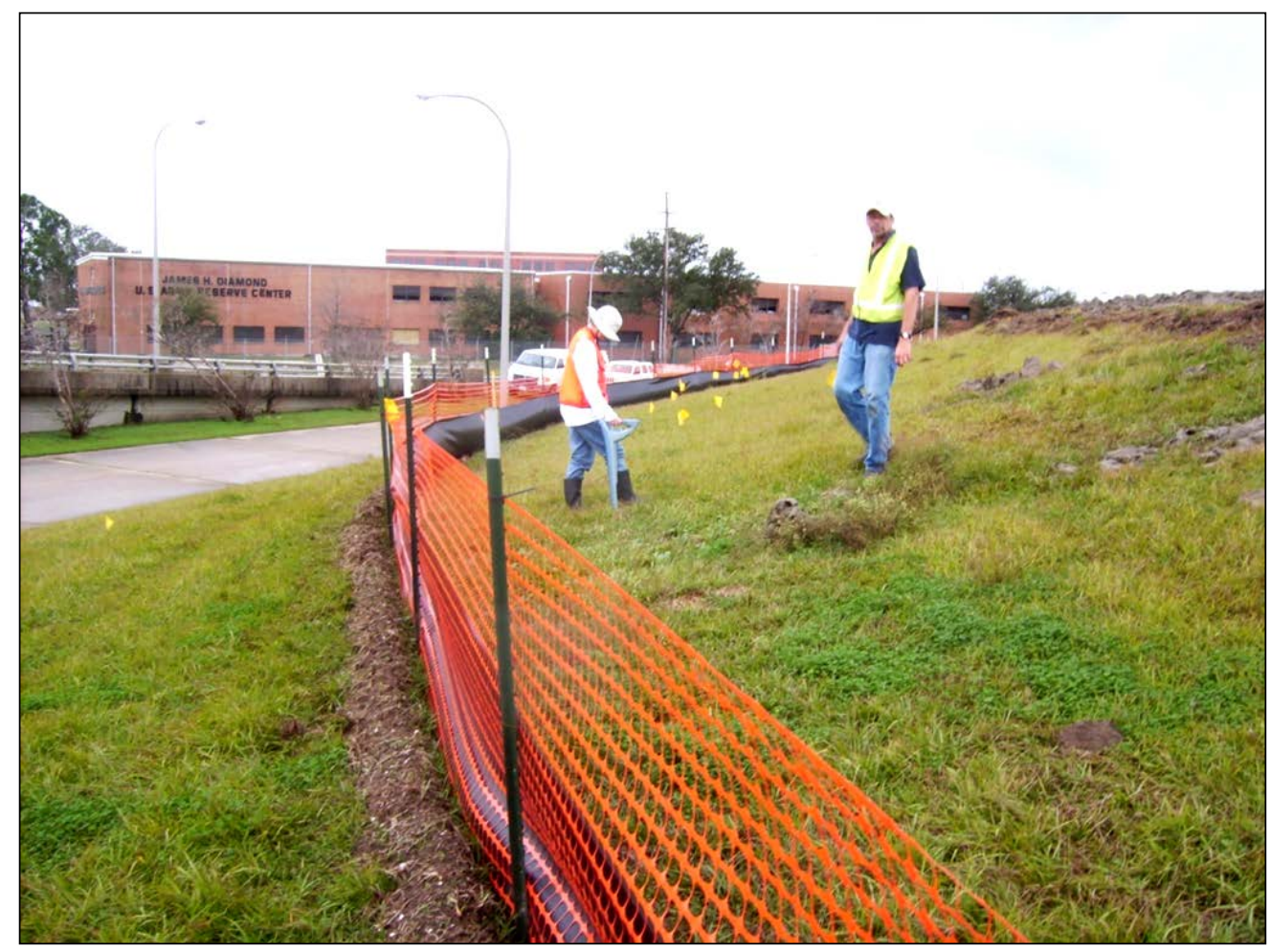

\section{LPV106}

In February 2008, HPO personnel requested that ERDC investigate the location of six seepage areas located along Hayne Boulevard in LPV106 (Figure 45). It was suspected that wells located in the toe of the levee were the source of the seepage. Figure 46 shows one of the seepage sites near the intersection of Hayne Boulevard and Pebble Drive. A magnetometer and an EM61 were used to determine the location of metallic objects, such as well covers or pipes, associated with the seepage site. The area of peak metallic response within each seepage area was marked with a white pin flag and spray paint. The coordinates of the anomalous areas at each seepage location are presented in Table 1 . The anomaly locations, as referenced to the two nearest expansion joints and road curb (Figure 47), are presented in Table 2 .

No signatures indicative of a buried metallic object were observed at the floodgate site, and a very weak metallic signature was indicated at the 14804 Hayne site. 


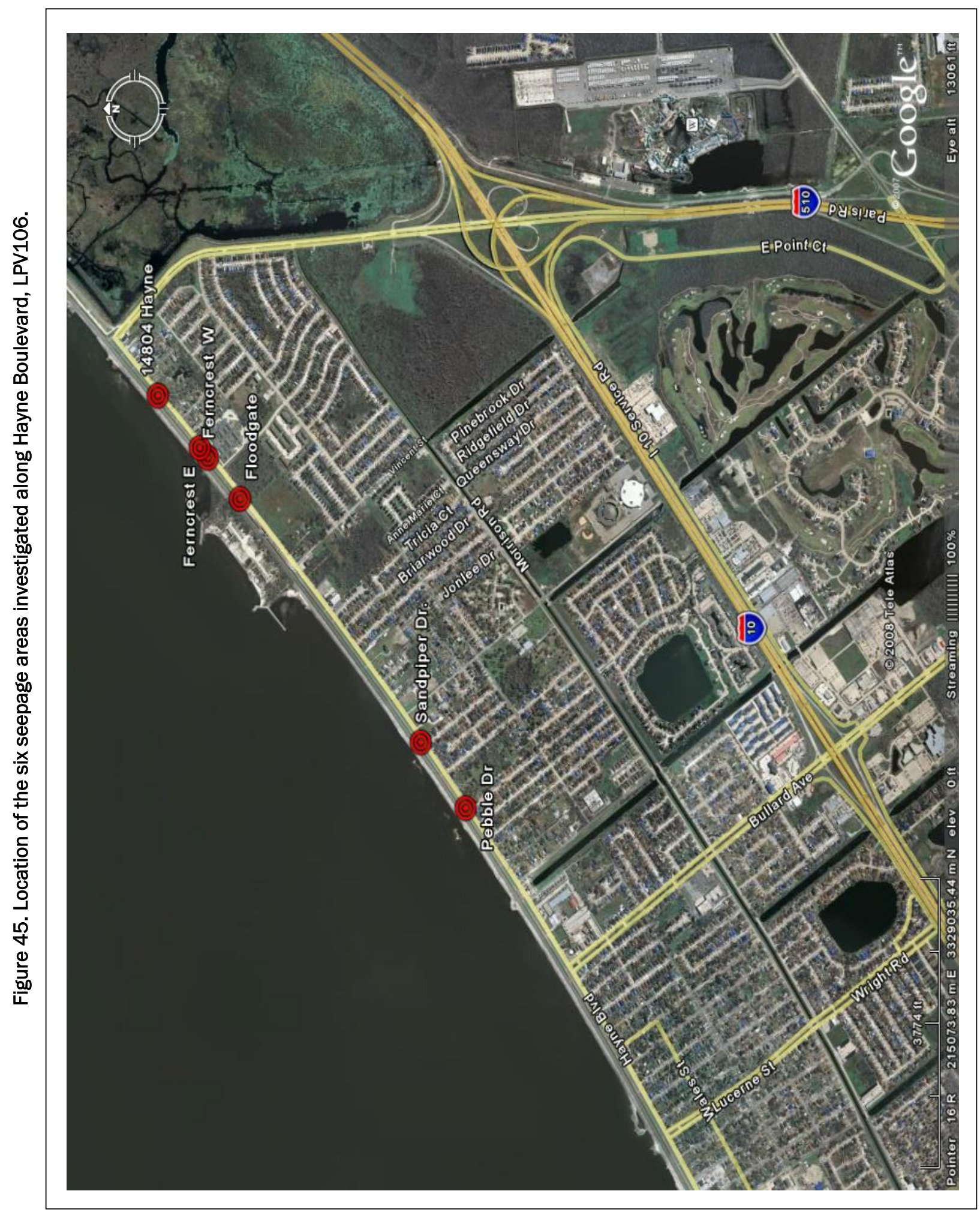


Figure 46. Seepage area, indicated by the white flag on the toe of the levee, located near the intersection of Hayne Boulevard and Pebble Drive.

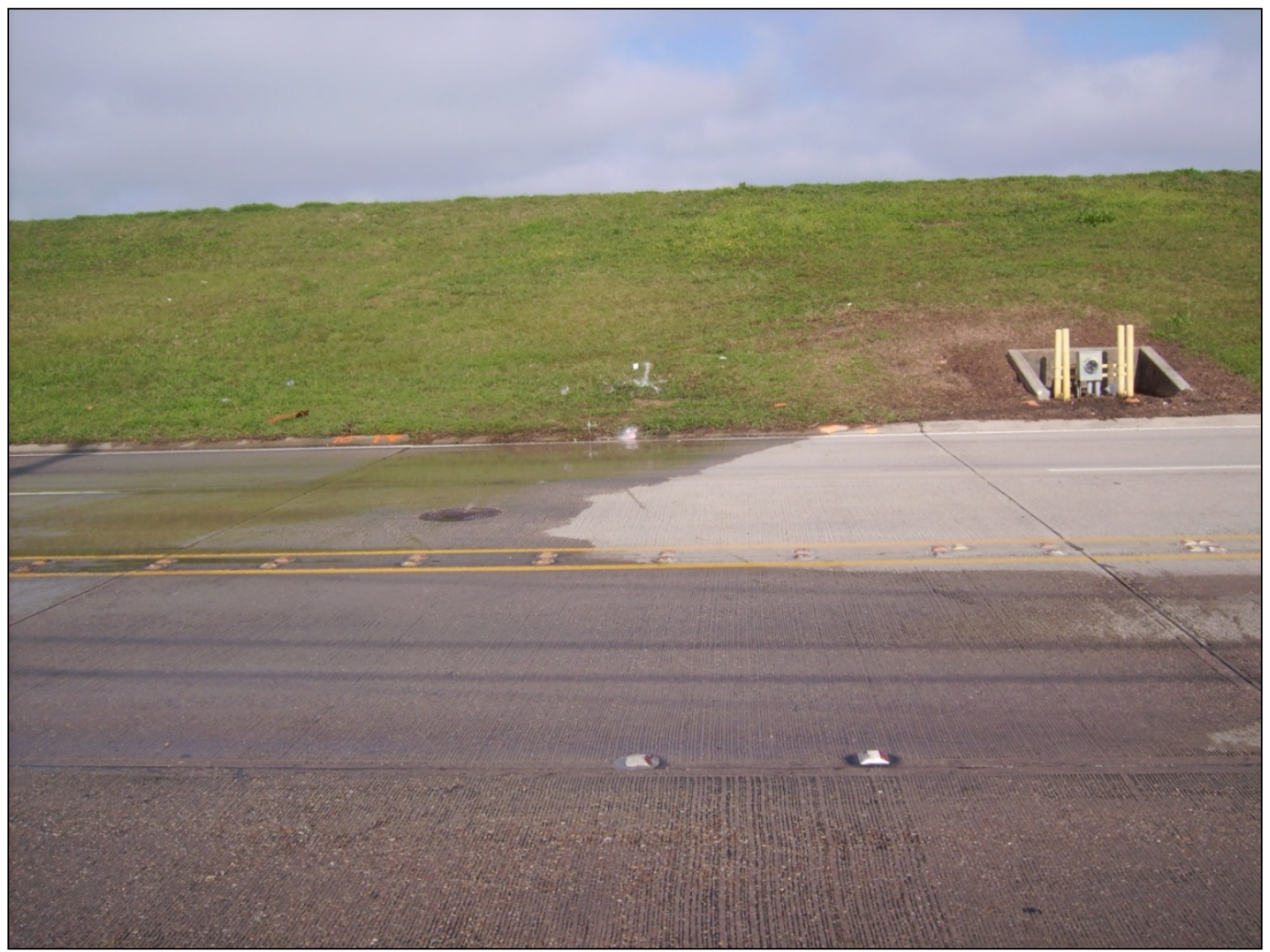

Figure 47. Key for Table 2.

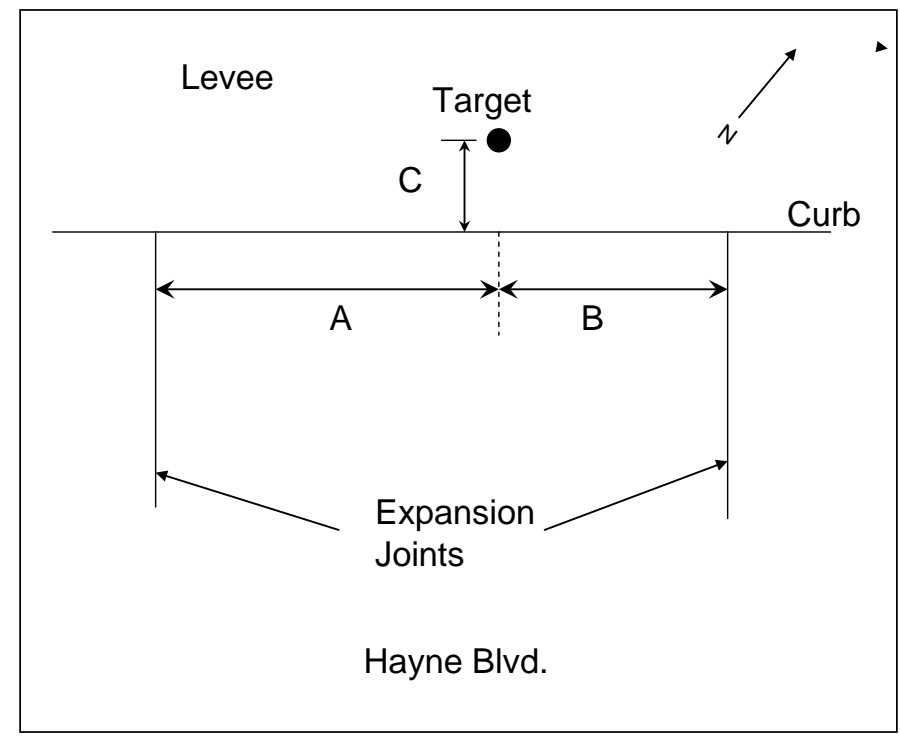


Table 1. Coordinates of seepage locations along Hayne Boulevard, LPV-106.

\begin{tabular}{|l|l|l|l|l|l|l|}
\hline \multirow{2}{*}{ Site } & \multicolumn{2}{|c|}{ UTM (Zone 16) } & \multicolumn{2}{c|}{ Geographic } & \multicolumn{2}{c|}{ State Plane, LA South } \\
\cline { 2 - 7 } & $\mathrm{X}, \mathrm{m}$ & Y, $\mathrm{m}$ & Longitude & Latitude & X, U.S. ft & Y, U.S. ft \\
\hline Pebble Dr. & 214295.6 & 3329268.2 & 895748.34839 & 300340.26061 & 3714175.1 & 570332.9 \\
\hline Sandpiper Dr. & 214562.7 & 3329463.5 & 895738.57461 & 300346.82169 & 3715025.9 & 571005.9 \\
\hline Floodgate & 215552 & 3330253 & 895702.43449 & 300413.26725 & 3718169.1 & 573715.4 \\
\hline Ferncrest West & 215719.4 & 3330393.5 & 895656.32531 & 300417.96602 & 3718700.1 & 574196.5 \\
\hline Ferncrest East & 215759.5 & 3330428.9 & 895654.86354 & 300419.14815 & 3718827.1 & 574317.4 \\
\hline 14804 Hayne Blvd & 215972.5 & 3330612.9 & 895647.09496 & 300425.29636 & 3719502.1 & 574946.7 \\
\hline
\end{tabular}

Note: NAD83.

Table 2. Seepage locations along Hayne Boulevard, LPV-106, referenced to nearest expansion joints and road curb.

\begin{tabular}{|l|l|l|l|}
\hline Location & $\mathrm{A}^{\mathrm{a}}, \mathrm{m}$ & $\mathrm{B}^{\mathrm{a}}, \mathrm{m}$ & $\mathrm{C}, \mathrm{m}$ \\
\hline Pebble Drive & 3.5 & 2.6 & 2.1 \\
\hline Sandpiper Drive & 3.9 & 2.1 & 2.3 \\
\hline Floodgate & $\begin{array}{l}\text { ? (No metallic } \\
\text { signature) }\end{array}$ & $\begin{array}{l}\text { ? (No metallic } \\
\text { signature) }\end{array}$ & $\begin{array}{l}\text { ? (No metallic } \\
\text { signature) }\end{array}$ \\
\hline Ferncrest W & 3.6 & 2.5 & 2.6 \\
\hline Ferncrest E & 4.2 & 1.9 & 2.4 \\
\hline 14804 Hayne Blvd & $\begin{array}{l}3.1 \\
(\text { Weak signal) }\end{array}$ & $\begin{array}{l}3.0 \\
\text { (Weak signal) }\end{array}$ & $\begin{array}{l}1.8 \\
\text { (Weak signal) }\end{array}$ \\
\hline
\end{tabular}

a Note: Refer to Figure 47 for definition of $A, B$, and C. 


\section{Summary and Conclusions}

A geophysical study was performed to determine the locations of anomalies presumed to be associated with buried utilities beneath or in the vicinity of the levees on the south shore of Lake Pontchartrain approximately $8 \mathrm{~km}$ ( 5 miles) north of downtown New Orleans, LA. There was concern that utilities located beneath or buried near the toe of the levees could act as a water conduit during flooding events. If a water-filled utility fails, it is possible that it may cause the levee to fail either by piping material from within the levee or cause slope stability problems. It is also possible that buried utilities can act as potential seepage paths through the levee during high-water events. In this case, the buried pipe would not have to "fail" to cause a problem. The utilities needed to be accurately located so that they can be rerouted, removed, or abandoned and grouted-in.

Electromagnetic, total field magnetic and ground penetrating radar systems were assessed to determine the best method for detecting the buried utilities in this area. The Geonics EM31 electromagnetic induction instrument was considered the most effective for detecting the utilities. EM31 anomalies, presumed to be the locations of buried utilities, were mapped and their coordinates tabulated for further interrogation. 


\section{Appendix A: Tabulated Coordinates of Flagged Anomalies and Utility Locations, LPV101, LPV102, LPV103, and LPV104}

Table A1. LPV101 flagged anomalies and utility locations.

\begin{tabular}{|c|c|c|c|c|c|c|}
\hline \multirow[b]{2}{*}{ Comment } & \multicolumn{2}{|c|}{ UTM (Zone 15) } & \multicolumn{2}{|l|}{ Geographic } & \multicolumn{2}{|c|}{ State Plane, LA South } \\
\hline & $\mathrm{X}, \mathrm{m}$ & $\mathrm{Y}, \mathrm{m}$ & Longitude & Latitude & X, U.S. ft & Y, U.S. ft \\
\hline BLDG & 778411.2 & 3324944.7 & 900647.76227 & 300126.03381 & 3666923.3 & 556238.7 \\
\hline BLDG & 778411.2 & 3324949.0 & 900647.75616 & 300126.17374 & 3666923.7 & 556252.9 \\
\hline BLDG & 778417.4 & 3324944.6 & 900647.52960 & 300126.02594 & 3666943.8 & 556238.1 \\
\hline BLDG & 778418.2 & 3324949.6 & 900647.49552 & 300126.18870 & 3666946.6 & 556254.6 \\
\hline BLDG & 778442.3 & 3324852.3 & 900646.68799 & 300123.00962 & 3667021.0 & 555934.3 \\
\hline BLDG & 778442.4 & 3324847.3 & 900646.69178 & 300122.84918 & 3667020.8 & 555918.0 \\
\hline BLDG & 778444.5 & 3324981.3 & 900646.48742 & 300127.19583 & 3667034.1 & 556357.3 \\
\hline BLDG & 778445.1 & 3324986.3 & 900646.45898 & 300127.35576 & 3667036.5 & 556373.5 \\
\hline BLDG & 778446.8 & 3324847.5 & 900646.52626 & 300122.84964 & 3667035.4 & 555918.2 \\
\hline BLDG & 778447.4 & 3324852.8 & 900646.50013 & 300123.02248 & 3667037.5 & 555935.7 \\
\hline BLDG & 778449.5 & 3324980.8 & 900646.29996 & 300127.17564 & 3667050.6 & 556355.4 \\
\hline BLDG & 778449.5 & 3324985.9 & 900646.29445 & 300127.34101 & 3667050.9 & 556372.1 \\
\hline CABLES & 778427.2 & 3324961.7 & 900647.14762 & 300126.57110 & 3666976.8 & 556293.6 \\
\hline DRAIN & 778403.3 & 3324771.1 & 900648.21975 & 300120.40843 & 3666889.2 & 555670.1 \\
\hline ELEC CONDUI & 778423.8 & 3324715.7 & 900647.50794 & 300118.59396 & 3666953.7 & 555487.5 \\
\hline FLAG & 777829.4 & 3324680.7 & 900709.70409 & 300117.94274 & 3665003.3 & 555400.9 \\
\hline FLAG & 777829.6 & 3324678.5 & 900709.69721 & 300117.87203 & 3665004.0 & 555393.8 \\
\hline FLAG & 777831.1 & 3324675.4 & 900709.64530 & 300117.77070 & 3665008.7 & 555383.6 \\
\hline FLAG & 778409.6 & 3324738.4 & 900648.01474 & 300119.34012 & 3666908.3 & 555562.3 \\
\hline FLAG & 778410.0 & 3324690.4 & 900648.04606 & 300117.78469 & 3666907.2 & 555405.2 \\
\hline FLAG & 778415.2 & 3324986.8 & 900647.57245 & 300127.39715 & 3666938.5 & 556376.6 \\
\hline FLAG & 778415.7 & 3325007.0 & 900647.53375 & 300128.05228 & 3666941.2 & 556442.8 \\
\hline FLAG & 778416.7 & 3324961.7 & 900647.54248 & 300126.58052 & 3666942.1 & 556294.2 \\
\hline FLAG & 778418.8 & 3324986.9 & 900647.43880 & 300127.39864 & 3666950.3 & 556376.9 \\
\hline FLAG & 778419.6 & 3325007.7 & 900647.39015 & 300128.07017 & 3666953.8 & 556444.8 \\
\hline FLAG & 778420.3 & 3324690.2 & 900647.66221 & 300117.76828 & 3666941.0 & 555403.9 \\
\hline FLAG & 778420.7 & 3324695.0 & 900647.64357 & 300117.92320 & 3666942.5 & 555419.6 \\
\hline FLAG & 778421.0 & 3324784.5 & 900647.54728 & 300120.82750 & 3666947.8 & 555713.0 \\
\hline FLAG & 778421.2 & 3324962.0 & 900647.37216 & 300126.58668 & 3666957.0 & 556294.9 \\
\hline FLAG & 778421.3 & 3324701.2 & 900647.61429 & 300118.12622 & 3666944.8 & 555440.1 \\
\hline FLAG & 778422.3 & 3324830.4 & 900647.45572 & 300122.31628 & 3666954.3 & 555863.5 \\
\hline FLAG & 778422.6 & 3324830.5 & 900647.44579 & 300122.31901 & 3666955.1 & 555863.8 \\
\hline
\end{tabular}




\begin{tabular}{|c|c|c|c|c|c|c|}
\hline \multirow[b]{2}{*}{ Comment } & \multicolumn{2}{|c|}{ UTM (Zone 15) } & \multicolumn{2}{|l|}{ Geographic } & \multicolumn{2}{|c|}{ State Plane, LA South } \\
\hline & $\mathrm{X}, \mathrm{m}$ & $\mathrm{Y}, \mathrm{m}$ & Longitude & Latitude & X, U.S. ft & Y, U.S. ft \\
\hline FLAG & 778422.9 & 3325006.4 & 900647.26790 & 300128.02807 & 3666964.6 & 556440.6 \\
\hline FLAG & 778423.3 & 3324909.3 & 900647.34444 & 300124.87645 & 3666961.3 & 556122.2 \\
\hline FLAG & 778424.6 & 3324961.9 & 900647.24537 & 300126.58036 & 3666968.2 & 556294.4 \\
\hline FLAG & 778425.0 & 3324830.2 & 900647.35322 & 300122.30630 & 3666963.3 & 555862.6 \\
\hline FLAG & 778425.2 & 3324830.2 & 900647.34664 & 300122.30680 & 3666963.9 & 555862.6 \\
\hline FLAG & 778426.1 & 3324909.0 & 900647.23948 & 300124.86379 & 3666970.5 & 556121.0 \\
\hline FLAG & 778426.8 & 3324783.9 & 900647.33306 & 300120.80491 & 3666966.7 & 555710.9 \\
\hline FLAG & 778428.2 & 3324830.8 & 900647.23399 & 300122.32498 & 3666973.7 & 555864.6 \\
\hline FLAG & 778428.4 & 3324830.6 & 900647.22825 & 300122.31732 & 3666974.3 & 555863.8 \\
\hline FLAG & 778428.7 & 3324978.9 & 900647.07896 & 300127.12853 & 3666982.2 & 556349.9 \\
\hline FLAG & 778429.2 & 3324961.9 & 900647.07431 & 300126.57735 & 3666983.2 & 556294.3 \\
\hline FLAG & 778429.2 & 3324981.5 & 900647.05515 & 300127.21498 & 3666984.2 & 556358.7 \\
\hline FLAG & 778429.4 & 3324970.9 & 900647.05798 & 300126.87047 & 3666984.3 & 556323.9 \\
\hline FLAG & 778429.6 & 3324909.1 & 900647.10850 & 300124.86315 & 3666982.0 & 556121.1 \\
\hline FLAG & 778429.6 & 3324972.9 & 900647.05036 & 300126.93514 & 3666984.9 & 556330.4 \\
\hline FLAG & 778430.5 & 3324784.8 & 900647.19287 & 300120.83115 & 3666979.0 & 555713.7 \\
\hline FLAG & 778431.3 & 3325027.3 & 900646.93379 & 300128.69941 & 3666993.3 & 556508.8 \\
\hline FLAG & 778431.4 & 3325020.1 & 900646.93700 & 300128.46405 & 3666993.2 & 556485.0 \\
\hline FLAG & 778432.0 & 3324728.0 & 900647.18963 & 300118.98570 & 3666981.2 & 555527.3 \\
\hline FLAG & 778432.0 & 3324983.2 & 900646.94978 & 300127.26783 & 3666993.4 & 556364.1 \\
\hline FLAG & 778432.3 & 3324990.5 & 900646.93061 & 300127.50300 & 3666994.8 & 556387.9 \\
\hline FLAG & 778432.4 & 3324961.7 & 900646.95514 & 300126.56700 & 3666993.7 & 556293.3 \\
\hline FLAG & 778432.5 & 3325011.1 & 900646.90422 & 300128.17135 & 3666996.4 & 556455.4 \\
\hline FLAG & 778432.8 & 3324908.7 & 900646.99171 & 300124.84916 & 3666992.3 & 556119.8 \\
\hline FLAG & 778433.4 & 3325003.0 & 900646.87962 & 300127.90805 & 3666998.9 & 556428.9 \\
\hline FLAG & 778433.8 & 3324831.6 & 900647.02541 & 300122.34488 & 3666992.1 & 555866.8 \\
\hline FLAG & 778433.9 & 3324729.0 & 900647.11959 & 300119.01686 & 3666987.4 & 555530.5 \\
\hline FLAG & 778433.9 & 3324784.3 & 900647.06788 & 300120.81067 & 3666990.0 & 555711.8 \\
\hline FLAG & 778433.9 & 3324831.5 & 900647.02310 & 300122.34340 & 3666992.3 & 555866.6 \\
\hline FLAG & 778434.7 & 3324961.2 & 900646.87011 & 300126.55033 & 3667001.2 & 556291.7 \\
\hline FLAG & 778436.4 & 3324728.0 & 900647.02691 & 300118.98100 & 3666995.5 & 555527.0 \\
\hline FLAG & 778436.9 & 3324732.9 & 900647.00399 & 300119.13968 & 3666997.4 & 555543.0 \\
\hline FLAG & 778438.3 & 3324832.4 & 900646.85615 & 300122.36863 & 3667006.9 & 555869.3 \\
\hline FLAG & 778438.5 & 3324832.2 & 900646.85068 & 300122.36163 & 3667007.4 & 555868.6 \\
\hline FLAG & 778438.5 & 3324908.7 & 900646.77711 & 300124.84363 & 3667011.2 & 556119.4 \\
\hline FLAG & 778438.8 & 3324954.3 & 900646.72296 & 300126.32252 & 3667014.4 & 556268.9 \\
\hline FLAG & 778438.8 & 3324961.0 & 900646.71594 & 300126.54016 & 3667014.7 & 556290.9 \\
\hline FLAG & 778440.8 & 3324784.3 & 900646.80776 & 300120.80399 & 3667012.8 & 555711.3 \\
\hline FLAG & 778441.1 & 3324908.4 & 900646.68225 & 300124.83119 & 3667019.5 & 556118.3 \\
\hline FLAG & 778441.5 & 3324982.9 & 900646.59445 & 300127.25026 & 3667024.7 & 556362.7 \\
\hline
\end{tabular}




\begin{tabular}{|c|c|c|c|c|c|c|}
\hline \multirow[b]{2}{*}{ Comment } & \multicolumn{2}{|c|}{ UTM (Zone 15) } & \multicolumn{2}{|l|}{ Geographic } & \multicolumn{2}{|c|}{ State Plane, LA South } \\
\hline & $\mathrm{X}, \mathrm{m}$ & $\mathrm{Y}, \mathrm{m}$ & Longitude & Latitude & X, U.S. ft & Y, U.S. ft \\
\hline FLAG & 778443.1 & 3324833.0 & 900646.67717 & 300122.38402 & 3667022.6 & 555871.1 \\
\hline FLAG & 778443.2 & 3324954.3 & 900646.56093 & 300126.32078 & 3667028.6 & 556268.8 \\
\hline FLAG & 778443.3 & 3324833.0 & 900646.66992 & 300122.38214 & 3667023.3 & 555870.9 \\
\hline FLAG & 778445.3 & 3324907.6 & 900646.52566 & 300124.80405 & 3667033.3 & 556115.7 \\
\hline FLAG & 778445.6 & 3324833.1 & 900646.58335 & 300122.38472 & 3667030.9 & 555871.2 \\
\hline FLAG & 778445.8 & 3324833.1 & 900646.57751 & 300122.38563 & 3667031.4 & 555871.3 \\
\hline FLAG & 778448.3 & 3324954.2 & 900646.36832 & 300126.31194 & 3667045.6 & 556268.1 \\
\hline FLAG & 778450.4 & 3324987.4 & 900646.25903 & 300127.38900 & 3667054.0 & 556377.0 \\
\hline FLAG & 778450.8 & 3324907.0 & 900646.32181 & 300124.77899 & 3667051.3 & 556113.3 \\
\hline FLAG & 778450.9 & 3324954.2 & 900646.27443 & 300126.31004 & 3667053.8 & 556268.0 \\
\hline FLAG & 778453.3 & 3324954.0 & 900646.18462 & 300126.30099 & 3667061.7 & 556267.2 \\
\hline FLAG & 778453.6 & 3324987.0 & 900646.14285 & 300127.37333 & 3667064.2 & 556375.5 \\
\hline FLAG & 778455.1 & 3324987.2 & 900646.08350 & 300127.37826 & 3667069.4 & 556376.1 \\
\hline HYDRANT & 777827.7 & 3324682.7 & 900709.76559 & 300118.01048 & 3664997.8 & 555407.7 \\
\hline LAMP & 778444.6 & 3324789.5 & 900646.66173 & 300120.96984 & 3667025.5 & 555728.2 \\
\hline LAMP & 778451.1 & 3324869.8 & 900646.34588 & 300123.57065 & 3667050.5 & 555991.2 \\
\hline LAMP BASE & 778401.7 & 3324702.6 & 900648.34413 & 300118.18621 & 3666880.6 & 555445.5 \\
\hline LAMP BASE & 778 & 3324685.2 & 8.30719 & 300117.62150 & 84.5 & 555388.5 \\
\hline MH DRAIN & 778442.8 & 3324732.1 & 900646.78286 & 300119.10970 & 3667016.9 & 555540.2 \\
\hline MH DRAIN & 778443.2 & 3324738.5 & 900646.76291 & 300119.31793 & 3667018.4 & 555561.3 \\
\hline MH DRAIN & 778446.3 & 3324779.9 & 900646.60697 & 300120.65805 & 3667030.7 & 555696.8 \\
\hline MH DRAIN & 778449.2 & 3324821.7 & 900646.45917 & 300122.01339 & 3667042.2 & 555833.8 \\
\hline MH DRAIN & 778456.2 & 3324911.6 & 900646.11647 & 300124.92374 & 3667069.2 & 556128.1 \\
\hline MH NOPSI & 778404.2 & 3324759.1 & 900648.19736 & 300120.01754 & 3666891.5 & 555630.6 \\
\hline MH NOPSI & 778437.3 & 3324703.4 & 900647.01431 & 300118.18415 & 3666997.5 & 555446.5 \\
\hline MH NOPSI & 778440.3 & 3324741.1 & 900646.87004 & 300119.40496 & 3667008.9 & 555570.0 \\
\hline MH NOPSI & 778447.5 & 3324834.4 & 900646.51271 & 300122.42642 & 3667037.0 & 555875.5 \\
\hline MH NOPSI & 778449.5 & 3324850.0 & 900646.42314 & 300122.93030 & 3667044.4 & 555926.5 \\
\hline MH NOPSI & 778453.1 & 3324904.3 & 900646.23571 & 300124.68970 & 3667059.0 & 556104.4 \\
\hline MH SEWER & 777817.4 & 3324694.7 & 900710.13829 & 300118.40871 & 3664964.6 & 555447.6 \\
\hline MH SEWER & 777817.4 & 3324696.0 & 900710.13839 & 300118.45183 & 3664964.6 & 555452.0 \\
\hline MH SEWER & 778400.3 & 3324737.2 & 900648.36389 & 300119.31051 & 3666877.7 & 555559.0 \\
\hline MH SEWER & 778416.8 & 3324736.2 & 900647.74942 & 300119.26426 & 3666931.7 & 555554.9 \\
\hline MH WATER & 777829.5 & 3324679.3 & 900709.69953 & 300117.89777 & 3665003.8 & 555396.4 \\
\hline NOPSI & 778460.1 & 3325005.3 & 900645.88216 & 300127.96141 & 3667086.5 & 556435.2 \\
\hline POWER POLE & 777832.2 & 3324674.3 & 900709.60619 & 300117.73351 & 3665012.1 & 555379.9 \\
\hline POWER POLE & 777836.0 & 3324687.1 & 900709.44969 & 300118.14557 & 3665025.5 & 555421.7 \\
\hline SS INLET & 778439.2 & 3324708.6 & 900646.93877 & 300118.35211 & 3667004.0 & 555463.6 \\
\hline SS INLET & 778441.9 & 3324744.5 & 900646.80405 & 300119.51400 & 3667014.6 & 555581.0 \\
\hline SS INLET & 778445.8 & 3324794.0 & 900646.61220 & 300121.11447 & 3667029.7 & 555742.9 \\
\hline
\end{tabular}




\begin{tabular}{|c|c|c|c|c|c|c|}
\hline \multirow[b]{2}{*}{ Comment } & \multicolumn{2}{|c|}{ UTM (Zone 15) } & \multicolumn{2}{|l|}{ Geographic } & \multicolumn{2}{|c|}{ State Plane, LA South } \\
\hline & $X, m$ & $\mathrm{Y}, \mathrm{m}$ & Longitude & Latitude & X, U.S. ft & Y, U.S. ft \\
\hline SS INLET & 778449.9 & 3324845.2 & 900646.41181 & 300122.77427 & 3667045.5 & 555910.7 \\
\hline SS INLET & 778453.6 & 3324895.4 & 900646.22582 & 300124.40052 & 3667060.1 & 556075.2 \\
\hline VALVE BOX & 778403.7 & 3324700.5 & 900648.27074 & 300118.11577 & 3666887.1 & 555438.4 \\
\hline VAULT & 778416.8 & 3324784.7 & 900647.70357 & 300120.83658 & 3666934.1 & 555713.8 \\
\hline VAULT COX & 778447.5 & 3324832.2 & 900646.51350 & 300122.35556 & 3667037.0 & 555868.3 \\
\hline
\end{tabular}

Note: NAD83.

Table A2. LPV102 flagged anomalies and utility locations.

\begin{tabular}{|c|c|c|c|c|c|c|}
\hline \multirow[b]{2}{*}{ Comment } & \multicolumn{2}{|c|}{ UTM (Zone 15) } & \multicolumn{2}{|c|}{ Geographic } & \multicolumn{2}{|c|}{ State Plane, LA South } \\
\hline & $\mathrm{X}, \mathrm{m}$ & $\mathrm{Y}, \mathrm{m}$ & Longitude & Latitude & X, U.S. ft & Y, U.S. ft \\
\hline BLDG & 778625.2 & 3325211.1 & 900639.53127 & 300134.50301 & 3667637.7 & 557101.9 \\
\hline BLDG & 778627.4 & 3325206.2 & 900639.45269 & 300134.34336 & 3667644.8 & 557085.9 \\
\hline BLDG & 778630.7 & 3325212.8 & 900639.32349 & 300134.55396 & 3667655.9 & 557107.3 \\
\hline BLDG & 778632.3 & 3325208.0 & 900639.26878 & 300134.39617 & 3667660.9 & 557091.4 \\
\hline BLDG & 778791.4 & 3325231.2 & 900633.31352 & 300135.02001 & 3668183.7 & 557160.0 \\
\hline BLDG & 778791.7 & 3325227.5 & 900633.30672 & 300134.89836 & 3668184.4 & 557147.7 \\
\hline BLDG & 778796.4 & 3325232.4 & 900633.12650 & 300135.05281 & 3668200.1 & 557163.5 \\
\hline BLDG & 778797.2 & 3325227.4 & 900633.10332 & 300134.89100 & 3668202.3 & 557147.1 \\
\hline BLDG & 778923.9 & 3325236.3 & 900628.36832 & 300135.07511 & 3668618.3 & 557170.2 \\
\hline BLDG & 778924.3 & 3325241.3 & 900628.34893 & 300135.23644 & 3668619.8 & 557186.5 \\
\hline BLDG & 778929.6 & 3325236.6 & 900628.15828 & 300135.08052 & 3668636.7 & 557170.9 \\
\hline BLDG & 778930.4 & 3325239.7 & 900628.12564 & 300135.17909 & 3668639.5 & 557180.9 \\
\hline BLDG & 780015.6 & 3325271.0 & 900547.63009 & 300135.30582 & 3672198.8 & 557232.0 \\
\hline BLDG & 780016.6 & 3325277.3 & 900547.58579 & 300135.50715 & 3672202.5 & 557252.3 \\
\hline BLDG & 780021.6 & 3325271.1 & 900547.40446 & 300135.30230 & 3672218.6 & 557231.8 \\
\hline BLDG & 780022.2 & 3325276.7 & 900547.37734 & 300135.48511 & 3672220.8 & 557250.3 \\
\hline BLDG & 780024.0 & 3325327.4 & 900547.26489 & 300137.12597 & 3672228.9 & 557416.2 \\
\hline BLDG & 780025.2 & 3325320.5 & 900547.22517 & 300136.90292 & 3672232.6 & 557393.7 \\
\hline BLDG & 780029.4 & 3325328.6 & 900547.06155 & 300137.16262 & 3672246.7 & 557420.1 \\
\hline BLDG & 780030.8 & 3325323.1 & 900547.01453 & 300136.98138 & 3672251.1 & 557401.8 \\
\hline DRAIN & 778428.9 & 3325111.2 & 900646.94693 & 300131.42173 & 3666989.2 & 556783.7 \\
\hline DRAIN & 778437.5 & 3325120.8 & 900646.61442 & 300131.72745 & 3667018.1 & 556814.9 \\
\hline DRAIN & 778467.7 & 3325147.8 & 900645.46417 & 300132.57807 & 3667118.3 & 556901.9 \\
\hline DRAIN & 778471.0 & 3325152.5 & 900645.33797 & 300132.72890 & 3667129.2 & 556917.3 \\
\hline DRAIN & 778574.9 & 3325217.2 & 900641.40053 & 300134.74171 & 3667473.1 & 557124.3 \\
\hline DRAIN & 778646.7 & 3325243.3 & 900638.70027 & 300135.52981 & 3667709.6 & 557206.4 \\
\hline DRAIN & 779722.1 & 3325108.1 & 900558.72796 & 300130.26137 & 3671228.8 & 556711.9 \\
\hline DRAIN & 779755.7 & 3325137.9 & 900557.44741 & 300131.19844 & 3671340.4 & 556807.8 \\
\hline DRAIN & 779791.1 & 3325168.1 & 900556.09970 & 300132.14916 & 3671457.8 & 556905.1 \\
\hline
\end{tabular}




\begin{tabular}{|c|c|c|c|c|c|c|}
\hline \multirow[b]{2}{*}{ Comment } & \multicolumn{2}{|c|}{ UTM (Zone 15) } & \multicolumn{2}{|c|}{ Geographic } & \multicolumn{2}{|c|}{ State Plane, LA South } \\
\hline & $X, m$ & $\mathrm{Y}, \mathrm{m}$ & Longitude & Latitude & X, U.S. ft & Y, U.S. ft \\
\hline DRAIN & 779811.5 & 3325191.2 & 900555.31663 & 300132.88308 & 3671525.8 & 556980.0 \\
\hline DRAIN & 779824.7 & 3325211.9 & 900554.80541 & 300133.54537 & 3671570.0 & 557047.3 \\
\hline FLAG & 778428.9 & 3325103.8 & 900646.95124 & 300131.18062 & 3666989.1 & 556759.4 \\
\hline FLAG & 778433.5 & 3325107.6 & 900646.77860 & 300131.30131 & 3667004.1 & 556771.7 \\
\hline FLAG & 778436.9 & 3325110.0 & 900646.64865 & 300131.37722 & 3667015.5 & 556779.5 \\
\hline FLAG & 778580.1 & 3325213.0 & 900641.21169 & 300134.60301 & 3667489.9 & 557110.5 \\
\hline FLAG & 778631.1 & 3325204.4 & 900639.31905 & 300134.27993 & 3667656.6 & 557079.6 \\
\hline FLAG & 778631.7 & 3325203.7 & 900639.29433 & 300134.25880 & 3667658.8 & 557077.5 \\
\hline FLAG & 778633.2 & 3325203.2 & 900639.24163 & 300134.24154 & 3667663.4 & 557075.8 \\
\hline FLAG & 778636.0 & 3325202.5 & 900639.13706 & 300134.21512 & 3667672.7 & 557073.2 \\
\hline FLAG & 778639.0 & 3325201.6 & 900639.02759 & 300134.18171 & 3667682.3 & 557069.9 \\
\hline FLAG & 778644.5 & 3325199.3 & 900638.82436 & 300134.10539 & 3667700.3 & 557062.4 \\
\hline FLAG & 778648.6 & 3325196.6 & 900638.67421 & 300134.01209 & 3667713.6 & 557053.1 \\
\hline FLAG & 778655.0 & 3325194.2 & 900638.43479 & 300133.92917 & 3667734.7 & 557045.0 \\
\hline FLAG & 778661.0 & 3325192.7 & 900638.21382 & 300133.87561 & 3667754.2 & 557039.8 \\
\hline FLAG & 778758.2 & 3325266.1 & 900634.52189 & 300136.17707 & 3668076.2 & 557275.7 \\
\hline FLAG & 778759.2 & 3325262.3 & 900634.48640 & 300136.05271 & 3668079.5 & 557263.2 \\
\hline FLAG & 778759.8 & 3325257.7 & 900634.46862 & 300135.90313 & 3668081.2 & 557248.1 \\
\hline FLAG & 778759.8 & 3325257.8 & 900634.46864 & 300135.90875 & 3668081.2 & 557248.7 \\
\hline FLAG & 778762.3 & 3325244.9 & 900634.38887 & 300135.48703 & 3668088.6 & 557206.1 \\
\hline FLAG & 778762.8 & 3325239.2 & 900634.37252 & 300135.30235 & 3668090.3 & 557187.5 \\
\hline FLAG & 778764.5 & 3325233.4 & 900634.31587 & 300135.11367 & 3668095.5 & 557168.5 \\
\hline FLAG & 778765.4 & 3325230.6 & 900634.28638 & 300135.01912 & 3668098.2 & 557159.0 \\
\hline FLAG & 778766.2 & 3325226.5 & 900634.25802 & 300134.88687 & 3668100.8 & 557145.6 \\
\hline FLAG & 778767.1 & 3325222.6 & 900634.22864 & 300134.75905 & 3668103.5 & 557132.8 \\
\hline FLAG & 778772.8 & 3325246.3 & 900633.99426 & 300135.52433 & 3668123.3 & 557210.3 \\
\hline FLAG & 778774.1 & 3325243.4 & 900633.94888 & 300135.42956 & 3668127.4 & 557200.8 \\
\hline FLAG & 778775.4 & 3325238.8 & 900633.90427 & 300135.27864 & 3668131.5 & 557185.6 \\
\hline FLAG & 778776.9 & 3325234.6 & 900633.85459 & 300135.14200 & 3668136.0 & 557171.8 \\
\hline FLAG & 778777.9 & 3325228.6 & 900633.82031 & 300134.94508 & 3668139.2 & 557151.9 \\
\hline FLAG & 778777.9 & 3325230.8 & 900633.81981 & 300135.01617 & 3668139.2 & 557159.1 \\
\hline FLAG & 778806.3 & 3325235.4 & 900632.75670 & 300135.14396 & 3668232.5 & 557173.0 \\
\hline FLAG & 778807.3 & 3325240.2 & 900632.71396 & 300135.29731 & 3668236.1 & 557188.6 \\
\hline FLAG & 778807.3 & 3325244.9 & 900632.70974 & 300135.45160 & 3668236.3 & 557204.1 \\
\hline FLAG & 778808.0 & 3325249.5 & 900632.67890 & 300135.60031 & 3668238.8 & 557219.2 \\
\hline FLAG & 778808.4 & 3325253.5 & 900632.65925 & 300135.72708 & 3668240.4 & 557232.0 \\
\hline FLAG & 778808.8 & 3325256.8 & 900632.64215 & 300135.83525 & 3668241.8 & 557243.0 \\
\hline FLAG & \begin{tabular}{|l}
778809.4 \\
\end{tabular} & 3325261.1 & 900632.61497 & 300135.97442 & 3668244.0 & 557257.0 \\
\hline FLAG & 778810.2 & 3325272.3 & 900632.57470 & 300136.33711 & 3668247.2 & 557293.7 \\
\hline FLAG & 778810.8 & 3325275.1 & 900632.55163 & 300136.42761 & 3668249.1 & 557302.9 \\
\hline
\end{tabular}




\begin{tabular}{|c|c|c|c|c|c|c|}
\hline \multirow[b]{2}{*}{ Comment } & \multicolumn{2}{|c|}{ UTM (Zone 15) } & \multicolumn{2}{|c|}{ Geographic } & \multicolumn{2}{|c|}{ State Plane, LA South } \\
\hline & $\mathrm{X}, \mathrm{m}$ & $\mathrm{Y}, \mathrm{m}$ & Longitude & Latitude & X, U.S. ft & Y, U.S. ft \\
\hline FLAG & 778811.0 & 3325280.9 & 900632.53918 & 300136.61471 & 3668250.0 & 557321.8 \\
\hline FLAG & 778819.3 & 3325289.8 & 900632.21968 & 300136.89593 & 3668277.8 & 557350.5 \\
\hline FLAG & 778941.2 & 3325236.9 & 900627.72255 & 300135.07931 & 3668675.0 & 557171.2 \\
\hline FLAG & 778946.2 & 3325236.9 & 900627.53797 & 300135.07804 & 3668691.3 & 557171.3 \\
\hline FLAG & 778951.9 & 3325236.3 & 900627.32462 & 300135.05348 & 3668710.0 & 557169.0 \\
\hline FLAG & 778957.6 & 3325235.9 & 900627.11357 & 300135.03348 & 3668728.6 & 557167.2 \\
\hline FLAG & 778963.1 & 3325236.2 & 900626.90882 & 300135.03907 & 3668746.6 & 557167.9 \\
\hline FLAG & 778968.8 & 3325236.4 & 900626.69483 & 300135.04070 & 3668765.4 & 557168.3 \\
\hline FLAG & 778974.9 & 3325236.3 & 900626.46722 & 300135.03312 & 3668785.4 & 557167.7 \\
\hline FLAG & 778982.7 & 3325224.2 & 900626.18708 & 300134.63546 & 3668810.5 & 557127.8 \\
\hline FLAG & 778982.9 & 3325227.5 & 900626.17751 & 300134.74253 & 3668811.2 & 557138.7 \\
\hline FLAG & 778983.4 & 3325231.1 & 900626.15576 & 300134.85685 & 3668813.0 & 557150.2 \\
\hline FLAG & 779191.3 & 3325221.5 & 900618.41468 & 300134.37646 & 3669493.9 & 557109.0 \\
\hline FLAG & 779201.8 & 3325219.5 & 900618.02320 & 300134.30216 & 3669528.4 & 557101.9 \\
\hline FLAG & 779207.7 & 3325219.2 & 900617.80207 & 300134.28875 & 3669547.9 & 557100.7 \\
\hline FLAG & 779217.0 & 3325219.6 & 900617.45721 & 300134.29207 & 3669578.2 & 557101.4 \\
\hline FLAG & 779219.8 & 3325218.9 & 900617.35213 & 300134.26813 & 3669587.4 & 557099.0 \\
\hline FLAG & 779226.7 & 3325218.8 & 7.09537 & 300 & 10.0 & 557098.3 \\
\hline FLAG & \begin{tabular}{|l}
779232.2 \\
\end{tabular} & 3325218.9 & 900616.89148 & 300134.25760 & 3669627.9 & 557098.4 \\
\hline FLAG & 779236.6 & 3325217.5 & 900616.72641 & 300134.20795 & 3669642.5 & 557093.6 \\
\hline FLAG & 779251.8 & 3325216.9 & 900616.16210 & 300134.17582 & 3669692.1 & 557090.8 \\
\hline FLAG & 779269.0 & 3325216.1 & 900615.52178 & 300134.13548 & 3669748.5 & 557087.4 \\
\hline FLAG & 779282.4 & 3325215.2 & 900615.02151 & 300134.09528 & 3669792.5 & 557083.8 \\
\hline FLAG & 779422.6 & 3325243.7 & 900609.76875 & 300134.90484 & 3670253.3 & 557170.5 \\
\hline FLAG & 779431.2 & 3325247.4 & 900609.44368 & 300135.01794 & 3670281.8 & 557182.2 \\
\hline FLAG & 779431.4 & 3325247.2 & 900609.43487 & 300135.01151 & 3670282.5 & 557181.6 \\
\hline FLAG & \begin{tabular}{|l|}
779437.2 \\
\end{tabular} & 3325250.2 & 900609.21581 & 300135.10389 & 3670301.7 & 557191.1 \\
\hline FLAG & 779437.5 & 3325249.9 & 900609.20623 & 300135.09472 & 3670302.5 & 557190.2 \\
\hline FLAG & 779453.5 & 3325256.7 & 900608.60152 & 300135.30357 & 3670355.5 & 557211.9 \\
\hline FLAG & 779453.5 & 3325257.0 & 900608.60432 & 300135.31243 & 3670355.2 & 557212.8 \\
\hline FLAG & 779459.0 & 3325258.8 & 900608.39729 & 300135.36601 & 3670373.4 & 557218.4 \\
\hline FLAG & 779459.1 & 3325258.6 & 900608.39156 & 300135.36017 & 3670373.9 & 557217.8 \\
\hline FLAG & 779465.1 & 3325261.3 & 900608.16660 & 300135.44378 & 3670393.5 & 557226.5 \\
\hline FLAG & 779465.1 & 3325261.3 & 900608.16508 & 300135.44197 & 3670393.7 & 557226.3 \\
\hline FLAG & 779470.8 & 3325263.6 & 900607.95001 & 300135.51148 & 3670412.5 & 557233.5 \\
\hline FLAG & 779471.0 & 3325263.3 & 900607.94555 & 300135.50330 & 3670412.9 & 557232.7 \\
\hline FLAG & 779474.0 & 3325266.1 & 900607.82989 & 300135.59136 & 3670423.0 & 557241.7 \\
\hline FLAG & \begin{tabular}{|l|}
779474.1 \\
\end{tabular} & 3325265.7 & 900607.82464 & 300135.57690 & 3670423.5 & 557240.2 \\
\hline FLAG & 779476.8 & 3325267.5 & 900607.72339 & 300135.63535 & 3670432.3 & 557246.2 \\
\hline FLAG & 779477.0 & 3325266.9 & 900607.71801 & 300135.61393 & 3670432.8 & 557244.1 \\
\hline
\end{tabular}




\begin{tabular}{|c|c|c|c|c|c|c|}
\hline \multirow[b]{2}{*}{ Comment } & \multicolumn{2}{|c|}{ UTM (Zone 15) } & \multicolumn{2}{|c|}{ Geographic } & \multicolumn{2}{|c|}{ State Plane, LA South } \\
\hline & $X, m$ & $\mathrm{Y}, \mathrm{m}$ & Longitude & Latitude & X, U.S. ft & Y, U.S. ft \\
\hline FLAG & 779479.8 & 3325268.2 & 900607.61112 & 300135.65317 & 3670442.1 & 557248.1 \\
\hline FLAG & 779479.8 & 3325269.0 & 900607.61003 & 300135.67908 & 3670442.2 & 557250.8 \\
\hline FLAG & 779483.2 & 3325271.0 & 900607.48164 & 300135.74193 & 3670453.4 & 557257.2 \\
\hline FLAG & 779483.6 & 3325270.3 & 900607.46778 & 300135.71955 & 3670454.7 & 557255.0 \\
\hline FLAG & 779488.9 & 3325274.5 & 900607.26765 & 300135.85131 & 3670472.1 & 557268.5 \\
\hline FLAG & 779489.1 & 3325274.3 & 900607.26022 & 300135.84481 & 3670472.8 & 557267.8 \\
\hline FLAG & 779522.5 & 3325296.4 & 900605.99398 & 300136.53262 & 3670583.3 & 557338.5 \\
\hline FLAG & 779525.8 & 3325300.0 & 900605.86469 & 300136.64671 & 3670594.6 & 557350.2 \\
\hline FLAG & 779528.9 & 3325301.6 & 900605.74745 & 300136.69832 & 3670604.8 & 557355.5 \\
\hline FLAG & 779532.6 & 3325306.9 & 900605.60615 & 300136.86491 & 3670617.1 & 557372.4 \\
\hline FLAG & 779534.3 & 3325308.8 & 900605.54003 & 300136.92654 & 3670622.8 & 557378.7 \\
\hline FLAG & 779534.9 & 3325309.9 & 900605.51718 & 300136.96292 & 3670624.8 & 557382.4 \\
\hline FLAG & 779536.2 & 3325314.7 & 900605.46300 & 300137.11786 & 3670629.4 & 557398.1 \\
\hline FLAG & 779536.3 & 3325314.7 & 900605.46148 & 300137.11523 & 3670629.5 & 557397.9 \\
\hline FLAG & 779539.7 & 3325320.8 & 900605.32957 & 300137.31294 & 3670640.9 & 557418.0 \\
\hline FLAG & 779615.3 & 3325290.0 & 900602.53634 & 300136.25115 & 3670887.6 & 557313.3 \\
\hline FLAG & 779689.4 & 3325058.0 & 900559.99568 & 300128.66030 & 3671119.1 & 556549.0 \\
\hline FLAG & 779690.3 & 3325065.1 & 900559.95367 & 300128.88964 & 3671122.6 & 556572.2 \\
\hline FLAG & 779691.4 & 3325071.6 & 900559.90670 & 300129.10087 & 3671126.5 & 556593.6 \\
\hline FLAG & 779692.3 & 3325075.8 & 900559.87031 & 300129.23695 & 3671129.5 & 556607.3 \\
\hline FLAG & 779797.5 & 3325317.1 & 900555.71944 & 300136.98038 & 3671485.9 & 557393.5 \\
\hline FLAG & 779798.0 & 3325320.8 & 900555.69802 & 300137.09799 & 3671487.7 & 557405.4 \\
\hline FLAG & 779798.1 & 3325324.5 & 900555.69077 & 300137.22026 & 3671488.2 & 557417.7 \\
\hline FLAG & 779799.0 & 3325330.9 & 900555.65122 & 300137.42767 & 3671491.5 & 557438.7 \\
\hline FLAG & |779799.4 & 3325339.8 & 900555.62550 & 300137.71610 & | 3671493.4 & 557467.9 \\
\hline FLAG & 779799.5 & 3325337.1 & 900555.62472 & 300137.62611 & 3671493.6 & 557458.8 \\
\hline FLAG & 779800.0 & 3325342.2 & 900555.60340 & 300137.79051 & 3671495.3 & 557475.4 \\
\hline FLAG & 779800.1 & 3325343.5 & 900555.59613 & 300137.83396 & 3671495.9 & 557479.8 \\
\hline FLAG & 779800.3 & 3325360.6 & 900555.57133 & 300138.39026 & 3671497.4 & 557536.0 \\
\hline FLAG & 779800.6 & 3325363.9 & 900555.55756 & 300138.49411 & 3671498.5 & 557546.5 \\
\hline FLAG & 779801.0 & 3325367.7 & 900555.54153 & 300138.61902 & 3671499.8 & 557559.1 \\
\hline FLAG & 779801.4 & 3325371.3 & 900555.52094 & 300138.73558 & 3671501.5 & 557570.9 \\
\hline FLAG & 779802.5 & 3325385.6 & 900555.46616 & 300139.19778 & 3671505.8 & 557617.7 \\
\hline FLAG & 779824.9 & 3325199.4 & 900554.80741 & 300133.13895 & 3671570.3 & 557006.3 \\
\hline FLAG & 779834.6 & 3325205.0 & 900554.44091 & 300133.31125 & 3671602.3 & 557024.0 \\
\hline FLAG & 779839.9 & 3325209.1 & 900554.24078 & 300133.43901 & 3671619.8 & 557037.1 \\
\hline FLAG & 779848.7 & 3325216.1 & 900553.90555 & 300133.66002 & 3671649.0 & 557059.8 \\
\hline FLAG & 779856.1 & 3325224.1 & 900553.62014 & 300133.91252 & 3671673.8 & 557085.6 \\
\hline FLAG & 779865.5 & 3325233.1 & 900553.26076 & 300134.19923 & 3671705.1 & 557114.9 \\
\hline FLAG & 779871.1 & 3325302.4 & 900552.98742 & 300136.44148 & 3671726.7 & 557341.6 \\
\hline
\end{tabular}




\begin{tabular}{|c|c|c|c|c|c|c|}
\hline \multirow[b]{2}{*}{ Comment } & \multicolumn{2}{|c|}{ UTM (Zone 15) } & \multicolumn{2}{|c|}{ Geographic } & \multicolumn{2}{|c|}{ State Plane, LA South } \\
\hline & $\mathrm{X}, \mathrm{m}$ & $\mathrm{Y}, \mathrm{m}$ & Longitude & Latitude & X, U.S. ft & Y, U.S. ft \\
\hline FLAG & 779873.3 & 3325243.6 & 900552.96148 & 300134.53344 & 3671731.0 & 557148.9 \\
\hline FLAG & 779877.5 & 3325314.7 & 900552.73663 & 300136.83737 & 3671748.3 & 557381.8 \\
\hline FLAG & 779877.8 & 3325316.4 & 900552.72615 & 300136.89103 & 3671749.1 & 557387.3 \\
\hline FLAG & 779877.8 & 3325322.2 & 900552.71864 & 300137.07951 & 3671749.6 & 557406.3 \\
\hline FLAG & 779878.1 & 3325325.5 & 900552.70391 & 300137.18434 & 3671750.8 & 557416.9 \\
\hline FLAG & 779879.2 & 3325252.6 & 900552.73447 & 300134.82024 & 3671750.7 & 557178.1 \\
\hline FLAG & 779879.9 & 3325300.2 & 900552.66282 & 300136.36418 & 3671755.3 & 557334.1 \\
\hline FLAG & 779882.8 & 3325260.2 & 900552.59147 & 300135.06420 & 3671763.0 & 557202.9 \\
\hline FLAG & 779885.9 & 3325298.1 & 900552.44206 & 300136.29191 & 3671774.8 & 557327.0 \\
\hline FLAG & 779886.4 & 3325269.5 & 900552.44812 & 300135.36103 & 3671775.2 & 557233.0 \\
\hline FLAG & 779889.4 & 3325276.8 & 900552.32965 & 300135.59680 & 3671785.4 & 557256.9 \\
\hline FLAG & 779890.7 & 3325369.1 & 900552.19301 & 300138.59037 & 3671794.2 & 557559.4 \\
\hline FLAG & 779891.2 & 3325284.0 & 900552.25677 & 300135.82723 & 3671791.6 & 557280.3 \\
\hline FLAG & 779891.8 & 3325362.2 & 900552.15845 & 300138.36659 & 3671797.4 & 557536.8 \\
\hline FLAG & 779892.7 & 3325358.6 & 900552.12993 & 300138.24690 & 3671800.1 & 557524.8 \\
\hline FLAG & 779892.9 & 3325288.7 & 900552.18768 & 300135.97925 & 3671797.5 & 557295.7 \\
\hline FLAG & 779893.9 & 3325352.2 & 900552.09003 & 300138.03871 & 3671803.8 & 557503.8 \\
\hline FLAG & 779894.7 & 3325348.7 & 900552.06293 & 300137.92393 & 3671806.3 & 557492.2 \\
\hline FLAG & 779895.4 & 3325345.8 & 900552.04242 & 300137.83003 & 3671808.2 & 557482.8 \\
\hline FLAG & 779953.8 & 3325122.4 & 900550.07648 & 300130.53397 & 3671989.0 & 556747.6 \\
\hline FLAG & 779962.6 & 3325135.2 & 900549.73537 & 300130.94124 & 3672018.5 & 556789.1 \\
\hline FLAG & 779967.3 & 3325140.4 & 900549.55584 & 300131.10725 & 3672034.1 & 556806.0 \\
\hline FLAG & 779978.6 & 3325155.1 & 900549.11918 & 300131.57563 & 3672072.0 & 556853.8 \\
\hline FLAG & 779986.5 & 3325164.5 & 900548.81511 & 300131.87320 & 3672098.4 & 556884.1 \\
\hline FLAG & |779995.4 & 3325176.5 & 900548.47309 & 300132.25415 & 3672128.0 & 556922.9 \\
\hline FLAG & 780010.5 & 3325217.5 & 900547.86985 & 300133.57271 & 3672179.6 & 557056.7 \\
\hline FLAG & 780011.9 & 3325207.9 & 900547.82683 & 300133.25926 & 3672183.7 & 557025.1 \\
\hline FLAG & 780012.3 & 3325428.1 & 900547.60558 & 300140.40359 & 3672195.4 & 557746.9 \\
\hline FLAG & 780012.4 & 3325223.8 & 900547.79400 & 300133.77444 & 3672186.1 & 557077.1 \\
\hline FLAG & 780014.1 & 3325232.7 & 900547.72153 & 300134.06271 & 3672192.1 & 557106.3 \\
\hline FLAG & 780015.9 & 3325206.9 & 900547.67786 & 300133.22552 & 3672196.9 & 557021.8 \\
\hline FLAG & 780016.5 & 3325426.1 & 900547.45040 & 300140.33718 & 3672209.1 & 557740.4 \\
\hline FLAG & 780018.5 & 3325248.2 & 900547.54314 & 300134.56183 & 3672207.3 & 557156.9 \\
\hline FLAG & 780020.4 & 3325430.0 & 900547.30102 & 300140.46063 & 3672222.1 & 557753.0 \\
\hline FLAG & 780020.9 & 3325205.6 & 900547.49325 & 300133.17923 & 3672213.1 & 557017.3 \\
\hline FLAG & 780020.9 & 3325424.9 & 900547.28768 & 300140.29241 & 3672223.5 & 557736.0 \\
\hline FLAG & 780021.1 & 3325418.8 & 900547.28543 & 300140.09681 & 3672223.9 & 557716.2 \\
\hline FLAG & 780022.0 & 3325258.7 & 900547.40422 & 300134.89996 & 3672219.1 & 557191.2 \\
\hline FLAG & 780024.0 & 3325424.8 & 900547.17244 & 300140.28652 & 3672233.6 & 557735.5 \\
\hline FLAG & 780024.5 & 3325402.3 & 900547.17207 & 300139.55723 & 3672234.4 & 557661.8 \\
\hline
\end{tabular}




\begin{tabular}{|c|c|c|c|c|c|c|}
\hline \multirow[b]{2}{*}{ Comment } & \multicolumn{2}{|c|}{ UTM (Zone 15) } & \multicolumn{2}{|c|}{ Geographic } & \multicolumn{2}{|c|}{ State Plane, LA South } \\
\hline & $X, m$ & $\mathrm{Y}, \mathrm{m}$ & Longitude & Latitude & X, U.S. ft & Y, U.S. ft \\
\hline FLAG & 780025.5 & 3325423.1 & 900547.11742 & 300140.23109 & 3672238.5 & 557730.0 \\
\hline FLAG & 780025.6 & 3325397.3 & 900547.13758 & 300139.39416 & 3672237.6 & 557645.4 \\
\hline FLAG & 780026.5 & 3325204.6 & 900547.28478 & 300133.14099 & 3672231.5 & 557013.6 \\
\hline FLAG & 780026.6 & 3325389.9 & 900547.10596 & 300139.15174 & 3672240.7 & 557621.0 \\
\hline FLAG & 780026.8 & 3325281.1 & 900547.20217 & 300135.62277 & 3672236.1 & 557264.4 \\
\hline FLAG & 780027.4 & 3325349.9 & 900547.11533 & 300137.85302 & 3672241.3 & 557489.8 \\
\hline FLAG & 780028.0 & 3325272.2 & 900547.16635 & 300135.33380 & 3672239.5 & 557235.2 \\
\hline FLAG & 780028.0 & 3325382.0 & 900547.06237 & 300138.89484 & 3672244.8 & 557595.0 \\
\hline FLAG & 780028.0 & 3325382.4 & 900547.06183 & 300138.90960 & 3672244.8 & 557596.5 \\
\hline FLAG & 780028.7 & 3325290.6 & 900547.12421 & 300135.92926 & 3672242.6 & 557295.4 \\
\hline FLAG & 780029.2 & 3325372.9 & 900547.02702 & 300138.59851 & 3672248.2 & 557565.1 \\
\hline FLAG & 780029.9 & 3325344.1 & 900547.02646 & 300137.66356 & 3672249.3 & 557470.7 \\
\hline FLAG & 780030.2 & 3325347.8 & 900547.01462 & 300137.78275 & 3672250.2 & 557482.8 \\
\hline FLAG & 780030.5 & 3325353.6 & 900546.99484 & 300137.97354 & 3672251.7 & 557502.0 \\
\hline FLAG & 780030.7 & 3325308.0 & 900547.03136 & 300136.49140 & 3672250.1 & 557352.3 \\
\hline FLAG & 780030.8 & 3325360.9 & 900546.97935 & 300138.20781 & 3672252.8 & 557525.7 \\
\hline FLAG & 780031.3 & 3325422.8 & 900546.90246 & 300140.21595 & 3672257.4 & 557728.6 \\
\hline FLAG & 780033.3 & 3325272.9 & 900546.96660 & 300135.35127 & 3672257.1 & 557237.2 \\
\hline FLAG & 780033.6 & 3325325.9 & 900546.90533 & 300137.07046 & 3672260.6 & 557410.9 \\
\hline FLAG & 780036.7 & 3325326.3 & 900546.79112 & 300137.08057 & 3672270.6 & 557412.0 \\
\hline FLAG & 780039.8 & 3325273.5 & 900546.72631 & 300135.36414 & 3672278.2 & 557238.7 \\
\hline FLAG & 780041.2 & 3325327.1 & 900546.62312 & 300137.10189 & 3672285.3 & 557414.3 \\
\hline FLAG & 780045.5 & 3325274.3 & 900546.51226 & 300135.38545 & 3672297.0 & 557241.1 \\
\hline FLAG & 780045.7 & 3325390.4 & 900546.39305 & 300139.15489 & 3672303.3 & 557621.9 \\
\hline FLAG & 780050.1 & 3325328.8 & 900546.29047 & 300137.14971 & 3672314.5 & 557419.5 \\
\hline GRATE & 779992.2 & 3325157.3 & 900548.61067 & 300131.63423 & 3672116.6 & 556860.2 \\
\hline GRATE & 780034.8 & 3325424.2 & 900546.77043 & 300140.25881 & 3672269.0 & 557733.1 \\
\hline LAMP & 778445.8 & 3325119.5 & 900646.30653 & 300131.67715 & 3667045.2 & 556810.1 \\
\hline LAMP & 778479.1 & 3325154.4 & 900645.03307 & 300132.78136 & 3667156.0 & 556922.9 \\
\hline LAMP & 778510.6 & 3325182.0 & 900643.83266 & 300133.65387 & 3667260.5 & 557012.1 \\
\hline LAMP & 778511.1 & 3325182.3 & 900643.81236 & 300133.66046 & 3667262.3 & 557012.8 \\
\hline LAMP & 778546.5 & 3325209.3 & 900642.46761 & 300134.50911 & 3667379.6 & 557099.8 \\
\hline LAMP & 778587.6 & 3325227.1 & 900640.92019 & 300135.05242 & 3667515.0 & 557156.1 \\
\hline LAMP & 778630.1 & 3325242.4 & 900639.31798 & 300135.51277 & 3667655.4 & 557204.1 \\
\hline LAMP & 778679.6 & 3325243.4 & 900637.47147 & 300135.50544 & 3667817.7 & 557205.1 \\
\hline LAMP & 779710.9 & 3325096.5 & 900559.15497 & 300129.89419 & 3671191.7 & 556674.4 \\
\hline LAMP & 779743.2 & 3325124.3 & 900557.92397 & 300130.76853 & 3671298.9 & 556763.9 \\
\hline LAMP & \begin{tabular}{|l}
779777.7 \\
\end{tabular} & 3325153.8 & 900556.61117 & 300131.69807 & 3671413.3 & 556859.0 \\
\hline LAMP & 779798.3 & 3325309.8 & 900555.69676 & 300136.74192 & 3671488.2 & 557369.4 \\
\hline LAMP & 779813.1 & 3325189.2 & 900555.25810 & 300132.81737 & 3671531.0 & 556973.4 \\
\hline
\end{tabular}




\begin{tabular}{|c|c|c|c|c|c|c|}
\hline \multirow[b]{2}{*}{ Comment } & \multicolumn{2}{|c|}{ UTM (Zone 15) } & \multicolumn{2}{|c|}{ Geographic } & \multicolumn{2}{|c|}{ State Plane, LA South } \\
\hline & $\mathrm{X}, \mathrm{m}$ & $\mathrm{Y}, \mathrm{m}$ & Longitude & Latitude & X, U.S. ft & Y, U.S. ft \\
\hline LAMP & 779836.7 & 3325239.7 & 900554.33105 & 300134.43711 & 3671610.7 & 557137.9 \\
\hline LAMP & 780033.0 & 3325436.4 & 900546.82299 & 300140.65566 & 3672263.9 & 557773.1 \\
\hline LAMP & 780039.2 & 3325407.7 & 900546.62064 & 300139.72086 & 3672282.7 & 557678.9 \\
\hline LAMP & 780048.0 & 3325382.4 & 900546.31549 & 300138.89357 & 3672310.4 & 557595.6 \\
\hline $\mathrm{MH}$ & 780053.5 & 3325323.2 & 900546.16662 & 300136.96707 & 3672325.6 & 557401.2 \\
\hline MH DRAIN & 779835.6 & 3325239.4 & 900554.37191 & 300134.42678 & 3671607.2 & 557136.8 \\
\hline MH DRAIN & 779955.3 & 3325107.1 & 900550.03466 & 300130.03490 & 3671993.2 & 556697.3 \\
\hline MH NOPSI & 779531.8 & 3325312.3 & 900605.63098 & 300137.04254 & 3670614.7 & 557390.4 \\
\hline MH NOPSI & 779779.8 & 3325154.7 & 900556.53200 & 300131.72613 & 3671420.2 & 556861.9 \\
\hline MH NOPSI & 779958.9 & 3325130.4 & 900549.87621 & 300130.78822 & 3672006.3 & 556773.5 \\
\hline MH NOPSI & 780008.3 & 3325210.3 & 900547.95994 & 300133.34029 & 3672171.9 & 557033.1 \\
\hline MH NOPSI 3 & 779558.4 & 3325308.0 & 900604.64412 & 300136.88208 & 3670701.6 & 557375.1 \\
\hline MH SEWER & 778762.1 & 3325277.0 & 900634.36619 & 300136.52907 & 3668089.5 & 557311.4 \\
\hline MH SEWER & 778764.3 & 3325270.2 & 900634.28937 & 300136.30540 & 3668096.5 & 557288.9 \\
\hline MH SEWER & 778779.1 & 3325226.9 & 900633.77804 & 300134.88947 & 3668143.0 & 557146.4 \\
\hline MH SEWER & 779687.5 & 3325078.6 & 900600.04522 & 300129.33071 & 3671114.0 & 556616.7 \\
\hline MH WATER & 779797.6 & 3325313.0 & 900555.71858 & 300136.84708 & 3671486.2 & 557380.0 \\
\hline MH WATER & 779800.5 & 3325383.0 & 900555.54574 & 300139.11499 & 3671498.9 & 557609.2 \\
\hline NOPSI & 778664.3 & 3325190.2 & 900638.09356 & 300133.79355 & 3667764.8 & 557031.6 \\
\hline PHONE & 778762.3 & 3325214.2 & 900634.41535 & 300134.49020 & 3668087.4 & 557105.4 \\
\hline PHONE & 778763.6 & 3325218.0 & 900634.36275 & 300134.61466 & 3668091.9 & 557118.1 \\
\hline PHONE & 778766.6 & 3325223.6 & 900634.24740 & 300134.79265 & 3668101.8 & 557136.1 \\
\hline PHONE & 778768.1 & 3325226.9 & 900634.18803 & 300134.89952 & 3668106.9 & 557147.0 \\
\hline PHONE & 778772.4 & 3325229.6 & 900634.02477 & 300134.98110 & 3668121.2 & 557155.4 \\
\hline PHONE & 778773.6 & 3325247.4 & 900633.96238 & 300135.55820 & 3668126.0 & 557213.7 \\
\hline PHONE & 778773.9 & 3325245.7 & 900633.95562 & 300135.50344 & 3668126.7 & 557208.2 \\
\hline PHONE & 778773.9 & 3325252.1 & 900633.94940 & 300135.71097 & 3668127.0 & 557229.2 \\
\hline PHONE & 778774.0 & 3325257.9 & 900633.94027 & 300135.89827 & 3668127.6 & 557248.1 \\
\hline PHONE & 778774.1 & 3325261.5 & 900633.93043 & 300136.01494 & 3668128.4 & 557259.9 \\
\hline PHONE & 778774.8 & 3325241.3 & 900633.92491 & 300135.36082 & 3668129.6 & 557193.8 \\
\hline PHONE & 778775.6 & 3325267.1 & 900633.87248 & 300136.19616 & 3668133.3 & 557278.3 \\
\hline PHONE & 778775.9 & 3325233.7 & 900633.89052 & 300135.11217 & 3668132.8 & 557168.7 \\
\hline PHONE & 778776.1 & 3325235.9 & 900633.88117 & 300135.18518 & 3668133.6 & 557176.1 \\
\hline PHONE & 778777.7 & 3325271.9 & 900633.78676 & 300136.34964 & 3668140.6 & 557293.8 \\
\hline PHONE BOX & 778780.4 & 3325277.3 & 900633.68260 & 300136.52371 & 3668149.6 & 557311.5 \\
\hline SET PLATE & 779249.7 & 3325227.0 & 900616.23201 & 300134.50489 & 3669685.6 & 557124.0 \\
\hline SET PLATE & 779294.2 & 3325234.8 & 900614.56427 & 300134.72332 & 3669832.0 & 557147.7 \\
\hline SET PLATE & 779385.1 & 3325250.5 & 900611.16090 & 300135.15841 & 3670130.7 & 557194.8 \\
\hline SET PLATE & 779429.4 & 3325257.7 & 900609.50003 & 300135.35340 & 3670276.4 & 557216.1 \\
\hline SET PLATE & 779516.4 & 3325263.9 & 900606.24917 & 300135.48379 & 3670562.0 & 557232.3 \\
\hline
\end{tabular}




\begin{tabular}{|c|c|c|c|c|c|c|}
\hline \multirow[b]{2}{*}{ Comment } & \multicolumn{2}{|c|}{ UTM (Zone 15) } & \multicolumn{2}{|c|}{ Geographic } & \multicolumn{2}{|c|}{ State Plane, LA South } \\
\hline & $\mathrm{X}, \mathrm{m}$ & $\mathrm{Y}, \mathrm{m}$ & Longitude & Latitude & X, U.S. ft & Y, U.S. ft \\
\hline SET PLATE & 779593.3 & 3325276.8 & 900603.37054 & 300135.83932 & 3670814.7 & 557271.0 \\
\hline SET PLATE & 779614.5 & 3325291.2 & 900602.56621 & 300136.28911 & 3670884.9 & 557317.1 \\
\hline SET PLATE & 779685.5 & 3325336.4 & 900559.87606 & 300137.69634 & 3671119.8 & 557461.8 \\
\hline SET PLATE & 779725.1 & 3325337.7 & 900558.39822 & 300137.70614 & 3671249.7 & 557464.2 \\
\hline SET PLATE & 779770.7 & 3325334.3 & 900556.70112 & 300137.56035 & 3671399.0 & 557451.1 \\
\hline SET PLATE & 779819.1 & 3325330.9 & 900554.90009 & 300137.40830 & 3671557.5 & 557437.5 \\
\hline SET PLATE & 779860.0 & 3325322.0 & 900553.38402 & 300137.08864 & 3671691.1 & 557406.6 \\
\hline SIGN & 779723.9 & 3325106.8 & 900558.66136 & 300130.21766 & 3671234.7 & 556707.6 \\
\hline SS DRAIN & 779712.9 & 3325099.3 & 900559.07825 & 300129.98283 & 3671198.3 & 556683.4 \\
\hline SS INLET & 779680.7 & 3325071.5 & 900600.30624 & 300129.10540 & 3671091.3 & 556593.6 \\
\hline SS INLET & 779752.5 & 3325133.1 & 900557.57073 & 300131.04694 & 3671329.7 & 556792.4 \\
\hline SS INLET & 779790.4 & 3325165.2 & 900556.12596 & 300132.05600 & 3671455.6 & 556895.6 \\
\hline SS INLET & 779822.9 & 3325206.6 & 900554.87696 & 300133.37315 & 3671563.9 & 557029.9 \\
\hline SS INLET & 779840.2 & 3325252.2 & 900554.18739 & 300134.83822 & 3671622.9 & 557178.5 \\
\hline SS INLET & 779965.9 & 3325106.1 & 900549.64060 & 300129.99438 & 3672027.9 & 556693.5 \\
\hline SS INLET & 780034.7 & 3325426.8 & 900546.76932 & 300140.34418 & 3672269.0 & 557741.7 \\
\hline SS INLET & 780036.0 & 3325206.5 & 900546.93106 & 300133.19506 & 3672262.5 & 557019.4 \\
\hline SS INLET & 780061.6 & 3325322.6 & 900545.86697 & 300136.93912 & 3672352.0 & 557398.6 \\
\hline STORM DRAIN & 778763.3 & 3325214.9 & 900634.37817 & 300134.51406 & 3668090.6 & 557107.9 \\
\hline UTIL PANL & 778440.0 & 3325114.9 & 900646.52724 & 300131.53154 & 3667026.0 & 556795.2 \\
\hline VAULT ELEC & 778905.1 & 3325282.9 & 900629.02804 & 300136.60447 & 3668558.6 & 557324.1 \\
\hline WATER CVR & 778753.7 & 3325283.3 & 900634.67213 & 300136.74040 & 3668062.4 & 557332.5 \\
\hline
\end{tabular}

Note: NAD83.

Table A3. LPV103 flagged anomalies and utility locations

\begin{tabular}{|l|l|l|l|l|l|l|}
\hline \multirow{2}{*}{ Comment } & \multicolumn{2}{|c|}{ UTM (Zone 15) } & \multicolumn{3}{c|}{ Geographic } & \multicolumn{2}{c|}{ State Plane, LA South } \\
\cline { 2 - 7 } & $\mathrm{X}, \mathrm{m}$ & $\mathrm{Y}, \mathrm{m}$ & Longitude & Latitude & X, U.S. ft & Y, U.S. $\mathrm{ft}$ \\
\hline ANOMALY & 781444.5 & 3325319.5 & 900454.30225 & 300135.69908 & 3676885.7 & 557322.6 \\
\hline ANOMALY & 781452.1 & 3325328.4 & 900454.01233 & 300135.98209 & 3676910.9 & 557351.5 \\
\hline ANOMALY & 781457.8 & 3325338.9 & 900453.78801 & 300136.31692 & 3676930.2 & 557385.5 \\
\hline ANOMALY & 781458.8 & 3325348.9 & 900453.74414 & 300136.63993 & 3676933.7 & 557418.2 \\
\hline ANOMALY & 781461.0 & 3325340.9 & 900453.66758 & 300136.37759 & 3676940.8 & 557391.7 \\
\hline ANOMALY & 781462.6 & 3325350.9 & 900453.59808 & 300136.70056 & 3676946.5 & 557424.4 \\
\hline ANOMALY & 781467.1 & 3325339.6 & 900453.44377 & 300136.33083 & 3676960.5 & 557387.2 \\
\hline ANOMALY & 781471.5 & 3325354.2 & 900453.26353 & 300136.80141 & 3676975.8 & 557434.9 \\
\hline ANOMALY & 781473.6 & 3325339.9 & 900453.20041 & 300136.33514 & 3676981.9 & 557387.9 \\
\hline ANOMALY & 781480.2 & 3325354.1 & 900452.94143 & 300136.79211 & 3677004.1 & 557434.3 \\
\hline ANOMALY & 781482.6 & 3325336.3 & 900452.86768 & 300136.21142 & 3677011.2 & 557375.7 \\
\hline ANOMALY & 781486.9 & 3325350.5 & 900452.69391 & 300136.66974 & 3677026.0 & 557422.2 \\
\hline
\end{tabular}




\begin{tabular}{|c|c|c|c|c|c|c|}
\hline \multirow[b]{2}{*}{ Comment } & \multicolumn{2}{|c|}{ UTM (Zone 15) } & \multicolumn{2}{|c|}{ Geographic } & \multicolumn{2}{|c|}{ State Plane, LA South } \\
\hline & $X, m$ & $\mathrm{Y}, \mathrm{m}$ & Longitude & Latitude & X, U.S. $\mathrm{ft}$ & Y, U.S. ft \\
\hline ANOMALY & 781488.6 & 3325337.6 & 900452.64247 & 300136.24818 & 3677031.0 & 557379.6 \\
\hline ANOMALY & 781490.2 & 3325349.9 & 900452.56911 & 300136.64677 & 3677037.0 & 557420.0 \\
\hline ANOMALY & 781500.7 & 3325335.3 & 900452.19232 & 300136.16494 & 3677070.7 & 557371.7 \\
\hline ANOMALY & 781500.8 & 3325345.6 & 900452.17881 & 300136.49856 & 3677071.5 & 557405.4 \\
\hline ANOMALY & 781507.4 & 3325343.3 & 900451.93597 & 300136.41755 & 3677092.9 & 557397.4 \\
\hline ANOMALY & 781508.9 & 3325338.2 & 900451.88333 & 300136.25178 & 3677097.7 & 557380.7 \\
\hline ANOMALY & 781512.2 & 3325340.3 & 900451.76055 & 300136.31762 & 3677108.4 & 557387.5 \\
\hline BARREL & 781510.0 & 3325404.1 & 900451.78013 & 300138.38931 & 3677104.4 & 557596.8 \\
\hline BLDG & 780015.6 & 3325271.0 & 900547.63009 & 300135.30582 & 3672198.8 & 557232. \\
\hline BLDG & 780016.6 & 3325277.3 & 900547.58579 & 300135.50715 & 3672202.5 & 557252.3 \\
\hline BLDG & 780021.6 & 3325271.1 & 900547.40446 & 300135.30230 & 3672218.6 & 557231.8 \\
\hline BLDG & 780022.2 & 3325276.7 & 900547.37734 & 300135.48511 & 3672220.8 & 557250.3 \\
\hline BLDG & 780024.0 & 3325327.4 & 900547.26489 & 300137.12597 & 3672228.9 & 557416.2 \\
\hline BLDG & 780025.2 & 3325320.5 & 900547.22517 & 300136.90292 & 3672232.6 & 557393.7 \\
\hline BLDG & 780029.4 & 3325328.6 & 900547.06155 & 300137.16262 & 3672246.7 & 557420.1 \\
\hline BLDG & 780030.8 & 3325323.1 & 900547.01453 & 300136.98138 & 3672251.1 & 557401.8 \\
\hline BLDG & 780249.7 & 3325474.4 & 900538.70860 & 300141.71191 & 3672976.0 & 557887.5 \\
\hline BLDG & 780250.0 & 3325469.8 & 900538.70127 & 300141.56032 & 3672976.8 & 557872.2 \\
\hline BLDG & 780256.2 & 3325475.4 & 900538.46533 & 300141.73725 & 3672997.3 & 557890.3 \\
\hline BLDG & 780257.3 & 3325470.8 & 900538.42878 & 300141.58820 & 3673000.7 & 557875.3 \\
\hline BLDG & 780551.8 & 3325496.4 & 900527.42099 & 300142.17614 & 3673967.6 & 557945.2 \\
\hline BLDG & 780553.6 & 3325501.6 & 900527.34916 & 300142.34344 & 3673973.7 & 557962.1 \\
\hline BLDG & 780556.7 & 3325494.2 & 900527.24007 & 300142.09934 & 3673983.6 & 557937.6 \\
\hline BLDG & 780558.9 & 3325499.2 & 900527.15378 & 300142.26091 & 3673991.0 & 557954.0 \\
\hline BLDG & 781009.2 & 3325339.0 & 900510.51611 & 300136.69095 & 3675459.5 & 557407.2 \\
\hline BLDG & 781010.7 & 3325345.9 & 900510.45442 & 300136.91477 & 3675464.7 & 557429.9 \\
\hline BLDG & 781014.6 & 3325338.5 & 900510.31475 & 300136.67038 & 3675477.2 & 557405.4 \\
\hline BLDG & 781017.4 & 3325344.1 & 900510.20430 & 300136.85024 & 3675486.7 & 557423.6 \\
\hline BLDG & 781296.7 & 3325087.8 & 900500.03482 & 300128.30209 & 3676390.0 & 556569. \\
\hline BUSH & 782084.8 & 3325768.7 & 900430.00083 & 300149.74251 & 3679006.2 & 558764.5 \\
\hline CENTERLINE & 781877.6 & 3325818.6 & 900437.67731 & 300151.53318 & 3678329.5 & 558938. \\
\hline CENTERLINE & 781889.0 & 3325828.2 & 900437.24427 & 300151.83634 & 3678367.2 & 558969. \\
\hline CENTERLINE & 781929.4 & 3325827.1 & 900435.74079 & 300151.76459 & 3678499.4 & 558963.2 \\
\hline CENTERLINE & 781966.8 & 3325826.8 & 900434.34470 & 300151.72582 & 3678622.2 & 558960.7 \\
\hline CENTERLINE & 782001.0 & 3325827.0 & 900433.06808 & 300151.70391 & 3678734.4 & 558959.7 \\
\hline CENTERLINE & 782014.2 & 3325826.2 & 900432.57972 & 300151.66743 & 3678777.4 & 558956.5 \\
\hline CENTERLINE & 782024.3 & 3325821.5 & 900432.20710 & 300151.50515 & 3678810.3 & 558940. \\
\hline CENTERLINE & 782047.6 & 3325805.3 & 900431.35316 & 300150.96135 & 3678885.9 & 558886.3 \\
\hline CENTERLINE & 782069.7 & 3325788.6 & 900430.54392 & 300150.40017 & 3678957.7 & 558830. \\
\hline CENTERLINE & 782120.5 & 3325769.9 & 900428.66753 & 300149.75122 & 3679123.3 & 558766.7 \\
\hline
\end{tabular}




\begin{tabular}{|c|c|c|c|c|c|c|}
\hline \multirow[b]{2}{*}{ Comment } & \multicolumn{2}{|c|}{ UTM (Zone 15) } & \multicolumn{2}{|c|}{ Geographic } & \multicolumn{2}{|c|}{ State Plane, LA South } \\
\hline & $\mathrm{X}, \mathrm{m}$ & $\mathrm{Y}, \mathrm{m}$ & Longitude & Latitude & X, U.S. ft & Y, U.S. ft \\
\hline CENTERLINE & 782122.0 & 3325802.4 & 900428.57955 & 300150.80458 & 3679129.9 & 558873.2 \\
\hline CENTERLINE & 782124.2 & 3325759.1 & 900428.54103 & 300149.39718 & 3679134.8 & 558731.1 \\
\hline CENTERLINE & 782132.1 & 3325805.2 & 900428.20359 & 300150.88822 & 3679162.9 & 558882.0 \\
\hline CONCRETE & 782097.8 & 3325805.5 & 900429.47944 & 300150.92496 & 3679050.7 & 558884.5 \\
\hline CONCRETE & 782102.4 & 3325782.9 & 900429.32937 & 300150.18731 & 3679064.7 & 558810.1 \\
\hline CONCRETE & 782114.2 & 3325789.9 & 900428.88407 & 300150.40467 & 3679103.6 & 558832.5 \\
\hline CONCRETE & 782117.0 & 3325790.0 & 900428.78027 & 300150.40570 & 3679112.7 & 558832.7 \\
\hline CONCRETE & 782117.4 & 3325765.6 & 900428.78781 & 300149.61478 & 3679112.9 & 558752.8 \\
\hline CONCRETE & 782121.3 & 3325793.1 & 900428.61731 & 300150.50519 & 3679126.9 & 558842.9 \\
\hline CONCRETE & 782121.5 & 3325765.8 & 900428.63597 & 300149.61880 & 3679126.3 & 558753.4 \\
\hline CONCRETE & 782125.7 & 3325812.1 & 900428.43586 & 300151.11563 & 3679142.2 & 558904.7 \\
\hline DRAIN & 779722.1 & 3325108.1 & 900558.72796 & 300130.26137 & 3671228.8 & 556711.9 \\
\hline DRAIN & 779755.7 & 3325137.9 & 900557.44741 & 300131.19844 & 3671340.4 & 556807.8 \\
\hline DRAIN & 779791.1 & 3325168.1 & 900556.09970 & 300132.14916 & 3671457.8 & 556905.1 \\
\hline DRAIN & 779811.5 & 3325191.2 & 900555.31663 & 300132.88308 & 3671525.8 & 556980.0 \\
\hline DRAIN & 779824.7 & 3325211.9 & 900554.80541 & 300133.54537 & 3671570.0 & 557047.3 \\
\hline DRAIN & 781432.7 & 3325212.5 & 900454.84498 & 300132.23466 & 3676841.8 & 556972.1 \\
\hline DRAIN & 781518.1 & 3325335.3 & 900451.54268 & 300136.14856 & 3677127.8 & 557370.6 \\
\hline DRAIN & 781557.5 & 3325447.1 & 900449.96982 & 300139.74361 & 3677262.1 & 557735.3 \\
\hline DRAIN & 781563.3 & 3325339.6 & 900449.85607 & 300136.25361 & 3677275.9 & 557382.9 \\
\hline DRAIN & 781597.1 & 3325344.6 & 900448.58967 & 300136.38617 & 3677387.1 & 557397.5 \\
\hline DRAIN & 781624.6 & 3325356.9 & 900447.55256 & 300136.76102 & 3677477.8 & 557436.3 \\
\hline DRAIN & 781637.8 & 3325365.4 & 900447.05280 & 300137.02725 & 3677521.5 & 557463.7 \\
\hline EL STA & 782052.4 & 3325776.8 & 900431.19912 & 300150.03245 & 3678900.5 & 558792.7 \\
\hline ELEC STATIO & 781765.2 & 3325608.7 & 900442.06855 & 300144.81521 & 3677950.9 & 558255.2 \\
\hline ELECTRIC & 782041.3 & 3325769.0 & 900431.62397 & 300149.78950 & 3678863.4 & 558767.7 \\
\hline ELECTRIC & 782052.5 & 3325775.3 & 900431.19917 & 300149.98365 & 3678900.6 & 558787.7 \\
\hline EXCAVATION & 780247.7 & 3325499.1 & 900538.75873 & 300142.51507 & 3672970.7 & 557968.6 \\
\hline FIBER OPTIC & 781944.8 & 3325775.7 & 900435.21390 & 300150.08523 & 3678547.6 & 558794.1 \\
\hline FLAG & 779689.4 & 3325058.0 & 900559.99568 & 300128.66030 & 3671119.1 & 556549.0 \\
\hline FLAG & 779690.3 & 3325065.1 & 900559.95367 & 300128.88964 & 3671122.6 & 556572.2 \\
\hline FLAG & 779691.4 & 3325071.6 & 900559.90670 & 300129.10087 & 3671126.5 & 556593.6 \\
\hline FLAG & 779692.3 & 3325075.8 & 900559.87031 & 300129.23695 & 3671129.5 & 556607.3 \\
\hline FLAG & 779824.9 & 3325199.4 & 900554.80741 & 300133.13895 & 3671570.3 & 557006.3 \\
\hline FLAG & 779834.6 & 3325205.0 & 900554.44091 & 300133.31125 & 3671602.3 & 557024.0 \\
\hline FLAG & 779839.9 & 3325209.1 & 900554.24078 & 300133.43901 & 3671619.8 & 557037.1 \\
\hline FLAG & 779848.7 & 3325216.1 & 900553.90555 & 300133.66002 & 3671649.0 & 557059.8 \\
\hline FLAG & 779856.1 & 3325224.1 & 900553.62014 & 300133.91252 & 3671673.8 & 557085.6 \\
\hline FLAG & 779865.5 & 3325233.1 & 900553.26076 & 300134.19923 & 3671705.1 & 557114.9 \\
\hline FLAG & 779871.1 & 3325302.4 & 900552.98742 & 300136.44148 & 3671726.7 & 557341.6 \\
\hline
\end{tabular}




\begin{tabular}{|c|c|c|c|c|c|c|}
\hline \multirow[b]{2}{*}{ Comment } & \multicolumn{2}{|c|}{ UTM (Zone 15) } & \multicolumn{2}{|c|}{ Geographic } & \multicolumn{2}{|c|}{ State Plane, LA South } \\
\hline & $\mathrm{X}, \mathrm{m}$ & $\mathrm{Y}, \mathrm{m}$ & Longitude & Latitude & X, U.S. ft & Y, U.S. ft \\
\hline FLAG & 779873.3 & 3325243.6 & 900552.96148 & 300134.53344 & 3671731.0 & 557148.9 \\
\hline FLAG & |779879.2 & 3325252.6 & 900552.73447 & 300134.82024 & 3671750.7 & 557178.1 \\
\hline FLAG & 779879.9 & 3325300.2 & 900552.66282 & 300136.36418 & 3671755.3 & 557334.1 \\
\hline FLAG & 779882.8 & 3325260.2 & 900552.59147 & 300135.06420 & 3671763.0 & 557202. \\
\hline FLAG & 779885.9 & 3325298.1 & 900552.44206 & 300136.29191 & 3671774.8 & 557327.0 \\
\hline FLAG & 779886.4 & 3325269.5 & 900552.44812 & 300135.36103 & 3671775.2 & 557233. \\
\hline FLAG & 779889.4 & 3325276.8 & 900552.32965 & 300135.59680 & 3671785.4 & 557256.9 \\
\hline FLAG & 779891.2 & 3325284.0 & 900552.25677 & 300135.82723 & 3671791.6 & 557280.3 \\
\hline FLAG & 779892.9 & 3325288.7 & 900552.18768 & 300135.97925 & 3671797.5 & 557295.7 \\
\hline FLAG & 779953.8 & 3325122.4 & 900550.07648 & 300130.53397 & 3671989.0 & 556747.6 \\
\hline FLAG & 779962.6 & 3325135.2 & 900549.73537 & 300130.94124 & 3672018.5 & 556789.1 \\
\hline FLAG & 779967.3 & 3325140.4 & 900549.55584 & 300131.10725 & 3672034.1 & 556806. \\
\hline FLAG & 779978.6 & 3325155.1 & 900549.11918 & 300131.57563 & 3672072.0 & 556853.8 \\
\hline FLAG & 779986.5 & 3325164.5 & 900548.81511 & 300131.87320 & | 3672098.4 & 556884.1 \\
\hline FLAG & 779995.4 & 3325176.5 & 900548.47309 & 300132.25415 & 3672128.0 & 556922. \\
\hline FLAG & 780010.5 & 3325217.5 & 900547.86985 & 300133.57271 & 3672179.6 & 557056.7 \\
\hline FLAG & 780011.9 & 3325207.9 & 900547.82683 & 300133.25926 & 3672183.7 & 557025.1 \\
\hline FLAG & 780012.3 & 3325428.1 & 900547.60558 & 300140.40359 & 3672195.4 & 557746.9 \\
\hline FLAG & 780012.4 & 3325223.8 & 900547.79400 & 300133.77444 & 3672186.1 & 557077.1 \\
\hline FLAG & 780014.1 & 3325232.7 & 900547.72153 & 300134.06271 & 3672192.1 & 557106.3 \\
\hline FLAG & 780015.9 & 3325206.9 & 900547.67786 & 300133.22552 & 3672196.9 & 557021.8 \\
\hline FLAG & 780016.5 & 3325426.1 & 900547.45040 & 300140.33718 & 3672209.1 & 557740.4 \\
\hline FLAG & 780018.5 & 3325248.2 & 900547.54314 & 300134.56183 & 3672207.3 & 557156.9 \\
\hline FLAG & 780020.4 & 3325430.0 & 900547.30102 & 300140.46063 & 3672222.1 & 557753.0 \\
\hline FLAG & 780020.9 & 3325424.9 & 900547.28768 & 300140.29241 & 3672223.5 & 557736.0 \\
\hline FLAG & 780020.9 & 3325205.6 & 900547.49325 & 300133.17923 & 3672213.1 & 557017.3 \\
\hline FLAG & 780021.1 & 3325418.8 & 900547.28543 & 300140.09681 & 3672223.9 & 557716.2 \\
\hline FLAG & 780022.0 & 3325258.7 & 900547.40422 & 300134.89996 & 3672219.1 & 557191.2 \\
\hline FLAG & 780024.0 & 3325424.8 & 900547.17244 & 300140.28652 & 3672233.6 & 557735.5 \\
\hline FLAG & 780024.5 & 3325402.3 & 900547.17207 & 300139.55723 & 3672234.4 & 557661.8 \\
\hline FLAG & 780025.5 & 3325423.1 & 900547.11742 & 300140.23109 & 3672238.5 & 557730.0 \\
\hline FLAG & 780025.6 & 3325397.3 & 900547.13758 & 300139.39416 & 3672237.6 & 557645.4 \\
\hline FLAG & 780026.5 & 3325204.6 & 900547.28478 & 300133.14099 & 3672231.5 & 557013.6 \\
\hline FLAG & 780026.6 & 3325389.9 & 900547.10596 & 300139.15174 & 3672240.7 & 557621.0 \\
\hline FLAG & \begin{tabular}{|l|}
780026.8 \\
\end{tabular} & 3325281.1 & 900547.20217 & 300135.62277 & 3672236.1 & 557264. \\
\hline FLAG & 780027.4 & 3325349.9 & 900547.11533 & 300137.85302 & 3672241.3 & 557489.8 \\
\hline FLAG & 780028.0 & 3325272.2 & 900547.16635 & 300135.33380 & 3672239.5 & 557235.2 \\
\hline FLAG & 780028.0 & 3325382.0 & 900547.06237 & 300138.89484 & 3672244.8 & $557595 . c$ \\
\hline FLAG & 780028.0 & 3325382.4 & 900547.06183 & 300138.90960 & 3672244.8 & 557596.5 \\
\hline FLAG & 780028.7 & 3325290.6 & 900547.12421 & 300135.92926 & 3672242.6 & 557295.2 \\
\hline
\end{tabular}




\begin{tabular}{|c|c|c|c|c|c|c|}
\hline \multirow[b]{2}{*}{ Comment } & \multicolumn{2}{|c|}{ UTM (Zone 15) } & \multicolumn{2}{|c|}{ Geographic } & \multicolumn{2}{|c|}{ State Plane, LA South } \\
\hline & $X, m$ & $\mathrm{Y}, \mathrm{m}$ & Longitude & Latitude & X, U.S. $\mathrm{ft}$ & Y, U.S. ft \\
\hline FLAG & 780029.2 & 3325372.9 & 900547.02702 & 300138.59851 & 3672248.2 & 557565.1 \\
\hline FLAG & 780029.9 & 3325344.1 & 900547.02646 & 300137.66356 & 3672249.3 & 557470.7 \\
\hline FLAG & 780030.2 & 3325347.8 & 900547.01462 & 300137.78275 & 3672250.2 & 557482.8 \\
\hline FLAG & 780030.5 & 3325353.6 & 900546.99484 & 300137.97354 & 3672251.7 & 557502. \\
\hline FLAG & 780030.7 & 3325308.0 & 900547.03136 & 300136.49140 & 3672250.1 & 557352.3 \\
\hline FLAG & 780030.8 & 3325360.9 & 900546.97935 & 300138.20781 & 3672252.8 & 557525.7 \\
\hline FLAG & 780031.3 & 3325422.8 & 900546.90246 & 300140.21595 & 3672257.4 & 557728.6 \\
\hline FLAG & 780033.3 & 3325272.9 & 900546.96660 & 300135.35127 & 3672257.1 & 557237.2 \\
\hline FLAG & 780033.6 & 3325325.9 & 900546.90533 & 300137.07046 & 3672260.6 & 557410.9 \\
\hline FLAG & 780036.7 & 3325326.3 & 900546.79112 & 300137.08057 & 3672270.6 & 557412.0 \\
\hline FLAG & 780039.8 & 3325273.5 & 900546.72631 & 300135.36414 & 3672278.2 & 557238.7 \\
\hline FLAG & 780041.2 & 3325327.1 & 900546.62312 & 300137.10189 & 3672285.3 & 557414.3 \\
\hline FLAG & 780045.5 & 3325274.3 & 900546.51226 & 300135.38545 & 3672297.0 & 557241.1 \\
\hline FLAG & 780045.7 & 3325390.4 & 900546.39305 & 300139.15489 & 3672303.3 & 557621.9 \\
\hline FLAG & 780050.1 & 3325328.8 & 900546.29047 & 300137.14971 & 3672314.5 & 557419.5 \\
\hline FLAG & 780099.3 & 3325472.7 & 900544.31922 & 300141.77850 & 3672482.7 & 557888.9 \\
\hline FLAG & 780104.7 & 3325475.7 & 900544.11347 & 300141.87337 & 3672500.7 & 557898.7 \\
\hline FLAG & 780111.2 & 3325478.6 & 900543.86828 & 300141.96223 & 3672522.2 & 557907.9 \\
\hline FLAG & 780117.4 & 3325481.6 & 900543.63523 & 300142.05527 & 3672542.6 & 557917.5 \\
\hline FLAG & 780131.7 & 3325488.2 & 900543.09666 & 300142.25499 & 3672589.7 & 557938.2 \\
\hline FLAG & 780137.0 & 3325492.1 & 900542.89406 & 300142.37740 & 3672607.4 & 557950.8 \\
\hline FLAG & 780167.3 & 3325499.2 & 900541.75676 & 300142.58370 & 3672707.1 & 557972.7 \\
\hline FLAG & 780170.6 & 3325494.9 & 900541.63686 & 300142.44231 & 3672717.8 & 557958.5 \\
\hline FLAG & 780172.8 & 3325489.3 & 900541.56186 & 300142.25832 & 3672724.6 & 557940.0 \\
\hline FLAG & 780175.2 & 3325486.5 & 900541.47585 & 300142.16645 & 3672732.2 & 557930.8 \\
\hline FLAG & 780177.2 & 3325483.7 & 900541.40323 & 300142.07209 & 3672738.7 & 557921.4 \\
\hline FLAG & 780245.0 & 3325491.5 & 900538.86719 & 300142.26876 & 3672961.4 & 557943.6 \\
\hline FLAG & 780249.6 & 3325478.0 & 900538.71033 & 300141.82754 & 3672975.7 & 557899.2 \\
\hline FLAG & 780250.2 & 3325475.7 & 900538.68771 & 300141.75363 & 3672977.8 & 557891.8 \\
\hline FLAG & 780351.3 & 3325538.2 & 900534.85842 & 300143.69672 & 3673312.2 & 558091.7 \\
\hline FLAG & 780353.8 & 3325527.6 & 900534.77571 & 300143.35145 & 3673319.9 & 558056. \\
\hline FLAG & 780354.6 & 3325522.9 & 900534.74983 & 300143.19773 & 3673322.3 & 558041.4 \\
\hline FLAG & 780355.7 & 3325518.8 & 900534.71387 & 300143.06574 & 3673325.6 & 558028.1 \\
\hline FLAG & 780356.2 & 3325516.5 & 900534.69764 & 300142.98895 & 3673327.1 & 558020.3 \\
\hline FLAG & 780357.2 & 3325513.9 & 900534.66393 & 300142.90288 & 3673330.2 & 558011.7 \\
\hline FLAG & 780359.2 & 3325503.7 & 900534.59674 & 300142.57139 & 3673336.4 & 557978.3 \\
\hline FLAG & 780360.1 & 3325500.2 & 900534.56765 & 300142.45858 & 3673339.1 & 557966.9 \\
\hline FLAG & 780372.2 & 3325509.0 & 900534.10832 & 300142.73257 & 3673379.2 & 557995. \\
\hline FLAG & 780376.4 & 3325505.8 & 900533.95526 & 300142.62396 & 3673392.8 & 557984.2 \\
\hline FLAG & 780379.8 & 3325502.9 & 900533.82981 & 300142.52989 & 3673403.9 & 557974.8 \\
\hline
\end{tabular}




\begin{tabular}{|c|c|c|c|c|c|c|}
\hline \multirow[b]{2}{*}{ Comment } & \multicolumn{2}{|c|}{ UTM (Zone 15) } & \multicolumn{2}{|c|}{ Geographic } & \multicolumn{2}{|c|}{ State Plane, LA South } \\
\hline & $X, m$ & $\mathrm{Y}, \mathrm{m}$ & Longitude & Latitude & X, U.S. $\mathrm{ft}$ & Y, U.S. ft \\
\hline FLAG & 780382.7 & 3325500.9 & 900533.72336 & 300142.46211 & 3673413.3 & 557968.1 \\
\hline FLAG & 780434.9 & 3325504.8 & 900531.77180 & 300142.54538 & 3673584.8 & 557978.3 \\
\hline FLAG & 780438.0 & 3325528.1 & 900531.63641 & 300143.29816 & 3673595.8 & 558054.5 \\
\hline FLAG & 780438.1 & 3325531.6 & 900531.63063 & 300143.41050 & 3673596.2 & 558065.8 \\
\hline FLAG & 780438.3 & 3325513.7 & 900531.63716 & 300142.83074 & 3673596.3 & 558007.3 \\
\hline FLAG & 780438.8 & 3325551.2 & 900531.58327 & 300144.04708 & 3673599.7 & 558130.2 \\
\hline FLAG & 780439.0 & 3325535.1 & 900531.59351 & 300143.52533 & 3673599.4 & 558077.5 \\
\hline FLAG & 780439.0 & 3325516.8 & 900531.60776 & 300142.93022 & 3673598.8 & 558017.4 \\
\hline FLAG & 780439.1 & 3325539.4 & 900531.58528 & 300143.66391 & 3673599.9 & 558091.5 \\
\hline FLAG & 780439.3 & 3325555.1 & 900531.56336 & 300144.17421 & 3673601.3 & 558143.0 \\
\hline FLAG & 780439.4 & 3325560.8 & 900531.55176 & 300144.35901 & 3673602.1 & 558161.7 \\
\hline FLAG & 780439.5 & 3325520.1 & 900531.58681 & 300143.03677 & 3673600.5 & 558028.1 \\
\hline FLAG & 780439.5 & 3325554.8 & 900531.55338 & 300144.16207 & 3673602.2 & 558141.8 \\
\hline FLAG & 780450.7 & 3325534.8 & 900531.15466 & 300143.50543 & 3673638.0 & 558075.9 \\
\hline FLAG & 780450.9 & 3325536.9 & 900531.14742 & 300143.57195 & 3673638.5 & 558082.6 \\
\hline FLAG & 780451.1 & 3325531.5 & 900531.14341 & 300143.39765 & 3673639.1 & 558065.0 \\
\hline FLAG & 780452.2 & 3325505.1 & 900531.12671 & 300142.53988 & 3673641.5 & 557978.4 \\
\hline FLAG & 780464.2 & 3325511.0 & 900530.67577 & 300142.72062 & 3673680.9 & 557997.1 \\
\hline FLAG & 780464.3 & 3325508.4 & 900530.67544 & 300142.63635 & 3673681.0 & 557988.6 \\
\hline FLAG & 780464.5 & 3325515.5 & 900530.65793 & 300142.86639 & 3673682.3 & 558011.8 \\
\hline FLAG & 780464.7 & 3325522.7 & 900530.64397 & 300143.10046 & 3673683.3 & 558035.5 \\
\hline FLAG & 780465.3 & 3325519.2 & 900530.62591 & 300142.98549 & 3673685.0 & 558023.9 \\
\hline FLAG & 780491.2 & 3325508.3 & 900529.66985 & 300142.61135 & 3673769.5 & 557987.0 \\
\hline FLAG & 780491.3 & 3325509.7 & 900529.66419 & 300142.65827 & 3673769.9 & 557991.7 \\
\hline FLAG & 780492.4 & 3325516.5 & 900529.61995 & 300142.87739 & 3673773.5 & 558013.9 \\
\hline FLAG & 780492.5 & 3325513.7 & 900529.61769 & 300142.78493 & 3673773.8 & 558004.6 \\
\hline FLAG & 780494.1 & 3325530.3 & 900529.54369 & 300143.32174 & 3673779.8 & 558058.9 \\
\hline FLAG & 780494.1 & 3325527.1 & 900529.54645 & 300143.22079 & 3673779.6 & 558048.7 \\
\hline FLAG & 780495.3 & 3325534.1 & 900529.49540 & 300143.44573 & 3673783.9 & 558071.4 \\
\hline FLAG & 780495.7 & 3325538.2 & 900529.47413 & 300143.57744 & 3673785.6 & 558084.8 \\
\hline FLAG & 780506.7 & 3325545.5 & 900529.05744 & 300143.80543 & 3673822.0 & 558108.2 \\
\hline FLAG & 780506.8 & 3325542.0 & 900529.05565 & 300143.69285 & 3673822.3 & 558096.8 \\
\hline FLAG & 780506.9 & 3325539.1 & 900529.05587 & 300143.59721 & 3673822.3 & 558087.2 \\
\hline FLAG & 780506.9 & 3325551.8 & 900529.04269 & 300144.00990 & 3673823.0 & 558128.9 \\
\hline FLAG & 780507.1 & 3325535.5 & 900529.05162 & 300143.48108 & 3673822.8 & 558075.4 \\
\hline FLAG & 780507.4 & 3325514.3 & 900529.06030 & 300142.79192 & 3673822.8 & 558005.8 \\
\hline FLAG & 780507.7 & 3325506.8 & 900529.05682 & 300142.54827 & 3673823.4 & 557981.2 \\
\hline FLAG & 780510.6 & 3325509.3 & 900528.94709 & 300142.62977 & 3673833.0 & 557989.5 \\
\hline FLAG & 780514.5 & 3325511.5 & 900528.79902 & 300142.69536 & 3673845.9 & 557996.3 \\
\hline FLAG & 780517.6 & 3325514.2 & 900528.68161 & 300142.78202 & 3673856.1 & 558005.2 \\
\hline
\end{tabular}




\begin{tabular}{|c|c|c|c|c|c|c|}
\hline \multirow[b]{2}{*}{ Comment } & \multicolumn{2}{|c|}{ UTM (Zone 15) } & \multicolumn{2}{|c|}{ Geographic } & \multicolumn{2}{|c|}{ State Plane, LA South } \\
\hline & $X, m$ & $\mathrm{Y}, \mathrm{m}$ & Longitude & Latitude & X, U.S. ft & Y, U.S. ft \\
\hline FLAG & 780521.3 & 3325515.7 & 900528.54114 & 300142.82738 & 3673868.4 & 558009.9 \\
\hline FLAG & 780543.2 & 3325502.1 & 900527.73622 & 300142.36691 & 3673939.7 & 557964.1 \\
\hline FLAG & 780543.5 & 3325511.1 & 900527.71649 & 300142.65901 & 3673941.1 & 557993.7 \\
\hline FLAG & 780543.7 & 3325505.1 & 900527.71744 & 300142.46591 & 3673941.2 & 557974.2 \\
\hline FLAG & 780543.9 & 3325514.0 & 900527.70098 & 300142.75337 & 3673942.4 & 558003.2 \\
\hline FLAG & 780544.9 & 3325500.2 & 900527.67625 & 300142.30357 & 3673945.0 & 557957.8 \\
\hline FLAG & 780544.9 & 3325526.4 & 900527.65035 & 300143.15394 & 3673946.4 & 558043.7 \\
\hline FLAG & 780545.0 & 3325529.8 & 900527.64491 & 300143.26476 & 3673946.7 & 558054.9 \\
\hline FLAG & 780545.2 & 3325521.6 & 900527.64592 & 300142.99933 & 3673946.9 & 558028.1 \\
\hline FLAG & 780545.3 & 3325533.3 & 900527.63130 & 300143.37826 & 3673947.8 & 558066.4 \\
\hline FLAG & 780547.6 & 3325499.3 & 900527.57475 & 300142.27464 & 3673954.0 & 557955.0 \\
\hline FLAG & 780550.1 & 3325538.6 & 900527.44609 & 300143.54503 & 3673963.9 & 558083.4 \\
\hline FLAG & 780552.5 & 3325504.7 & 900527.38892 & 300142.44345 & 3673970.1 & 557972.2 \\
\hline FLAG & 780553.0 & 3325506.8 & 900527.36926 & 300142.51356 & 3673971.8 & 557979.3 \\
\hline FLAG & 780553.3 & 3325540.5 & 900527.32559 & 300143.60561 & 3673974.4 & 558089.7 \\
\hline FLAG & 780559.1 & 3325543.6 & 900527.10601 & 300143.70001 & 3673993.6 & 558099.4 \\
\hline FLAG & 780560.1 & 3325488.5 & 900527.12089 & 300141.91154 & 3673994.3 & 557918.7 \\
\hline FLAG & 780561.7 & 3325555.5 & 900526.99592 & 300144.08500 & 3674002.9 & 558138.4 \\
\hline FLAG & 780563.2 & 3325487.3 & 900527.00524 & 300141.87025 & 3674004.5 & 557914.7 \\
\hline FLAG & 780563.4 & 3325547.5 & 900526.94301 & 300143.82312 & 3674007.8 & 558112.0 \\
\hline FLAG & 780567.3 & 3325484.0 & 900526.85776 & 300141.76105 & 3674017.6 & 557903.8 \\
\hline FLAG & 780569.7 & 3325486.9 & 900526.76345 & 300141.85396 & 3674025.7 & 557913.3 \\
\hline FLAG & 780570.6 & 3325484.5 & 900526.73275 & 300141.77559 & 3674028.5 & 557905.4 \\
\hline FLAG & 780571.8 & 3325484.1 & 900526.68836 & 300141.75879 & 3674032.4 & 557903.7 \\
\hline FLAG & 780572.6 & 3325489.0 & 900526.65388 & 300141.91705 & 3674035.3 & 557919.7 \\
\hline FLAG & 780573.8 & 3325483.0 & 900526.61605 & 300141.72407 & 3674038.8 & 557900.3 \\
\hline FLAG & 780574.4 & 3325490.5 & 900526.58364 & 300141.96495 & 3674041.4 & 557924.6 \\
\hline FLAG & 780576.2 & 3325491.9 & 900526.51752 & 300142.00848 & 3674047.2 & 557929.1 \\
\hline FLAG & 780578.0 & 3325481.3 & 900526.45908 & 300141.66531 & 3674052.7 & 557894.5 \\
\hline FLAG & 780582.1 & 3325479.5 & 900526.30801 & 300141.60258 & 3674066.0 & 557888.3 \\
\hline FLAG & 780583.6 & 3325498.8 & 900526.23272 & 300142.22773 & 3674072.0 & 557951.5 \\
\hline FLAG & 780584.8 & 3325478.3 & 900526.21062 & 300141.56186 & 3674074.7 & 557884.3 \\
\hline FLAG & 780585.7 & 3325500.9 & 900526.15539 & 300142.29466 & 3674078.7 & 557958.3 \\
\hline FLAG & 780589.0 & 3325503.7 & 900526.02825 & 300142.38080 & 3674089.8 & 557967.2 \\
\hline FLAG & 780589.7 & 3325477.0 & 900526.02769 & 300141.51540 & 3674090.8 & 557879.8 \\
\hline FLAG & 780593.3 & 3325508.2 & 900525.86305 & 300142.52565 & 3674104.1 & 557982.0 \\
\hline FLAG & 780596.2 & 3325475.2 & 900525.78693 & 300141.44965 & 3674112.0 & 557873.3 \\
\hline FLAG & 780597.7 & 3325511.4 & 900525.69534 & 300142.62519 & 3674118.8 & 557992.2 \\
\hline FLAG & 780602.2 & 3325514.8 & 900525.52418 & 300142.73013 & 3674133.7 & 558002.9 \\
\hline FLAG & 780604.5 & 3325516.8 & 900525.43713 & 300142.79252 & 3674141.3 & 558009.3 \\
\hline
\end{tabular}




\begin{tabular}{|c|c|c|c|c|c|c|}
\hline \multirow[b]{2}{*}{ Comment } & \multicolumn{2}{|c|}{ UTM (Zone 15) } & \multicolumn{2}{|c|}{ Geographic } & \multicolumn{2}{|c|}{ State Plane, LA South } \\
\hline & $X, m$ & $\mathrm{Y}, \mathrm{m}$ & Longitude & Latitude & X, U.S. ft & Y, U.S. ft \\
\hline FLAG & 780638.4 & 3325439.6 & 900524.24576 & 300140.26237 & 3674248.8 & 557754.9 \\
\hline FLAG & 780639.2 & 3325441.4 & 900524.21438 & 300140.31855 & 3674251.5 & 557760.6 \\
\hline FLAG & 780639.9 & 3325444.4 & 900524.18684 & 300140.41570 & 3674253.8 & 557770.4 \\
\hline FLAG & 780641.2 & 3325447.4 & 900524.13633 & 300140.51345 & 3674258.1 & 557780.3 \\
\hline FLAG & 780643.1 & 3325449.4 & 900524.06308 & 300140.57615 & 3674264.5 & 557786.8 \\
\hline FLAG & 780646.9 & 3325458.4 & 900523.91420 & 300140.86340 & 3674277.3 & 557815.9 \\
\hline FLAG & 780648.2 & 3325461.3 & 900523.86140 & 300140.95688 & 3674281.8 & 557825.4 \\
\hline FLAG & 780649.6 & 3325464.1 & 900523.80543 & 300141.04758 & 3674286.6 & 557834.6 \\
\hline FLAG & 780651.6 & 3325469.3 & 900523.72799 & 300141.21522 & 3674293.2 & 557851.6 \\
\hline FLAG & 780652.9 & 3325447.7 & 900523.69964 & 300140.51193 & 3674296.5 & 557780.6 \\
\hline FLAG & 780653.9 & 3325474.8 & 900523.63683 & 300141.39018 & 3674301.1 & 557869.4 \\
\hline FLAG & 780656.7 & 3325443.8 & 900523.56145 & 300140.38378 & 3674308.8 & 557767.8 \\
\hline FLAG & 780657.4 & 3325480.9 & 900523.50094 & 300141.58695 & 3674312.8 & 557889.4 \\
\hline FLAG & 780660.2 & 3325441.4 & 900523.43113 & 300140.30044 & 3674320.3 & 557759.5 \\
\hline FLAG & 780663.1 & 3325439.5 & 900523.32544 & 300140.23735 & 3674329.7 & 557753.2 \\
\hline FLAG & 780664.7 & 3325437.9 & 900523.26748 & 300140.18535 & 3674334.8 & 557748.0 \\
\hline FLAG & 780669.3 & 3325434.8 & 900523.10057 & 300140.07987 & 3674349.6 & 557737.5 \\
\hline FLAG & 780672.1 & 3325432.0 & 900522.99934 & 300139.98682 & 3674358.6 & 557728.2 \\
\hline FLAG & 780674.2 & 3325430.1 & 900522.91995 & 300139.92273 & 3674365.7 & 557721.8 \\
\hline FLAG & 780680.6 & 3325425.7 & 900522.68715 & 300139.77514 & 3674386.3 & 557707.2 \\
\hline FLAG & 780683.8 & 3325423.0 & 900522.56851 & 300139.68384 & 3674396.8 & 557698.0 \\
\hline FLAG & 780685.5 & 3325466.6 & 900522.46544 & 300141.09834 & 3674404.3 & 557841.0 \\
\hline FLAG & 780685.5 & 3325460.4 & 900522.47061 & 300140.89719 & 3674404.1 & 557820.7 \\
\hline FLAG & 780685.9 & 3325461.8 & 900522.45382 & 300140.94315 & 3674405.5 & 557825.4 \\
\hline FLAG & 780690.2 & 3325417.5 & 900522.33538 & 300139.50278 & 3674417.5 & 557680.0 \\
\hline FLAG & 780738.3 & 3325396.0 & 900520.56366 & 300138.76524 & 3674574.1 & 557607.2 \\
\hline FLAG & 780741.6 & 3325400.6 & 900520.43768 & 300138.90985 & 3674585.0 & 557621.9 \\
\hline FLAG & 780743.8 & 3325421.0 & 900520.33494 & 300139.57150 & 3674593.3 & 557688.8 \\
\hline FLAG & 780744.1 & 3325417.8 & 900520.32774 & 300139.46810 & 3674594.0 & 557678.4 \\
\hline FLAG & 780744.5 & 3325405.2 & 900520.32388 & 300139.05912 & 3674594.8 & 557637.1 \\
\hline FLAG & 780746.7 & 3325408.5 & 900520.23888 & 300139.16253 & 3674602.2 & 557647.6 \\
\hline FLAG & 780748.5 & 3325410.6 & 900520.17072 & 300139.22982 & 3674608.1 & 557654.5 \\
\hline FLAG & 780907.1 & 3325387.7 & 900514.27605 & 300138.35523 & 3675127.2 & 557571.8 \\
\hline FLAG & 780907.5 & 3325390.3 & 900514.25905 & 300138.44018 & 3675128.6 & 557580.4 \\
\hline FLAG & 780908.2 & 3325393.5 & 900514.23165 & 300138.54119 & 3675130.9 & 557590.6 \\
\hline FLAG & 780908.9 & 3325396.5 & 900514.20280 & 300138.64101 & 3675133.3 & 557600.7 \\
\hline FLAG & 780916.9 & 3325347.3 & 900513.95051 & 300137.03536 & 3675157.2 & 557438.7 \\
\hline FLAG & 780917.0 & 3325347.1 & 900513.94535 & 300137.02908 & 3675157.7 & 557438.1 \\
\hline FLAG & 780919.3 & 3325350.4 & 900513.85736 & 300137.13534 & 3675165.3 & 557448.9 \\
\hline FLAG & 780919.5 & 3325350.2 & 900513.85229 & 300137.12897 & 3675165.8 & 557448.3 \\
\hline
\end{tabular}




\begin{tabular}{|c|c|c|c|c|c|c|}
\hline \multirow[b]{2}{*}{ Comment } & \multicolumn{2}{|c|}{ UTM (Zone 15) } & \multicolumn{2}{|c|}{ Geographic } & \multicolumn{2}{|c|}{ State Plane, LA South } \\
\hline & $X, m$ & $\mathrm{Y}, \mathrm{m}$ & Longitude & Latitude & X, U.S. ft & Y, U.S. ft \\
\hline FLAG & 780928.0 & 3325359.2 & 900513.52341 & 300137.41376 & 3675194.4 & 557477.4 \\
\hline FLAG & 780931.6 & 3325363.4 & 900513.38688 & 300137.54787 & 3675206.2 & 557491.1 \\
\hline FLAG & 780935.3 & 3325367.8 & 900513.24476 & 300137.68667 & 3675218.5 & 557505.2 \\
\hline FLAG & 780936.1 & 3325358.4 & 900513.22187 & 300137.38142 & 3675220.9 & 557474.4 \\
\hline FLAG & 780942.0 & 3325375.6 & 900512.98890 & 300137.93454 & 3675240.8 & 557530.5 \\
\hline FLAG & 780942.3 & 3325354.1 & 900512.99573 & 300137.23706 & 3675240.9 & 557460.0 \\
\hline FLAG & 780944.5 & 3325380.3 & 900512.88992 & 300138.08338 & 3675249.3 & 557545.6 \\
\hline FLAG & 780947.9 & 3325349.2 & 900512.79074 & 300137.07262 & 3675259.1 & 557443.6 \\
\hline FLAG & 780948.8 & 3325319.9 & 900512.78620 & 300136.11967 & 3675260.6 & 557347.4 \\
\hline FLAG & 780953.7 & 3325344.9 & 900512.58205 & 300136.92731 & 3675277.6 & 557429.1 \\
\hline FLAG & 780959.2 & 3325340.7 & 900512.38000 & 300136.78735 & 3675295.5 & 557415.2 \\
\hline FLAG & 780961.2 & 3325339.6 & 900512.30548 & 300136.75070 & 3675302.1 & 557411.6 \\
\hline FLAG & 781264.5 & 3325325.4 & 900501.00818 & 300136.03932 & 3676295.9 & 557350.5 \\
\hline FLAG & 781265.3 & 3325327.8 & 900500.97839 & 300136.11642 & 3676298.4 & 557358.3 \\
\hline FLAG & 781265.9 & 3325330.5 & 900500.95408 & 300136.20364 & 3676300.5 & 557367.2 \\
\hline FLAG & 781266.1 & 3325331.9 & 900500.94465 & 300136.24895 & 3676301.3 & 557371.8 \\
\hline FLAG & 781270.6 & 3325345.2 & 900500.76362 & 300136.67684 & 3676316.7 & 557415.2 \\
\hline FLAG & 781270.9 & 3325344.7 & 900500.75431 & 300136.65972 & 3676317.5 & 557413.4 \\
\hline FLAG & 781271.3 & 3325346.9 & 900500.73431 & 300136.73093 & 3676319.2 & 557420.6 \\
\hline FLAG & 781271.6 & 3325347.6 & 900500.72444 & 300136.75464 & 3676320.1 & 557423.1 \\
\hline FLAG & 781273.1 & 3325351.5 & 900500.66349 & 300136.87748 & 3676325.3 & 557435.5 \\
\hline FLAG & 781273.5 & 3325352.3 & 900500.65000 & 300136.90257 & 3676326.4 & 557438.1 \\
\hline FLAG & 781274.4 & 3325354.5 & 900500.61388 & 300136.97495 & 3676329.5 & 557445.4 \\
\hline FLAG & 781275.8 & 3325144.3 & 900500.76040 & 300130.15396 & 3676324.2 & 556756.3 \\
\hline FLAG & 781278.0 & 3325343.5 & 900500.49010 & 300136.61513 & 3676340.8 & 557409.2 \\
\hline FLAG & 781279.2 & 3325345.3 & 900500.44233 & 300136.67163 & 3676345.0 & 557414.9 \\
\hline FLAG & 781279.5 & 3325345.2 & 900500.43345 & 300136.66900 & 3676345.7 & 557414.7 \\
\hline FLAG & 781281.1 & 3325348.4 & 900500.36767 & 300136.76962 & 3676351.4 & 557424.9 \\
\hline FLAG & 781283.1 & 3325351.2 & 900500.29297 & 300136.85887 & 3676357.9 & 557434.0 \\
\hline FLAG & 781283.9 & 3325174.5 & 900500.42999 & 300131.12529 & 3676352.2 & 556854.7 \\
\hline FLAG & 781284.9 & 3325181.7 & 900500.38466 & 300131.36061 & 3676355.9 & 556878.5 \\
\hline FLAG & 781285.0 & 3325158.5 & 900500.40311 & 300130.60720 & 3676355.1 & 556802.4 \\
\hline FLAG & 781285.6 & 3325148.0 & 900500.39281 & 300130.26470 & 3676356.4 & 556767.8 \\
\hline FLAG & 781286.3 & 3325131.7 & 900500.38027 & 300129.73511 & 3676358.1 & 556714.3 \\
\hline FLAG & 781286.8 & 3325357.5 & 900500.14924 & 300137.06289 & 3676370.3 & 557454.7 \\
\hline FLAG & 781287.3 & 3325124.4 & 900500.35014 & 300129.49778 & 3676361.0 & 556690.4 \\
\hline FLAG & 781287.5 & 3325108.3 & 900500.35708 & 300128.97674 & 3676360.9 & 556637.7 \\
\hline FLAG & 781287.6 & 3325117.7 & 900500.34714 & 300129.27902 & 3676361.5 & 556668.3 \\
\hline FLAG & 781287.9 & 3325099.8 & 900500.35154 & 300128.69847 & 3676361.7 & 556609.6 \\
\hline FLAG & 781288.6 & 3325094.1 & 900500.32926 & 300128.51272 & 3676363.9 & 556590.9 \\
\hline
\end{tabular}




\begin{tabular}{|c|c|c|c|c|c|c|}
\hline \multirow[b]{2}{*}{ Comment } & \multicolumn{2}{|c|}{ UTM (Zone 15) } & \multicolumn{2}{|c|}{ Geographic } & \multicolumn{2}{|c|}{ State Plane, LA South } \\
\hline & $\mathrm{X}, \mathrm{m}$ & $\mathrm{Y}, \mathrm{m}$ & Longitude & Latitude & X, U.S. ft & Y, U.S. ft \\
\hline FLAG & 781288.7 & 3325080.0 & 900500.34046 & 300128.05657 & 3676363.4 & 556544.8 \\
\hline FLAG & 781288.7 & 3325089.9 & 900500.33001 & 300128.37900 & 3676364.0 & 556577.4 \\
\hline FLAG & 781289.1 & 3325054.0 & 900500.34930 & 300127.21349 & 3676363.6 & 556459.6 \\
\hline FLAG & 781289.1 & 3325057.9 & 900500.34551 & 300127.33922 & 3676363.7 & 556472.4 \\
\hline FLAG & 781289.1 & 3325067.2 & 900500.33619 & 300127.64203 & 3676364.2 & 556502.9 \\
\hline FLAG & 781291.9 & 3325086.6 & 900500.21562 & 300128.26906 & 3676374.1 & 556566.4 \\
\hline FLAG & 781293.4 & 3325087.0 & 900500.15931 & 300128.27836 & 3676379.1 & 556567.4 \\
\hline FLAG & 781298.8 & 3325078.6 & 900459.96453 & 300128.00382 & 3676396.5 & 556539.8 \\
\hline FLAG & 781299.4 & 3325071.9 & 900459.95112 & 300127.78420 & 3676397.9 & 556517.7 \\
\hline FLAG & 781299.8 & 3325059.7 & 900459.94442 & 300127.38880 & 3676398.9 & 556477.7 \\
\hline FLAG & 781300.1 & 3325065.6 & 900459.92813 & 300127.57937 & 3676400.2 & 556497.0 \\
\hline FLAG & 781321.6 & 3325199.4 & 900459.00142 & 300131.90187 & 3676476.9 & 556934.5 \\
\hline FLAG & 781323.7 & 3325198.8 & 900458.92126 & 300131.88090 & 3676483.9 & 556932.5 \\
\hline FLAG & 781329.4 & 3325197.3 & 900458.71141 & 300131.82790 & 3676502.4 & 556927.3 \\
\hline FLAG & 781409.1 & 3325353.9 & 900455.59173 & 300136.84507 & 3676771.1 & 557437.1 \\
\hline FLAG & 781415.4 & 3325356.9 & 900455.35349 & 300136.93692 & 3676792.0 & 557446.6 \\
\hline FLAG & 781422.2 & 3325374.8 & 900455.08337 & 300137.51164 & 3676815.1 & 557504.9 \\
\hline FLAG & 781422.8 & 3325269.0 & 900455.16048 & 300134.07928 & 3676812.1 & 557158.1 \\
\hline FLAG & 781424.3 & 3325268.1 & 900455.10735 & 300134.04869 & 3676816.8 & 557155.1 \\
\hline FLAG & 781427.5 & 3325385.8 & 900454.87491 & 300137.86282 & 3676833.0 & 557540.6 \\
\hline FLAG & 781432.0 & 3325391.7 & 900454.70204 & 300138.05008 & 3676848.0 & 557559.7 \\
\hline FLAG & 781439.2 & 3325400.9 & 900454.42397 & 300138.34375 & 3676872.1 & 557589.6 \\
\hline FLAG & 781444.4 & 3325319.6 & 900454.30682 & 300135.70126 & 3676885.3 & 557322.8 \\
\hline FLAG & 781446.2 & 3325409.4 & 900454.15312 & 300138.61247 & 3676895.6 & 557617.0 \\
\hline FLAG & 781452.0 & 3325328.9 & 900454.01576 & 300135.99626 & 3676910.6 & 557352.9 \\
\hline FLAG & 781454.7 & 3325415.6 & 900453.83181 & 300138.80764 & 3676923.6 & 557637.0 \\
\hline FLAG & 781457.7 & 3325418.2 & 900453.71741 & 300138.89053 & 3676933.6 & 557645.5 \\
\hline FLAG & 781457.9 & 3325339.3 & 900453.78520 & 300136.32991 & 3676930.5 & 557386.8 \\
\hline FLAG & 781459.0 & 3325348.8 & 900453.73580 & 300136.63673 & 3676934.5 & 557417.8 \\
\hline FLAG & 781460.8 & 3325420.3 & 900453.60036 & 300138.95679 & 3676943.8 & 557652.3 \\
\hline FLAG & 781461.2 & 3325341.0 & 900453.66239 & 300136.38276 & 3676941.2 & 557392.3 \\
\hline FLAG & 781462.9 & 3325350.9 & 900453.58892 & 300136.70110 & 3676947.3 & 557424.5 \\
\hline FLAG & 781464.0 & 3325421.8 & 900453.48071 & 300139.00089 & 3676954.3 & 557656.9 \\
\hline FLAG & 781471.5 & 3325354.3 & 900453.26335 & 300136.80634 & 3676975.8 & 557435.4 \\
\hline FLAG & 781472.0 & 3325419.7 & 900453.18237 & 300138.92689 & 3676980.6 & 557649.7 \\
\hline FLAG & 781476.9 & 3325419.5 & 900453.00052 & 300138.91766 & 3676996.6 & 557649.0 \\
\hline FLAG & 781477.1 & 3325366.0 & 900453.04273 & 300137.18040 & 3676994.8 & 557473.4 \\
\hline FLAG & 781479.6 & 3325375.8 & 900452.94169 & 300137.49474 & 3677003.3 & 557505.3 \\
\hline FLAG & 781481.1 & 3325419.6 & 900452.84535 & 300138.91496 & 3677010.2 & 557648.8 \\
\hline FLAG & 781481.3 & 3325380.7 & 900452.87448 & 300137.65403 & 3677009.1 & 557521.4 \\
\hline
\end{tabular}




\begin{tabular}{|c|c|c|c|c|c|c|}
\hline \multirow[b]{2}{*}{ Comment } & \multicolumn{2}{|c|}{ UTM (Zone 15) } & \multicolumn{2}{|c|}{ Geographic } & \multicolumn{2}{|c|}{ State Plane, LA South } \\
\hline & $X, m$ & $\mathrm{Y}, \mathrm{m}$ & Longitude & Latitude & X, U.S. ft & Y, U.S. ft \\
\hline FLAG & 781484.8 & 3325394.2 & 900452.72994 & 300138.08783 & 3677021.3 & 557565.4 \\
\hline FLAG & 781485.4 & 3325388.6 & 900452.71152 & 300137.90668 & 3677023.1 & 557547.1 \\
\hline FLAG & 781488.3 & 3325394.3 & 900452.59963 & 300138.08965 & 3677032.7 & 557565.7 \\
\hline FLAG & 781489.0 & 3325396.0 & 900452.57305 & 300138.14243 & 3677035.0 & 557571.1 \\
\hline FLAG & 781491.8 & 3325400.4 & 900452.46260 & 300138.28391 & 3677044.6 & 557585.5 \\
\hline FLAG & 781512.7 & 3325423.6 & 900451.66044 & 300139.02021 & 3677114.3 & 557660.6 \\
\hline FLAG & 781540.1 & 3325425.6 & 900450.63641 & 300139.06261 & 3677204.2 & 557665.9 \\
\hline FLAG & 781542.2 & 3325427.6 & 900450.55728 & 300139.12578 & 3677211.1 & 557672.3 \\
\hline FLAG & 781543.5 & 3325354.9 & 900450.57893 & 300136.76528 & 3677211.8 & 557433.9 \\
\hline FLAG & 781545.7 & 3325431.8 & 900450.42446 & 300139.25777 & 3677222.6 & 557685.8 \\
\hline FLAG & 781547.3 & 3325361.1 & 900450.43086 & 300136.96325 & 3677224.6 & 557454.0 \\
\hline FLAG & 781550.6 & 3325367.2 & 900450.30299 & 300137.15684 & 3677235.6 & 557473.7 \\
\hline FLAG & 781551.1 & 3325437.0 & 900450.21841 & 300139.42248 & 3677240.6 & 557702.6 \\
\hline FLAG & 781552.9 & 3325441.9 & 900450.14360 & 300139.57874 & 3677247.0 & 557718.5 \\
\hline FLAG & 781553.9 & 3325375.4 & 900450.16979 & 300137.42248 & 3677247.0 & 557500.6 \\
\hline FLAG & 781555.8 & 3325459.0 & 900450.02088 & 300140.13347 & 3677257.1 & 557774.6 \\
\hline FLAG & 781558.9 & 3325440.0 & 900449.92258 & 300139.51328 & 3677266.5 & 557712.1 \\
\hline FLAG & 781559.4 & 3325384.3 & 900449.95761 & 300137.70443 & 3677265.4 & 557529.3 \\
\hline FLAG & 781561.1 & 3325433.7 & 900449.84819 & 300139.30825 & 3677273.2 & 557691.4 \\
\hline FLAG & 781561.4 & 3325414.9 & 900449.85365 & 300138.69727 & 3677273.4 & 557629.7 \\
\hline FLAG & 781561.9 & 3325421.0 & 900449.83149 & 300138.89295 & 3677275.2 & 557649.5 \\
\hline FLAG & 781563.6 & 3325425.0 & 900449.76199 & 300139.02361 & 3677281.1 & 557662.8 \\
\hline FLAG & 781563.8 & 3325380.9 & 900449.79687 & 300137.59138 & 3677279.6 & 557518.1 \\
\hline FLAG & 781564.1 & 3325407.3 & 900449.75915 & 300138.44758 & 3677282.0 & 557604.6 \\
\hline FLAG & 781564.9 & 3325341.2 & 900449.79447 & 300136.30426 & 3677281.3 & 557388. \\
\hline FLAG & 781565.7 & 3325394.6 & 900449.71342 & 300138.03351 & 3677286.5 & $557562 . \varepsilon$ \\
\hline FLAG & 781566.4 & 3325369.0 & 900449.71194 & 300137.20371 & 3677287.5 & 557479.0 \\
\hline FLAG & 781566.4 & 3325365.0 & 900449.71532 & 300137.07327 & 3677287.4 & 557465.8 \\
\hline FLAG & 781567.1 & 3325373.3 & 900449.68074 & 300137.34140 & 3677290.1 & 557492.9 \\
\hline FLAG & 781567.3 & 3325361.0 & 900449.68391 & 300136.94193 & 3677290.3 & 557452.6 \\
\hline FLAG & 781571.6 & 3325340.9 & 900449.54314 & 300136.28643 & 3677303.4 & 557386.5 \\
\hline FLAG & 781572.8 & 3325346.4 & 900449.49527 & 300136.46589 & 3677307.4 & 557404.7 \\
\hline FLAG & 781573.0 & 3325344.5 & 900449.48869 & 300136.40386 & 3677308.0 & 557398.4 \\
\hline FLAG & 781573.5 & 3325355.3 & 900449.45959 & 300136.75237 & 3677310.2 & 557433.6 \\
\hline FLAG & 781574.3 & 3325349.8 & 900449.43376 & 300136.57322 & 3677312.7 & 557415.6 \\
\hline FLAG & 781575.0 & 3325408.5 & 900449.35479 & 300138.47723 & 3677317.5 & 557608.0 \\
\hline FLAG & 781583.3 & 3325425.6 & 900449.02852 & 300139.02476 & 3677345.6 & 557663.6 \\
\hline FLAG & 781592.7 & 3325444.0 & 900448.66107 & 300139.61620 & 3677377.2 & 557723.7 \\
\hline FLAG & 781595.8 & 3325451.0 & 900448.53624 & 300139.83953 & 3677388.0 & 557746.4 \\
\hline FLAG & 781602.5 & 3325462.3 & 900448.27649 & 300140.20155 & 3677410.4 & 557783.2 \\
\hline
\end{tabular}




\begin{tabular}{|c|c|c|c|c|c|c|}
\hline \multirow[b]{2}{*}{ Comment } & \multicolumn{2}{|c|}{ UTM (Zone 15) } & \multicolumn{2}{|c|}{ Geographic } & \multicolumn{2}{|c|}{ State Plane, LA South } \\
\hline & $X, m$ & $\mathrm{Y}, \mathrm{m}$ & Longitude & Latitude & X, U.S. ft & Y, U.S. ft \\
\hline FLAG & 781607.0 & 3325470.8 & 900448.10123 & 300140.47152 & 3677425.5 & 557810.6 \\
\hline FLAG & 781612.3 & 3325480.4 & 900447.89335 & 300140.77972 & 3677443.4 & 557842.0 \\
\hline FLAG & 781615.1 & 3325487.9 & 900447.78348 & 300141.01959 & 3677452.8 & 557866.3 \\
\hline FLAG & 781616.8 & 3325404.6 & 900447.79804 & 300138.31522 & 3677454.5 & 557593.1 \\
\hline FLAG & 781622.0 & 3325499.8 & 900447.51350 & 300141.40219 & 3677476.1 & 557905.2 \\
\hline FLAG & 781625.5 & 3325507.2 & 900447.37471 & 300141.63816 & 3677488.1 & 557929.2 \\
\hline FLAG & 781629.7 & 3325514.7 & 900447.21363 & 300141.87880 & 3677502.0 & 557953.6 \\
\hline FLAG & 781641.6 & 3325535.0 & 900446.74773 & 300142.52822 & 3677542.2 & 558019.7 \\
\hline FLAG & 781648.4 & 3325548.3 & 900446.48370 & 300142.95450 & 3677564.9 & 558063.0 \\
\hline FLAG & 781657.2 & 3325569.8 & 900446.13546 & 300143.64373 & 3677594.8 & 558132.9 \\
\hline FLAG & 781658.6 & 3325426.4 & 900446.22041 & 300138.99102 & 3677592.4 & 557662.9 \\
\hline FLAG & 781663.2 & 3325432.7 & 900446.04093 & 300139.18966 & 3677608.0 & 557683.1 \\
\hline FLAG & 781665.7 & 3325581.4 & 900445.80774 & 300144.01392 & 3677623.2 & 558170.6 \\
\hline FLAG & 781708.4 & 3325641.2 & 900444.15690 & 300145.91839 & 3677766.2 & 558364.6 \\
\hline FLAG & 781714.8 & 3325654.2 & 900443.90647 & 300146.33259 & 3677787.7 & 558406.7 \\
\hline FLAG & 781715.6 & 3325637.0 & 900443.89253 & 300145.77378 & 3677789.6 & 558350.3 \\
\hline FLAG & 781718.5 & 3325635.1 & 900443.78654 & 300145.71114 & 3677798.9 & 558344.0 \\
\hline FLAG & 781722.6 & 3325649.1 & 900443.61961 & 300146.16077 & 3677813.1 & 558389.6 \\
\hline FLAG & 781724.0 & 3325631.9 & 900443.58468 & 300145.60422 & 3677816.8 & 558333.4 \\
\hline FLAG & 781725.1 & 3325647.2 & 900443.52988 & 300146.09955 & 3677821.1 & 558383.5 \\
\hline FLAG & 781728.6 & 3325628.7 & 900443.41783 & 300145.49428 & 3677831.6 & 558322.5 \\
\hline FLAG & 781729.9 & 3325643.5 & 900443.35469 & 300145.97388 & 3677836.6 & 558371.0 \\
\hline FLAG & 781730.7 & 3325627.0 & 900443.34037 & 300145.43730 & 3677838.5 & 558316.8 \\
\hline FLAG & 781733.0 & 3325626.0 & 900443.25278 & 300145.40477 & 3677846.2 & 558313.6 \\
\hline FLAG & 781734.6 & 3325640.1 & 900443.17991 & 300145.85980 & 3677852.1 & 558359.6 \\
\hline FLAG & 781736.7 & 3325623.8 & 900443.11682 & 300145.32957 & 3677858.2 & 558306.1 \\
\hline FLAG & 781736.8 & 3325638.6 & 900443.09935 & 300145.80895 & 3677859.2 & 558354.6 \\
\hline FLAG & 781740.6 & 3325637.2 & 900442.96177 & 300145.76106 & 3677871.4 & 558349.9 \\
\hline FLAG & 781742.0 & 3325620.1 & 900442.92333 & 300145.20592 & 3677875.4 & 558293.8 \\
\hline FLAG & 781743.3 & 3325635.2 & 900442.86159 & 300145.69347 & 3677880.3 & 558343.1 \\
\hline FLAG & 781748.2 & 3325631.7 & 900442.68394 & 300145.57540 & 3677896.0 & 558331.4 \\
\hline FLAG & 781750.5 & 3325615.0 & 900442.61260 & 300145.03379 & 3677902.9 & 558276.7 \\
\hline FLAG & 781750.5 & 3325615.4 & 900442.61074 & 300145.04514 & 3677903.0 & 558277.9 \\
\hline FLAG & 781752.5 & 3325613.1 & 900442.53803 & 300144.96973 & 3677909.5 & 558270.3 \\
\hline FLAG & 781753.3 & 3325613.6 & 900442.50865 & 300144.98430 & 3677912.1 & 558271.8 \\
\hline FLAG & 781756.0 & 3325612.3 & 900442.41008 & 300144.93939 & 3677920.8 & 558267.4 \\
\hline FLAG & 781756.2 & 3325612.0 & 900442.40154 & 300144.92920 & 3677921.5 & 558266.4 \\
\hline FLAG & 781758.5 & 3325611.0 & 900442.31621 & 300144.89603 & 3677929.1 & 558263.1 \\
\hline FLAG & 781758.6 & 3325609.9 & 900442.31453 & 300144.86145 & 3677929.3 & 558259.6 \\
\hline FLAG & 781763.0 & 3325623.2 & 900442.13944 & 300145.28684 & 3677944.2 & 558302.8 \\
\hline
\end{tabular}




\begin{tabular}{|c|c|c|c|c|c|c|}
\hline \multirow[b]{2}{*}{ Comment } & \multicolumn{2}{|c|}{ UTM (Zone 15) } & \multicolumn{2}{|c|}{ Geographic } & \multicolumn{2}{|c|}{ State Plane, LA South } \\
\hline & $X, m$ & $\mathrm{Y}, \mathrm{m}$ & Longitude & Latitude & X, U.S. ft & Y, U.S. ft \\
\hline FLAG & 781763.1 & 3325622.9 & 900442.13551 & 300145.27977 & 3677944.5 & 558302.0 \\
\hline FLAG & 781764.7 & 3325622.1 & 900442.07672 & 300145.25120 & 3677949.7 & 558299.2 \\
\hline FLAG & 781765.1 & 3325622.2 & 900442.06062 & 300145.25318 & 3677951.2 & 558299.4 \\
\hline FLAG & 781811.5 & 3325838.0 & 900440.12620 & 300152.21868 & 3678113.5 & 559004.9 \\
\hline FLAG & 781824.6 & 3325843.5 & 900439.63153 & 300152.38658 & 3678156.8 & 559022.3 \\
\hline FLAG & 781847.6 & 3325851.9 & 900438.76663 & 300152.63894 & 3678232.5 & 559048.7 \\
\hline FLAG & 781855.1 & 3325854.8 & 900438.48329 & 300152.72595 & 3678257.3 & 559057.7 \\
\hline FLAG & 781867.2 & 3325859.2 & 900438.02771 & 300152.85875 & 3678297.2 & 559071.6 \\
\hline FLAG & 781874.7 & 3325862.2 & 900437.74600 & 300152.95014 & 3678321.9 & 559081.1 \\
\hline FLAG & 781882.9 & 3325864.0 & 900437.43739 & 300153.00335 & 3678348.9 & 559086.7 \\
\hline FLAG & 781889.1 & 3325866.0 & 900437.20394 & 300153.06234 & 3678369.4 & 559092.9 \\
\hline FLAG & 781893.4 & 3325868.2 & 900437.04384 & 300153.13037 & 3678383.4 & 559100.0 \\
\hline FLAG & 781899.0 & 3325870.4 & 900436.83112 & 300153.19762 & 3678402.0 & 559106.9 \\
\hline FLAG & 781900.4 & 3325832.6 & 900436.81564 & 300151.96917 & 3678404.7 & 558982.9 \\
\hline FLAG & 781907.7 & 3325873.8 & 900436.50582 & 300153.30058 & 3678430.5 & 559117.7 \\
\hline FLAG & 781914.6 & 3325873.0 & 900436.24791 & 300153.26631 & 3678453.2 & 559114.4 \\
\hline FLAG & 781921.9 & 3325869.9 & 900435.97808 & 300153.16141 & 3678477.0 & 559104.1 \\
\hline FLAG & 781922.0 & 3325807.3 & 900436.03268 & 300151.12859 & 3678474.5 & 558898.7 \\
\hline FLAG & 781923.6 & 3325807.8 & 900435.97457 & 300151.14567 & 3678479.6 & 558900.5 \\
\hline FLAG & 781928.1 & 3325808.1 & 900435.80565 & 300151.14958 & 3678494.4 & 558901.1 \\
\hline FLAG & 781928.2 & 3325867.2 & 900435.74618 & 300153.06749 & 3678497.5 & 559094.9 \\
\hline FLAG & 781932.2 & 3325808.8 & 900435.65141 & 300151.17069 & 3678507.9 & 558903.3 \\
\hline FLAG & 781935.5 & 3325862.7 & 900435.47728 & 300152.91577 & 3678521.3 & 559079.8 \\
\hline FLAG & 781938.0 & 3325810.7 & 900435.43596 & 300151.22688 & 3678526.8 & 558909.2 \\
\hline FLAG & 781939.5 & 3325860.9 & 900435.32947 & 300152.85524 & 3678534.4 & 559073.8 \\
\hline FLAG & 781940.2 & 3325813.2 & 900435.35084 & 300151.30576 & 3678534.2 & 558917.3 \\
\hline FLAG & 781941.1 & 3325811.6 & 900435.31642 & 300151.25428 & 3678537.3 & 558912.1 \\
\hline FLAG & 781941.9 & 3325806.9 & 900435.29392 & 300151.10193 & 3678539.4 & 558896.7 \\
\hline FLAG & 781941.9 & 3325791.5 & 900435.30827 & 300150.59950 & 3678538.7 & 558846.0 \\
\hline FLAG & 781942.0 & 3325789.2 & 900435.30598 & 300150.52487 & 3678539.0 & 558838.4 \\
\hline FLAG & 781942.3 & 3325802.9 & 900435.28043 & 300150.97037 & 3678540.8 & 558883.5 \\
\hline FLAG & 781942.4 & 3325793.8 & 900435.28536 & 300150.67438 & 3678540.7 & 558853.6 \\
\hline FLAG & 781942.6 & 3325859.7 & 900435.21526 & 300152.81435 & 3678544.4 & 559069.8 \\
\hline FLAG & 781942.8 & 3325786.9 & 900435.27791 & 300150.45162 & 3678541.6 & 558831.1 \\
\hline FLAG & 781944.3 & 3325811.6 & 900435.20001 & 300151.25027 & 3678547.5 & 558911.8 \\
\hline FLAG & 781947.4 & 3325779.9 & 900435.11326 & 300150.21867 & 3678556.3 & 558807.7 \\
\hline FLAG & 781949.9 & 3325782.4 & 900435.01912 & 300150.29745 & 3678564.5 & 558815.7 \\
\hline FLAG & 781951.2 & 3325809.1 & 900434.94220 & 300151.16297 & 3678570.3 & 558903.2 \\
\hline FLAG & 781951.7 & 3325854.0 & 900434.88079 & 300152.62124 & 3678574.1 & 559050.6 \\
\hline FLAG & 781952.5 & 3325784.9 & 900434.91752 & 300150.37734 & 3678573.3 & 558823.9 \\
\hline
\end{tabular}




\begin{tabular}{|c|c|c|c|c|c|c|}
\hline \multirow[b]{2}{*}{ Comment } & \multicolumn{2}{|c|}{ UTM (Zone 15) } & \multicolumn{2}{|c|}{ Geographic } & \multicolumn{2}{|c|}{ State Plane, LA South } \\
\hline & $X, m$ & $\mathrm{Y}, \mathrm{m}$ & Longitude & Latitude & X, U.S. ft & Y, U.S. ft \\
\hline FLAG & 781955.2 & 3325781.0 & 900434.82269 & 300150.24964 & 3678581.8 & 558811.1 \\
\hline FLAG & 781956.4 & 3325852.2 & 900434.70741 & 300152.55822 & 3678589.4 & 559044.4 \\
\hline FLAG & 781957.6 & 3325783.9 & 900434.72775 & 300150.34182 & 3678590.0 & 558820.5 \\
\hline FLAG & 781958.9 & 3325806.5 & 900434.65776 & 300151.07181 & 3678595.4 & 558894.3 \\
\hline FLAG & 781959.0 & 3325850.1 & 900434.61393 & 300152.48751 & 3678597.7 & 559037.4 \\
\hline FLAG & 781960.4 & 3325786.7 & 900434.62094 & 300150.43113 & 3678599.3 & 558829.6 \\
\hline FLAG & 781961.8 & 3325876.8 & 900434.48561 & 300153.35241 & 3678608.0 & 559124.8 \\
\hline FLAG & 781962.0 & 3325779.9 & 900434.57057 & 300150.20758 & 3678604.0 & 558807.1 \\
\hline FLAG & 781962.3 & 3325849.2 & 900434.49328 & 300152.45629 & 3678608.3 & 559034.3 \\
\hline FLAG & 781963.8 & 3325789.6 & 900434.49327 & 300150.52095 & 3678610.4 & 558838.8 \\
\hline FLAG & 781964.4 & 3325874.0 & 900434.38977 & 300153.26044 & 3678616.5 & 559115.6 \\
\hline FLAG & 781965.9 & 3325803.8 & 900434.40219 & 300150.98087 & 3678617.9 & 558885.4 \\
\hline FLAG & 781966.7 & 3325791.4 & 900434.38222 & 300150.57816 & 3678620.1 & 558844.7 \\
\hline FLAG & 781967.1 & 3325871.2 & 900434.29017 & 300153.16689 & 3678625.4 & 559106.3 \\
\hline FLAG & 781967.9 & 3325845.1 & 900434.28815 & 300152.31728 & 3678626.5 & 559020.5 \\
\hline FLAG & 781969.2 & 3325801.9 & 900434.27780 & 300150.91541 & 3678628.9 & 558878.9 \\
\hline FLAG & 781969.8 & 3325792.9 & 900434.26721 & 300150.62326 & 3678630.2 & 558849.4 \\
\hline FLAG & 781969.9 & 3325867.5 & 900434.19047 & 300153.04231 & 3678634.3 & 559093.8 \\
\hline FLAG & 781970.1 & 3325845.1 & 900434.20291 & 300152.31665 & 3678634.0 & 559020.5 \\
\hline FLAG & 781971.1 & 3325795.0 & 900434.21574 & 300150.69111 & 3678634.6 & 558856.3 \\
\hline FLAG & 781972.6 & 3325862.4 & 900434.09419 & 300152.87637 & 3678642.9 & 559077.1 \\
\hline FLAG & 781972.7 & 3325801.6 & 900434.14791 & 300150.90310 & 3678640.4 & 558877.8 \\
\hline FLAG & 781973.7 & 3325797.9 & 900434.11565 & 300150.78063 & 3678643.3 & 558865.4 \\
\hline FLAG & 781973.8 & 3325836.7 & 900434.07543 & 300152.04151 & 3678645.5 & 558992.8 \\
\hline FLAG & 781974.2 & 3325809.8 & 900434.08455 & 300151.16718 & 3678645.6 & 558904.5 \\
\hline FLAG & 781974.5 & 3325811.9 & 900434.07256 & 300151.23623 & 3678646.6 & 558911.5 \\
\hline FLAG & 781974.8 & 3325822.0 & 900434.05227 & 300151.56353 & 3678648.0 & 558944.6 \\
\hline FLAG & 781974.8 & 3325819.0 & 900434.05426 & 300151.46553 & 3678648.0 & 558934.7 \\
\hline FLAG & 781974.9 & 3325814.3 & 900434.05420 & 300151.31267 & 3678648.2 & 558919.2 \\
\hline FLAG & 781975.4 & 3325841.8 & 900434.00896 & 300152.20487 & 3678651.1 & 559009.4 \\
\hline FLAG & 781976.4 & 3325858.6 & 900433.95625 & 300152.74781 & 3678655.2 & 559064.3 \\
\hline FLAG & 781976.9 & 3325800.0 & 900433.99444 & 300150.84592 & 3678653.9 & 558872.1 \\
\hline FLAG & 781977.0 & 3325840.5 & 900433.95096 & 300152.16215 & 3678656.3 & 559005.1 \\
\hline FLAG & 781978.8 & 3325856.3 & 900433.87121 & 300152.67313 & 3678662.7 & 559056.8 \\
\hline FLAG & 781978.8 & 3325805.8 & 900433.91832 & 300151.03403 & 3678660.4 & 558891.2 \\
\hline FLAG & 781980.3 & 3325854.2 & 900433.81468 & 300152.60191 & 3678667.8 & 559049.7 \\
\hline FLAG & 781980.5 & 3325802.2 & 900433.85753 & 300150.91739 & 3678665.9 & 558879.5 \\
\hline FLAG & 781980.6 & 3325840.0 & 900433.81672 & 300152.14378 & 3678668.1 & 559003.4 \\
\hline FLAG & 781982.4 & 3325839.4 & 900433.75210 & 300152.12214 & 3678673.8 & 559001.3 \\
\hline FLAG & 781982.4 & 3325844.7 & 900433.74543 & 300152.29310 & 3678674.2 & 559018.6 \\
\hline
\end{tabular}




\begin{tabular}{|c|c|c|c|c|c|c|}
\hline \multirow[b]{2}{*}{ Comment } & \multicolumn{2}{|c|}{ UTM (Zone 15) } & \multicolumn{2}{|c|}{ Geographic } & \multicolumn{2}{|c|}{ State Plane, LA South } \\
\hline & $\mathrm{X}, \mathrm{m}$ & $\mathrm{Y}, \mathrm{m}$ & Longitude & Latitude & X, U.S. ft & Y, U.S. ft \\
\hline FLAG & 781982.9 & 3325853.1 & 900433.72136 & 300152.56649 & 3678676.0 & 559046.2 \\
\hline FLAG & 781985.4 & 3325809.4 & 900433.66982 & 300151.14532 & 3678682.1 & 558902.7 \\
\hline FLAG & 781985.5 & 3325805.5 & 900433.66865 & 300151.02017 & 3678682.4 & 558890.1 \\
\hline FLAG & 781987.3 & 3325849.9 & 900433.55773 & 300152.45863 & 3678690.5 & 559035.5 \\
\hline FLAG & 781991.6 & 3325852.7 & 900433.39500 & 300152.54530 & 3678704.7 & 559044.4 \\
\hline FLAG & 781992.3 & 3325814.4 & 900433.40770 & 300151.30137 & 3678705.0 & 558918.7 \\
\hline FLAG & 781995.0 & 3325811.0 & 900433.30794 & 300151.18815 & 3678713.9 & 558907.4 \\
\hline FLAG & 781995.1 & 3325841.8 & 900433.27709 & 300152.18736 & 3678715.5 & 559008.3 \\
\hline FLAG & 781997.2 & 3325813.0 & 900433.22441 & 300151.25369 & 3678721.2 & 558914.1 \\
\hline FLAG & 781999.3 & 3325815.2 & 900433.14305 & 300151.32263 & 3678728.2 & 558921.1 \\
\hline FLAG & 782000.9 & 3325843.0 & 900433.05853 & 300152.22217 & 3678734.7 & 559012.1 \\
\hline FLAG & 782001.3 & 3325817.4 & 900433.06742 & 300151.39241 & 3678734.8 & 558928.2 \\
\hline FLAG & 782002.9 & 3325820.9 & 900433.00606 & 300151.50497 & 3678740.1 & 558939.7 \\
\hline FLAG & 782015.1 & 3325850.8 & 900432.52212 & 300152.46439 & 3678781.5 & 559037.0 \\
\hline FLAG & 782022.0 & 3325849.6 & 900432.26643 & 300152.42088 & 3678804.1 & 559032.9 \\
\hline FLAG & 782023.9 & 3325850.5 & 900432.19287 & 300152.44674 & 3678810.5 & 559035.6 \\
\hline FLAG & 782028.7 & 3325853.2 & 900432.01089 & 300152.52919 & 3678826.4 & 559044.1 \\
\hline FLAG & 782033.6 & 3325850.3 & 900431.83050 & 300152.43215 & 3678842.4 & 559034.5 \\
\hline FLAG & 782034.9 & 3325849.2 & 900431.78617 & 300152.39526 & 3678846.3 & 559030.8 \\
\hline FLAG & 782036.4 & 3325848.5 & 900431.72853 & 300152.37096 & 3678851.4 & 559028.4 \\
\hline FLAG & 782037.8 & 3325847.6 & 900431.67907 & 300152.33992 & 3678855.8 & 559025.3 \\
\hline FLAG & 782040.3 & 3325833.8 & 900431.59731 & 300151.89197 & 3678863.5 & 558980.1 \\
\hline FLAG & 782040.6 & 3325846.3 & 900431.57597 & 300152.29695 & 3678864.9 & 559021.0 \\
\hline FLAG & 782042.5 & 3325790.9 & 900431.55551 & 300150.49677 & 3678868.7 & 558839.2 \\
\hline FLAG & 782043.2 & 3325845.2 & 900431.48030 & 300152.25992 & 3678873.3 & 559017.4 \\
\hline FLAG & 782044.0 & 3325838.8 & 900431.45601 & 300152.05045 & 3678875.7 & 558996.3 \\
\hline FLAG & 782044.0 & 3325770.8 & 900431.52008 & 300149.84579 & 3678872.5 & 558773.5 \\
\hline FLAG & 782044.1 & 3325843.9 & 900431.44672 & 300152.21660 & 3678876.3 & 559013.0 \\
\hline FLAG & 782045.9 & 3325843.0 & 900431.37964 & 300152.18592 & 3678882.3 & 559010.0 \\
\hline FLAG & 782046.7 & 3325843.1 & 900431.34902 & 300152.18664 & 3678885.0 & 559010.1 \\
\hline FLAG & 782047.2 & 3325772.5 & 900431.39710 & 300149.89780 & 3678883.3 & 558778.9 \\
\hline FLAG & 782050.0 & 3325773.8 & 900431.29499 & 300149.93812 & 3678892.2 & 558783.0 \\
\hline FLAG & 782051.2 & 3325848.0 & 900431.17770 & 300152.34464 & 3678899.8 & 559026.2 \\
\hline FLAG & 782051.6 & 3325840.8 & 900431.16873 & 300152.10915 & 3678900.9 & 559002.5 \\
\hline FLAG & 782052.7 & 3325848.9 & 900431.12160 & 300152.37060 & 3678904.7 & 559028.9 \\
\hline FLAG & 782052.7 & 3325790.7 & 900431.17690 & 300150.48364 & 3678902.0 & 558838.3 \\
\hline FLAG & 782053.9 & 3325888.1 & 900431.04054 & 300153.64372 & 3678910.4 & 559157.6 \\
\hline FLAG & 782055.0 & 3325851.6 & 900431.03324 & 300152.45838 & 3678912.4 & 559037.9 \\
\hline FLAG & 782057.7 & 3325798.3 & 900430.98240 & 300150.72473 & 3678918.8 & 558862.8 \\
\hline FLAG & 782058.1 & 3325886.8 & 900430.88444 & 300153.59609 & 3678924.2 & 559152.9 \\
\hline
\end{tabular}




\begin{tabular}{|c|c|c|c|c|c|c|}
\hline \multirow[b]{2}{*}{ Comment } & \multicolumn{2}{|c|}{ UTM (Zone 15) } & \multicolumn{2}{|c|}{ Geographic } & \multicolumn{2}{|c|}{ State Plane, LA South } \\
\hline & $X, m$ & $\mathrm{Y}, \mathrm{m}$ & Longitude & Latitude & X, U.S. $\mathrm{ft}$ & Y, U.S. ft \\
\hline FLAG & 782063.8 & 3325884.1 & 900430.67177 & 300153.50279 & 3678943.0 & 559143.7 \\
\hline FLAG & 782068.9 & 3325880.5 & 900430.48757 & 300153.38162 & 3678959.3 & 559131.7 \\
\hline FLAG & 782072.9 & 3325866.4 & 900430.35238 & 300152.92202 & 3678971.7 & 559085.4 \\
\hline FLAG & 782073.8 & 3325800.8 & 900430.38032 & 300150.79419 & 3678971.6 & 558870.4 \\
\hline FLAG & 782073.8 & 3325800.7 & 900430.37829 & 300150.79090 & 3678971.8 & 558870.1 \\
\hline FLAG & 782074.6 & 3325855.4 & 900430.30003 & 300152.56268 & 3678976.7 & 559049.1 \\
\hline FLAG & 782075.9 & 3325848.8 & 900430.25543 & 300152.34731 & 3678980.9 & 559027.4 \\
\hline FLAG & 782076.9 & 3325801.3 & 900430.26436 & 300150.80661 & 3678981.8 & 558871.8 \\
\hline FLAG & 782078.4 & 3325872.7 & 900430.14088 & 300153.12053 & 3678990.1 & 559105.6 \\
\hline FLAG & 782079.4 & 3325802.3 & 900430.17040 & 300150.83861 & 3678990.0 & 558875.1 \\
\hline FLAG & 782080.5 & 3325870.9 & 900430.06291 & 300153.06159 & 3678997.0 & 559099.7 \\
\hline FLAG & 782081.0 & 3325810.8 & 900430.10345 & 300151.11215 & 3678995.6 & 558902.8 \\
\hline FLAG & 782082.3 & 3325803.6 & 900430.06180 & 300150.87610 & 3678999.5 & 558879.0 \\
\hline FLAG & 782084.6 & 3325861.5 & 900429.91832 & 300152.75428 & 3679010.1 & 559068.8 \\
\hline FLAG & 782086.1 & 3325857.9 & 900429.86520 & 300152.63592 & 3679014.9 & 559056.9 \\
\hline FLAG & 782087.2 & 3325853.9 & 900429.82876 & 300152.50580 & 3679018.2 & 559043.8 \\
\hline FLAG & 782087.4 & 3325849.6 & 900429.82711 & 300152.36375 & 3679018.5 & 559029.5 \\
\hline FLAG & 782087.5 & 3325847.1 & 900429.82584 & 300152.28401 & 3679018.7 & 559021.4 \\
\hline FLAG & 782095.3 & 3325860.8 & 900429.52234 & 300152.72161 & 3679044.9 & 559065.9 \\
\hline FLAG & 782107.0 & 3325848.4 & 900429.09651 & 300152.30954 & 3679082.8 & 559024.7 \\
\hline FLAG & 782323.8 & | 3325637.4 & 900421.21247 & 300145.28369 & 3679783.6 & 558322.6 \\
\hline FOUNTAIN & 781510.3 & 3325425.9 & 900451.74878 & 300139.09464 & 3677106.4 & 557668.0 \\
\hline GAS & 782048.1 & 3325759.4 & 900431.37610 & 300149.47091 & 3678885.6 & 558735.8 \\
\hline GAS & 782056.3 & 3325762.9 & 900431.06898 & 300149.57638 & 3678912.5 & 558746.7 \\
\hline GAS & 782068.2 & 3325767.7 & 900430.62226 & 300149.72397 & 3678951.6 & 558762.1 \\
\hline GAS & 782073.0 & 3325769.9 & 900430.43891 & 300149.78973 & 3678967.6 & 558768.9 \\
\hline GAS & 782078.3 & 3325772.0 & 900430.23838 & 300149.85491 & 3678985.2 & 558775.7 \\
\hline GAS & 782085.8 & 3325775.1 & 900429.95747 & 300149.94807 & 3679009.7 & 558785.3 \\
\hline GAS & 782085.8 & 3325804.7 & 900429.92835 & 300150.90975 & 3679011.2 & 558882.5 \\
\hline GAS & 782087.5 & 3325799.6 & 900429.86896 & 300150.74421 & 3679016.6 & 558865.8 \\
\hline GAS & 782089.6 & 3325794.0 & 900429.79899 & 300150.56045 & 3679023.0 & 558847.3 \\
\hline GAS & 782091.7 & 3325787.2 & 900429.72533 & 300150.33719 & 3679029.7 & 558824.9 \\
\hline GAS & 782092.7 & 3325783.5 & 900429.69023 & 300150.21495 & 3679032.9 & 558812.6 \\
\hline GAS & 782094.0 & 3325778.2 & 900429.65000 & 300150.04240 & 3679036.7 & 558795.2 \\
\hline GAS & 782095.9 & 3325808.3 & 900429.54809 & 300151.01781 & 3679044.5 & 558893.8 \\
\hline GAS & 782107.6 & 3325812.4 & 900429.10979 & 300151.14293 & 3679082.9 & 558906.8 \\
\hline GAS & 782128.8 & 3325819.5 & 900428.31197 & 300151.35478 & 3679152.8 & 558929.0 \\
\hline GAS & 782137.5 & 3325822.3 & 900427.98362 & 300151.43825 & 3679181.6 & 558937.8 \\
\hline GAS & 782140.9 & 3325811.9 & 900427.86736 & 300151.09671 & 3679192.2 & 558903.4 \\
\hline GAS & 782144.6 & 3325800.9 & 900427.73808 & 300150.73835 & 3679203.9 & 558867.3 \\
\hline
\end{tabular}




\begin{tabular}{|c|c|c|c|c|c|c|}
\hline \multirow[b]{2}{*}{ Comment } & \multicolumn{2}{|c|}{ UTM (Zone 15) } & \multicolumn{2}{|c|}{ Geographic } & \multicolumn{2}{|c|}{ State Plane, LA South } \\
\hline & $\mathrm{X}, \mathrm{m}$ & $\mathrm{Y}, \mathrm{m}$ & Longitude & Latitude & X, U.S. ft & Y, U.S. ft \\
\hline GAS & 782148.1 & 3325789.9 & 900427.61802 & 300150.37870 & 3679214.9 & 558831.1 \\
\hline GAS & 782151.3 & 3325780.3 & 900427.50796 & 300150.06353 & 3679224.9 & 558799.4 \\
\hline GAS VALVE & 782119.5 & 3325816.6 & 900428.65913 & 300151.26706 & 3679122.4 & 558919.8 \\
\hline GRATE & \begin{tabular}{|l|}
779992.2 \\
\end{tabular} & 3325157.3 & 900548.61067 & 300131.63423 & 3672116.6 & 556860.2 \\
\hline GRATE & 780034.8 & 3325424.2 & 900546.77043 & 300140.25881 & 3672269.0 & 557733.1 \\
\hline GRATE & 780231.1 & 3325497.9 & 900539.38049 & 300142.48858 & 3672916.1 & 557965.3 \\
\hline GRATE & 780747.0 & 3325393.1 & 900520.24130 & 300138.66135 & 3674602.5 & 557597.0 \\
\hline GRATE & 780747.4 & 3325393.3 & 900520.22760 & 300138.66796 & 3674603.7 & 557597.7 \\
\hline GRATE & 780965.4 & 3325342.1 & 900512.14581 & 300136.82635 & 3675316.1 & 557419.4 \\
\hline GRATE & 781291.9 & 3325366.1 & 900459.94777 & 300137.33751 & 3676387.7 & 557482.7 \\
\hline GRATE & 781433.0 & 3325320.4 & 900454.72981 & 300135.73711 & 3676848.1 & 557326.0 \\
\hline GRATE & 781518.0 & 3325337.3 & 900451.54667 & 300136.21368 & 3677127.4 & 557377.2 \\
\hline GRATE & 781651.5 & 3325473.6 & 900446.43904 & 300140.52791 & 3677571.5 & 557817.9 \\
\hline GRATE & 781651.6 & 3325464.9 & 900446.44153 & 300140.24598 & 3677571.6 & 557789.4 \\
\hline GRATE & 781655.4 & 3325457.1 & 900446.30894 & 300139.98710 & 3677583.6 & 557763.4 \\
\hline GRATE & 781657.0 & 3325452.2 & 900446.25316 & 300139.82671 & 3677588.6 & 557747.3 \\
\hline GRATE & 781658.6 & 3325447.8 & 900446.19732 & 300139.68261 & 3677593.7 & 557732.8 \\
\hline GRATE & 7816 & 332 & 900446.09865 & 005 & 3677601.2 & 557840.7 \\
\hline GRATE & 781660.6 & 3325442.6 & 900446.13061 & 300139.51334 & 3677599.8 & 557715.7 \\
\hline GRATE & 781668.9 & 3325485.6 & 900445.77786 & 300140.90322 & 3677629.2 & 557856.5 \\
\hline GRATE & 782031.1 & 3325886.0 & 900431.88932 & 300153.59358 & 3678835.9 & 559151.7 \\
\hline GRATE & 782071.9 & 3325874.3 & 900430.38048 & 300153.17836 & 3678969.0 & 559111.2 \\
\hline HUMP & 781974.8 & 3325827.2 & 900434.04598 & 300151.73073 & 3678648.4 & 558961.5 \\
\hline HUMP & 782064.9 & 3325792.1 & 900430.71923 & 300150.51784 & 3678942.2 & 558842.2 \\
\hline HYDRANT & 780133.7 & 3325452.8 & 900543.05394 & 300141.10519 & 3672594.7 & 557822.1 \\
\hline HYDRANT & 781515.6 & 3325420.5 & 900451.55626 & 300138.91732 & 3677123.5 & 557650.3 \\
\hline LAMP & 779710.9 & 3325096.5 & 900559.15497 & 300129.89419 & 3671191.7 & 556674.4 \\
\hline LAMP & 779743.2 & 3325124.3 & 900557.92397 & 300130.76853 & 3671298.9 & 556763.9 \\
\hline LAMP & 779777.7 & 3325153.8 & 900556.61117 & 300131.69807 & 3671413.3 & 556859.0 \\
\hline LAMP & 779813.1 & 3325189.2 & 900555.25810 & 300132.81737 & 3671531.0 & 556973.4 \\
\hline LAMP & 779836.7 & 3325239.7 & 900554.33105 & 300134.43711 & 3671610.7 & 557137.9 \\
\hline LAMP & 780033.0 & 3325436.4 & 900546.82299 & 300140.65566 & 3672263.9 & 557773.1 \\
\hline LAMP & 780039.2 & 3325407.7 & 900546.62064 & 300139.72086 & 3672282.7 & 557678.9 \\
\hline LAMP & 780048.0 & 3325382.4 & 900546.31549 & 300138.89357 & 3672310.4 & 557595.6 \\
\hline LAMP & 780098.2 & 3325479.8 & 900544.35178 & 300142.01172 & 3672479.6 & 557912.5 \\
\hline LAMP & 780146.6 & 3325454.0 & 900542.57144 & 300141.13504 & 3672637.1 & 557825.6 \\
\hline LAMP & 780204.8 & 3325461.8 & 900540.39303 & 300141.33983 & 3672828.3 & 557848.3 \\
\hline LAMP & 780282.2 & 3325471.8 & 900537.49801 & 300141.59899 & 3673082.5 & 557877.3 \\
\hline LAMP & 780389.2 & 3325497.3 & 900533.48449 & 300142.34031 & 3673434.5 & 557956.0 \\
\hline LAMP & 780460.4 & 3325504.9 & 900530.82409 & 300142.52646 & 3673668.1 & 557977.3 \\
\hline
\end{tabular}




\begin{tabular}{|c|c|c|c|c|c|c|}
\hline \multirow[b]{2}{*}{ Comment } & \multicolumn{2}{|c|}{ UTM (Zone 15) } & \multicolumn{2}{|c|}{ Geographic } & \multicolumn{2}{|c|}{ State Plane, LA South } \\
\hline & $X, m$ & $\mathrm{Y}, \mathrm{m}$ & Longitude & Latitude & X, U.S. ft & Y, U.S. ft \\
\hline LAMP & 780574.2 & 3325477.3 & 900526.60367 & 300141.53637 & 3674040.1 & 557881.3 \\
\hline LAMP & 780765.7 & 3325454.9 & 900519.48727 & 300140.65110 & 3674666.6 & 557798.7 \\
\hline LAMP & 780770.5 & 3325417.4 & 900519.34208 & 300139.43075 & 3674680.7 & 557675.6 \\
\hline LAMP & 780794.8 & 3325444.1 & 900518.40950 & 300140.27656 & 3674761.7 & 557761.9 \\
\hline LAMP & 780813.4 & 3325402.7 & 900517.75720 & 300138.91838 & 3674820.6 & 557625.3 \\
\hline LAMP & 780845.0 & 3325378.7 & 900516.59975 & 300138.11447 & 3674923.2 & 557545.2 \\
\hline LAMP & 780873.2 & 3325411.2 & 900515.51854 & 300139.14438 & 3675017.1 & 557650.3 \\
\hline LAMP & 780893.7 & 3325362.4 & 900514.80173 & 300137.54428 & 3675081.9 & 557489.3 \\
\hline LAMP & 780893.8 & 3325362.1 & 900514.79742 & 300137.53727 & 3675082.2 & 557488.6 \\
\hline LAMP & 780928.3 & 3325369.4 & 900513.50530 & 300137.74303 & 3675195.6 & 557510.7 \\
\hline LAMP & 780963.6 & 3325306.8 & 900512.24618 & 300135.68400 & 3675308.5 & 557303.9 \\
\hline LAMP & 780963.8 & 3325306.6 & 900512.23828 & 300135.67853 & 3675309.2 & 557303.3 \\
\hline LAMP & 780982.3 & 3325348.9 & 900511.50996 & 300137.03356 & 3675371.7 & 557440.9 \\
\hline LAMP & 781038.4 & 3325342.1 & 900509.42309 & 300136.76776 & 3675555.5 & 557416.0 \\
\hline LAMP & 781259.2 & 3325323.9 & 900501.20712 & 300135.99360 & 3676278.5 & 557345.7 \\
\hline LAMP & 781277.2 & 3325178.0 & 900500.67671 & 300131.24437 & 3676330.3 & 556866.5 \\
\hline LAMP & 781278.3 & 3325110.1 & 900500.70009 & 300129.04152 & 3676330.7 & 556644.0 \\
\hline LAMP & 781279.4 & 3325075.6 & 900500.69334 & 300127.92158 & 3676332.5 & 556530.8 \\
\hline LAMP & 781293.9 & 3325312.6 & 900459.92520 & 300135.60039 & 3676391.6 & 557307.2 \\
\hline LAMP & 781301.5 & 3325203.9 & 900459.74496 & 300132.06739 & 3676411.3 & 556950.5 \\
\hline LAMP & 781301.8 & 3325093.6 & 900459.83860 & 300128.48683 & 3676407.0 & 556588.8 \\
\hline LAMP & 781404.7 & 3325353.3 & 900455.75393 & 300136.82847 & 3676756.9 & 557435.3 \\
\hline LAMP & 781411.4 & 3325392.4 & 900455.46900 & 300138.09026 & 3676780.5 & 557563.0 \\
\hline LAMP & 781431.5 & 3325201.7 & 900454.89860 & 300131.88836 & 3676837.5 & 556937.1 \\
\hline LAMP & 781432.9 & 3325316.8 & 900454.73773 & 300135.62099 & 3676847.5 & 557314.3 \\
\hline LAMP & 781445.2 & 3325425.2 & 900454.17750 & 300139.12593 & 3676892.9 & 557668.9 \\
\hline LAMP & 781454.3 & 3325356.6 & 900453.90328 & 300136.89298 & 3676919.5 & 557443.6 \\
\hline LAMP & 781474.5 & 3325397.2 & 900453.11258 & 300138.19327 & 3676987.5 & 557575.7 \\
\hline LAMP & 781482.2 & 3325447.1 & 900452.77711 & 300139.80792 & 3677015.2 & 557739.1 \\
\hline LAMP & 781496.4 & 3325437.1 & 900452.25721 & 300139.47203 & 3677061.3 & 557705.7 \\
\hline LAMP & 781985.2 & 3325791.7 & 900433.69430 & 300150.57123 & 3678680.6 & 558844.7 \\
\hline LAMP & 782016.9 & 3325811.9 & 900432.49253 & 300151.19982 & 3678785.5 & 558909.3 \\
\hline LAMP & 782020.6 & 3325832.7 & 900432.33219 & 300151.87163 & 3678798.9 & 558977.3 \\
\hline LAMP & 782061.3 & 3325810.8 & 900430.83675 & 300151.12785 & 3678931.2 & 558903.7 \\
\hline LAMP & 782069.6 & 3325834.4 & 900430.50635 & 300151.88689 & 3678959.3 & 558980.6 \\
\hline LAMP & 782082.3 & 3325867.9 & 900429.99731 & 300152.96263 & 3679002.9 & 559089.8 \\
\hline LAMP & 782098.3 & 3325823.4 & 900429.44500 & 300151.50519 & 3679053.1 & 558943.1 \\
\hline LAMP POLE & 781652.5 & 3325379.3 & 900446.49034 & 300137.46670 & 3677570.4 & 557508.6 \\
\hline LAMP POLE & 782004.8 & 3325854.6 & 900432.89993 & 300152.59471 & 3678748.2 & 559049.8 \\
\hline METAL VAULT & 780545.6 & 3325535.1 & 900527.61758 & 300143.43649 & 3673948.9 & 558072.3 \\
\hline
\end{tabular}




\begin{tabular}{|c|c|c|c|c|c|c|}
\hline \multirow[b]{2}{*}{ Comment } & \multicolumn{2}{|c|}{ UTM (Zone 15) } & \multicolumn{2}{|c|}{ Geographic } & \multicolumn{2}{|c|}{ State Plane, LA South } \\
\hline & $X, m$ & $\mathrm{Y}, \mathrm{m}$ & Longitude & Latitude & X, U.S. ft & Y, U.S. ft \\
\hline METL GRATE & 781468.7 & 3325419.9 & 900453.30592 & 300138.93425 & 3676969.7 & 557650.3 \\
\hline $\mathrm{MH}$ & 780053.5 & 3325323.2 & 900546.16662 & 300136.96707 & 3672325.6 & 557401.2 \\
\hline $\mathrm{MH}$ & 780377.0 & 3325498.3 & 900533.93711 & 300142.38057 & 3673394.6 & 557959.6 \\
\hline MH DRAIN & 779835.6 & 3325239.4 & 900554.37191 & 300134.42678 & 3671607.2 & 557136.8 \\
\hline MH DRAIN & 779955.3 & 3325107.1 & 900550.03466 & 300130.03490 & 3671993.2 & 556697.3 \\
\hline MH DRAIN & 780766.8 & 3325457.7 & 900519.44251 & 300140.74391 & 3674670.4 & 557808.1 \\
\hline MH DRAIN & 781439.2 & 3325162.4 & 900454.64933 & 300130.60627 & 3676860.8 & 556807.8 \\
\hline MH NOPSI & 779779.8 & 3325154.7 & 900556.53200 & 300131.72613 & 3671420.2 & 556861. \\
\hline MH NOPSI & 779958.9 & 3325130.4 & 900549.87621 & 300130.78822 & 3672006.3 & 556773.5 \\
\hline MH NOPSI & 780008.3 & 3325210.3 & 900547.95994 & 300133.34029 & 3672171.9 & 557033.1 \\
\hline MH NOPSI & 780130.5 & 3325455.1 & 900543.16937 & 300141.18377 & 3672584.5 & 557829.9 \\
\hline MH NOPSI & 780190.5 & 3325463.1 & 900540.92791 & 300141.39460 & 3672781.3 & 557853.2 \\
\hline MH NOPSI & 780278.0 & 3325474.4 & 900537.65374 & 300141.68619 & 3673068.7 & 557885.9 \\
\hline MH NOPSI & 780481.8 & 3325498.1 & 900530.03011 & 300142.28932 & 3673738.1 & 557954.1 \\
\hline MH NOPSI & 780550.9 & 3325491.9 & 900527.45878 & 300142.03090 & 3673964.4 & 557930.5 \\
\hline MH NOPSI & 780629.6 & 3325440.1 & 900524.57599 & 300140.28344 & 3674219.7 & 557756.7 \\
\hline MH NOPSI & 780677.2 & 3325428.1 & 900522.81008 & 300139.85588 & 3674375.4 & 557715.2 \\
\hline MH NOPSI & 781288.1 & 3325178.7 & 900500.26881 & 300131.26003 & 3676366.2 & 556868.5 \\
\hline MH NOPSI & 781290.2 & 3325086.7 & 900500.28026 & 300128.27377 & 3676368.5 & 556566.8 \\
\hline MH SD & 780911.3 & 3325405.6 & 900514.10506 & 300138.93264 & 3675141.6 & 557630.2 \\
\hline MH SEWER & 779687.5 & 3325078.6 & 900600.04522 & 300129.33071 & 3671114.0 & 556616.7 \\
\hline MH SEWER & 780449.8 & 3325543.6 & 900531.17955 & 300143.79133 & 3673635.5 & 558104.7 \\
\hline MH SEWER & 780450.1 & 3325540.3 & 900531.17396 & 300143.68514 & 3673636.1 & 558094. \\
\hline MH SEWER & 780456.4 & 3325502.7 & 900530.97517 & 300142.46006 & 3673654.9 & 557970.5 \\
\hline MH WATER & 780155.0 & 3325451.9 & 900542.26184 & 300141.05918 & 3672664.4 & 557818.2 \\
\hline MH WATER & 780223.3 & 3325460.8 & 900539.70700 & 300141.28999 & 3672888.7 & 557844.0 \\
\hline MH WATER & 780400.3 & 3325495.2 & 900533.07214 & 300142.26148 & 3673470.8 & 557948.4 \\
\hline MH WATER & 780400.4 & 3325494.9 & 900533.06899 & 300142.25193 & 3673471.1 & 557947.4 \\
\hline MH WATER & 780593.8 & 3325460.2 & 900525.88928 & 300140.96601 & 3674103.5 & 557824.4 \\
\hline MH WATER & 780595.5 & 3325458.5 & 900525.82869 & 300140.90951 & 3674108.9 & 557818.7 \\
\hline NOPSI & 781941.8 & 3325813.9 & 900435.28822 & 300151.32911 & 3678539.7 & 558919.7 \\
\hline PARK & 782018.3 & 3325840.6 & 900432.41128 & 300152.13118 & 3678791.6 & 559003.5 \\
\hline PARK & 782023.5 & 3325831.4 & 900432.22809 & 300151.82724 & 3678808.1 & 558973.0 \\
\hline PARK & 782029.1 & 3325832.9 & 900432.01583 & 300151.87235 & 3678826.7 & 558977.7 \\
\hline POST & 782008.0 & 3325856.5 & 900432.77965 & 300152.65528 & 3678758.7 & 559056.1 \\
\hline POST & 782009.0 & 3325857.3 & 900432.74205 & 300152.68016 & 3678762.0 & 559058.6 \\
\hline POST & 782010.2 & 3325858.2 & 900432.69872 & 300152.70854 & 3678765.7 & 559061.5 \\
\hline POST & 782011.5 & 3325858.9 & 900432.64599 & 300152.72903 & 3678770.4 & 559063.7 \\
\hline POST & 782012.1 & 3325859.8 & 900432.62552 & 300152.75906 & 3678772.1 & 559066.7 \\
\hline POST & 782015.9 & 3325863.1 & 900432.47806 & 300152.86295 & 3678785.0 & 559077.3 \\
\hline
\end{tabular}




\begin{tabular}{|c|c|c|c|c|c|c|}
\hline \multirow[b]{2}{*}{ Comment } & \multicolumn{2}{|c|}{ UTM (Zone 15) } & \multicolumn{2}{|c|}{ Geographic } & \multicolumn{2}{|c|}{ State Plane, LA South } \\
\hline & $X, m$ & $\mathrm{Y}, \mathrm{m}$ & Longitude & Latitude & X, U.S. $\mathrm{ft}$ & Y, U.S. ft \\
\hline POST METAL & 780808.2 & 3325421.7 & 900517.93404 & 300139.54012 & 3674804.3 & 557688.0 \\
\hline POST METAL & 780812.7 & 3325431.5 & 900517.75403 & 300139.85581 & 3674819.8 & 557720.0 \\
\hline PVC POST & 781707.5 & 3325633.2 & 900444.19825 & 300145.65743 & 3677762.8 & 558338.2 \\
\hline PVC POST CC & 781749.7 & 3325717.2 & 900442.54520 & 300148.35083 & 3677905.1 & 558611.9 \\
\hline PVC POST CC & 781787.2 & 3325802.5 & 900441.06576 & 300151.08708 & 3678032.1 & 558889.7 \\
\hline PVC POST CC & 781832.7 & 3325841.5 & 900439.32983 & 300152.31494 & 3678183.4 & 559015.4 \\
\hline PVC POST CC & 781894.6 & 3325859.7 & 900437.00730 & 300152.85252 & 3678386.9 & 559071.9 \\
\hline ROAD & 782030.3 & 3325816.7 & 900431.98588 & 300151.34601 & 3678829.9 & 558924.6 \\
\hline ROAD & 782033.8 & 3325800.2 & 900431.87306 & 300150.80841 & 3678840.4 & 558870.4 \\
\hline ROAD & 782035.2 & 3325820.5 & 900431.80126 & 300151.46297 & 3678846.0 & 558936.6 \\
\hline ROAD & 782073.0 & 3325817.3 & 900430.39281 & 300151.33037 & 3678969.9 & 558924.5 \\
\hline ROAD & 782103.0 & 3325827.3 & 900429.26708 & 300151.62850 & 3679068.6 & 558955.7 \\
\hline ROAD & 782115.0 & 3325831.3 & 900428.81452 & 300151.74879 & 3679108.2 & 558968.3 \\
\hline SIDEWALK & 781566.2 & 3325340.2 & 900449.74538 & 300136.26817 & 3677285.6 & 557384.5 \\
\hline SIDEWALK & 781566.9 & 3325345.5 & 900449.71676 & 300136.44006 & 3677288.0 & 557401.8 \\
\hline SIDEWALK & 781572.8 & 3325347.4 & 900449.49222 & 300136.49848 & 3677307.6 & 557408.0 \\
\hline SIDEWALK & 781609.7 & 3325358.9 & 900448.10520 & 300136.83823 & 3677429.2 & 557443.6 \\
\hline SIDEWALK & 781667.6 & 3325402.4 & 900445.90697 & 300138.20176 & 3677620.9 & 557583.5 \\
\hline SIDEWALK & 781709.0 & 3325644.8 & 900444.13184 & 300146.03371 & 3677768.2 & 558376.3 \\
\hline SIDEWALK & 781719.5 & 3325639.5 & 900443.74616 & 300145.85245 & 3677802.3 & 558358.3 \\
\hline SIDEWALK & 781733.3 & 3325630.9 & 900443.23967 & 300145.56421 & 3677847.2 & 558329.7 \\
\hline SIG LIGHT & 782089.5 & 3325842.2 & 900429.75483 & 300152.12406 & 3679025.1 & 559005.3 \\
\hline SIGN & 779723.9 & 3325106.8 & 900558.66136 & 300130.21766 & 3671234.7 & 556707.6 \\
\hline SIGN & 781032.1 & 3325291.4 & 900509.70833 & 300135.12776 & 3675532.2 & 557250.1 \\
\hline SIGN & 781274.0 & 3325322.7 & 900500.65761 & 300135.94463 & 3676326.8 & 557341.3 \\
\hline SIGN & 781287.9 & 3325082.6 & 900500.36890 & 300128.14282 & 3676360.8 & 556553.5 \\
\hline SIGN & 782017.4 & 3325863.4 & 900432.42504 & 300152.87190 & 3678789.6 & 559078.3 \\
\hline SIGN & 782033.1 & 3325823.3 & 900431.87659 & 300151.55705 & 3678839.3 & 558946.0 \\
\hline SIGN & 782039.4 & 3325830.7 & 900431.63469 & 300151.79066 & 3678860.3 & 558969.8 \\
\hline SIGN & 782039.9 & 3325830.6 & 900431.61749 & 300151.78797 & 3678861.8 & 558969.6 \\
\hline SIGN POST & 781585.2 & 3325341.4 & 900449.03684 & 300136.29077 & 3677347.9 & 557387.4 \\
\hline SS & 781993.8 & 3325795.3 & 900433.36924 & 300150.68147 & 3678709.1 & 558856.1 \\
\hline SS & 782012.2 & 3325849.3 & 900432.63248 & 300152.41785 & 3678771.9 & 559032.2 \\
\hline SS & 782088.9 & 3325822.8 & 900429.79635 & 300151.49333 & 3679022.2 & 558941.6 \\
\hline SS DRAIN & 779712.9 & 3325099.3 & 900559.07825 & 300129.98283 & 3671198.3 & 556683.4 \\
\hline SS GRATE & 781954.2 & 3325787.8 & 900434.85095 & 300150.47020 & 3678579.1 & 558833.4 \\
\hline SS GRATE & 782092.7 & 3325812.1 & 900429.66623 & 300151.14567 & 3679034.0 & 558906.6 \\
\hline SS INLET & 779680.7 & 3325071.5 & 900600.30624 & 300129.10540 & 3671091.3 & 556593.6 \\
\hline SS INLET & 779752.5 & 3325133.1 & 900557.57073 & 300131.04694 & 3671329.7 & 556792.4 \\
\hline SS INLET & 779790.4 & 3325165.2 & 900556.12596 & 300132.05600 & 3671455.6 & 95.6 \\
\hline
\end{tabular}




\begin{tabular}{|c|c|c|c|c|c|c|}
\hline \multirow[b]{2}{*}{ Comment } & \multicolumn{2}{|c|}{ UTM (Zone 15) } & \multicolumn{2}{|c|}{ Geographic } & \multicolumn{2}{|c|}{ State Plane, LA South } \\
\hline & $\mathrm{X}, \mathrm{m}$ & $\mathrm{Y}, \mathrm{m}$ & Longitude & Latitude & X, U.S. ft & Y, U.S. ft \\
\hline SS INLET & 779822.9 & 3325206.6 & 900554.87696 & 300133.37315 & 3671563.9 & 557029.9 \\
\hline SS INLET & 779840.2 & 3325252.2 & 900554.18739 & 300134.83822 & 3671622.9 & 557178.5 \\
\hline SS INLET & 779965.9 & 3325106.1 & 900549.64060 & 300129.99438 & 3672027.9 & 556693.5 \\
\hline SS INLET & 780034.7 & 3325426.8 & 900546.76932 & 300140.34418 & 3672269.0 & 557741.7 \\
\hline SS INLET & 780036.0 & 3325206.5 & 900546.93106 & 300133.19506 & 3672262.5 & 557019.4 \\
\hline SS INLET & 780061.6 & 3325322.6 & 900545.86697 & 300136.93912 & 3672352.0 & 557398.6 \\
\hline SS INLET & 780812.1 & 3325403.1 & 900517.80639 & 300138.93327 & 3674816.2 & 557626.8 \\
\hline SS INLET & 780914.1 & 3325344.2 & 900514.05625 & 300136.93699 & 3675148.0 & 557428.7 \\
\hline SS INLET & 780914.2 & 3325344.5 & 900514.05388 & 300136.94681 & 3675148.2 & 557429.7 \\
\hline SS INLET & 780946.4 & 3325317.0 & 900512.87780 & 300136.02910 & 3675252.6 & 557338.1 \\
\hline SS INLET & 780946.5 & 3325316.9 & 900512.87659 & 300136.02716 & 3675252.7 & 557337.9 \\
\hline SS INLET & 781270.1 & 3325322.4 & 900500.80544 & 300135.93722 & 3676313.8 & 557340.4 \\
\hline SS INLET & 781277.9 & 3325101.3 & 900500.72247 & 300128.75514 & 3676329.1 & 556615.0 \\
\hline SS INLET & 781286.0 & 3325190.1 & 900500.33592 & 300131.63242 & 3676359.9 & 556906.0 \\
\hline SS INLET & 782022.5 & 3325857.4 & 900432.23990 & 300152.67217 & 3678806.1 & 559058.3 \\
\hline SS INLETS & 782078.3 & 3325836.0 & 900430.17711 & 300151.93096 & 3678988.2 & 558985.4 \\
\hline SS INLT & 781431.0 & 3325212.4 & 900454.91018 & 300132.23536 & 3676836.1 & 556972.1 \\
\hline SS INLT & 7814 & 332 & 955 & 556 & 4.0 & 42.0 \\
\hline SS INLT & \begin{tabular}{|l|l|}
781437.4 \\
\end{tabular} & 3325162.3 & 900454.71713 & 300130.60226 & 3676854.9 & 556807.4 \\
\hline SS INLT & 781440.5 & 3325316.6 & 900454.45522 & 300135.60641 & 3676872.4 & 557313.1 \\
\hline SS INLT & 781494.1 & 3325418.9 & 900452.35789 & 300138.88223 & 3677053.1 & 557646.0 \\
\hline STAIRS & 780904.8 & 3325351.7 & 900514.39597 & 300137.18961 & 3675117.9 & 557453.9 \\
\hline STAIRS & 780905.1 & 3325351.6 & 900514.38580 & 300137.18530 & 3675118.8 & 557453.5 \\
\hline STAIRS & 780922.2 & 3325371.1 & 900513.73161 & 300137.80385 & 3675175.6 & 557516.6 \\
\hline STAIRS & 781001.9 & 3325294.7 & 900510.82974 & 300135.25989 & 3675433.5 & 557262.4 \\
\hline STAIRS & 781002.6 & 3325294.3 & 900510.80614 & 300135.24524 & 3675435.6 & 557260.9 \\
\hline STAIRS & \begin{tabular}{|l|}
781006.3 \\
\end{tabular} & 3325321.9 & 900510.63970 & 300136.13853 & 3675449.2 & 557351.3 \\
\hline STORM DRAIN & 782011.9 & 3325848.8 & 900432.64107 & 300152.40256 & 3678771.2 & 559030.7 \\
\hline STORM DRAIN & 782022.1 & 3325857.1 & 900432.25233 & 300152.66319 & 3678805.0 & 559057.4 \\
\hline TABLE & 781528.5 & 3325424.7 & 900451.07145 & 300139.04129 & 3677166.0 & 557663.3 \\
\hline TREE & 781955.9 & 3325804.4 & 900434.77346 & 300151.00714 & 3678585.3 & 558887.7 \\
\hline TREE & 781962.7 & 3325786.0 & 900434.53840 & 300150.40475 & 3678606.6 & 558827.0 \\
\hline TREE & 781989.5 & 3325808.1 & 900433.51704 & 300151.09923 & 3678695.6 & 558898.2 \\
\hline TREE & \begin{tabular}{|l|}
781993.1 \\
\end{tabular} & 3325810.0 & 900433.37901 & 300151.15775 & 3678707.7 & 558904.2 \\
\hline TREE & 782064.0 & 3325766.4 & 900430.77941 & 300149.68367 & 3678937.8 & 558757.8 \\
\hline TREES & 781940.2 & 3325797.6 & 900435.36347 & 300150.79955 & 3678533.6 & 558866.1 \\
\hline VALVE NOPSI & 781286.9 & 3325097.4 & 900500.39217 & 300128.62096 & 3676358.2 & 556601.8 \\
\hline VALVE NOPSI & 781292.1 & 3325091.8 & 900500.20179 & 300128.43677 & 3676375.2 & 556583.4 \\
\hline VALVE WATER & 780533.7 & 3325492.6 & 900528.10256 & 300142.06677 & 3673907.8 & 557933.5 \\
\hline VALVE WATER & 780633.2 & 3325434.8 & 900524.44514 & 300140.11107 & 3674231.4 & 557739.4 \\
\hline
\end{tabular}




\begin{tabular}{|l|l|l|l|l|l|l|}
\hline \multirow{2}{*}{ Comment } & \multicolumn{2}{|c|}{ UTM (Zone 15) } & \multicolumn{3}{c|}{ Geographic } & \multicolumn{2}{c|}{ State Plane, LA South } \\
\cline { 2 - 7 } & $\mathrm{X}, \mathrm{m}$ & $\mathrm{Y}, \mathrm{m}$ & Longitude & Latitude & X, U.S. ft & Y, U.S. $\mathrm{ft}$ \\
\hline VAULT & 780485.5 & 3325539.4 & 900529.85460 & 300143.62465 & 3673752.1 & 558089.2 \\
\hline VAULT EX & 780090.3 & 3325445.1 & 900544.68083 & 300140.89051 & 3672451.9 & 557798.9 \\
\hline VAULT EX & 780096.3 & 3325478.5 & 900544.42456 & 300141.97017 & 3672473.3 & 557908.2 \\
\hline VAULT EX & 780100.8 & 3325471.9 & 900544.26227 & 300141.75344 & 3672487.8 & 557886.5 \\
\hline VAULT EX & 780101.6 & 3325447.0 & 900544.25794 & 300140.94507 & 3672489.0 & 557804.8 \\
\hline VAULT EX & 780115.9 & 3325448.4 & 900543.72160 & 300140.97760 & 3672536.2 & 557808.6 \\
\hline VAULT EX & 780177.5 & 3325457.3 & 900541.41679 & 300141.21498 & 3672738.5 & 557834.8 \\
\hline VAULT EX & 780283.7 & 3325470.5 & 900537.44528 & 300141.55587 & 3673087.2 & 557873.0 \\
\hline VAULT EX & 780392.2 & 3325496.5 & 900533.37464 & 300142.30992 & 3673444.1 & 557953.0 \\
\hline VERT PIPE & 781516.9 & 3325423.6 & 900451.50676 & 300139.01745 & 3677127.8 & 557660.5 \\
\hline W VALVE & 781953.9 & 3325786.1 & 900434.86606 & 300150.41697 & 3678577.8 & 558828.0 \\
\hline WALL & 782136.0 & 3325834.7 & 900428.02978 & 300151.84301 & 3679177.1 & 558978.6 \\
\hline WALL & 782148.3 & 3325799.0 & 900427.60336 & 300150.67168 & 3679215.8 & 558860.7 \\
\hline WALL END & 782153.6 & 3325782.4 & 900427.42182 & 300150.13025 & 3679232.4 & 558806.2 \\
\hline WELL & 782109.6 & 3325756.5 & 900429.08732 & 300149.32641 & 3679086.9 & 558723.4 \\
\hline WX & 782126.8 & 3325832.9 & 900428.37267 & 300151.79181 & 3679147.0 & 558973.1 \\
\hline
\end{tabular}

Note: NAD83.

Table A4. LPV104 flagged anomalies and utility locations.

\begin{tabular}{|l|l|l|l|l|l|l|}
\hline \multirow{2}{*}{ Comment } & \multicolumn{2}{|c|}{ UTM (Zone 15) } & \multicolumn{3}{c|}{ Geographic } & \multicolumn{2}{c|}{ State Plane, LA South } \\
\cline { 2 - 7 } & $\mathrm{X}, \mathrm{m}$ & $\mathrm{Y}, \mathrm{m}$ & Longitude & Latitude & X, U.S. $\mathrm{ft}$ & Y, U.S. ft \\
\hline ANOMALY & 783835.0 & 3326081.0 & 900324.43910 & 300158.41854 & 3684758.8 & 559704.6 \\
\hline ANOMALY & 783839.0 & 3326065.0 & 900324.30530 & 300157.89607 & 3684771.2 & 559652.0 \\
\hline ANOMALY & 783860.0 & 3326061.0 & 900323.52607 & 300157.74876 & 3684839.8 & 559637.9 \\
\hline ANOMALY & 783879.0 & 3326063.0 & 900322.81567 & 300157.79778 & 3684902.2 & 559643.5 \\
\hline ANOMALY & 783896.0 & 3326066.0 & 900322.17888 & 300157.88092 & 3684958.1 & 559652.6 \\
\hline ANOMALY & 783916.0 & 3326068.0 & 900321.43118 & 300157.92911 & 3685023.8 & 559658.2 \\
\hline ANOMALY & 783938.0 & 3326071.0 & 900320.60795 & 300158.00807 & 3685096.0 & 559666.9 \\
\hline ANOMALY & 783951.0 & 3326072.0 & 900320.12224 & 300158.02966 & 3685138.7 & 559669.6 \\
\hline ANOMALY & 783970.0 & 3326075.0 & 900319.41087 & 300158.11113 & 3685201.1 & 559678.5 \\
\hline ANOMALY & 783999.0 & 3326078.0 & 900318.32662 & 300158.18424 & 3685296.3 & 559687.0 \\
\hline ANOMALY & 784012.0 & 3326080.0 & 900317.83995 & 300158.23827 & 3685339.0 & 559692.9 \\
\hline ANOMALY ARE & 785584.8 & 3325944.1 & 900219.32396 & 300152.51142 & 3690488.6 & 559172.2 \\
\hline ASPHALT & 782923.9 & 3326010.4 & 900358.48065 & 300156.88734 & 3681768.6 & 559516.8 \\
\hline ASPHALT & 782928.2 & 3326006.8 & 900358.32375 & 300156.76696 & 3681782.5 & 559504.8 \\
\hline ASPHALT & 782931.1 & 3326008.5 & 900358.21398 & 300156.81970 & 3681792.1 & 559510.2 \\
\hline ASPHALT & 782963.9 & 3325970.5 & 900357.02725 & 300155.55947 & 3681897.8 & 559384.1 \\
\hline ASPHALT & 782970.3 & 3325969.9 & 900356.78918 & 300155.53467 & 3681918.8 & 559381.8 \\
\hline ASPHALT & 782974.4 & 3325969.0 & 900356.63716 & 300155.50206 & 3681932.2 & 559378.6 \\
\hline
\end{tabular}




\begin{tabular}{|c|c|c|c|c|c|c|}
\hline \multirow[b]{2}{*}{ Comment } & \multicolumn{2}{|c|}{ UTM (Zone 15) } & \multicolumn{2}{|c|}{ Geographic } & \multicolumn{2}{|c|}{ State Plane, LA South } \\
\hline & $\mathrm{X}, \mathrm{m}$ & $\mathrm{Y}, \mathrm{m}$ & Longitude & Latitude & X, U.S. $\mathrm{ft}$ & Y, U.S. ft \\
\hline ASPHALT EDG & 785359.8 & 3326172.3 & 900227.49354 & 300200.10428 & 3689761.9 & 559931.0 \\
\hline ASPHALT ROAD & 785352.5 & 3326075.0 & 900227.85961 & 300156.95352 & 3689733.3 & 559612.4 \\
\hline BUSH END & 785554.7 & 3325909.0 & 900220.48020 & 300151.39788 & 3690388.3 & 559058.5 \\
\hline BUSH END & 785576.5 & 3325908.4 & 900219.66791 & 300151.36011 & 3690459.7 & 559055.5 \\
\hline CABLE DIG H & 785579.6 & 3325961.8 & 900219.50077 & 300153.09006 & 3690472.4 & 559230.4 \\
\hline CABLE DIG H & 785586.6 & 3325996.4 & 900219.20635 & 300154.20677 & 3690497.0 & 559343.5 \\
\hline CABLE TRASH & 785561.3 & 3325940.3 & 900220.20389 & 300152.40787 & 3690411.4 & 559160.8 \\
\hline CENTER LINE & 783214.5 & 3326054.3 & 900347.60246 & 300158.06974 & 3682723.4 & 559646.8 \\
\hline CONCRETE & 783140.4 & 3326061.1 & 900350.35906 & 300158.35209 & 3682480.8 & 559672.6 \\
\hline CONCRETE & 784987.1 & 3326269.5 & 900241.29733 & 300203.57059 & 3688544.8 & 560267.5 \\
\hline CONCRETE SL & 785029.1 & 3326284.7 & 900239.71655 & 300204.02854 & 3688683.2 & 560315.3 \\
\hline CONCRETE WA & 785139.5 & 3326281.9 & 900235.60255 & 300203.84511 & 3689045.0 & 560300.9 \\
\hline CONDUIT & 785112.8 & 3326273.5 & 900236.60626 & 300203.59496 & 3688957.0 & 560274.6 \\
\hline CURB & 785222.3 & 3326200.6 & 900232.59342 & 300201.13787 & 3689312.5 & 560030.4 \\
\hline CURB & 785225.1 & 3326194.1 & 900232.49528 & 300200.92462 & 3689321.4 & 560008.9 \\
\hline CURB & 785243.0 & 3326194.2 & 900231.82772 & 300200.91285 & 3689380.1 & 560008.4 \\
\hline CURB & 785249.5 & 3326186.9 & 900231.59238 & 300200.67055 & 3689401.0 & 559984.2 \\
\hline CURB & 785264.6 & 3326156.5 & 900231.05864 & 300159.67155 & 3689449.1 & 559883.8 \\
\hline CURB & 785266.2 & 3326181.8 & 900230.97458 & 300200.49107 & 3689455.5 & 559966.7 \\
\hline CURB & 785274.2 & 3326184.2 & 900230.67396 & 300200.56222 & 3689481.9 & 559974.1 \\
\hline CURB & 785274.7 & 3326176.9 & 900230.66236 & 300200.32495 & 3689483.2 & 559950.2 \\
\hline CURB & 785277.2 & 3326158.3 & 900230.58707 & 300159.71938 & 3689490.5 & 559889.1 \\
\hline CURB & 785277.7 & 3326173.0 & 900230.55425 & 300200.19590 & 3689492.8 & 559937.3 \\
\hline CURB & 785279.7 & 3326166.8 & 900230.48565 & 300159.99306 & 3689499.1 & 559916.8 \\
\hline CURB & 785280.2 & 3326162.0 & 900230.47164 & 300159.83691 & 3689500.5 & 559901.1 \\
\hline CURB & 785281.3 & 3326179.9 & 900230.41336 & 300200.41675 & 3689504.9 & 559959.7 \\
\hline CURB & 785284.8 & 3326172.0 & 900230.29047 & 300200.15750 & 3689516.0 & 559933.6 \\
\hline CURB & 785286.7 & 3326166.1 & 900230.22531 & 300159.96448 & 3689522.0 & 559914.2 \\
\hline CURB & 785287.0 & 3326158.7 & 900230.22126 & 300159.72413 & 3689522.6 & 559889.9 \\
\hline CURB & 785292.6 & 3326158.7 & 900230.01244 & 300159.71943 & 3689541.0 & 559889.7 \\
\hline DIG SPOT & 785595.4 & 3325986.0 & 900218.88826 & 300153.86195 & 3690525.4 & 559309.0 \\
\hline DRAIN & 782305.4 & 3325882.0 & 900421.66650 & 300153.23546 & 3679734.8 & 559125.4 \\
\hline DRAIN & 782472.4 & 3325931.3 & 900415.39220 & 300154.69633 & 3680284.6 & 559279.0 \\
\hline DRAIN & 782931.0 & 3325995.1 & 900358.23053 & 300156.38501 & 3681791.1 & 559466.3 \\
\hline DRAIN & 784206.2 & 3326202.1 & 900310.48117 & 300202.03754 & 3685981.5 & 560083.9 \\
\hline DRAIN & 784233.9 & 3326203.7 & 900309.44672 & 300202.06630 & 3686072.4 & 560087.8 \\
\hline DRAIN & 785524.6 & 3325885.6 & 900221.62514 & 300150.66395 & 3690288.5 & 558983.3 \\
\hline DRAIN & 785573.8 & 3325900.3 & 900219.77640 & 300151.09957 & 3690450.5 & 559029.1 \\
\hline DRAIN & 785573.9 & 3325901.3 & 900219.77171 & 300151.13193 & 3690450.8 & 559032.4 \\
\hline DRAIN W/ PI & 785330.6 & 3326205.3 & 900228.55053 & 300201.19948 & 3689667.8 & 560040.6 \\
\hline
\end{tabular}




\begin{tabular}{|c|c|c|c|c|c|c|}
\hline \multirow[b]{2}{*}{ Comment } & \multicolumn{2}{|c|}{ UTM (Zone 15) } & \multicolumn{2}{|c|}{ Geographic } & \multicolumn{2}{|c|}{ State Plane, LA South } \\
\hline & $\mathrm{X}, \mathrm{m}$ & $\mathrm{Y}, \mathrm{m}$ & Longitude & Latitude & X, U.S. ft & Y, U.S. ft \\
\hline DRIVE & 783024.9 & 3325996.7 & 900354.72757 & 300156.35877 & 3682099.0 & 559467.0 \\
\hline DRIVE & 783040.2 & 3325975.9 & 900354.17695 & 300155.67116 & 3682148.2 & 559398.1 \\
\hline DRIVE & 783054.6 & 3325953.6 & 900353.66133 & 300154.93563 & 3682194.4 & 559324.3 \\
\hline DRIVE EDGE & 785338.1 & 3326117.3 & 900228.35576 & 300158.33802 & 3689688.1 & 559751.8 \\
\hline DRIVE EDGE & 785351.6 & 3326110.4 & 900227.85902 & 300158.10282 & 3689732.1 & 559728.5 \\
\hline DRIVE EDGE & 785352.8 & 3326118.5 & 900227.80646 & 300158.36462 & 3689736.4 & 559755.0 \\
\hline DRIVE EDGE & 785373.7 & 3326115.9 & 900227.02964 & 300158.26272 & 3689804.8 & 559745.5 \\
\hline ELEC CABLE & 785599.8 & 3325988.1 & 900218.72217 & 300153.92639 & 3690539.9 & 559315.7 \\
\hline ELEC CABLE & 785604.3 & 3325993.3 & 900218.54935 & 300154.09133 & 3690554.9 & 559332.5 \\
\hline ERDC UTILITY & 785131.5 & 3326277.2 & 900235.90539 & 300203.69933 & 3689018.5 & 560285.8 \\
\hline ERDC WATER & 785187.9 & 3326269.0 & 900233.81020 & 300203.38597 & 3689203.0 & 560256.3 \\
\hline FENCE & 782999.0 & 3325963.8 & 900355.72483 & 300155.31287 & 3682012.6 & 559360.4 \\
\hline FENCE & 783010.2 & 3325935.3 & 900355.33446 & 300154.37884 & 3682047.9 & 559266.4 \\
\hline FENCE & 785323.0 & 3326203.1 & 900228.83605 & 300201.13448 & 3689642.7 & 560033.8 \\
\hline FENCE & 785331.7 & 3326205.8 & 900228.50903 & 300201.21478 & 3689671.4 & 560042.2 \\
\hline FENCE & 785338.6 & 3326193.7 & 900228.26341 & 300200.81640 & 3689693.4 & 560002.2 \\
\hline FENCE & 785356.8 & 3326131.6 & 900227.64467 & 300158.78629 & 3689750.1 & 559797.7 \\
\hline FENCE & 785404.7 & 3326040.8 & 900225.94617 & 300155.80008 & 3689902.8 & 559497.8 \\
\hline FENCE & 785407.3 & 3326036.8 & 900225.85308 & 300155.66812 & 3689911.2 & 559484.6 \\
\hline FENCE & 785474.0 & 3325968.2 & 900223.43217 & 300153.38639 & 3690126.5 & 559256.5 \\
\hline FENCE & 785509.3 & 3325955.8 & 900222.12788 & 300152.95443 & 3690241.7 & 559214.1 \\
\hline FENCE & 785523.4 & 3325965.8 & 900221.59247 & 300153.26704 & 3690288.4 & 559246.2 \\
\hline FENCE & 785540.6 & 3325986.7 & 900220.93095 & 300153.93070 & 3690345.7 & 559313.9 \\
\hline FENCE END W & 785367.8 & 3326110.7 & 900227.25466 & 300158.09896 & 3689785.2 & 559728.7 \\
\hline FENCE LINE & 785319.6 & 3326209.4 & 900228.95675 & 300201.34173 & 3689631.9 & 560054.6 \\
\hline FIRE HYDRAN & 785502.3 & 3325884.5 & 900222.45771 & 300150.64699 & 3690215.3 & 558980.7 \\
\hline FLAG & 782329.8 & 3325876.9 & 900420.76152 & 300153.04972 & 3679814.6 & 559107.5 \\
\hline FLAG & 782332.4 & 3325872.3 & 900420.66896 & 300152.89831 & 3679822.9 & 559092.3 \\
\hline FLAG & 782341.3 & 3325852.6 & 900420.35589 & 300152.25174 & 3679851.1 & 559027.3 \\
\hline FLAG & 782341.4 & 3325878.4 & 900420.32754 & 300153.08876 & 3679852.7 & 559111.9 \\
\hline FLAG & 782342.3 & 3325850.0 & 900420.32108 & 300152.16655 & 3679854.3 & 559018.7 \\
\hline FLAG & 782343.8 & 3325872.9 & 900420.24329 & 300152.90831 & 3679860.3 & 559093.7 \\
\hline FLAG & 782344.0 & 3325847.0 & 900420.26055 & 300152.06780 & 3679859.7 & 559008.8 \\
\hline FLAG & 782345.8 & 3325843.0 & 900420.19725 & 300151.93652 & 3679865.4 & 558995.6 \\
\hline FLAG & 782349.0 & 3325854.3 & 900420.06714 & 300152.30050 & 3679876.4 & 559032.5 \\
\hline FLAG & 782349.9 & 3325851.5 & 900420.03626 & 300152.20891 & 3679879.2 & 559023.3 \\
\hline FLAG & 782350.5 & 3325847.8 & 900420.01741 & 300152.08836 & 3679881.0 & 559011.1 \\
\hline FLAG & 782350.7 & 3325844.6 & 900420.01301 & 300151.98436 & 3679881.5 & 559000.6 \\
\hline FLAG & 782438.2 & 3325913.1 & 900416.68485 & 300154.13423 & 3680171.7 & 559221.0 \\
\hline FLAG & 782470.3 & 3325923.8 & 900415.47766 & 300154.45473 & 3680277.4 & 559254.6 \\
\hline
\end{tabular}




\begin{tabular}{|c|c|c|c|c|c|c|}
\hline \multirow[b]{2}{*}{ Comment } & \multicolumn{2}{|c|}{ UTM (Zone 15) } & \multicolumn{2}{|c|}{ Geographic } & \multicolumn{2}{|c|}{ State Plane, LA South } \\
\hline & $\mathrm{X}, \mathrm{m}$ & $\mathrm{Y}, \mathrm{m}$ & Longitude & Latitude & X, U.S. ft & Y, U.S. ft \\
\hline FLAG & 782478.0 & 3325926.6 & 900415.18787 & 300154.53918 & 3680302.8 & 559263.4 \\
\hline FLAG & 782491.0 & 3325930.3 & 900414.69958 & 300154.64843 & 3680345.6 & 559274.9 \\
\hline FLAG & 782492.5 & 3325921.6 & 900414.65195 & 300154.36490 & 3680350.1 & 559246.3 \\
\hline FLAG & 782493.8 & 3325916.9 & 900414.60796 & 300154.21132 & 3680354.1 & 559230.8 \\
\hline FLAG & 782494.2 & 3325910.8 & 900414.59887 & 300154.01307 & 3680355.1 & 559210.8 \\
\hline FLAG & 782515.8 & 3325931.2 & 900413.77395 & 300154.65702 & 3680426.9 & 559276.6 \\
\hline FLAG & 782529.3 & 3325947.7 & 900413.25479 & 300155.18116 & 3680472.0 & 559330.1 \\
\hline FLAG & 782531.3 & 3325938.1 & 900413.18939 & 300154.86801 & 3680478.1 & 559298.5 \\
\hline FLAG & 782538.7 & 3325944.9 & 900412.90695 & 300155.08250 & 3680502.7 & 559320.5 \\
\hline FLAG & 782554.9 & 3325933.2 & 900412.31405 & 300154.68942 & 3680555.2 & 559281.3 \\
\hline FLAG & 782574.4 & 3325956.5 & 900411.56466 & 300155.42920 & 3680620.2 & 559356.8 \\
\hline FLAG & 782591.7 & 3325961.0 & 900410.91526 & 300155.56082 & 3680677.2 & 559370.7 \\
\hline FLAG & 782602.9 & 3325922.2 & 900410.53469 & 300154.29262 & 3680712.0 & 559243.0 \\
\hline FLAG & 782603.0 & 3325965.4 & 900410.48969 & 300155.69419 & 3680714.4 & 559384.6 \\
\hline FLAG & 782608.0 & 3325962.2 & 900410.30630 & 300155.58621 & 3680730.7 & 559373.9 \\
\hline FLAG & 782614.7 & 3325968.9 & 900410.05007 & 300155.79802 & 3680753.0 & 559395.5 \\
\hline FLAG & 782616.3 & 3325959.2 & 900409.99967 & 300155.48197 & 3680757.7 & 559363.6 \\
\hline FLAG & 782618.1 & 3325938.3 & 900409.95252 & 300154.80236 & 3680762.6 & 559295.0 \\
\hline FLAG & 782619.9 & 3325936.2 & 900409.88741 & 300154.73272 & 3680768.4 & 559288.1 \\
\hline FLAG & 782621.3 & 3325930.2 & 900409.84093 & 300154.53689 & 3680772.7 & 559268.3 \\
\hline FLAG & 782637.6 & 3325975.5 & 900409.18984 & 300155.99313 & 3680828.3 & 559416.0 \\
\hline FLAG & 782658.8 & 3325982.0 & 900408.39311 & 300156.18640 & 3680898.2 & 559436.3 \\
\hline FLAG & 782666.1 & 3325984.9 & 900408.11813 & 300156.27442 & 3680922.2 & 559445.5 \\
\hline FLAG & 782673.0 & 3325957.5 & 900407.88701 & 300155.37967 & 3680943.5 & 559355.3 \\
\hline FLAG & 782673.4 & 3325959.9 & 900407.86981 & 300155.45721 & 3680945.0 & 559363.2 \\
\hline FLAG & 782677.0 & 3325994.5 & 900407.70250 & 300156.57684 & 3680958.4 & 559476.5 \\
\hline FLAG & 782683.1 & 3325989.9 & 900407.47943 & 300156.42252 & 3680978.2 & 559461.1 \\
\hline FLAG & 782699.5 & 3325995.6 & 900406.86245 & 300156.59382 & 3681032.2 & 559479.0 \\
\hline FLAG & 782706.2 & 3326014.7 & 900406.59436 & 300157.20796 & 3681055.1 & 559541.3 \\
\hline FLAG & 782712.3 & 3325998.2 & 900406.38266 & 300156.66754 & 3681074.3 & 559486.9 \\
\hline FLAG & 782714.9 & 3326014.4 & 900406.27023 & 300157.19100 & 3681083.6 & 559539.9 \\
\hline FLAG & 782717.3 & 3326000.8 & 900406.19373 & 300156.74774 & 3681090.8 & 559495.2 \\
\hline FLAG & 782720.8 & 3326014.5 & 900406.05013 & 300157.18933 & 3681103.0 & 559539.9 \\
\hline FLAG & 782724.3 & 3326003.6 & 900405.93003 & 300156.83276 & 3681113.9 & 559504.0 \\
\hline FLAG & 782724.9 & 3326013.6 & 900405.89810 & 300157.15672 & 3681116.4 & 559536.8 \\
\hline FLAG & 782728.4 & 3326011.7 & 900405.76941 & 300157.09217 & 3681127.7 & 559530.4 \\
\hline FLAG & 782731.6 & 3326005.6 & 900405.65591 & 300156.89158 & 3681137.9 & 559510.2 \\
\hline FLAG & 782734.1 & 3326001.8 & 900405.56632 & 300156.76621 & 3681146.0 & 559497.7 \\
\hline FLAG & 782743.1 & 3326009.1 & 900405.22374 & 300156.99558 & 3681175.8 & 559521.2 \\
\hline FLAG & 782766.7 & 3326016.2 & 900404.33693 & 300157.20632 & 3681253.5 & 559543.3 \\
\hline
\end{tabular}




\begin{tabular}{|c|c|c|c|c|c|c|}
\hline \multirow[b]{2}{*}{ Comment } & \multicolumn{2}{|c|}{ UTM (Zone 15) } & \multicolumn{2}{|c|}{ Geographic } & \multicolumn{2}{|c|}{ State Plane, LA South } \\
\hline & $\mathrm{X}, \mathrm{m}$ & $\mathrm{Y}, \mathrm{m}$ & Longitude & Latitude & X, U.S. ft & Y, U.S. ft \\
\hline FLAG & 782773.4 & 3326018.6 & 900404.08480 & 300157.27861 & 3681275.6 & 559550.8 \\
\hline FLAG & 782779.4 & 3326020.7 & 900403.85906 & 300157.34176 & 3681295.4 & 559557.4 \\
\hline FLAG & 782782.8 & 3325994.9 & 900403.75694 & 300156.50183 & 3681305.3 & 559472.7 \\
\hline FLAG & 782786.0 & 3326022.2 & 900403.61152 & 300157.38494 & 3681317.1 & 559562.0 \\
\hline FLAG & 782790.9 & 3326022.7 & 900403.42832 & 300157.39708 & 3681333.2 & 559563.5 \\
\hline FLAG & 782800.3 & 3326025.0 & 900403.07561 & 300157.46389 & 3681364.1 & 559570.5 \\
\hline FLAG & 782811.5 & 3326027.4 & 900402.65568 & 300157.53244 & 3681400.9 & 559577.9 \\
\hline FLAG & 782816.3 & 3326026.2 & 900402.47784 & 300157.48952 & 3681416.6 & 559573.7 \\
\hline FLAG & 782821.8 & 3326023.5 & 900402.27533 & 300157.39734 & 3681434.5 & 559564.6 \\
\hline FLAG & 782835.8 & 3326018.5 & 900401.75806 & 300157.22346 & 3681480.2 & 559547.5 \\
\hline FLAG & 782850.8 & 3326013.6 & 900401.20341 & 300157.05200 & 3681529.1 & 559530.8 \\
\hline FLAG & 782868.9 & 3326006.0 & 900400.53575 & 300156.79035 & 3681588.1 & 559505.0 \\
\hline FLAG & 782873.9 & 3325964.4 & 900400.38908 & 300155.43645 & 3681602.5 & 559368.4 \\
\hline FLAG & 782892.6 & 3325965.9 & 900359.69035 & 300155.46956 & 3681663.9 & 559372.4 \\
\hline FLAG & 782909.9 & 3325960.2 & 900359.05070 & 300155.27022 & 3681720.3 & 559352.9 \\
\hline FLAG & 782962.0 & 3325970.6 & 900357.09801 & 300155.56429 & 3681891.6 & 559384.5 \\
\hline FLAG & 782968.8 & 3325968.9 & 900356.84607 & 300155.50348 & 3681913.8 & 559378.6 \\
\hline FLAG & 782973.4 & 3325968.1 & 900356.67531 & 300155.47369 & 3681928.8 & 559375.7 \\
\hline FLAG & 782976.3 & 3325966.6 & 900356.56860 & 300155.42261 & 3681938.3 & 559370.7 \\
\hline FLAG & 782982.3 & 3325964.1 & 900356.34726 & 300155.33650 & 3681957.8 & 559362.2 \\
\hline FLAG & 782990.8 & 3325960.2 & 900356.03404 & 300155.20289 & 3681985.5 & 559349.0 \\
\hline FLAG & 783000.7 & 3325959.5 & 900355.66555 & 300155.17193 & 3682017.9 & 559346.2 \\
\hline FLAG & 783007.2 & 3325961.5 & 900355.42126 & 300155.23141 & 3682039.3 & 559352.5 \\
\hline FLAG & 783013.2 & 3325961.2 & 900355.19781 & 300155.21669 & 3682059.0 & 559351.2 \\
\hline FLAG & 783031.2 & 3325950.8 & 900354.53657 & 300154.86427 & 3682117.5 & 559316.3 \\
\hline FLAG & 783032.2 & 3326024.7 & 900354.42857 & 300157.26117 & 3682124.3 & 559558.5 \\
\hline FLAG & 783038.1 & 3326024.8 & 900354.20847 & 300157.25950 & 3682143.7 & 559558.5 \\
\hline FLAG & 783038.2 & 3325951.1 & 900354.27526 & 300154.86817 & 3682140.5 & 559316.9 \\
\hline FLAG & 783040.5 & 3325951.0 & 900354.18959 & 300154.86301 & 3682148.0 & 559316.5 \\
\hline FLAG & 783044.2 & 3325951.3 & 900354.05134 & 300154.86967 & 3682160.1 & 559317.3 \\
\hline FLAG & 783045.6 & 3326022.3 & 900353.93119 & 300157.17214 & 3682168.1 & 559550.0 \\
\hline FLAG & 783048.8 & 3325951.2 & 900353.87990 & 300154.86259 & 3682175.2 & 559316.7 \\
\hline FLAG & 783050.5 & 3325951.2 & 900353.81651 & 300154.86118 & 3682180.8 & 559316.6 \\
\hline FLAG & 783056.3 & 3325958.1 & 900353.59363 & 300155.08022 & 3682200.1 & 559339.0 \\
\hline FLAG & 783059.1 & 3326020.9 & 900353.42913 & 300157.11548 & 3682212.3 & 559544.7 \\
\hline FLAG & 783062.6 & 3325965.5 & 900353.35163 & 300155.31508 & 3682221.1 & 559362.9 \\
\hline FLAG & 783063.5 & 3326020.0 & 900353.26592 & 300157.08261 & 3682226.7 & 559541.6 \\
\hline FLAG & 783064.1 & 3325955.2 & 900353.30556 & 300154.97964 & 3682225.6 & 559329.1 \\
\hline FLAG & 783064.7 & 3325968.3 & 900353.27065 & 300155.40417 & 3682228.2 & 559372.0 \\
\hline FLAG & 783066.9 & 3325966.1 & 900353.19072 & 300155.33096 & 3682235.3 & 559364.7 \\
\hline
\end{tabular}




\begin{tabular}{|c|c|c|c|c|c|c|}
\hline \multirow[b]{2}{*}{ Comment } & \multicolumn{2}{|c|}{ UTM (Zone 15) } & \multicolumn{2}{|c|}{ Geographic } & \multicolumn{2}{|c|}{ State Plane, LA South } \\
\hline & $\mathrm{X}, \mathrm{m}$ & $\mathrm{Y}, \mathrm{m}$ & Longitude & Latitude & X, U.S. ft & Y, U.S. ft \\
\hline FLAG & 783067.3 & 3326031.7 & 900353.11303 & 300157.45906 & 3682239.7 & 559579.7 \\
\hline FLAG & 783072.4 & 3326028.5 & 900352.92592 & 300157.35099 & 3682256.3 & 559569.0 \\
\hline FLAG & 783075.2 & 3326028.2 & 900352.82180 & 300157.33893 & 3682265.5 & 559567.9 \\
\hline FLAG & 783105.2 & 3326007.5 & 900351.72294 & 300156.64232 & 3682362.8 & 559498.6 \\
\hline FLAG & 783114.4 & 3326027.5 & 900351.36074 & 300157.28357 & 3682393.9 & 559563.7 \\
\hline FLAG & 783135.5 & 3326028.7 & 900350.57279 & 300157.30493 & 3682463.2 & 559566.6 \\
\hline FLAG & 783136.5 & 3326054.7 & 900350.51062 & 300158.14769 & 3682467.7 & 559651.8 \\
\hline FLAG & 783139.8 & 3326053.4 & 900350.38881 & 300158.10276 & 3682478.4 & 559647.4 \\
\hline FLAG & 783143.1 & 3326055.1 & 900350.26413 & 300158.15517 & 3682489.3 & 559652.8 \\
\hline FLAG & 783146.6 & 3326054.6 & 900350.13409 & 300158.13603 & 3682500.8 & 559651.0 \\
\hline FLAG & 783148.8 & 3326053.9 & 900350.05273 & 300158.11149 & 3682508.0 & 559648.6 \\
\hline FLAG & 783165.9 & 3326010.9 & 900349.45625 & 300156.70208 & 3682562.0 & 559506.8 \\
\hline FLAG & 783166.9 & 3326015.4 & 900349.41465 & 300156.84725 & 3682565.5 & 559521.5 \\
\hline FLAG & 783201.5 & 3326080.2 & 900348.06242 & 300158.92091 & 3682682.0 & 559732.3 \\
\hline FLAG & 783201.8 & 3326073.9 & 900348.05726 & 300158.71625 & 3682682.7 & 559711.7 \\
\hline FLAG & 783202.7 & 3326067.4 & 900348.02993 & 300158.50461 & 3682685.3 & 559690.3 \\
\hline FLAG & 783202.7 & 3326069.8 & 900348.02763 & 300158.58247 & 3682685.4 & 559698.2 \\
\hline FLAG & 783256.9 & 3326064.0 & 900346.01212 & 300158.34913 & 3682862.8 & 559676.6 \\
\hline FLAG & 783258.7 & 3326064.3 & 900345.94471 & 300158.35737 & 3682868.7 & 559677.5 \\
\hline FLAG & 783261.3 & 3326064.9 & 900345.84718 & 300158.37467 & 3682877.3 & 559679.3 \\
\hline FLAG & 783266.6 & 3326065.7 & 900345.64878 & 300158.39621 & 3682894.7 & 559681.7 \\
\hline FLAG & 783269.7 & 3326065.9 & 900345.53300 & 300158.40012 & 3682904.9 & 559682.2 \\
\hline FLAG & 783293.9 & 3326006.9 & 900344.68711 & 300156.46566 & 3682981.4 & 559487.6 \\
\hline FLAG & 783293.9 & 3326010.7 & 900344.68347 & 300156.58895 & 3682981.6 & 559500.1 \\
\hline FLAG & 783294.3 & 3326002.5 & 900344.67641 & 300156.32257 & 3682982.5 & 559473.2 \\
\hline FLAG & 783294.5 & 3326005.0 & 900344.66655 & 300156.40351 & 3682983.3 & 559481.3 \\
\hline FLAG & 783298.3 & 3326050.7 & 900344.48109 & 300157.88311 & 3682997.9 & 559631.0 \\
\hline FLAG & 783304.5 & 3326051.9 & 900344.24875 & 300157.91688 & 3683018.3 & 559634.6 \\
\hline FLAG & 783331.8 & 3326079.7 & 900343.20413 & 300158.79611 & 3683109.1 & 559724.5 \\
\hline FLAG & 783332.7 & 3326056.1 & 900343.19318 & 300158.02965 & 3683111.0 & 559647.0 \\
\hline FLAG & 783351.5 & 3326083.3 & 900342.46609 & 300158.89650 & 3683173.9 & 559735.3 \\
\hline FLAG & 783357.1 & 3326084.3 & 900342.25631 & 300158.92428 & 3683192.3 & 559738.3 \\
\hline FLAG & 783363.6 & 3326062.5 & 900342.03482 & 300158.21155 & 3683212.6 & 559666.5 \\
\hline FLAG & 783365.6 & 3326083.2 & 900341.94041 & 300158.88150 & 3683220.1 & 559734.3 \\
\hline FLAG & 783487.2 & 3326077.5 & 900337.41153 & 300158.59518 & 3683618.5 & 559709.8 \\
\hline FLAG & 783487.6 & 3326071.4 & 900337.40246 & 300158.39693 & 3683619.5 & 559689.8 \\
\hline FLAG & 783487.8 & 3326094.6 & 900337.37277 & 300159.14950 & 3683621.3 & 559765.8 \\
\hline FLAG & 783640.6 & 3326099.8 & 900331.67003 & 300159.19076 & 3684122.4 & 559775.6 \\
\hline FLAG & 783647.5 & 3326102.6 & 900331.41005 & 300159.27585 & 3684145.2 & 559784.4 \\
\hline FLAG & 783660.8 & 3326093.0 & 900330.92331 & 300158.95328 & 3684188.3 & 559752.3 \\
\hline
\end{tabular}




\begin{tabular}{|c|c|c|c|c|c|c|}
\hline \multirow[b]{2}{*}{ Comment } & \multicolumn{2}{|c|}{ UTM (Zone 15) } & \multicolumn{2}{|c|}{ Geographic } & \multicolumn{2}{|c|}{ State Plane, LA South } \\
\hline & $\mathrm{X}, \mathrm{m}$ & $\mathrm{Y}, \mathrm{m}$ & Longitude & Latitude & X, U.S. ft & Y, U.S. ft \\
\hline FLAG & 783704.7 & 3326059.5 & 900329.31846 & 300157.82972 & 3684330.6 & 559640.4 \\
\hline FLAG & 783705.0 & 3326056.4 & 900329.31024 & 300157.72889 & 3684331.5 & 559630.2 \\
\hline FLAG & 783707.5 & 3326054.2 & 900329.21913 & 300157.65543 & 3684339.6 & 559622.9 \\
\hline FLAG & 783707.9 & 3326063.2 & 900329.19558 & 300157.94710 & 3684341.3 & 559652.4 \\
\hline FLAG & 783712.5 & 3326066.0 & 900329.02137 & 300158.03411 & 3684356.5 & 559661.3 \\
\hline FLAG & 783719.4 & 3326054.8 & 900328.77482 & 300157.66496 & 3684378.6 & 559624.3 \\
\hline FLAG & 783725.9 & 3326052.4 & 900328.53474 & 300157.58167 & 3684399.8 & 559616.1 \\
\hline FLAG & 783730.8 & 3326052.4 & 900328.35203 & 300157.57758 & 3684415.9 & 559615.9 \\
\hline FLAG & 783750.7 & 3326057.8 & 900327.60480 & 300157.73618 & 3684481.4 & 559632.6 \\
\hline FLAG & 783753.1 & 3326058.4 & 900327.51473 & 300157.75364 & 3684489.3 & 559634.5 \\
\hline FLAG & 783755.0 & 3326059.2 & 900327.44312 & 300157.77801 & 3684495.5 & 559637.0 \\
\hline FLAG & 783756.7 & 3326059.8 & 900327.37915 & 300157.79606 & 3684501.1 & 559638.9 \\
\hline FLAG & 783764.9 & 3326078.3 & 900327.05564 & 300158.38945 & 3684528.9 & 559699.1 \\
\hline FLAG & 783970.8 & 3326074.0 & 900319.38200 & 300158.07801 & 3685203.7 & 559675.2 \\
\hline FLAG & 783976.4 & 3326073.8 & 900319.17338 & 300158.06684 & 3685222.0 & 559674.3 \\
\hline FLAG & 783992.1 & 3326142.1 & 900318.52237 & 300200.26975 & 3685276.8 & 559897.4 \\
\hline FLAG & 783993.1 & 3326126.8 & 900318.49977 & 300159.77250 & 3685279.3 & 559847.2 \\
\hline FLAG & 784022.5 & 3326203.8 & 900317.32954 & 300202.24623 & 3685379.4 & 560098.3 \\
\hline FLAG & 784022.8 & 3326202.7 & 900317.31941 & 300202.21029 & 3685380.3 & 560094.6 \\
\hline FLAG & 784023.1 & 3326201.2 & 900317.30966 & 300202.16137 & 3685381.2 & 560089.7 \\
\hline FLAG & 784024.5 & 3326186.1 & 900317.27196 & 300201.67028 & 3685385.1 & 560040.1 \\
\hline FLAG & 784025.2 & 3326183.2 & 900317.24864 & 300201.57560 & 3685387.3 & 560030.6 \\
\hline FLAG & 784025.4 & 3326181.0 & 900317.24330 & 300201.50405 & 3685387.8 & 560023.4 \\
\hline FLAG & 784025.9 & 3326176.2 & 900317.22926 & 300201.34790 & 3685389.2 & 560007.6 \\
\hline FLAG & 784028.7 & 3326141.7 & 900317.15798 & 300200.22619 & 3685396.7 & 559894.4 \\
\hline FLAG & 784029.1 & 3326143.4 & 900317.14143 & 300200.28102 & 3685398.1 & 559899.9 \\
\hline FLAG & 784029.5 & 3326145.2 & 900317.12479 & 300200.33908 & 3685399.5 & 559905.8 \\
\hline FLAG & 784034.6 & 3326150.0 & 900316.93000 & 300200.49056 & 3685416.5 & 559921.3 \\
\hline FLAG & 784051.3 & 3326146.5 & 900316.31064 & 300200.36305 & 3685471.1 & 559909.0 \\
\hline FLAG & 784051.6 & 3326143.0 & 900316.30281 & 300200.24924 & 3685471.9 & 559897.5 \\
\hline FLAG & 784052.0 & 3326141.3 & 900316.28953 & 300200.19375 & 3685473.1 & 559892.0 \\
\hline FLAG & 784203.6 & 3326207.2 & 900310.57322 & 300202.20519 & 3685973.2 & 560100.7 \\
\hline FLAG & 784205.2 & 3326207.1 & 900310.51365 & 300202.20060 & 3685978.5 & 560100.3 \\
\hline FLAG & 784205.3 & 3326183.4 & 900310.53269 & 300201.43157 & 3685977.7 & 560022.6 \\
\hline FLAG & 784206.4 & 3326183.4 & 900310.49168 & 300201.43065 & 3685981.3 & 560022.6 \\
\hline FLAG & 784217.0 & 3326217.6 & 900310.06355 & 300202.53142 & 3686017.7 & 560134.2 \\
\hline FLAG & 784219.8 & 3326217.9 & 900309.95885 & 300202.53881 & 3686026.9 & 560135.0 \\
\hline FLAG & 784220.0 & 3326218.0 & 900309.95130 & 300202.54189 & 3686027.5 & 560135.4 \\
\hline FLAG & 784243.7 & 3326218.5 & 900309.06707 & 300202.53829 & 3686105.2 & 560135.9 \\
\hline FLAG & 784252.6 & 3326221.3 & 900308.73250 & 300202.62170 & 3686134.6 & 560144.6 \\
\hline
\end{tabular}




\begin{tabular}{|c|c|c|c|c|c|c|}
\hline \multirow[b]{2}{*}{ Comment } & \multicolumn{2}{|c|}{ UTM (Zone 15) } & \multicolumn{2}{|c|}{ Geographic } & \multicolumn{2}{|c|}{ State Plane, LA South } \\
\hline & $\mathrm{X}, \mathrm{m}$ & $\mathrm{Y}, \mathrm{m}$ & Longitude & Latitude & X, U.S. ft & Y, U.S. ft \\
\hline FLAG & 784778.6 & 3326233.0 & 900249.10723 & 300202.56107 & 3687859.5 & 560157.8 \\
\hline FLAG & 784778.7 & 3326235.1 & 900249.10148 & 300202.62912 & 3687859.9 & 560164.7 \\
\hline FLAG & 784785.9 & 3326270.6 & 900248.79882 & 300203.77489 & 3687885.2 & 560280.7 \\
\hline FLAG & 784787.4 & 3326267.2 & 900248.74616 & 300203.66332 & 3687890.0 & 560269.5 \\
\hline FLAG & 784800.3 & 3326234.5 & 900248.29661 & 300202.59156 & 3687930.7 & 560161.7 \\
\hline FLAG & 784802.5 & 3326237.0 & 900248.21217 & 300202.67083 & 3687938.0 & 560169.8 \\
\hline FLAG & 784833.8 & 3326246.8 & 900247.03559 & 300202.96257 & 3688041.1 & 560200.4 \\
\hline FLAG & 784834.5 & 3326244.6 & 900247.01161 & 300202.89060 & 3688043.3 & 560193.2 \\
\hline FLAG & 784835.4 & 3326241.1 & 900246.98142 & 300202.77629 & 3688046.1 & 560181.7 \\
\hline FLAG & 784851.5 & 3326278.9 & 900246.34466 & 300203.98922 & 3688100.7 & 560304.8 \\
\hline FLAG & 784863.3 & 3326255.3 & 900245.92738 & 300203.21363 & 3688138.2 & 560226.9 \\
\hline FLAG & 784863.7 & 3326251.9 & 900245.91574 & 300203.10298 & 3688139.4 & 560215.7 \\
\hline FLAG & 784864.2 & 3326249.5 & 900245.89941 & 300203.02469 & 3688140.9 & 560207.8 \\
\hline FLAG & 784866.5 & 3326282.6 & 900245.78176 & 300204.09670 & 3688150.0 & 560316.2 \\
\hline FLAG & 784950.0 & 3326272.1 & 900242.67825 & 300203.68604 & 3688423.3 & 560277.8 \\
\hline FLAG & 784951.0 & 3326265.9 & 900242.64693 & 300203.48405 & 3688426.2 & 560257.4 \\
\hline FLAG & 784951.4 & 3326262.5 & 900242.63529 & 300203.37340 & 3688427.4 & 560246.3 \\
\hline FLAG & 784951.8 & 3326259.9 & 900242.62288 & 300203.28871 & 3688428.6 & 560237.7 \\
\hline FLAG & 784951.9 & 3326257.2 & 900242.62175 & 300203.20102 & 3688428.8 & 560228.9 \\
\hline FLAG & 785049.2 & 3326303.7 & 900238.94873 & 300204.62814 & 3688750.0 & 560376.6 \\
\hline FLAG & 785058.9 & 3326300.9 & 900238.58972 & 300204.52916 & 3688781.6 & 560367.0 \\
\hline FLAG & 785068.1 & 3326298.9 & 900238.24859 & 300204.45655 & 3688811.7 & 560360.0 \\
\hline FLAG & 785096.8 & 3326249.5 & 900237.22601 & 300202.82970 & 3688903.4 & 560196.7 \\
\hline FLAG & 785098.2 & 3326254.2 & 900237.16928 & 300202.98102 & 3688908.2 & 560212.0 \\
\hline FLAG & 785099.5 & 3326259.0 & 900237.11618 & 300203.13567 & 3688912.7 & 560227.7 \\
\hline FLAG & 785102.1 & 3326265.6 & 900237.01286 & 300203.34762 & 3688921.6 & 560249.2 \\
\hline FLAG & 785103.2 & 3326269.9 & 900236.96770 & 300203.48621 & 3688925.4 & 560263.3 \\
\hline FLAG & 785164.7 & 3326251.0 & 900234.69266 & 300202.82142 & 3689126.1 & 560198.4 \\
\hline FLAG & 785175.3 & 3326282.5 & 900234.26702 & 300203.83454 & 3689162.3 & 560301.1 \\
\hline FLAG & 785185.3 & 3326267.8 & 900233.90831 & 300203.34921 & 3689194.4 & 560252.5 \\
\hline FLAG & 785213.1 & 3326191.7 & 900232.94506 & 300200.85683 & 3689281.9 & 560001.6 \\
\hline FLAG & 785215.0 & 3326191.1 & 900232.87479 & 300200.83576 & 3689288.1 & 559999.6 \\
\hline FLAG & 785216.4 & 3326164.2 & 900232.84852 & 300159.96182 & 3689291.4 & 559911.3 \\
\hline FLAG & 785217.6 & 3326164.9 & 900232.80310 & 300159.98352 & 3689295.4 & 559913.6 \\
\hline FLAG & 785219.2 & 3326189.9 & 900232.71933 & 300200.79331 & 3689301.8 & 559995.5 \\
\hline FLAG & 785219.3 & 3326190.0 & 900232.71551 & 300200.79647 & 3689302.2 & 559995.8 \\
\hline FLAG & 785219.9 & 3326186.9 & 900232.69612 & 300200.69538 & 3689304.0 & 559985.6 \\
\hline FLAG & 785220.2 & 3326185.2 & 900232.68658 & 300200.63998 & 3689304.9 & 559980.0 \\
\hline FLAG & 785220.3 & 3326183.6 & 900232.68439 & 300200.58798 & 3689305.1 & 559974.7 \\
\hline FLAG & 785222.1 & 3326165.3 & 900232.63492 & 300159.99273 & 3689310.2 & 559914.7 \\
\hline
\end{tabular}




\begin{tabular}{|c|c|c|c|c|c|c|}
\hline \multirow[b]{2}{*}{ Comment } & \multicolumn{2}{|c|}{ UTM (Zone 15) } & \multicolumn{2}{|c|}{ Geographic } & \multicolumn{2}{|c|}{ State Plane, LA South } \\
\hline & $\mathrm{X}, \mathrm{m}$ & $\mathrm{Y}, \mathrm{m}$ & Longitude & Latitude & X, U.S. $\mathrm{ft}$ & Y, U.S. ft \\
\hline FLAG & 3522 & 3326 & 00232 & 300159 & 3689318.9 & 559903.7 \\
\hline FLAG & 35225.6 & 3326165.9 & 900232.50383 & 300200.00926 & 3689321.7 & 559916.5 \\
\hline FLAG & 35226.1 & 3326168.9 & 900232.48229 & 300200.10617 & 3689323.4 & 559926.3 \\
\hline FLAG & 85226.3 & 3326171.8 & 900232.47204 & 300200.20009 & 3689324.2 & 559935.8 \\
\hline FLAG & 785226.6 & 3326175.2 & 900232.45757 & 300200.31016 & 3689325.4 & 559946.9 \\
\hline FLAG & 785227.6 & 3326178.3 & 900232.41729 & 300200.40990 & 3689328.8 & 57.0 \\
\hline FLAG & 785227.7 & 3326181.1 & 900232.41087 & 300200.50066 & 3689329.3 & 559966.2 \\
\hline FLAG & 785228.0 & 3326184.2 & 900232.39669 & 300200.60099 & 3689330.4 & 559976.3 \\
\hline FLAG & 785228.4 & 3326190.4 & 900232.37580 & 300200.80181 & 3689332.0 & 559996.7 \\
\hline FLAG & 785228.7 & 3326187.4 & 900232.36750 & 300200.70422 & 3689332.9 & 559986.8 \\
\hline FLAG & 785228.7 & 3326192.2 & 900232.36287 & 300200.85996 & 3689333.1 & 560002.5 \\
\hline FLAG & 785229.7 & 3326195.6 & 900232.32231 & 300200.96943 & 3689336.5 & 560013.6 \\
\hline FLAG & 785233.5 & 3326208.2 & 900232.16846 & 300201.37505 & 3689349.6 & 54.8 \\
\hline FLAG & 7852 & 3326213.2 & 15991 & 719 & 0.2 & 560 \\
\hline FLAG & 1.2 & 326179.5 & 003 & 3002 & 368 & 5 \\
\hline FLAG & 8 & 3326 & 1179 & 300 & 3689 & 560082.6 \\
\hline FLAG & 785237.8 & 3326179.0 & 900232.03628 & 300200.42405 & 3689362.3 & 559958.8 \\
\hline FLAG & 785238.7 & 3326224.1 & 900231.95923 & 300201.88656 & 3689367.4 & 560106.6 \\
\hline FLAG & 785241.3 & 3326228.0 & 900231.85851 & 300202.01092 & 3689376.1 & 560119.3 \\
\hline FLAG & 785242.1 & 3326178.6 & 900231.87632 & 300200.40746 & 3689376.4 & 559957.3 \\
\hline FLAG & 785248.8 & 3326177.7 & 900231.62736 & 300200.37264 & 3689398.3 & 559954.0 \\
\hline FLAG & 785253.5 & 3326202.0 & 900231.42867 & 300201.15711 & 3689414.9 & 560033.5 \\
\hline FLAG & 8 & .2 & 90 & 22 & 1.7 & 52.2 \\
\hline FLAG & 4 & 4.2 & 90 & 0 & 3689420.2 & 559975.0 \\
\hline FLAG & 785255.4 & 3326206.8 & 900231.35319 & 300201.31125 & 3689421.3 & 560049.1 \\
\hline FLAG & 785255.6 & 3326190.4 & 900231.36155 & 300200.77899 & 3689421.2 & 559995.4 \\
\hline FLAG & 785255.8 & 3326187.9 & 900231.35650 & 300200.69771 & 3689421.7 & 559987.1 \\
\hline FLAG & 785257.0 & 3326176.7 & 900231.32255 & 300200.33332 & 3689425.1 & 559950.4 \\
\hline FLAG & 785258.7 & 3326172.5 & 900231.26321 & 300200.19562 & 3689430.5 & 559936.5 \\
\hline FLAG & 785260.1 & 3326169.3 & 900231.21410 & 300200.09062 & 3689434.9 & 559926.0 \\
\hline FLAG & 785260.6 & 3326166.7 & 900231.19796 & 300200.00585 & 3689436.4 & 559917.4 \\
\hline FLAG & 785260.8 & 3326163.8 & 900231.19330 & 300159.91159 & 3689437.0 & 559907.9 \\
\hline FLAG & 785261.0 & 3326161.2 & 900231.18835 & 300159.82706 & 3689437.5 & 559899.4 \\
\hline FLAG & 85261.2 & 326155.9 & 900231.18600 & 300159.65494 & 3689437.9 & 559882.0 \\
\hline FLAG & 785302.1 & 3326197.3 & 900229.62097 & 300200.96384 & 3689574.0 & 560015.7 \\
\hline FLAG & 7853 & 3326 & 900 & 300 & 37.7 & 5600 \\
\hline FLAG & 78 & 33261 & 900 & 30020 & 3689602.4 & 5600 \\
\hline FLAG & 785315.4 & 3326150.5 & 900229.17018 & 300159.43425 & 3689615.3 & 559861.7 \\
\hline FLAG & 785316.5 & 3326193.6 & 900229.08759 & 300200.83170 & 3689621.0 & 560002.9 \\
\hline FLAG & 785318.9 & 3326151.2 & 900229.03899 & 300159.45402 & 3689626.8 & 559863.8 \\
\hline
\end{tabular}




\begin{tabular}{|c|c|c|c|c|c|c|}
\hline \multirow[b]{2}{*}{ Comment } & \multicolumn{2}{|c|}{ UTM (Zone 15) } & \multicolumn{2}{|c|}{ Geographic } & \multicolumn{2}{|c|}{ State Plane, LA South } \\
\hline & $\mathrm{X}, \mathrm{m}$ & $\mathrm{Y}, \mathrm{m}$ & Longitude & Latitude & X, U.S. ft & Y, U.S. ft \\
\hline FLAG & 785321.4 & 3326151.1 & 900228.94587 & 300159.44868 & 3689635.0 & 559863.4 \\
\hline FLAG & 785322.9 & 3326152.4 & 900228.88868 & 300159.48960 & 3689640.0 & 559867.6 \\
\hline FLAG & 785326.5 & 3326152.6 & 900228.75425 & 300159.49307 & 3689651.8 & 559868.0 \\
\hline FLAG & 785366.1 & 3326150.8 & 900227.27937 & 300159.40143 & 3689781.5 & 559860.2 \\
\hline FLAG & 785366.3 & 3326148.0 & 900227.27461 & 300159.31041 & 3689782.1 & 559851.1 \\
\hline FLAG & 785379.8 & 3326106.1 & 900226.81164 & 300157.93964 & 3689824.3 & 559713.1 \\
\hline FLAG & 785385.7 & 3326104.3 & 900226.59338 & 300157.87628 & 3689843.6 & 559706.9 \\
\hline FLAG & 785387.3 & 3326020.1 & 900226.61495 & 300155.14308 & 3689844.8 & 559430.8 \\
\hline FLAG & 785389.1 & 3326021.2 & 900226.54677 & 300155.17726 & 3689850.7 & 559434.3 \\
\hline FLAG & 785391.9 & 3326023.2 & 900226.44043 & 300155.23979 & 3689860.0 & 559440.7 \\
\hline FLAG & 785393.9 & 3326025.0 & 900226.36412 & 300155.29652 & 3689866.7 & 559446.5 \\
\hline FLAG & 785400.8 & 3326030.4 & 900226.10162 & 300155.46593 & 3689889.5 & 559463.9 \\
\hline FLAG & 785403.9 & 3326033.7 & 900225.98285 & 300155.57039 & 3689899.9 & 559474.6 \\
\hline FLAG & 785405.8 & 3326035.4 & 900225.91036 & 300155.62395 & 3689906.2 & 559480.0 \\
\hline FLAG & 785470.8 & 3325939.4 & 900223.57928 & 300152.45467 & 3690114.7 & 559162.2 \\
\hline FLAG & 785472.7 & 3325939.0 & 900223.50882 & 300152.44009 & 3690120.9 & 559160.8 \\
\hline FLAG & 785474.0 & 3325938.7 & 900223.46064 & 300152.42927 & 3690125.1 & 559159.8 \\
\hline FLAG & 785 & 332 & 9002 & 300152.42144 & 3690130.4 & 559159.0 \\
\hline FLAG & \begin{tabular}{|l|}
785477.4 \\
\end{tabular} & 3325938.4 & 900223.33415 & 300152.41668 & 3690136.3 & 559158.6 \\
\hline FLAG & 785485.2 & 3325937.6 & 900223.04408 & 300152.38417 & 3690161.8 & 559155.6 \\
\hline FLAG & 785493.0 & 3325936.8 & 900222.75401 & 300152.35167 & 3690187.3 & 559152.6 \\
\hline FLAG & 785498.2 & 3325936.8 & 900222.56011 & 300152.34730 & 3690204.4 & 559152.4 \\
\hline FLAG & 785501.6 & 3325936.7 & 900222.43343 & 300152.34120 & 3690215.5 & 559151.9 \\
\hline FLAG & 785504.6 & 3325936.3 & 900222.32195 & 300152.32571 & 3690225.3 & 559150.4 \\
\hline FLAG & 785506.8 & 3325935.9 & 900222.24031 & 300152.31088 & 3690232.5 & 559149.0 \\
\hline FLAG & 785509.7 & 3325935.5 & 900222.13256 & 300152.29547 & 3690242.0 & 559147.6 \\
\hline FLAG & 785516.6 & 3325935.8 & 900221.87499 & 300152.29941 & 3690264.6 & 559148.2 \\
\hline FLAG & 785517.4 & 3325930.7 & 900221.85008 & 300152.13327 & 3690267.0 & 559131.5 \\
\hline FLAG & 785519.3 & 3325935.5 & 900221.77460 & 300152.28741 & 3690273.5 & 559147.1 \\
\hline FLAG & 785523.0 & 3325935.3 & 900221.63683 & 300152.27781 & 3690285.6 & 559146.3 \\
\hline FLAG & 785524.9 & 3325889.5 & 900221.61019 & 300150.79024 & 3690289.6 & 558996.0 \\
\hline FLAG & 785525.3 & 3325893.5 & 900221.59141 & 300150.91968 & 3690291.1 & 559009.1 \\
\hline FLAG & 785525.4 & 3325894.0 & 900221.58720 & 300150.93582 & 3690291.5 & 559010.8 \\
\hline FLAG & 785525.5 & 3325896.3 & 900221.58125 & 300151.01036 & 3690291.9 & 559018.3 \\
\hline FLAG & 785527.1 & 3325935.0 & 900221.48424 & 300152.26463 & 3690299.0 & 559145.1 \\
\hline FLAG & 785528.7 & 3325896.4 & 900221.46183 & 300151.01091 & 3690302.4 & 559018.5 \\
\hline FLAG & 785528.8 & 3325894.3 & 900221.46013 & 300150.94270 & 3690302.6 & 559011.6 \\
\hline FLAG & 785531.4 & 3325969.6 & 900221.29051 & 300153.38361 & 3690314.8 & 559258.3 \\
\hline FLAG & 785532.6 & 3325910.7 & 900221.30261 & 300151.47160 & 3690315.9 & 559065.2 \\
\hline FLAG & 785533.0 & 3325912.3 & 900221.28615 & 300151.52318 & 3690317.3 & 559070.4 \\
\hline
\end{tabular}




\begin{tabular}{|c|c|c|c|c|c|c|}
\hline \multirow[b]{2}{*}{ Comment } & \multicolumn{2}{|c|}{ UTM (Zone 15) } & \multicolumn{2}{|c|}{ Geographic } & \multicolumn{2}{|c|}{ State Plane, LA South } \\
\hline & $\mathrm{X}, \mathrm{m}$ & $\mathrm{Y}, \mathrm{m}$ & Longitude & Latitude & $\mathrm{X}$, U.S. $\mathrm{ft}$ & Y, U.S. ft \\
\hline FLAG & 785533.8 & 3325915.1 & 900221.25362 & 300151.61335 & 3690320.0 & 559079.5 \\
\hline FLAG & 785534.0 & 3325918.1 & 900221.24327 & 300151.71052 & 3690320.8 & 559089.4 \\
\hline FLAG & 785534.1 & 3325968.0 & 900221.19137 & 300153.32944 & 3690323.5 & 559252.9 \\
\hline FLAG & 785534.7 & 3325920.0 & 900221.21533 & 300151.77158 & 3690323.2 & 559095.6 \\
\hline FLAG & 785539.0 & 3325965.3 & 900221.01127 & 300153.23772 & 3690339.5 & 559243.9 \\
\hline FLAG & 785540.9 & 3325919.9 & 900220.98424 & 300151.76312 & 3690343.5 & 559094.9 \\
\hline FLAG & 785544.2 & 3325963.7 & 900220.81892 & 300153.18144 & 3690356.4 & 559238.4 \\
\hline FLAG & 785545.0 & 3325920.4 & 900220.83088 & 300151.77590 & 3690357.0 & 559096.4 \\
\hline FLAG & 785551.5 & 3325909.9 & 900220.59865 & 300151.42977 & 3690377.8 & 559061.6 \\
\hline FLAG & 785551.9 & 3325921.4 & 900220.57263 & 300151.80255 & 3690379.7 & 559099.3 \\
\hline FLAG & 785552.0 & 3325918.5 & 900220.57170 & 300151.70838 & 3690379.9 & 559089.8 \\
\hline FLAG & 785552.9 & 3325961.1 & 900220.49702 & 300153.08978 & 3690384.8 & 559229.4 \\
\hline FLAG & 785554.4 & 3325916.6 & 900220.48405 & 300151.64472 & 3690387.6 & 559083.5 \\
\hline FLAG & 785555.8 & 3325922.9 & 900220.42576 & 300151.84794 & 3690392.5 & 559104.1 \\
\hline FLAG & 785555.8 & 3325960.3 & 900220.38966 & 300153.06138 & 3690394.3 & 559226.7 \\
\hline FLAG & 785557.3 & 3325914.3 & 900220.37813 & 300151.56766 & 3690397.0 & 559075.8 \\
\hline FLAG & 785559.4 & 3325923.2 & 900220.29124 & 300151.85465 & 3690404.3 & 559104.9 \\
\hline FLAG & 785561.0 & 3325911.4 & 900220.24297 & 300151.47046 & 3690409.0 & 559066.1 \\
\hline FLAG & 785561.1 & 3325911.3 & 900220.23934 & 300151.46713 & 3690409.3 & 559065.8 \\
\hline FLAG & 785562.3 & 3325923.7 & 900220.18262 & 300151.86844 & 3690413.9 & 559106.4 \\
\hline FLAG & 785562.5 & 3325909.7 & 900220.18868 & 300151.41404 & 3690413.9 & 559060.5 \\
\hline FLAG & 785565.1 & 3325924.5 & 900220.07745 & 300151.89205 & 3690423.1 & 559108.9 \\
\hline FLAG & 785565.8 & 3325935.1 & 900220.04111 & 300152.23537 & 3690425.9 & 559143.6 \\
\hline FLAG & 785566.2 & 3325913.1 & 900220.04743 & 300151.52125 & 3690426.1 & 559071.4 \\
\hline FLAG & 785566.2 & 3325931.0 & 900220.03015 & 300152.10201 & 3690427.0 & 559130.1 \\
\hline FLAG & 785567.0 & 3325928.6 & 900220.00264 & 300152.02347 & 3690429.5 & 559122.2 \\
\hline FLAG & 785567.2 & 3325905.5 & 900220.01748 & 300151.27383 & 3690429.1 & 559046.5 \\
\hline FLAG & 785567.6 & 3325925.8 & 900219.98297 & 300151.93212 & 3690431.3 & 559113.0 \\
\hline FLAG & 785567.7 & 3325926.1 & 900219.97895 & 300151.94177 & 3690431.7 & 559114.0 \\
\hline FLAG & 785568.6 & 3325920.6 & 900219.95070 & 300151.76257 & 3690434.4 & 559095.9 \\
\hline FLAG & 785569.6 & 3325903.9 & 900219.92954 & 300151.21990 & 3690436.9 & 559041.1 \\
\hline FLAG & 785570.2 & 3325917.1 & 900219.89442 & 300151.64767 & 3690439.5 & 559084.4 \\
\hline FLAG & 785570.5 & 3325914.8 & 900219.88546 & 300151.57279 & 3690440.3 & 559076.8 \\
\hline FLAG & 785570.5 & 3325914.9 & 900219.88536 & 300151.57604 & 3690440.3 & 559077.1 \\
\hline FLAG & 785570.8 & 3325902.8 & 900219.88585 & 300151.18320 & 3690440.7 & 559037.5 \\
\hline FLAG & 785577.4 & 3325916.8 & 900219.62624 & 300151.63189 & 3690463.0 & 559083.0 \\
\hline FLAG & 785579.1 & 3325957.1 & 900219.52395 & 300152.93799 & 3690470.5 & 559215.1 \\
\hline FLAG & 785579.7 & 3325960.0 & 900219.49878 & 300153.03158 & 3690472.6 & 559224.5 \\
\hline FLAG & 785580.4 & 3325965.4 & 900219.46746 & 300153.20619 & 3690475.2 & 559242.2 \\
\hline FLAG & 785580.5 & 3325967.3 & 900219.46190 & 300153.26775 & 3690475.6 & 559248.4 \\
\hline
\end{tabular}




\begin{tabular}{|c|c|c|c|c|c|c|}
\hline \multirow[b]{2}{*}{ Comment } & \multicolumn{2}{|c|}{ UTM (Zone 15) } & \multicolumn{2}{|c|}{ Geographic } & \multicolumn{2}{|c|}{ State Plane, LA South } \\
\hline & $\mathrm{X}, \mathrm{m}$ & $\mathrm{Y}, \mathrm{m}$ & Longitude & Latitude & X, U.S. ft & Y, U.S. ft \\
\hline FLAG & 785583.2 & 3325920.1 & 900219.40679 & 300151.73408 & 3690482.2 & 559093.6 \\
\hline FLAG & 785583.8 & 3325945.0 & 900219.36038 & 300152.54146 & 3690485.4 & 559175.2 \\
\hline FLAG & 785585.3 & 3325988.9 & 900219.26207 & 300153.96453 & 3690492.4 & 559319.0 \\
\hline FLAG & 785585.6 & 3325945.8 & 900219.29249 & 300152.56590 & 3690491.3 & 559177.7 \\
\hline FLAG & 785585.8 & 3325991.1 & 900219.24130 & 300154.03549 & 3690494.1 & 559326.2 \\
\hline FLAG & 785586.0 & 3325992.8 & 900219.23220 & 300154.09048 & 3690494.9 & 559331.8 \\
\hline FLAG & 785586.8 & 3325998.8 & 900219.19658 & 300154.28447 & 3690497.8 & 559351.4 \\
\hline FLAG & 785587.0 & 3325946.1 & 900219.24000 & 300152.57446 & 3690495.9 & 559178.6 \\
\hline FLAG & 785587.1 & 3326002.0 & 900219.18230 & 300154.38805 & 3690498.9 & 559361.9 \\
\hline FLAG & 785587.1 & 3326004.5 & 900219.17989 & 300154.46916 & 3690499.0 & 559370.1 \\
\hline FLAG & 785588.7 & 3325945.8 & 900219.17690 & 300152.56330 & 3690501.5 & 559177.6 \\
\hline FLAG & 785594.0 & 3325986.5 & 900218.93998 & 300153.87935 & 3690520.8 & 559310.7 \\
\hline FLAG & 785597.4 & 3325985.1 & 900218.81455 & 300153.83107 & 3690531.9 & 559306.0 \\
\hline FLAG 3 FT & 785537.7 & 3325930.7 & 900221.09314 & 300152.11622 & 3690333.6 & 559130.5 \\
\hline FLAG 6.5FT & 785543.0 & 3325925.8 & 900220.90025 & 300151.95279 & 3690350.7 & 559114.2 \\
\hline FLAG 7FT & 785546.0 & 3325923.5 & 900220.79060 & 300151.87564 & 3690360.4 & 559106.5 \\
\hline GAS FLAG & 782619.0 & 3325974.9 & 900409.88399 & 300155.98912 & 3680767.3 & 559415.0 \\
\hline GAS LINE & 785292.3 & 3326219.0 & 900229.96547 & 300201.67612 & 3689542.9 & 560087.4 \\
\hline GAS REMOVED & 784857.0 & 3326256.8 & 900246.16086 & 300203.26758 & 3688117.6 & 560232.1 \\
\hline GAS SIGN & 782 & 332 & 90 & 251 & 60.6 & 550 \\
\hline GAS VALVE & 785323.7 & 3326215.4 & 900228.79808 & 300201.53296 & 3689645.6 & 560074.1 \\
\hline GAS VAULT & 784220.9 & 3326188.6 & 900309.94599 & 300201.58724 & 3686029.1 & 560038.9 \\
\hline GATE L10 EA & 785352.4 & 3326110.5 & 900227.82909 & 300158.10540 & 3689734.7 & 559728.8 \\
\hline GATE L10 WE & 785350.4 & 3326119.5 & 900227.89499 & 300158.39908 & 3689728.6 & 559758.4 \\
\hline GATE L11 & 785560.8 & 3325982.7 & 900220.18160 & 300153.78395 & 3690411.8 & 559299.9 \\
\hline GATE L11 & 785570.7 & 3325981.2 & 900219.81390 & 300153.72697 & 3690444.2 & 559294.5 \\
\hline GRATE & 783023.4 & 3325981.1 & 900354.79843 & 300155.85386 & 3682093.4 & 559416.0 \\
\hline GRATE & 783079.6 & 3325985.7 & 900352.69840 & 300155.95632 & 3682277.8 & 559428.4 \\
\hline GRATE & 783093.4 & 3326003.4 & 900352.16687 & 300156.51912 & 3682323.9 & 559485.7 \\
\hline GRATE & 783121.4 & 3326004.8 & 900351.12144 & 300156.54123 & 3682415.8 & 559489.0 \\
\hline GRATE & 783140.9 & 3326004.1 & 900350.39498 & 300156.50227 & 3682479.7 & 559485.7 \\
\hline GRATE & 783180.6 & 3326033.8 & 900348.88618 & 300157.43284 & 3682611.3 & 559581.2 \\
\hline GRATE & 783182.6 & 3326002.2 & 900348.84185 & 300156.40589 & 3682616.3 & 559477.5 \\
\hline GRATE & 783201.3 & 3326002.6 & 900348.14417 & 300156.40330 & 3682677.6 & 559477.9 \\
\hline GRATE & 783219.6 & 3326003.9 & 900347.46054 & 300156.43023 & 3682737.7 & 559481.3 \\
\hline GRATE & 784040.7 & 3326143.0 & 900316.70926 & 300200.25835 & 3685436.1 & 559898.1 \\
\hline GRATE & 785258.3 & 3326245.5 & 900231.20773 & 300202.56444 & 3689432.7 & 560175.9 \\
\hline GRATE & 785269.6 & 3326178.2 & 900230.85127 & 300200.37141 & 3689466.5 & 559954.7 \\
\hline GRATE & 785468.7 & 3325939.6 & 900223.65739 & 300152.46292 & 3690107.8 & 559163.0 \\
\hline GRATE & 785589.0 & 3325986.4 & 900219.12652 & 300153.88031 & 3690504.4 & 559310.6 \\
\hline
\end{tabular}




\begin{tabular}{|c|c|c|c|c|c|c|}
\hline \multirow[b]{2}{*}{ Comment } & \multicolumn{2}{|c|}{ UTM (Zone 15) } & \multicolumn{2}{|c|}{ Geographic } & \multicolumn{2}{|c|}{ State Plane, LA South } \\
\hline & $\mathrm{X}, \mathrm{m}$ & $\mathrm{Y}, \mathrm{m}$ & Longitude & Latitude & X, U.S. ft & Y, U.S. ft \\
\hline GUARD RAIL & 785298.7 & 3326242.0 & 900229.70464 & 300202.41698 & 3689564.9 & 560162.4 \\
\hline GUARD RAIL & 785303.9 & 3326185.2 & 900229.56553 & 300200.56974 & 3689579.3 & 559976.0 \\
\hline GUIDE LINE & 785507.2 & 3325891.4 & 900222.26834 & 300150.86675 & 3690231.7 & 559003.1 \\
\hline HYDRANT & 782371.4 & 3325895.2 & 900419.19284 & 300153.60893 & 3679951.8 & 559165.5 \\
\hline HYDRANT & 782523.7 & 3325956.3 & 900413.45540 & 300155.46484 & 3680454.0 & 559358.6 \\
\hline HYDRANT & 782892.9 & 3325996.8 & 900359.64961 & 300156.47188 & 3681666.3 & 559473.7 \\
\hline HYDRANT & 783282.6 & 3326005.9 & 900345.10943 & 300156.44263 & 3682944.3 & 559484.9 \\
\hline HYDRANT & 784218.1 & 3326193.8 & 900310.04540 & 300201.75830 & 3686020.1 & 560056.1 \\
\hline HYDRANT & 785032.6 & 3326207.7 & 900239.66024 & 300201.52733 & 3688691.0 & 560062.7 \\
\hline HYDRANT & 785121.7 & 3326198.2 & 900236.34697 & 300201.14439 & 3688982.6 & 560027.3 \\
\hline HYDRANT & 785165.4 & 3326201.2 & 900234.71457 & 300201.20507 & 3689126.0 & 560035.1 \\
\hline LAMP & 783687.0 & 3326062.3 & 900329.97578 & 300157.93534 & 3684272.7 & 559650.4 \\
\hline LAMP & 783716.0 & 3326065.2 & 900328.89163 & 300158.00523 & 3684368.0 & 559658.5 \\
\hline LAMP & 783746.3 & 3326068.9 & 900327.75823 & 300158.09999 & 3684467.5 & 559669.2 \\
\hline LAMP & 783752.6 & 3326045.1 & 900327.54614 & 300157.32253 & 3684487.0 & 559590.9 \\
\hline LAMP & 783765.7 & 3326072.6 & 900327.03127 & 300158.20385 & 3684531.2 & 559680.4 \\
\hline LAMP & 783767.6 & 3326099.3 & 900326.93481 & 300159.06855 & 3684538.7 & 559767.9 \\
\hline LAMP & 7837 & 02.1 & 5.34669 & 0159.14630 & 90.4 & 6.3 \\
\hline LAMP & 783 & 332 & 900325.44851 & 300159.99306 & 3684668.3 & 559862.7 \\
\hline LAMP & 783984.6 & 3326196.7 & 900318.74962 & 300202.04753 & 3685254.8 & 560076.8 \\
\hline LAMP & 784028.1 & 3326207.1 & 900317.11755 & 300202.34862 & 3685397.9 & 560108.8 \\
\hline LAMP & 784031.5 & 3326186.4 & 900317.01065 & 300201.67416 & 3685408.1 & 560040.8 \\
\hline LAMP & 784059.1 & 3326206.5 & 900315.96217 & 300202.30325 & 3685499.5 & 560105.4 \\
\hline LAMP & 784207.2 & 3326173.3 & 900310.47155 & 300201.10228 & 3685983.4 & 559989.4 \\
\hline LAMP & 784223.0 & 3326212.2 & 900309.84500 & 300202.35119 & 3686037.1 & 560116.2 \\
\hline LAMP BASE & 783231.4 & 3326021.7 & 900347.00349 & 300156.99793 & 3682777.2 & 559539.1 \\
\hline LAMP BASE & 784078.0 & 3326191.2 & 900315.27210 & 300201.79104 & 3685560.7 & 560054.3 \\
\hline LEVEE CL & 785321.5 & 3326194.7 & 900228.90008 & 300200.86320 & 3689637.4 & 560006.3 \\
\hline LEVEE CL & 785356.6 & 3326103.0 & 900227.67972 & 300157.85853 & 3689748.1 & 559704.0 \\
\hline LEVEE CL & 785615.1 & 3325947.6 & 900218.19077 & 300152.59952 & 3690588.1 & 559182.2 \\
\hline LEVEE STA2 & 785292.3 & 3326219.0 & 900229.96547 & 300201.67612 & 3689542.9 & 560087.4 \\
\hline LIGHT POLE & 782922.1 & 3326007.5 & 900358.55054 & 300156.79475 & 3681762.6 & 559507.3 \\
\hline LIGHT POLE & 782948.2 & 3325976.2 & 900357.60724 & 300155.75748 & 3681846.6 & 559403.5 \\
\hline LIGHT POLE & 782968.7 & 3325942.9 & 900356.87467 & 300154.65997 & 3681912.2 & 559293.3 \\
\hline LIGHT POLE & 785173.4 & 3326291.2 & 900234.32949 & 300204.11841 & 3689156.5 & 560329.7 \\
\hline LIGHT POLE & 785324.1 & 3326177.6 & 900228.81963 & 300200.30621 & 3689645.1 & 559950.1 \\
\hline LIGHT POLE & 785345.3 & 3326095.1 & 900228.10870 & 300157.61170 & 3689710.7 & 559678.6 \\
\hline LIGHT POLE & 785364.4 & 3326052.9 & 900227.43720 & 300156.22649 & 3689771.3 & 559539.4 \\
\hline LIGHT POLE & 785385.9 & 3326014.1 & 900226.67294 & 300154.94958 & 3689839.9 & 559411.2 \\
\hline LIGHT POLE & 785416.2 & 3325979.3 & 900225.57669 & 300153.79506 & 3689937.6 & 559295.6 \\
\hline
\end{tabular}




\begin{tabular}{|c|c|c|c|c|c|c|}
\hline \multirow[b]{2}{*}{ Comment } & \multicolumn{2}{|c|}{ UTM (Zone 15) } & \multicolumn{2}{|c|}{ Geographic } & \multicolumn{2}{|c|}{ State Plane, LA South } \\
\hline & $\mathrm{X}, \mathrm{m}$ & $\mathrm{Y}, \mathrm{m}$ & Longitude & Latitude & X, U.S. ft & Y, U.S. ft \\
\hline LIGHT POLE & 785450.1 & 3325949.1 & 900224.34178 & 300152.78676 & 3690047.3 & 559195.0 \\
\hline LIGHT POLE & 785494.6 & 3325930.6 & 900222.70033 & 300152.14917 & 3690192.3 & 559132.2 \\
\hline LIGHT POLE & 785494.7 & 3325930.5 & 900222.69670 & 300152.14584 & 3690192.6 & 559131.9 \\
\hline LIGHT POLE & 785535.2 & 3325930.4 & 900221.18665 & 300152.10858 & 3690325.4 & 559129.6 \\
\hline LIGHT POLE & 785571.3 & 3325966.2 & 900219.80601 & 300153.23979 & 3690445.4 & 559245.3 \\
\hline LIGHT POLE & 785588.2 & 3325923.0 & 900219.21755 & 300151.82397 & 3690498.7 & 559102.8 \\
\hline LP BASE & 783127.4 & 3326058.8 & 900350.84602 & 300158.28829 & 3682438.0 & 559665.7 \\
\hline LP BASE & 783189.9 & 3326020.8 & 900348.55184 & 300157.00330 & 3682641.1 & 559538.1 \\
\hline MAILBOX & 785179.9 & 3326201.0 & 900234.17407 & 300201.18642 & 3689173.5 & 560033.7 \\
\hline MANHOLE & 782998.2 & 3325936.1 & 900355.78116 & 300154.41479 & 3682008.6 & 559269.6 \\
\hline MANHOLE COV & 785107.0 & 3326274.8 & 900236.82128 & 300203.64201 & 3688938.1 & 560279.1 \\
\hline MANHOLE GRE & 785400.2 & 3326089.3 & 900226.06717 & 300157.37744 & 3689890.4 & 559657.0 \\
\hline $\mathrm{MH}$ & 782537.3 & 3325949.0 & 900412.95524 & 300155.21669 & 3680498.3 & 559334.0 \\
\hline $\mathrm{MH}$ & 784220.1 & 3326180.3 & 900309.98379 & 300201.31861 & 3686026.0 & 560011.8 \\
\hline MH DRAIN & 783780.7 & 3326100.3 & 900326.44537 & 300159.09007 & 3684581.7 & 559770.5 \\
\hline MH DRAIN & 784234.8 & 3326207.2 & 900309.40980 & 300202.17910 & 3686075.5 & 560099.2 \\
\hline MH ELECT & 783269.8 & 3326003.9 & 900345.58864 & 300156.38841 & 3682902.2 & 559478.9 \\
\hline MH PHONE & 783271.1 & 3326005.4 & 900345.53873 & 300156.43599 & 3682906.6 & 559483.8 \\
\hline MH SEWER & 783256.0 & 3326005.5 & 900346.10169 & 300156.45182 & 3682857.1 & 559484.8 \\
\hline MH WATER & 782534.1 & 3325932.4 & 900413.09042 & 300154.68075 & 3680487.0 & 559279.7 \\
\hline MH WATER & 784217.7 & 3326190.4 & 900310.06358 & 300201.64832 & 3686018.7 & 560045.0 \\
\hline MH1 & 784027.8 & 3326080.1 & 900317.25069 & 300158.22831 & 3685390.8 & 559692.5 \\
\hline MH2 & 783929.4 & 3326069.2 & 900320.93036 & 300157.95685 & 3685067.7 & 559661.4 \\
\hline NOPSI & 782511.0 & 3325886.1 & 900413.99601 & 300153.19770 & 3680409.0 & 559129.0 \\
\hline PATH & 782996.3 & 3326021.2 & 900355.77059 & 300157.17750 & 3682006.5 & 559548.7 \\
\hline PATH & 783011.3 & 3326032.5 & 900355.20045 & 300157.53164 & 3682056.2 & 559585.0 \\
\hline PATH & \begin{tabular}{|l}
783041.1 \\
\end{tabular} & 3326036.4 & 900354.08550 & 300157.63337 & 3682154.1 & 559596.4 \\
\hline PATH & 783089.0 & 3326056.4 & 900352.28022 & 300158.24240 & 3682312.0 & 559659.7 \\
\hline POINT & 784847.8 & 3326226.0 & 900246.53358 & 300202.27598 & 3688086.0 & 560131.6 \\
\hline POINT & 784857.0 & 3326227.7 & 900246.18888 & 300202.32342 & 3688116.3 & 560136.7 \\
\hline POINT & 784890.4 & 3326230.5 & 900244.94074 & 300202.38628 & 3688225.9 & 560144.3 \\
\hline POINT & 784903.5 & 3326231.3 & 900244.45148 & 300202.40126 & 3688268.9 & 560146.3 \\
\hline POINT & 785039.0 & 3326243.3 & 900239.38728 & 300202.67701 & 3688713.6 & 560179.1 \\
\hline POINT & 785141.9 & 3326216.4 & 900235.57620 & 300201.71795 & 3689049.7 & 560086.0 \\
\hline POINT & 785173.1 & 3326199.9 & 900234.42870 & 300201.15643 & 3689151.2 & 560030.4 \\
\hline POINT & 785188.7 & 3326196.5 & 900233.85027 & 300201.03303 & 3689202.2 & 560018.5 \\
\hline POINT & 785200.3 & 3326194.8 & 900233.41936 & 300200.96814 & 3689240.1 & 560012.4 \\
\hline POINT & 785206.0 & 3326193.5 & 900233.20807 & 300200.92118 & 3689258.7 & 560007.9 \\
\hline POLE ELECTR & 785411.7 & 3326039.6 & 900225.68631 & 300155.75527 & 3689925.7 & 559493.5 \\
\hline POSLINE & 784838.5 & 3326232.3 & 900246.87430 & 300202.48817 & 3688055.8 & 560152.7 \\
\hline
\end{tabular}




\begin{tabular}{|c|c|c|c|c|c|c|}
\hline \multirow[b]{2}{*}{ Comment } & \multicolumn{2}{|c|}{ UTM (Zone 15) } & \multicolumn{2}{|c|}{ Geographic } & \multicolumn{2}{|c|}{ State Plane, LA South } \\
\hline & $X, m$ & $\mathrm{Y}, \mathrm{m}$ & Longitude & Latitude & X, U.S. ft & Y, U.S. ft \\
\hline POSLINE & 784840.4 & 3326226.5 & 900246.80903 & 300202.29840 & 3688061.8 & 560133.6 \\
\hline POSLINE & 784950.9 & 3326244.8 & 900242.67099 & 300202.79954 & 3688424.9 & 560188.3 \\
\hline POSLINE & 784952.0 & 3326237.3 & 900242.63719 & 300202.55528 & 3688428.1 & 560163.6 \\
\hline POSLINE & 784973.5 & 3326245.6 & 900241.82749 & 300202.80655 & 3688499.0 & 560189.8 \\
\hline POSLINE & 784974.3 & 3326239.6 & 900241.80344 & 300202.61121 & 3688501.4 & 560170.1 \\
\hline POSLINE & 785028.4 & 3326237.1 & 900239.78852 & 300202.48474 & 3688678.6 & 560159.3 \\
\hline POSLINE & 785029.4 & 3326229.6 & 900239.75846 & 300202.24056 & 3688681.5 & 560134.7 \\
\hline POSLINE & 785029.9 & 3326247.9 & 900239.72218 & 300202.83389 & 3688684.0 & 560194.6 \\
\hline POSLINE & 785030.3 & 3326252.3 & 900239.70302 & 300202.97631 & 3688685.6 & 560209.1 \\
\hline POSLINE & 785049.0 & 3326245.5 & 900239.01227 & 300202.74001 & 3688746.5 & 560185.9 \\
\hline POSLINE & 785050.4 & 3326251.5 & 900238.95429 & 300202.93350 & 3688751.4 & 560205.5 \\
\hline POSMH & 785170.9 & 3326207.6 & 900234.50331 & 300201.40810 & 3689144.3 & 560055.8 \\
\hline POSVAULT & 785016.8 & 3326241.9 & 900240.21644 & 300202.65020 & 3688640.8 & 560175.6 \\
\hline POWER POLE & 785502.0 & 3325890.0 & 900222.46359 & 300150.82569 & 3690214.6 & 558998.8 \\
\hline PVC W CONCR & 785104.7 & 3326272.7 & 900236.90907 & 300203.57580 & 3688930.4 & 560272.4 \\
\hline RAMP EDGE & 785497.8 & 3325910.3 & 900222.60060 & 300151.48785 & 3690201.8 & 559065.5 \\
\hline RAMP EDGE1 & 785553.2 & 3325901.2 & 900220.54366 & 300151.14607 & 3690383.0 & 559033.0 \\
\hline ROAD EDGE & 785 & 332 & 9 & 915 & 22.7 & 0.8 \\
\hline ROAD EDGE & 785549.1 & 3325892.5 & 900220.70493 & 300150.86725 & 3690369.1 & 559004.7 \\
\hline ROAD EDGE & 785561.5 & 3325982.3 & 900220.15589 & 300153.77038 & 3690414.1 & 559298.5 \\
\hline ROAD EDGE & 785596.7 & 3325920.2 & 900218.90331 & 300151.72599 & 3690526.5 & 559093.3 \\
\hline ROAD EDGE & 785606.9 & 3325928.9 & 900218.51458 & 300151.99969 & 3690560.3 & 559121.3 \\
\hline ROAD EDGE & 785627.2 & 3325949.5 & 900217.73775 & 300152.65100 & 3690627.9 & 559187.8 \\
\hline ROAD SIGN & 784235.4 & 3326196.4 & 900309.39780 & 300201.82819 & 3686077.0 & 560063.8 \\
\hline ROAD SIGN & 785322.1 & 3326182.6 & 900228.88938 & 300200.47011 & 3689638.8 & 559966.6 \\
\hline ROCK PILE E & 785419.4 & 3326034.7 & 900225.40392 & 300155.58983 & 3689950.7 & 559477.1 \\
\hline RUBBLE & 785106.2 & 3326277.3 & 900236.84870 & 300203.72379 & 3688935.6 & 560287.4 \\
\hline SANSEW & 784909.5 & 3326230.2 & 900244.22881 & 300202.36054 & 3688288.5 & 560142.4 \\
\hline SANSEWER & 784933.4 & 3326233.2 & 900243.33472 & 300202.43784 & 3688367.0 & 560151.1 \\
\hline SD CORPS & 784927.7 & 3326249.7 & 900243.53137 & 300202.97797 & 3688349.1 & 560205.4 \\
\hline SEW CLEAN O & 784027.4 & 3326138.7 & 900317.20934 & 300200.12994 & 3685392.3 & 559884.6 \\
\hline SEW CLEAN O & 784054.9 & 3326141.5 & 900316.18120 & 300200.19781 & 3685482.6 & 559892.5 \\
\hline SEWER & 785179.0 & 3326197.7 & 900234.21081 & 300201.08010 & 3689170.4 & 560022.9 \\
\hline SEWER & 785436.4 & 3325960.0 & 900224.84210 & 300153.15192 & 3690002.9 & 559231.4 \\
\hline SEWER & 785524.2 & 3325885.0 & 900221.64063 & 300150.64482 & 3690287.1 & 558981.3 \\
\hline SEWER & 785596.9 & 3325932.5 & 900218.88398 & 300152.12489 & 3690527.7 & 559133.6 \\
\hline SEWER1 & 785468.5 & 3325939.7 & 900223.66476 & 300152.46633 & 3690107.1 & 559163.3 \\
\hline SEWER2 & 785475.7 & 3325939.1 & 900223.39686 & 300152.44082 & 3690130.7 & 559161.0 \\
\hline SEWER3 HIT & 785486.3 & 3325937.7 & 900223.00297 & 300152.38650 & 3690165.4 & 559155.9 \\
\hline SEWER4 NO H & 785528.6 & 3325934.5 & 900221.42879 & 300152.24715 & 3690303.9 & 559143.4 \\
\hline
\end{tabular}




\begin{tabular}{|c|c|c|c|c|c|c|}
\hline \multirow[b]{2}{*}{ Comment } & \multicolumn{2}{|c|}{ UTM (Zone 15) } & \multicolumn{2}{|c|}{ Geographic } & \multicolumn{2}{|c|}{ State Plane, LA South } \\
\hline & $\mathrm{X}, \mathrm{m}$ & $\mathrm{Y}, \mathrm{m}$ & Longitude & Latitude & X, U.S. ft & Y, U.S. ft \\
\hline SIGN & 783471.5 & 3326121.1 & 900337.95518 & 300200.02290 & 3683569.1 & 559853.5 \\
\hline SIGN & 783572.3 & 3326132.9 & 900334.18513 & 300200.32169 & 3683900.1 & 559887.4 \\
\hline SIGN POST & 785329.7 & 3326148.4 & 900228.63898 & 300159.35411 & 3689662.1 & 559854.1 \\
\hline SIGN POST & 785549.0 & 3325939.9 & 900220.66291 & 300152.40522 & 3690371.0 & 559160.1 \\
\hline SIGN POST & 785564.7 & 3325950.1 & 900220.06765 & 300152.72297 & 3690423.0 & 559192.8 \\
\hline SIGN POST & 785575.6 & 3325997.5 & 900219.61546 & 300154.25170 & 3690461.0 & 559347.7 \\
\hline SIGN POST & 785615.4 & 3325977.1 & 900218.15110 & 300153.55639 & 3690590.5 & 559278.9 \\
\hline SILT FENCE & 784696.3 & 3326229.0 & 900252.17996 & 300202.50022 & 3687589.5 & 560148.6 \\
\hline SILT FENCE & 785286.8 & 3326189.3 & 900230.19920 & 300200.71712 & 3689523.4 & 559990.3 \\
\hline SS & 783409.2 & 3326053.5 & 900340.34306 & 300157.88152 & 3683361.6 & 559634.9 \\
\hline SS & 783434.1 & 3326056.4 & 900339.41179 & 300157.95486 & 3683443.4 & 559643.2 \\
\hline SS & 783469.7 & 3326060.8 & 900338.08009 & 300158.06793 & 3683560.3 & 559655.9 \\
\hline SS & 783492.9 & 3326063.8 & 900337.21211 & 300158.14593 & 3683636.5 & 559664.6 \\
\hline SS & 783524.5 & 3326070.5 & 900336.02736 & 300158.33696 & 3683740.4 & 559685.1 \\
\hline SS & 783548.3 & 3326075.8 & 900335.13481 & 300158.48907 & 3683818.7 & 559701.3 \\
\hline SS & 783572.7 & 3326081.9 & 900334.21911 & 300158.66663 & 3683899.0 & 559720.1 \\
\hline SS & 783787.1 & 3326155.1 & 900326.15414 & 300200.86273 & 3684605.3 & 559949.9 \\
\hline SS & 783794.1 & 3326143.7 & 900325.90406 & 300200.48701 & 4627.7 & 559912.2 \\
\hline SS & 783804.1 & 3326126.0 & 900325.54815 & 300159.90438 & 3684659.7 & 559853.6 \\
\hline SS & 783968.1 & 3326194.5 & 900319.36700 & 300201.98993 & 3685200.6 & 560070.4 \\
\hline SS & 783968.9 & 3326179.0 & 900319.35205 & 300201.48636 & 3685202.5 & 560019.5 \\
\hline SS & 784016.7 & 3326200.3 & 900317.54918 & 300202.13752 & 3685360.2 & 560087.1 \\
\hline SS & 784018.5 & 3326185.5 & 900317.49627 & 300201.65582 & 3685365.4 & 560038.5 \\
\hline SS & 784056.9 & 3326190.4 & 900316.05966 & 300201.78272 & 3685491.5 & 560052.7 \\
\hline SS & 784065.9 & 3326207.0 & 900315.70812 & 300202.31379 & 3685521.8 & 560106.7 \\
\hline STA 23+00 & 785231.9 & 3326218.7 & 900232.21800 & 300201.71707 & 3689344.8 & 560089.3 \\
\hline STA 25+00 & 785173.3 & 3326236.5 & 900234.38595 & 300202.34375 & 3689153.6 & 560150.4 \\
\hline STA $27+00$ & 785114.9 & 3326254.4 & 900236.54636 & 300202.97350 & 3688963.0 & 560211.9 \\
\hline STA 29+00 & 785057.9 & 3326272.0 & 900238.65486 & 300203.59234 & 3688777.0 & 560272.3 \\
\hline STA $31+00$ & 784995.6 & 3326270.3 & 900240.97960 & 300203.58942 & 3688572.7 & 560269.7 \\
\hline STA 33+00 & 784935.5 & 3326267.5 & 900243.22337 & 300203.54895 & 3688375.5 & 560263.4 \\
\hline STA $35+00$ & 784872.9 & 3326265.0 & 900245.56007 & 300203.52030 & 3688170.2 & 560258.2 \\
\hline STA $37+00$ & 784814.2 & 3326256.1 & 900247.75750 & 300203.28073 & 3687977.3 & 560231.8 \\
\hline STA 39+00 & 784754.1 & 3326243.7 & 900250.01051 & 300202.92876 & 3687779.7 & 560194.1 \\
\hline STA 41+00 en & 784696.3 & 3326229.0 & 900252.17996 & 300202.50022 & 3687589.5 & 560148.6 \\
\hline STA $65+22$ & 784075.3 & 3326178.1 & 900315.38536 & 300201.36827 & 3685551.2 & 560011.5 \\
\hline STA $66+00$ & 784053.7 & 3326172.7 & 900316.19599 & 300201.21111 & 3685480.2 & 559994.8 \\
\hline STA $68+00$ & 783993.0 & 3326165.9 & 900318.46596 & 300201.04120 & 3685280.9 & 559975.4 \\
\hline STA 69+00 & 783976.5 & 3326148.1 & 900319.09832 & 300200.47745 & 3685225.9 & 559917.9 \\
\hline STA $72+20$ & 783976.6 & 3326057.1 & 900319.18195 & 300157.52484 & 3685221.9 & 559619.5 \\
\hline
\end{tabular}




\begin{tabular}{|c|c|c|c|c|c|c|}
\hline \multirow[b]{2}{*}{ Comment } & \multicolumn{2}{|c|}{ UTM (Zone 15) } & \multicolumn{2}{|c|}{ Geographic } & \multicolumn{2}{|c|}{ State Plane, LA South } \\
\hline & $\mathrm{X}, \mathrm{m}$ & $\mathrm{Y}, \mathrm{m}$ & Longitude & Latitude & X, U.S. ft & Y, U.S. ft \\
\hline STA 75+33 & 783885.4 & 3326028.3 & 900322.61032 & 300156.66658 & 3684921.5 & 559529.5 \\
\hline STA $76+43$ & 783856.3 & 3326035.9 & 900323.68813 & 300156.93746 & 3684826.5 & 559555.8 \\
\hline STA 78+00 & 783821.2 & 3326065.0 & 900324.96904 & 300157.91093 & 3684712.8 & 559652.9 \\
\hline STA $79+00$ & 783794.9 & 3326078.8 & 900325.93649 & 300158.38063 & 3684627.3 & 559699.4 \\
\hline STGRATE & 785048.3 & 3326241.6 & 900239.04214 & 300202.61406 & 3688744.0 & 560173.1 \\
\hline STOP SIGN & 784236.2 & 3326221.9 & 900309.34347 & 300202.65488 & 3686080.8 & 560147.4 \\
\hline STREE SIGN & 784234.2 & 3326214.7 & 900309.42496 & 300202.42294 & 3686073.9 & 560123.9 \\
\hline STREET & 785539.8 & 3325928.6 & 900221.01686 & 300152.04632 & 3690340.3 & 559123.5 \\
\hline STRMGRT & 784957.3 & 3326235.9 & 900242.44091 & 300202.50541 & 3688445.5 & 560158.8 \\
\hline STRMGRTE & 784969.1 & 3326237.1 & 900241.99975 & 300202.53446 & 3688484.2 & 560162.1 \\
\hline SWING SET E & 785148.8 & 3326312.6 & 900235.22616 & 300204.83337 & 3689076.9 & 560401.1 \\
\hline SWING SET E & 785159.3 & 3326310.9 & 900234.83627 & 300204.76940 & 3689111.3 & 560395.0 \\
\hline TABLE & 785122.7 & 3326273.6 & 900236.23700 & 300203.58990 & 3688989.5 & 560274.5 \\
\hline TABLE & 785143.3 & 3326270.6 & 900235.47174 & 300203.47529 & 3689056.9 & 560263.6 \\
\hline TABLE & 785151.9 & 3326299.5 & 900235.12320 & 300204.40574 & 3689086.4 & 560358.0 \\
\hline TABLE & 785158.5 & 3326285.2 & 900234.89088 & 300203.93624 & 3689107.4 & 560310.8 \\
\hline TABLE & 785165.4 & 3326302.3 & 900234.61710 & 300204.48526 & 3689130.8 & 560366.5 \\
\hline TABLE CAN & 785032.6 & 84.3 & 642 & 263 & 94.6 & 560313.8 \\
\hline TILE & 783272.2 & 3326071.5 & 900345.43441 & 300158.57973 & 3682913.3 & 559700.4 \\
\hline TILE & 783273.8 & 3326062.0 & 900345.38385 & 300158.27016 & 3682918.1 & 559669.2 \\
\hline TILE & 783320.0 & 3326077.1 & 900343.64663 & 300158.72159 & 3683070.3 & 559716.5 \\
\hline TILE & 783320.7 & 3326066.9 & 900343.63030 & 300158.39006 & 3683072.1 & 559683.0 \\
\hline TRAFFIC BAR & 785604.6 & 3326002.3 & 900218.52948 & 300154.38308 & 3690556.3 & 559362.0 \\
\hline TRANSFORM & 785088.8 & 3326287.4 & 900237.48779 & 300204.06608 & 3688879.0 & 560321.3 \\
\hline TRASH CAN & 785138.1 & 3326271.7 & 900235.66458 & 300203.51534 & 3689039.9 & 560267.5 \\
\hline TREE & 785238.3 & 3326187.3 & 900232.00963 & 300200.69292 & 3689364.3 & 559986.0 \\
\hline TREE & 785259.6 & 3326160.4 & 900231.24132 & 300159.80228 & 3689432.9 & 559896.8 \\
\hline TREE & 785575.4 & 3325955.7 & 900219.66327 & 300152.89567 & 3690458.3 & 559210.7 \\
\hline TREE & 785576.5 & 3325926.2 & 900219.65073 & 300151.93763 & 3690460.5 & 559113.9 \\
\hline VALVE & 785547.7 & 3325962.3 & 900220.68976 & 300153.13308 & 3690367.9 & 559233.6 \\
\hline VALVE GAS & 784225.6 & 3326185.2 & 900309.77400 & 300201.47300 & 3686044.3 & 560027.6 \\
\hline VALVE WATER & 784224.0 & 3326207.1 & 900309.81262 & 300202.18489 & 3686040.1 & 560099.4 \\
\hline VAULT & 785101.4 & 3326283.1 & 900237.02210 & 300203.91600 & 3688920.1 & 560306.6 \\
\hline WALL & 782963.7 & 3325951.9 & 900357.05250 & 300154.95615 & 3681896.3 & 559323.1 \\
\hline WALL-FENCE & 783009.3 & 3325952.9 & 900355.35118 & 300154.95063 & 3682045.8 & 559324.2 \\
\hline WATER & 784951.8 & 3326252.1 & 900242.63039 & 300203.03563 & 3688428.2 & 560212.1 \\
\hline WATER & 785181.0 & 3326199.4 & 900234.13460 & 300201.13358 & 3689177.1 & 560028.4 \\
\hline WATER COVER & 785500.6 & 3325881.7 & 900222.52380 & 300150.55757 & 3690209.6 & 558971.6 \\
\hline WATER FOUNT & 784267.5 & 3326219.0 & 900308.17910 & 300202.53461 & 3686183.3 & 560136.4 \\
\hline WATER PIPE & 783271.0 & 3326010.2 & 900345.53786 & 300156.59181 & 3682906.5 & 559 \\
\hline
\end{tabular}




\begin{tabular}{|l|l|l|l|l|l|l|}
\hline \multirow{2}{*}{ Comment } & \multicolumn{2}{|c|}{ UTM (Zone 15) } & \multicolumn{2}{c|}{ Geographic } & \multicolumn{1}{c|}{ State Plane, LA South } \\
\cline { 2 - 7 } & $\mathrm{X}, \mathrm{m}$ & $\mathrm{Y}, \mathrm{m}$ & Longitude & Latitude & X, U.S. ft & Y, U.S. $\mathrm{ft}$ \\
\hline WATER VALVE & 785192.6 & 3326268.6 & 900233.63533 & 300203.36904 & 3689218.4 & 560254.7 \\
\hline WATERFOUN & 782995.0 & 3325992.0 & 900355.84700 & 300156.23116 & 3682000.8 & 559453.0 \\
\hline WET AREA & 785164.6 & 3326257.6 & 900234.69002 & 300203.03564 & 3689126.1 & 560220.0 \\
\hline XING & 783982.9 & 3326074.8 & 900318.93004 & 300158.09386 & 3685243.4 & 559677.2 \\
\hline
\end{tabular}

Note: NAD83. 


\section{Appendix B: EM31 Conductivity and In-phase Results, LPV101, LPV102, LPV103, and LPV104}




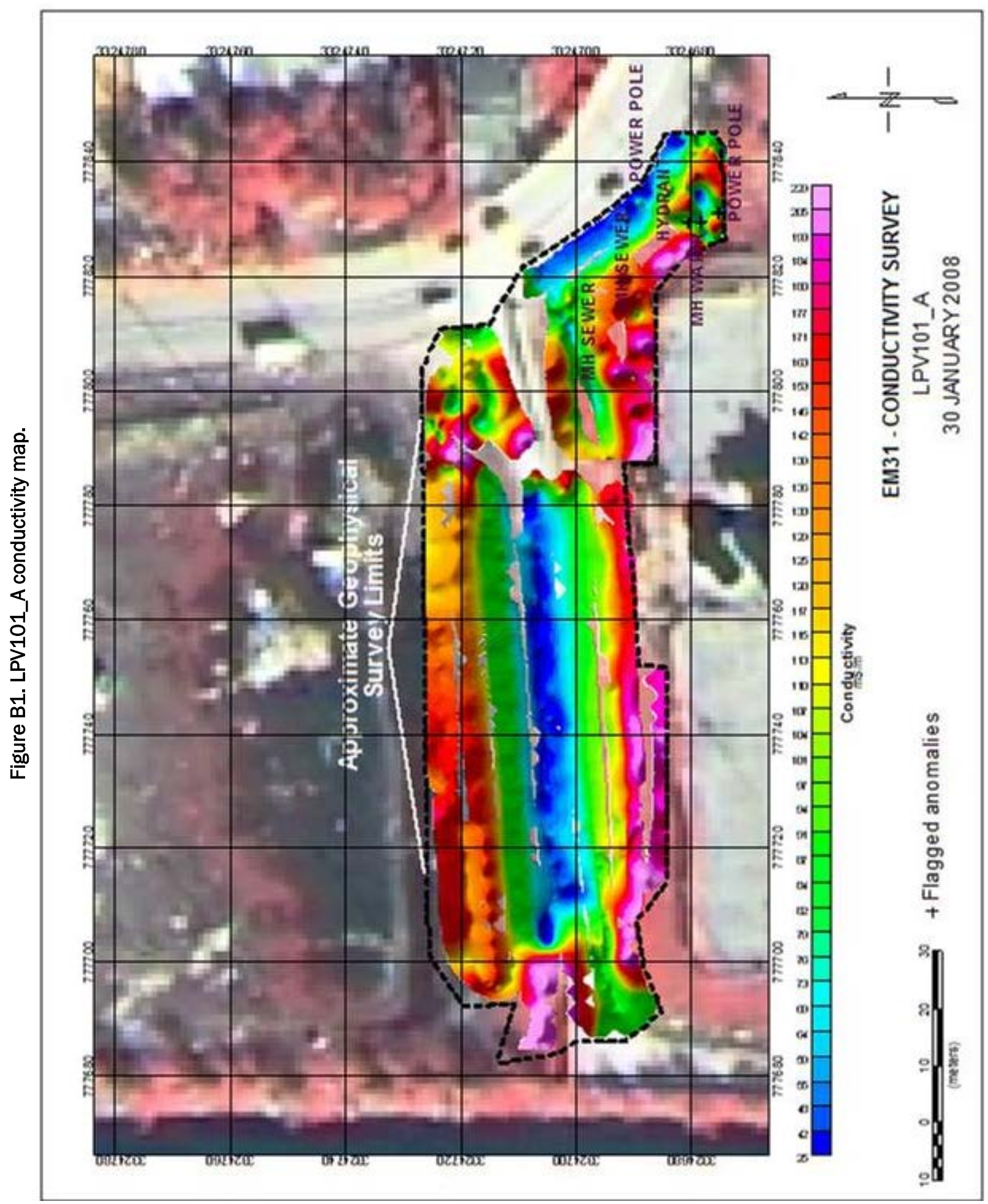




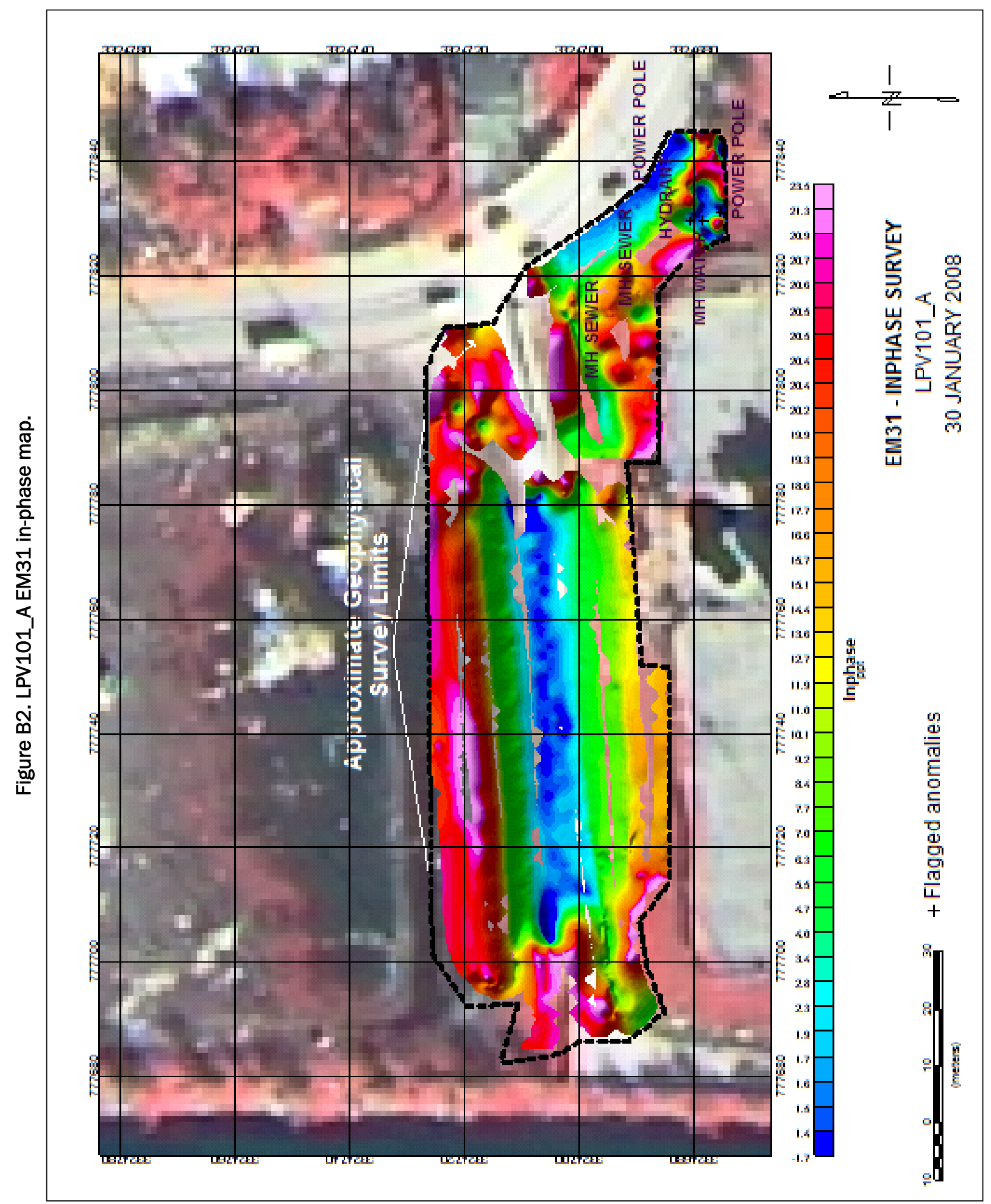


Figure B3. LPV101_B EM31 conductivity map.

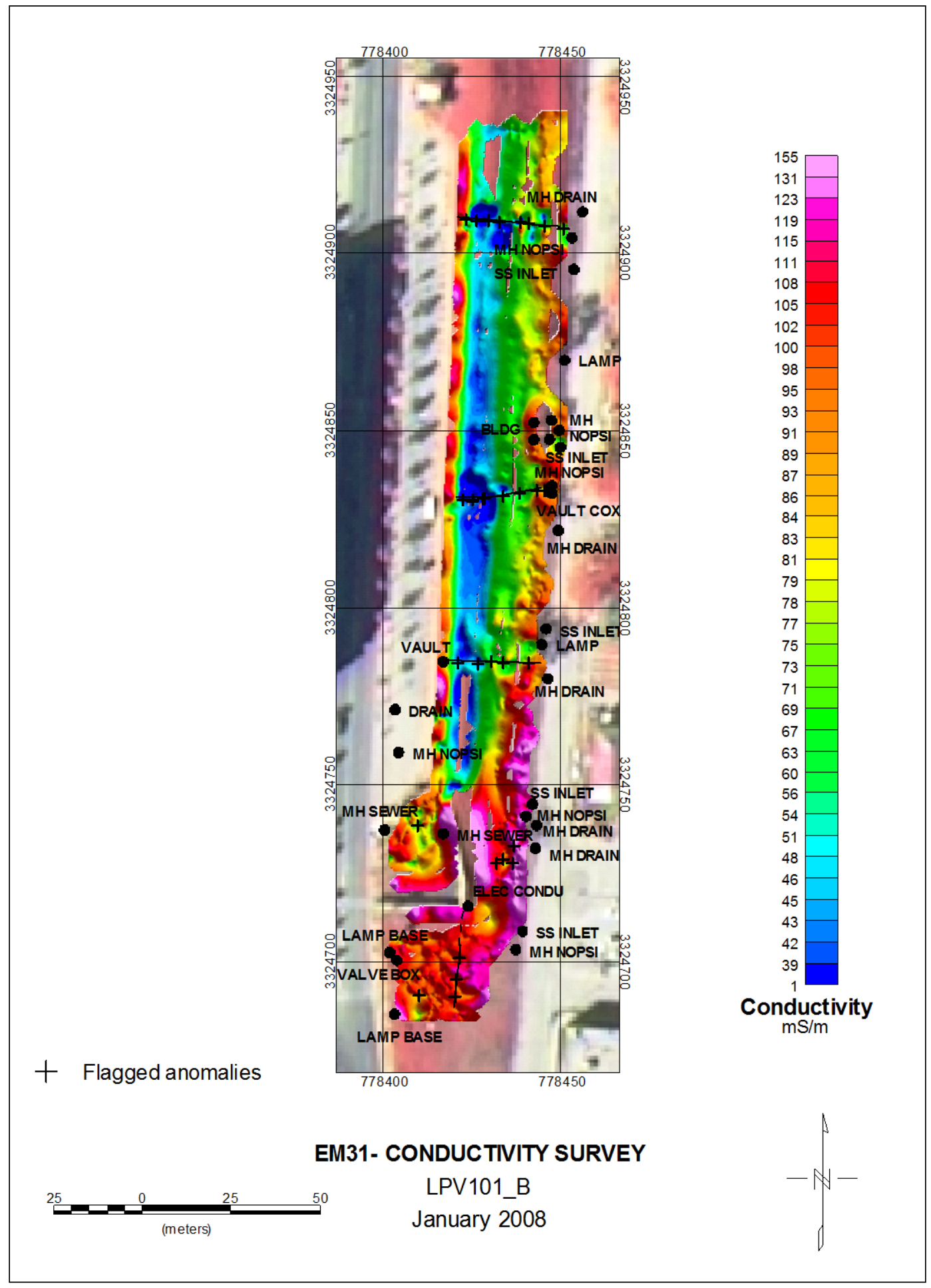


Figure B4. LPV101_B EM31 in-phase map.

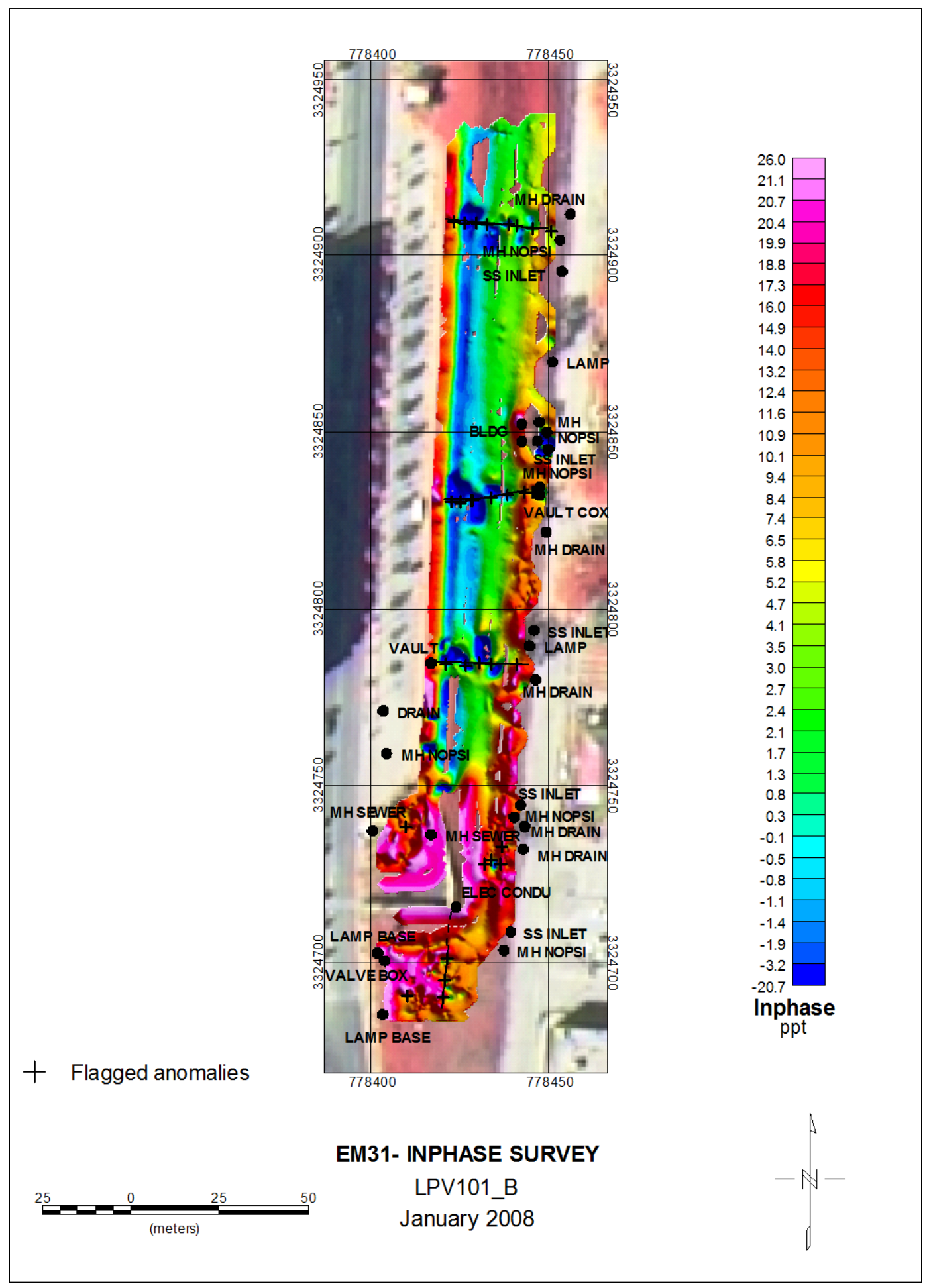


Figure B5. LPV101_C EM31 conductivity map.

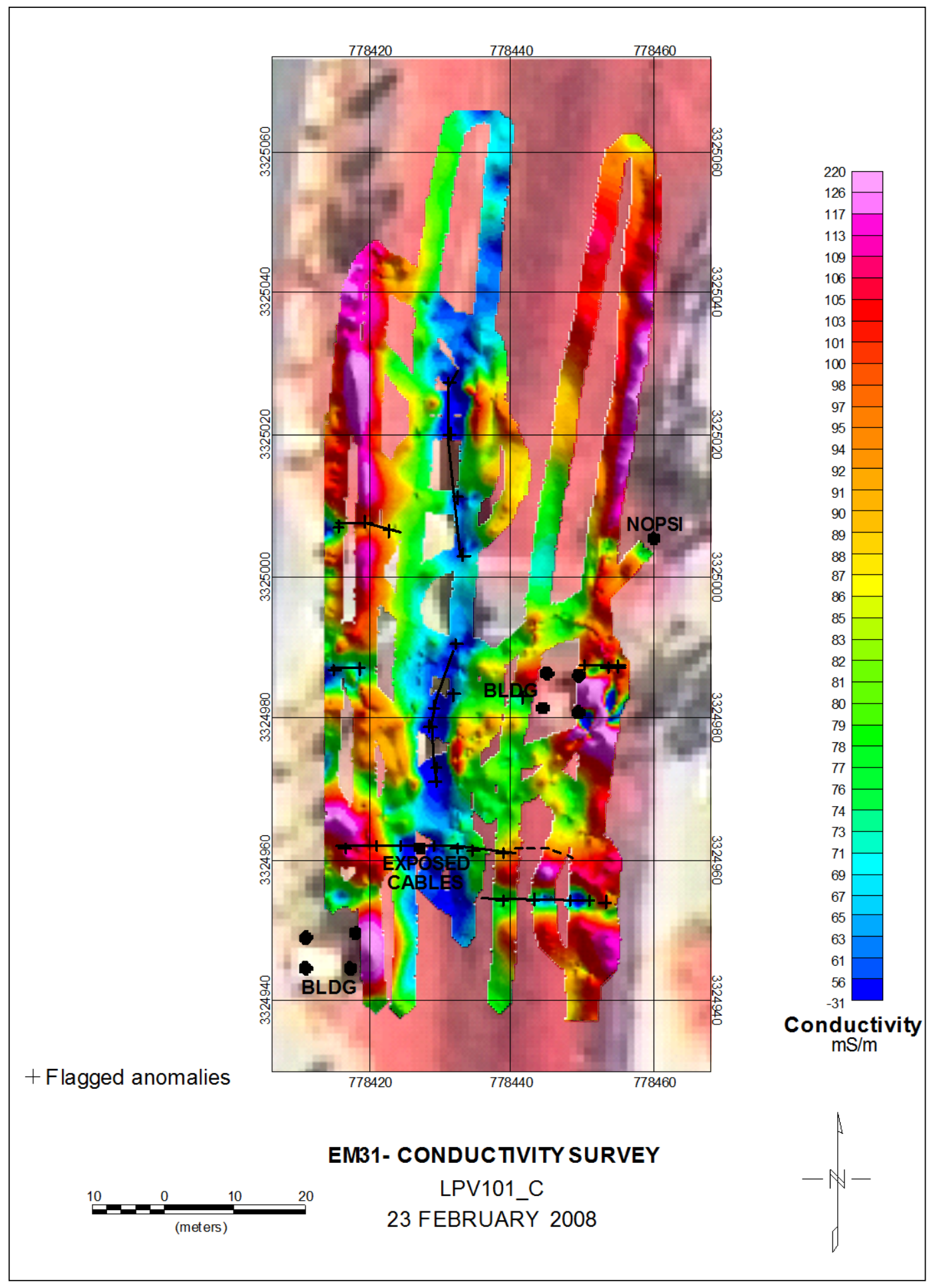


Figure B6. LPV101_C EM31 in-phase map.

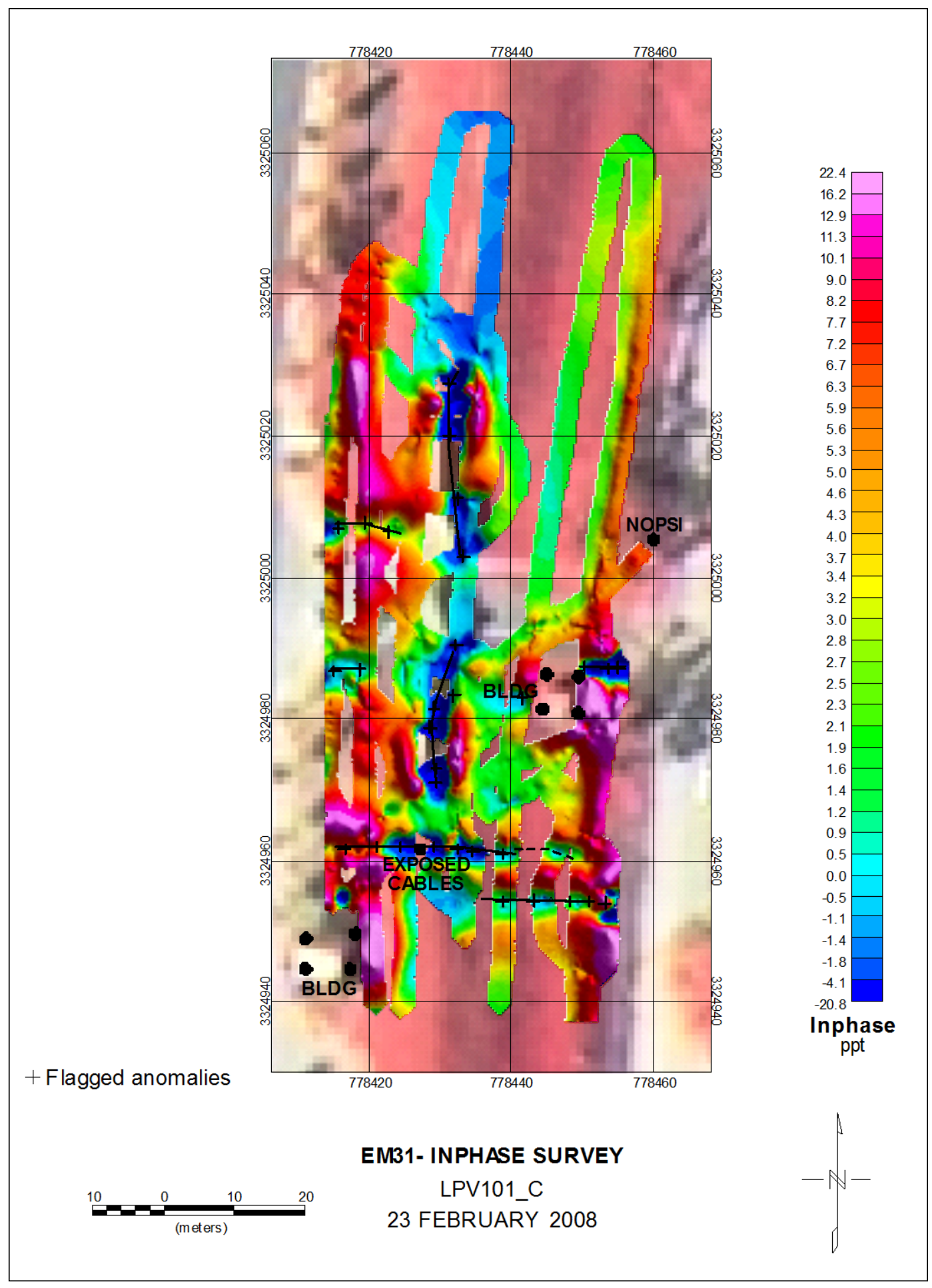




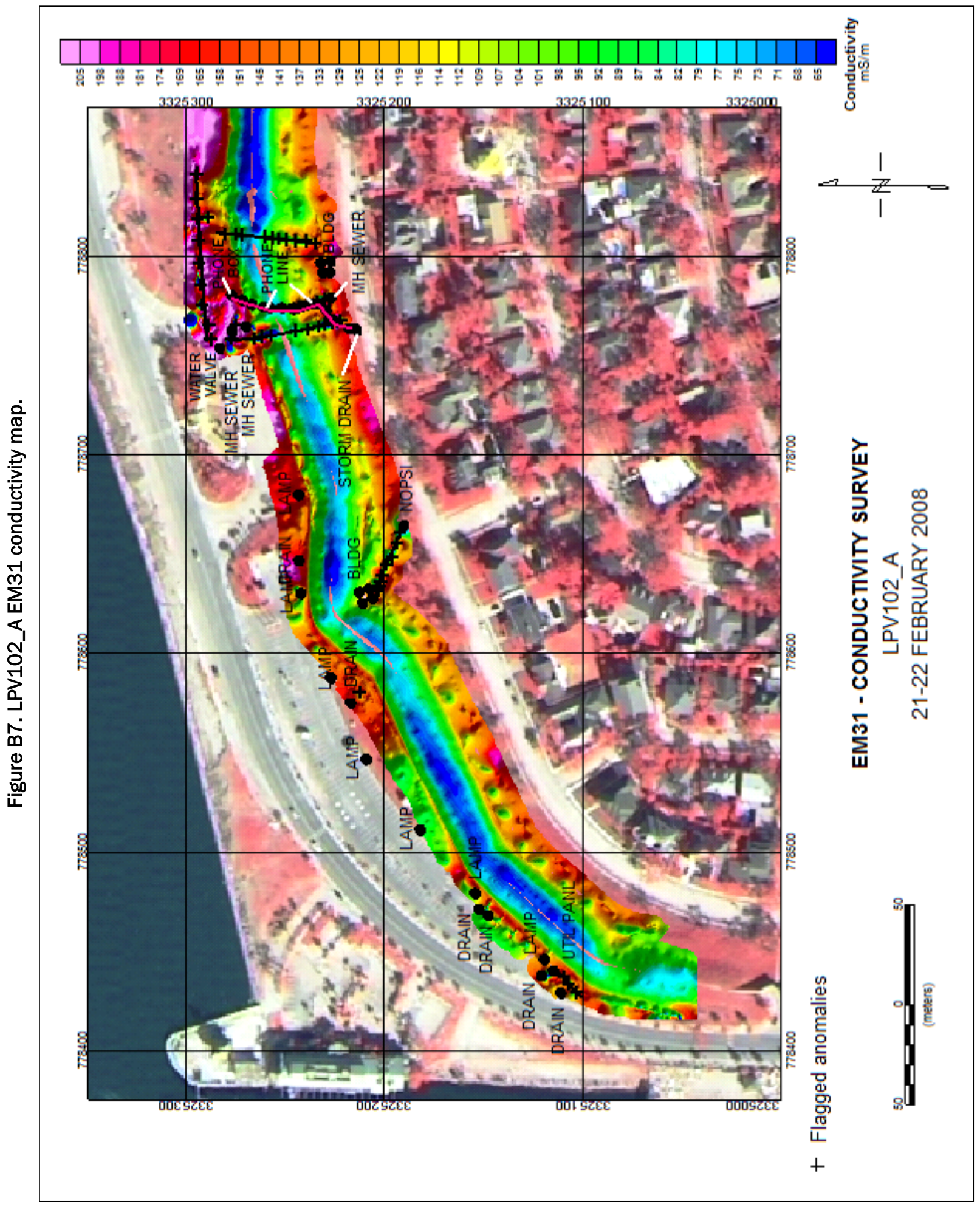




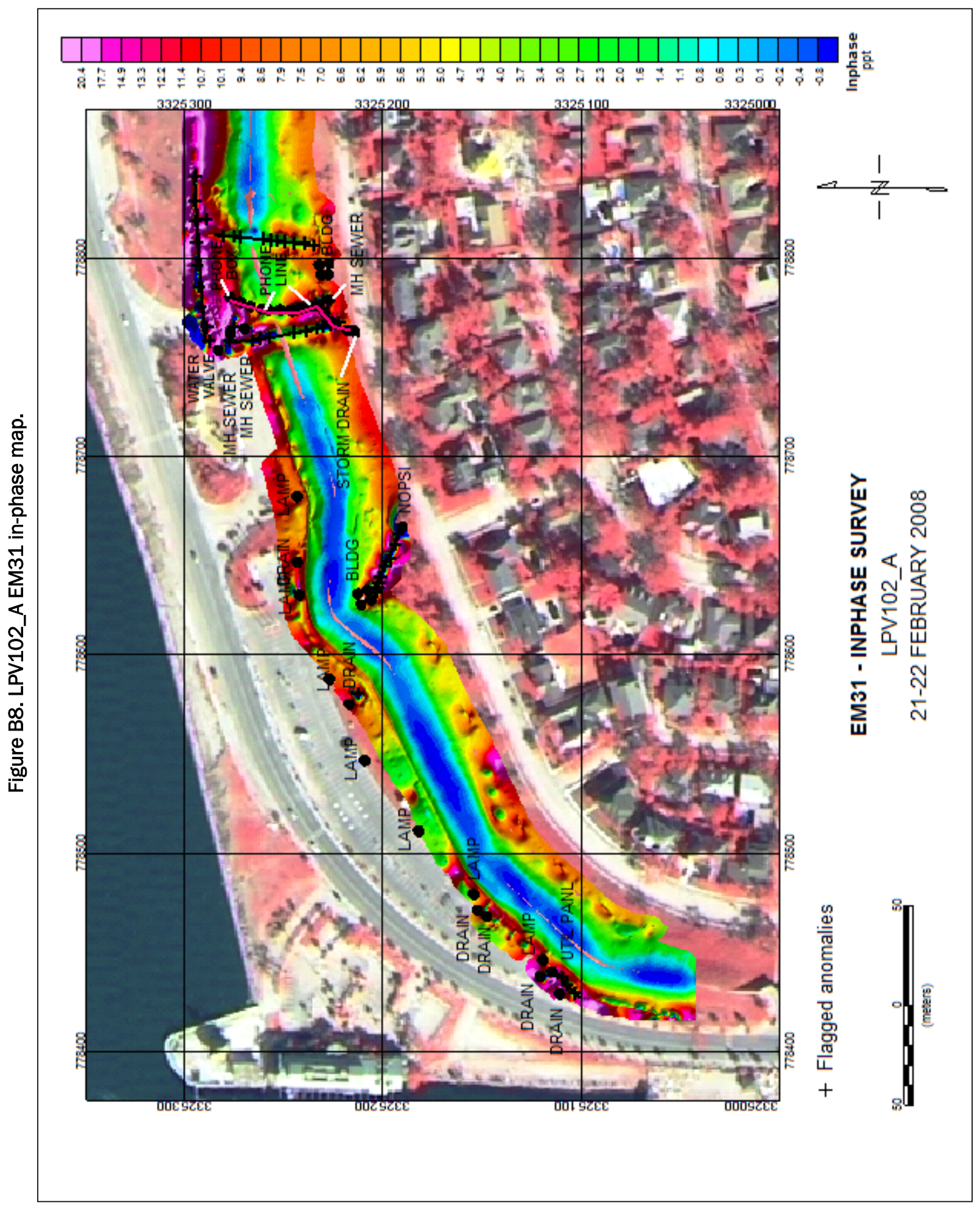




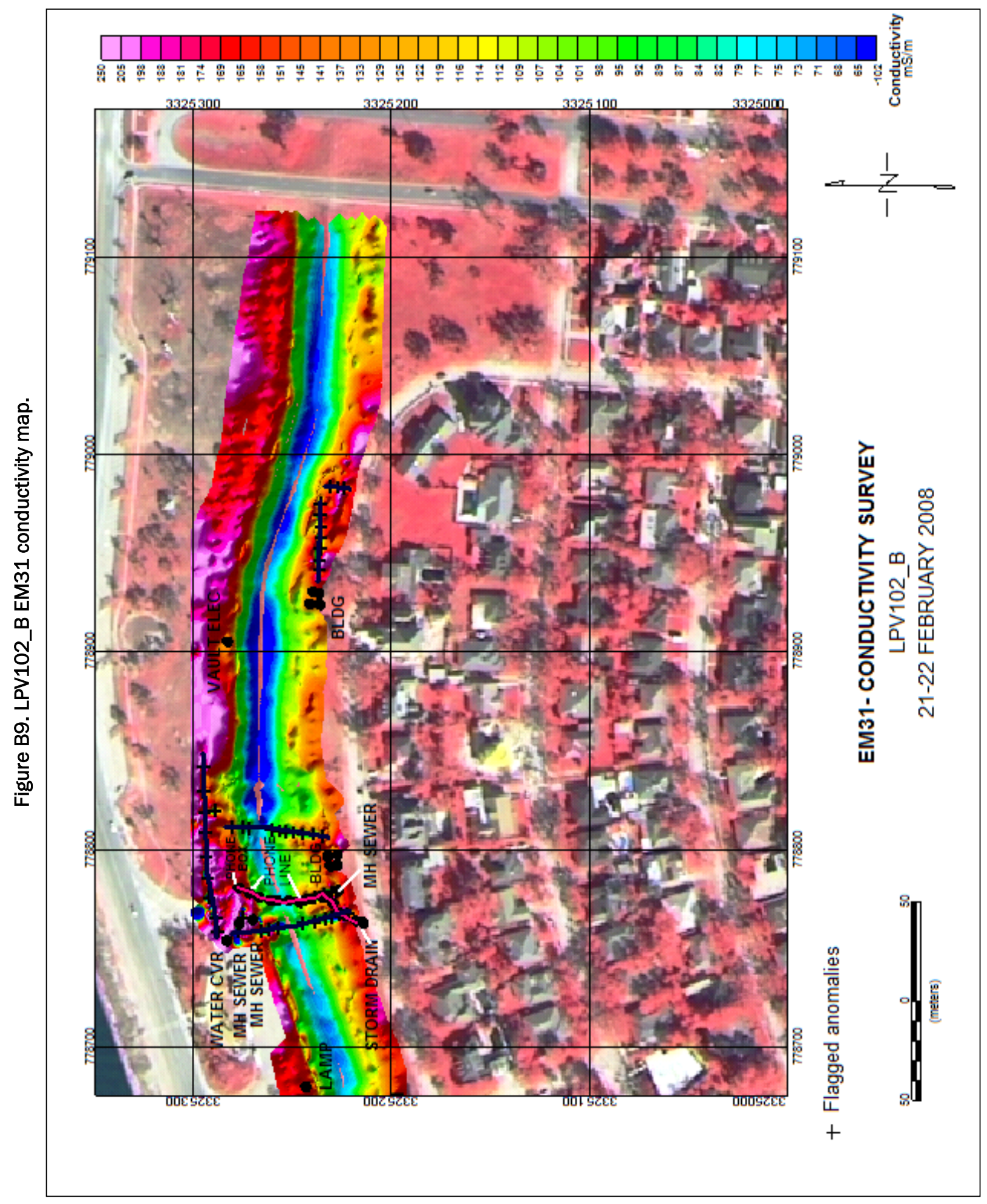




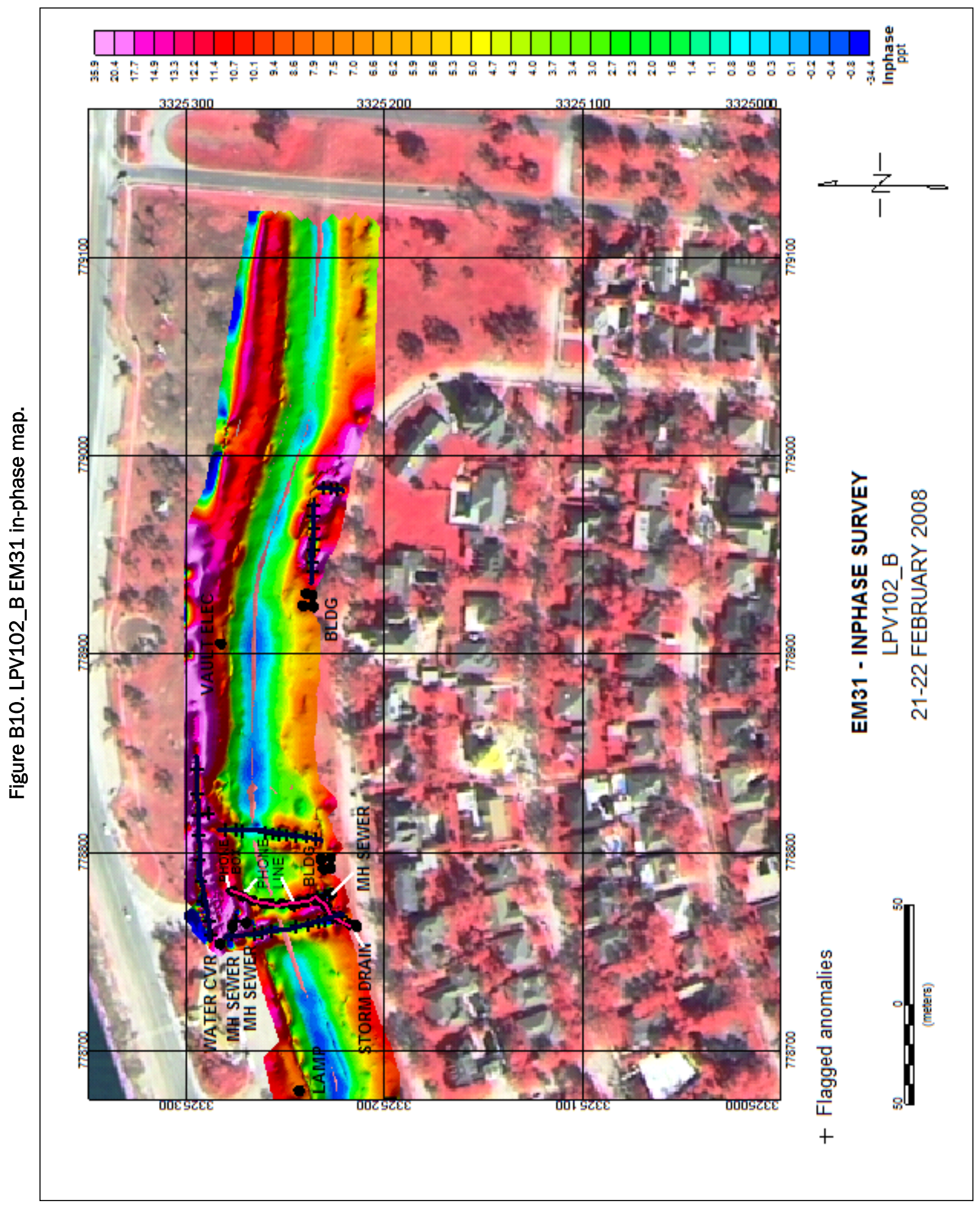




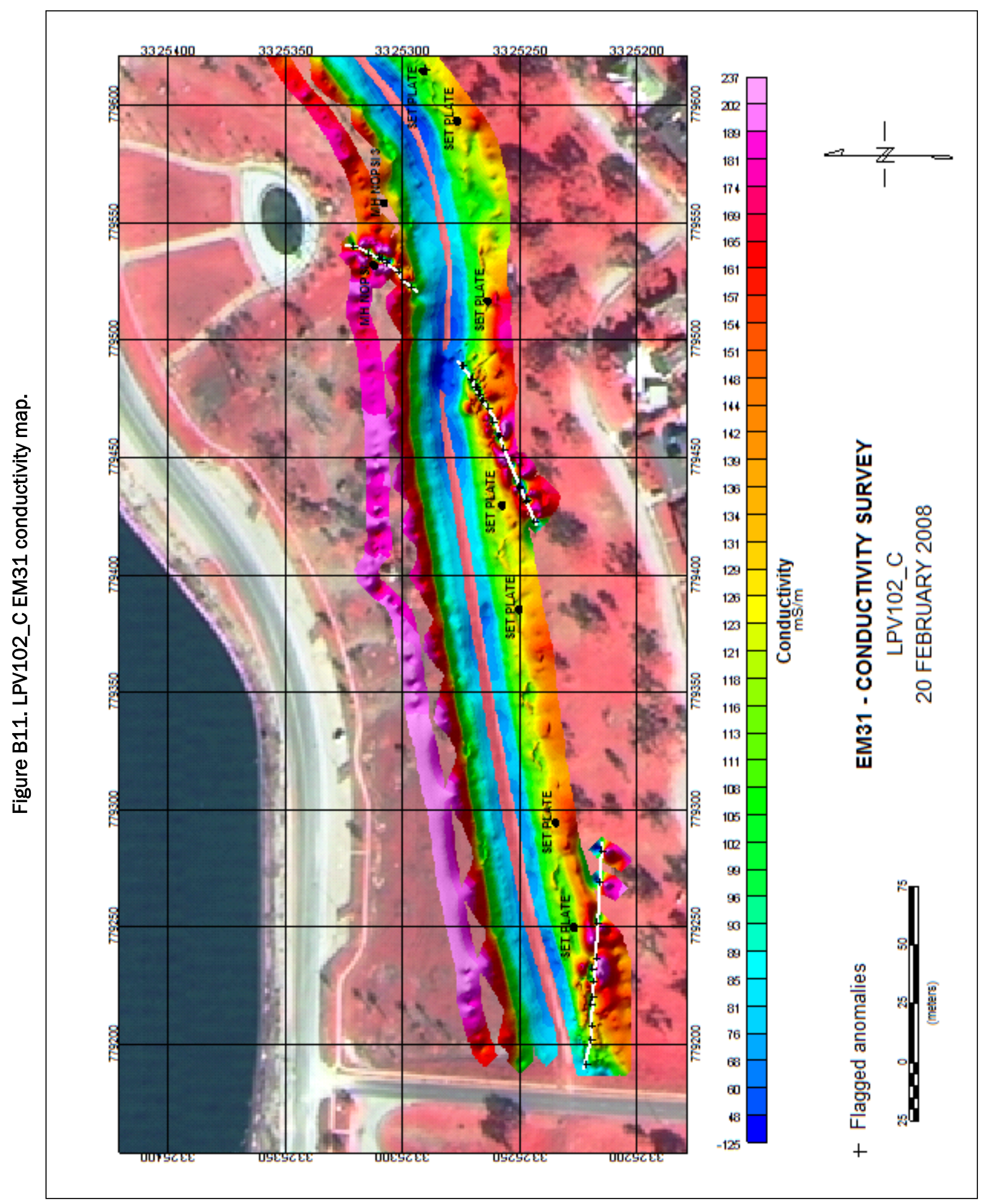




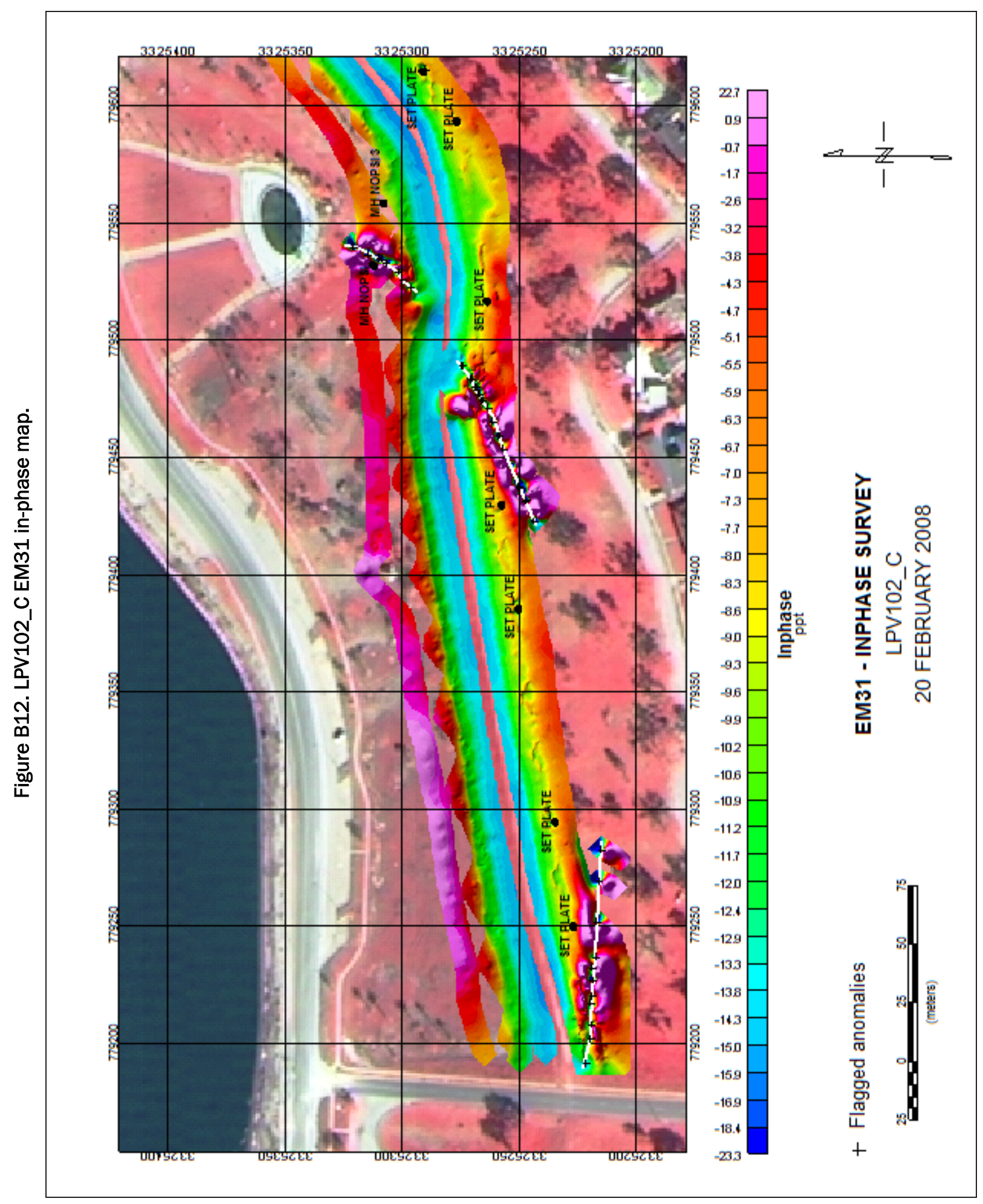




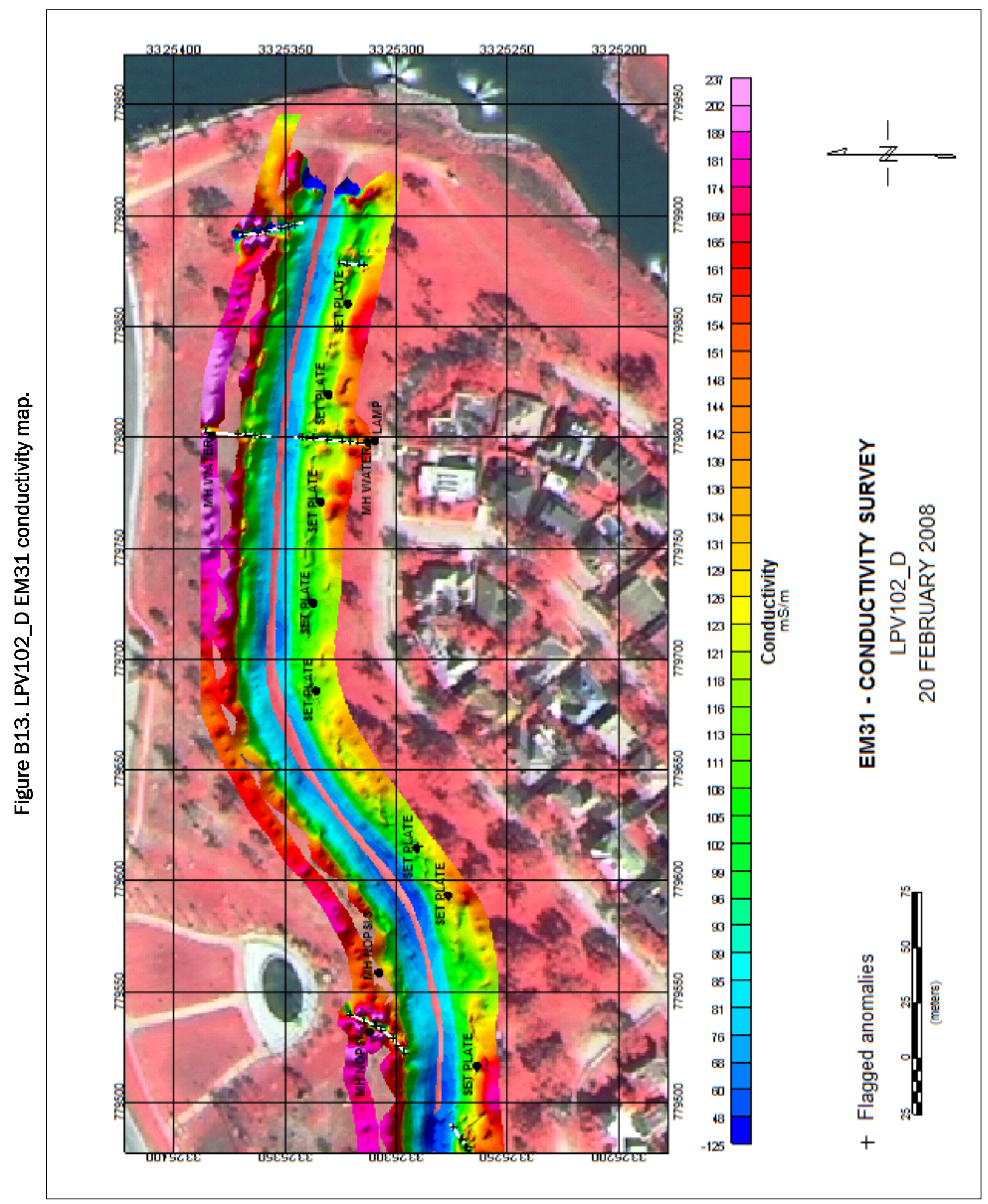




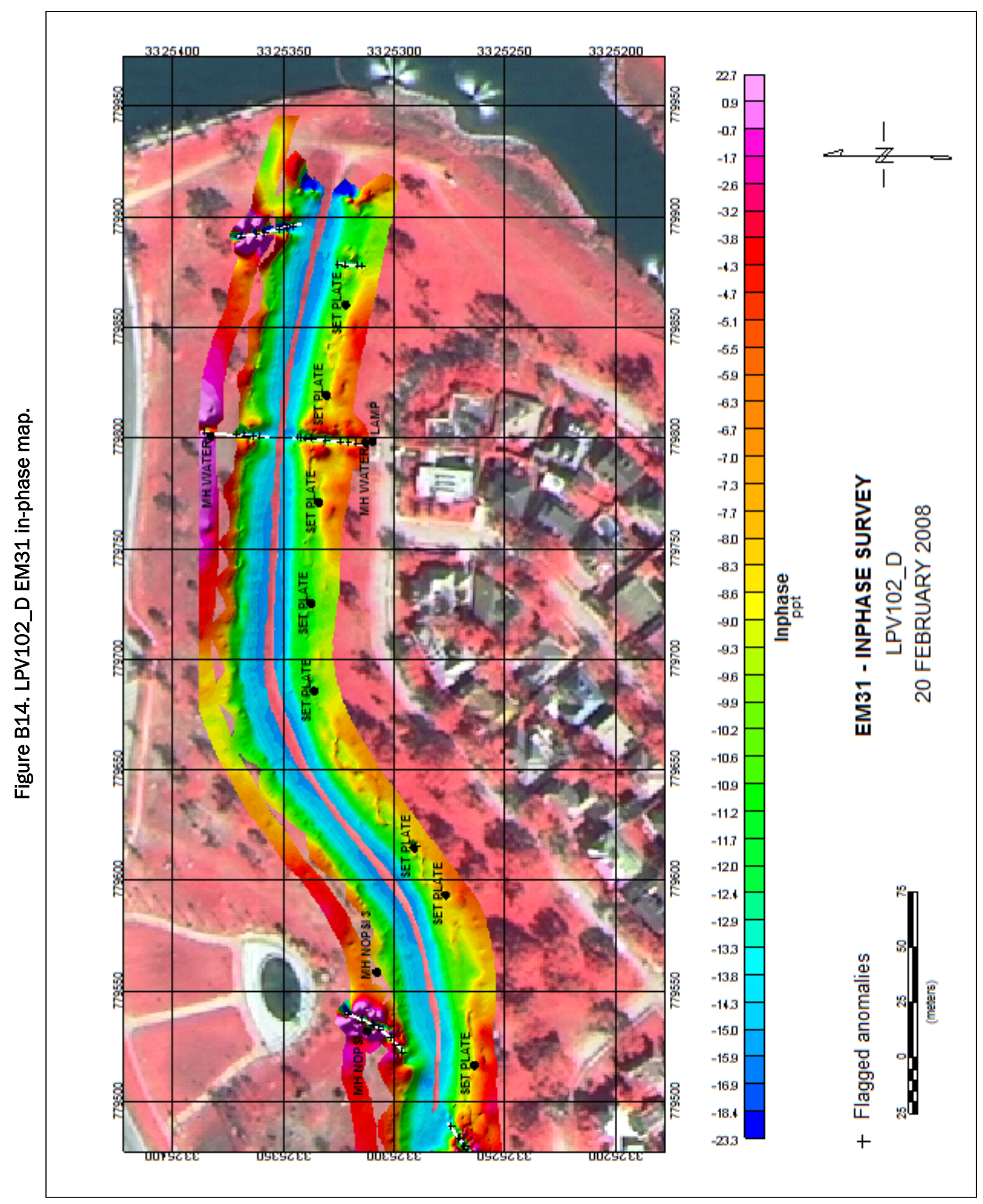


Figure B15. LPV102_E EM31 conductivity map.

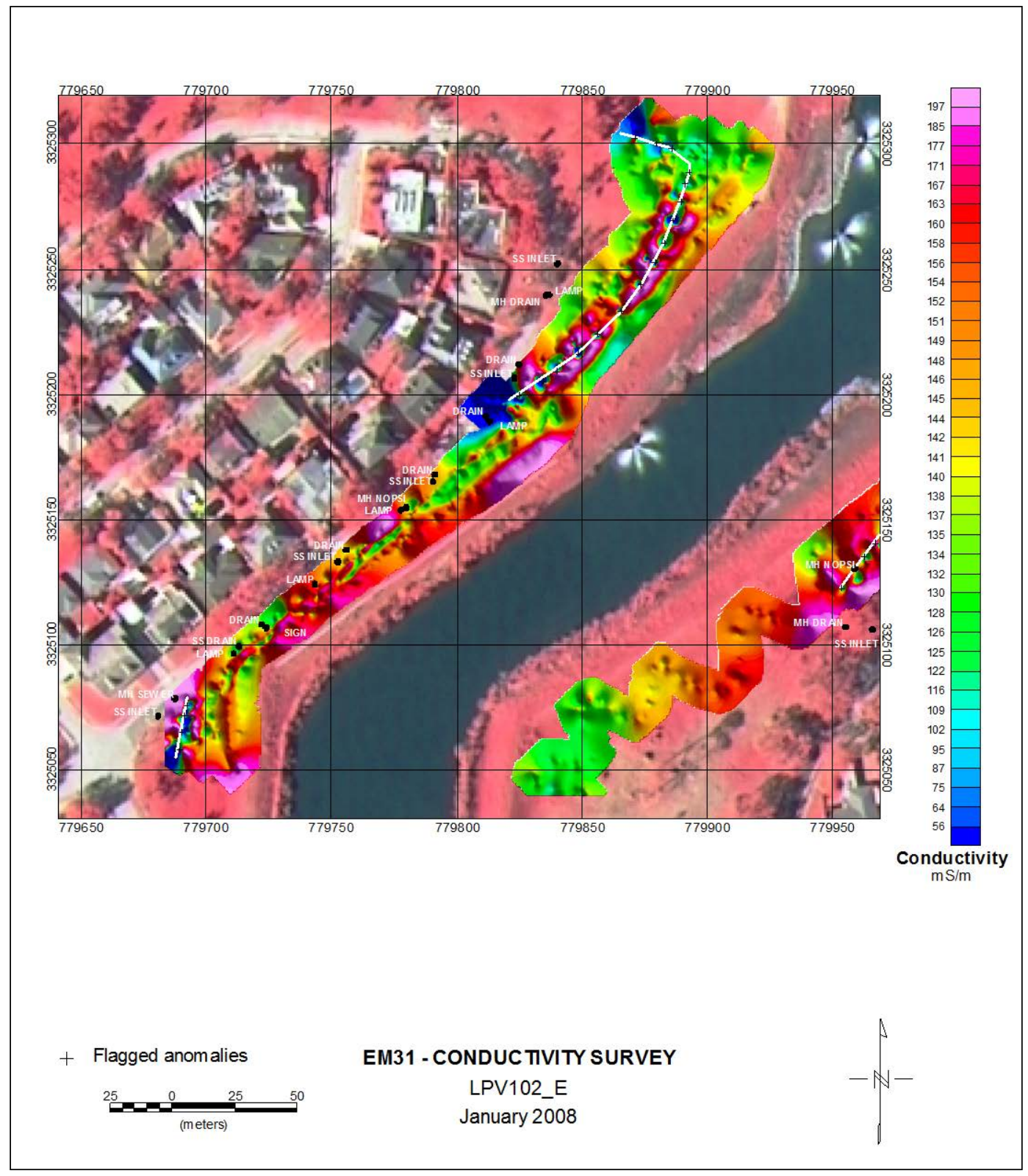


Figure B16. LPV102_E EM31 in-phase map.

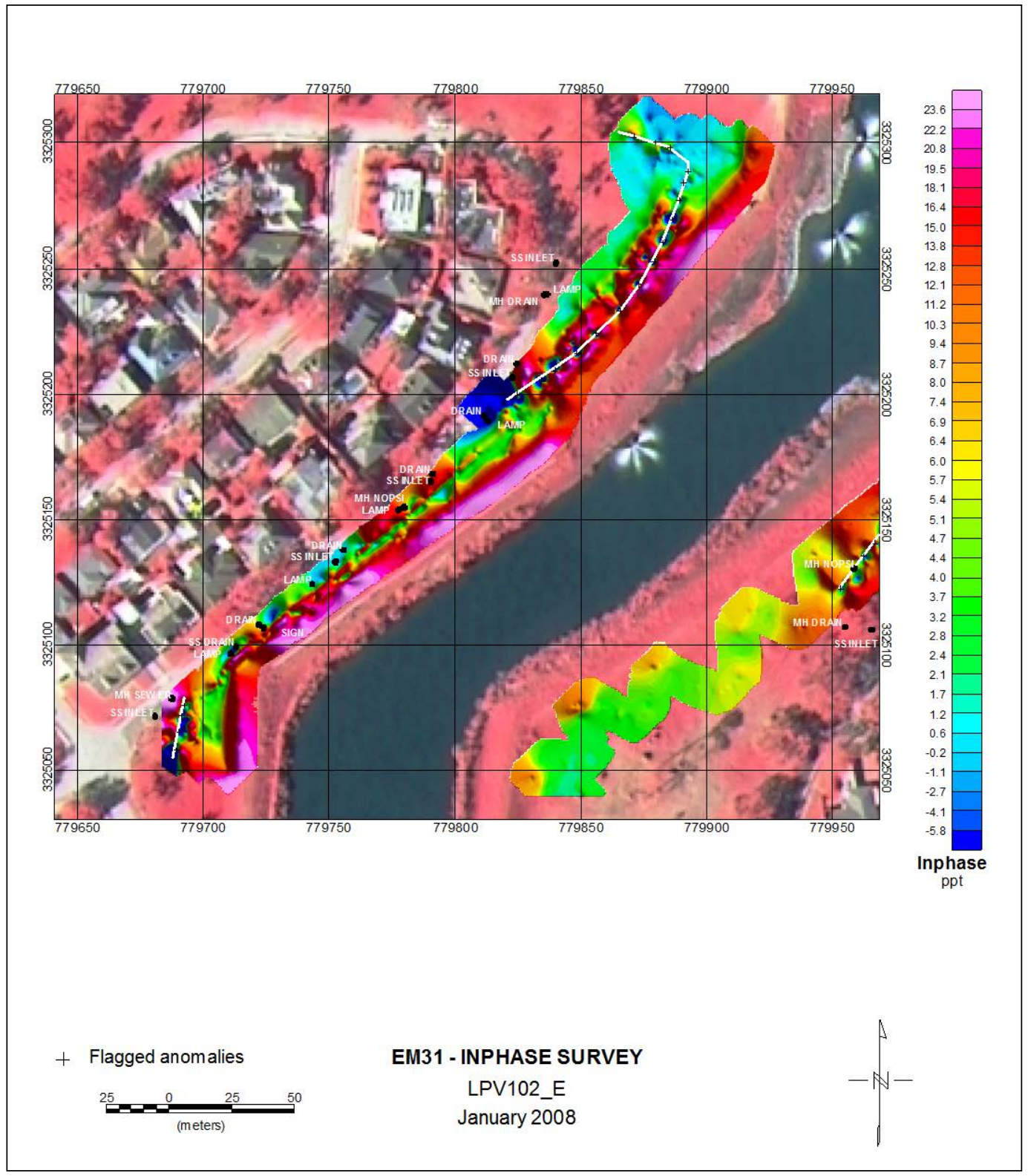


Figure B17. LPV103_AA EM31 conductivity map.

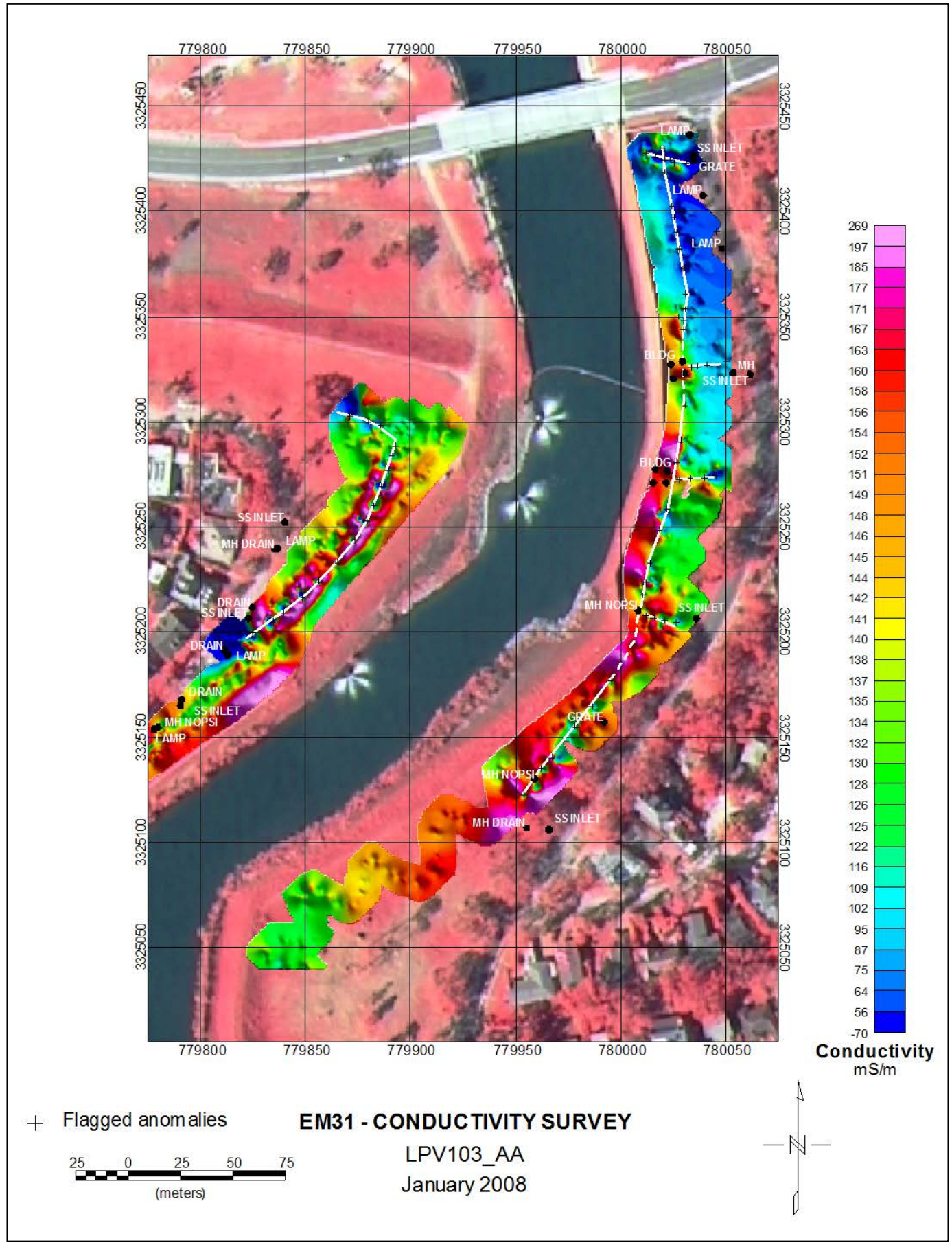


Figure B18. LPV103_AA EM31 in-phase map.

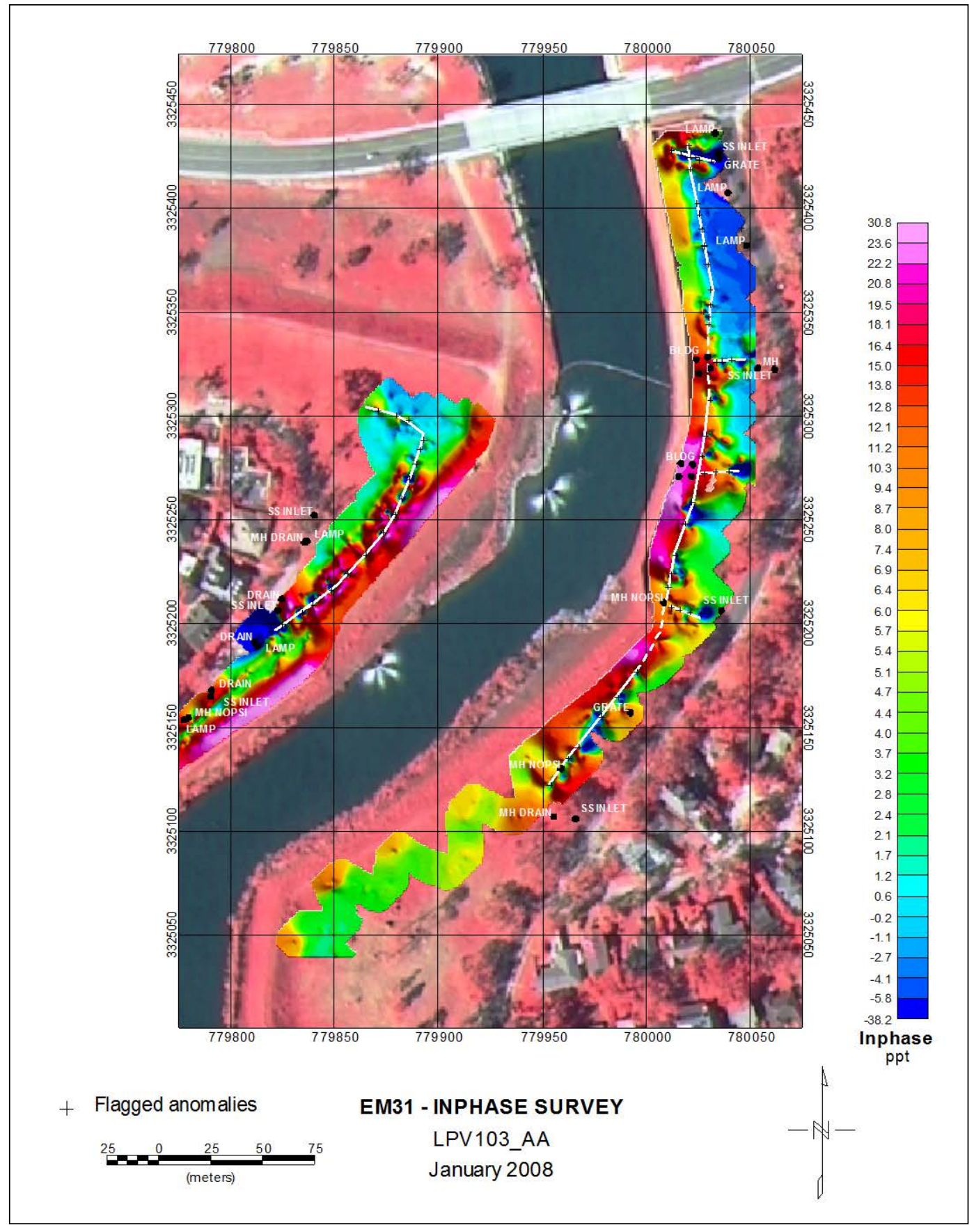




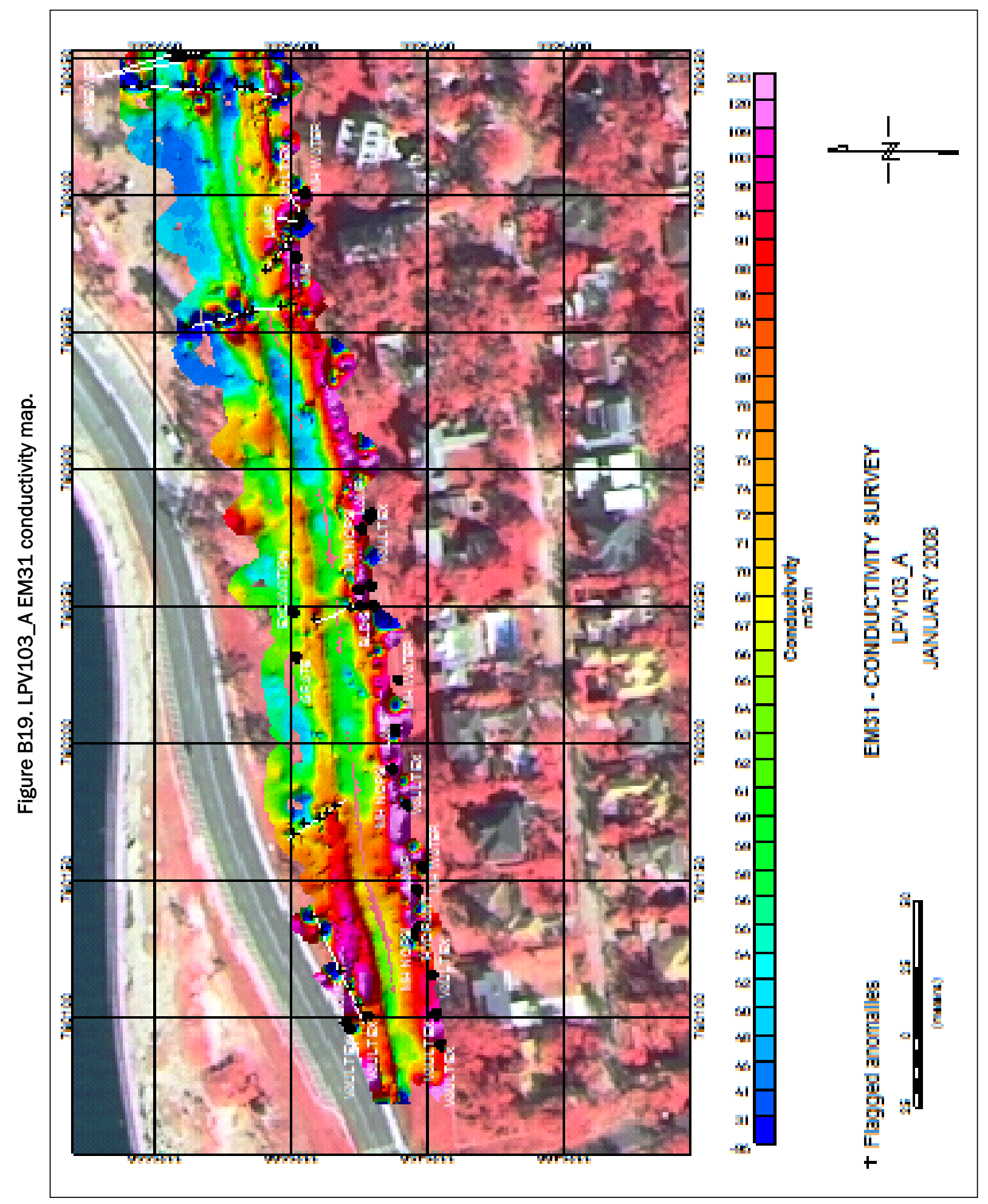




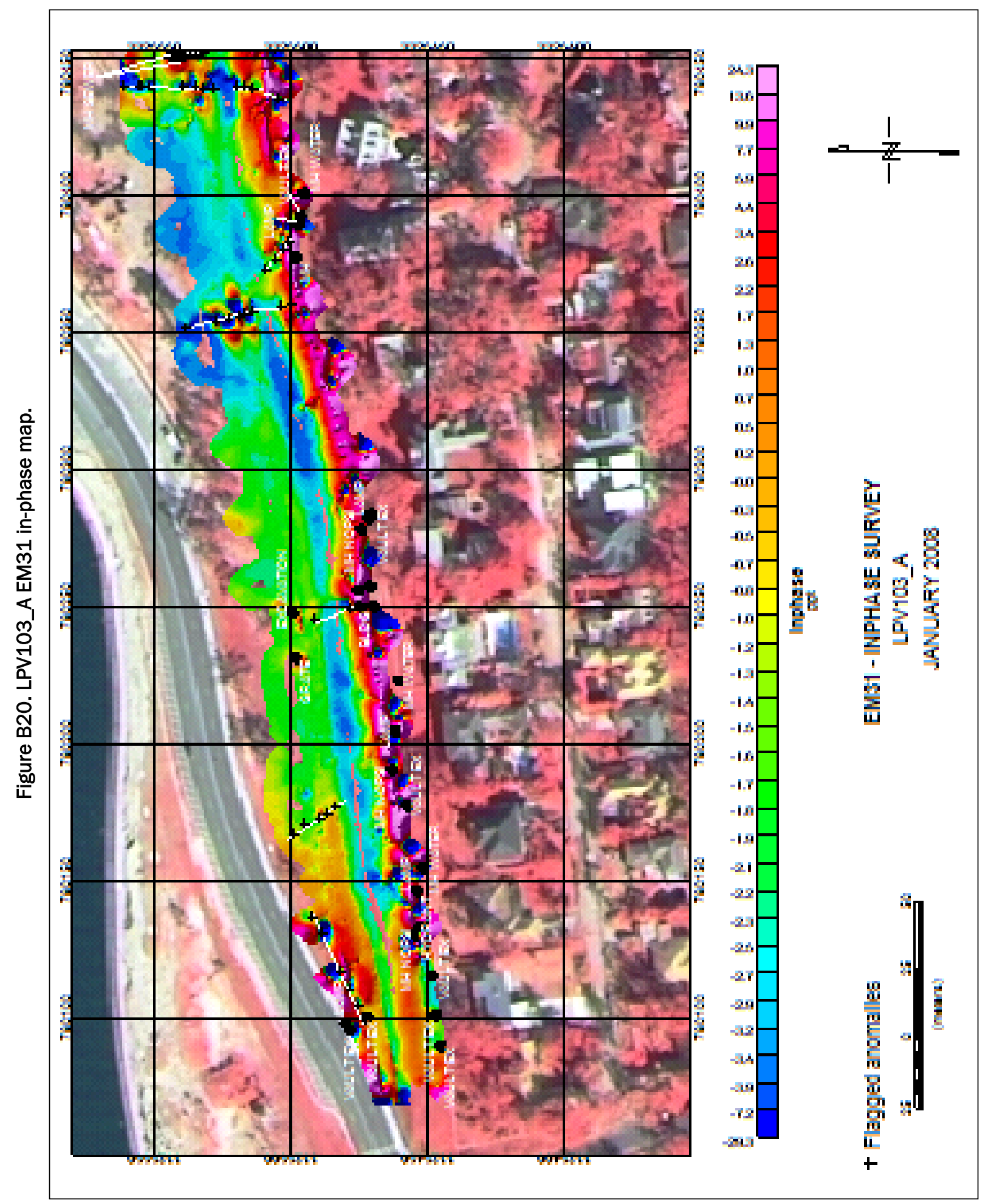




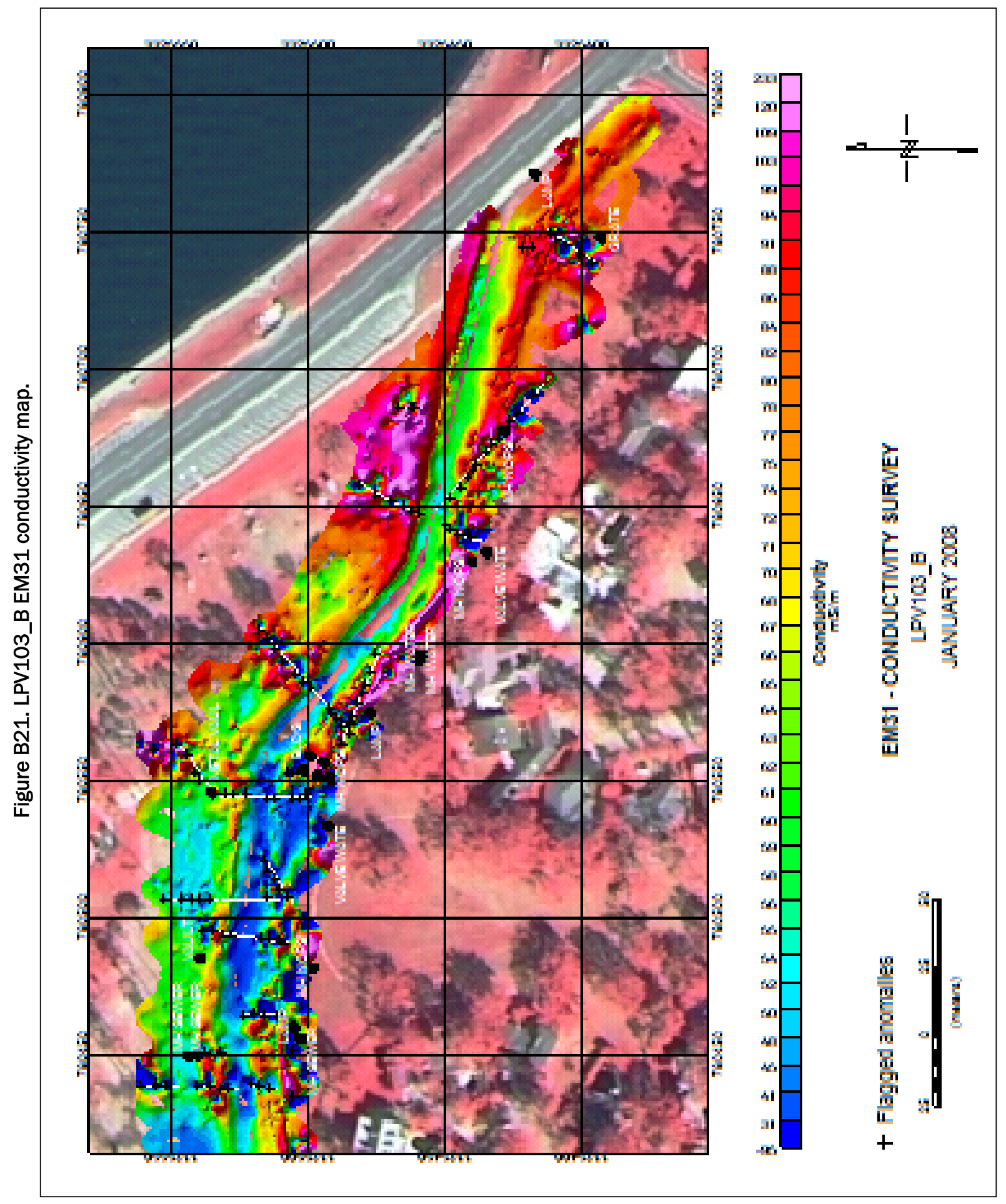




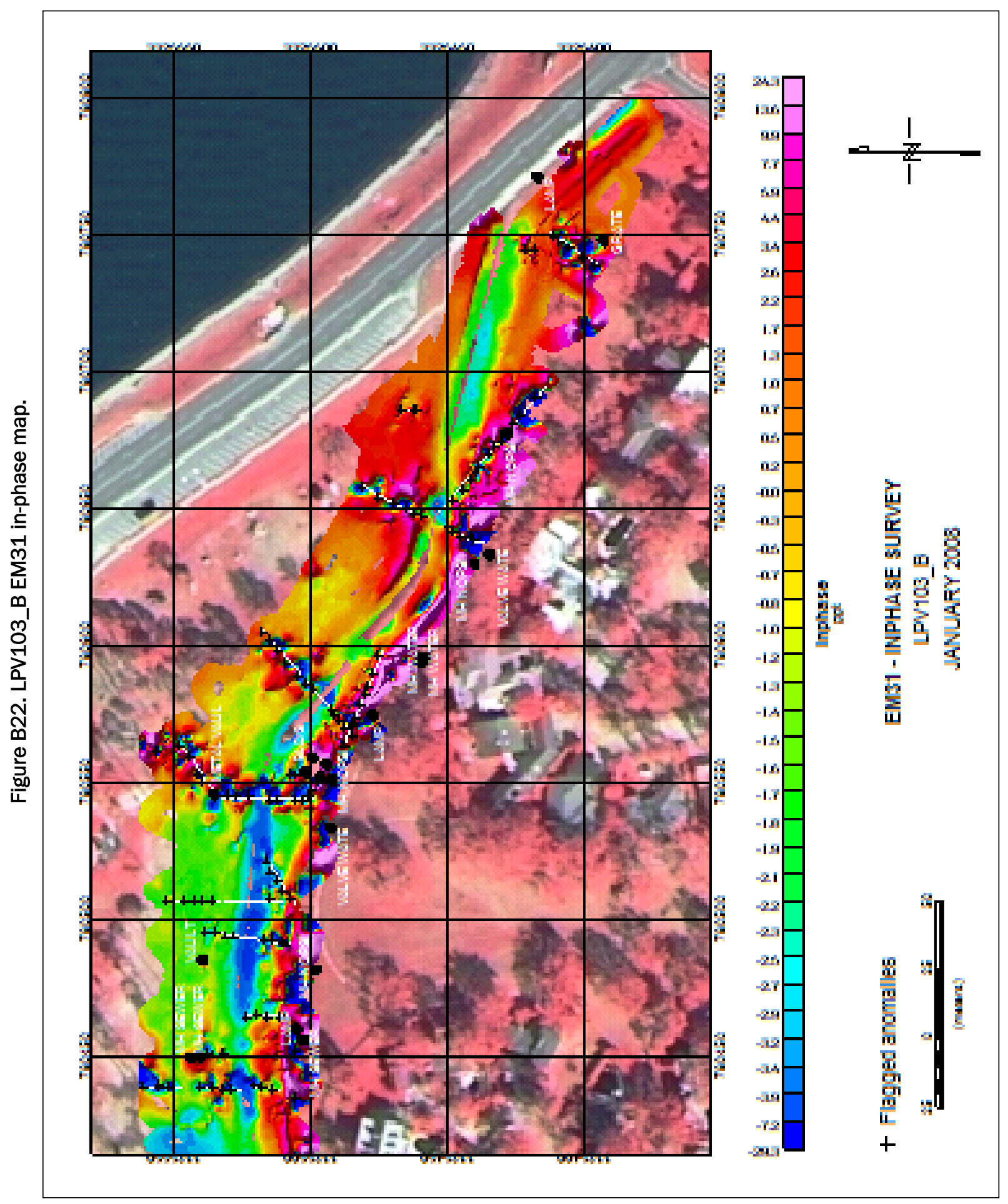




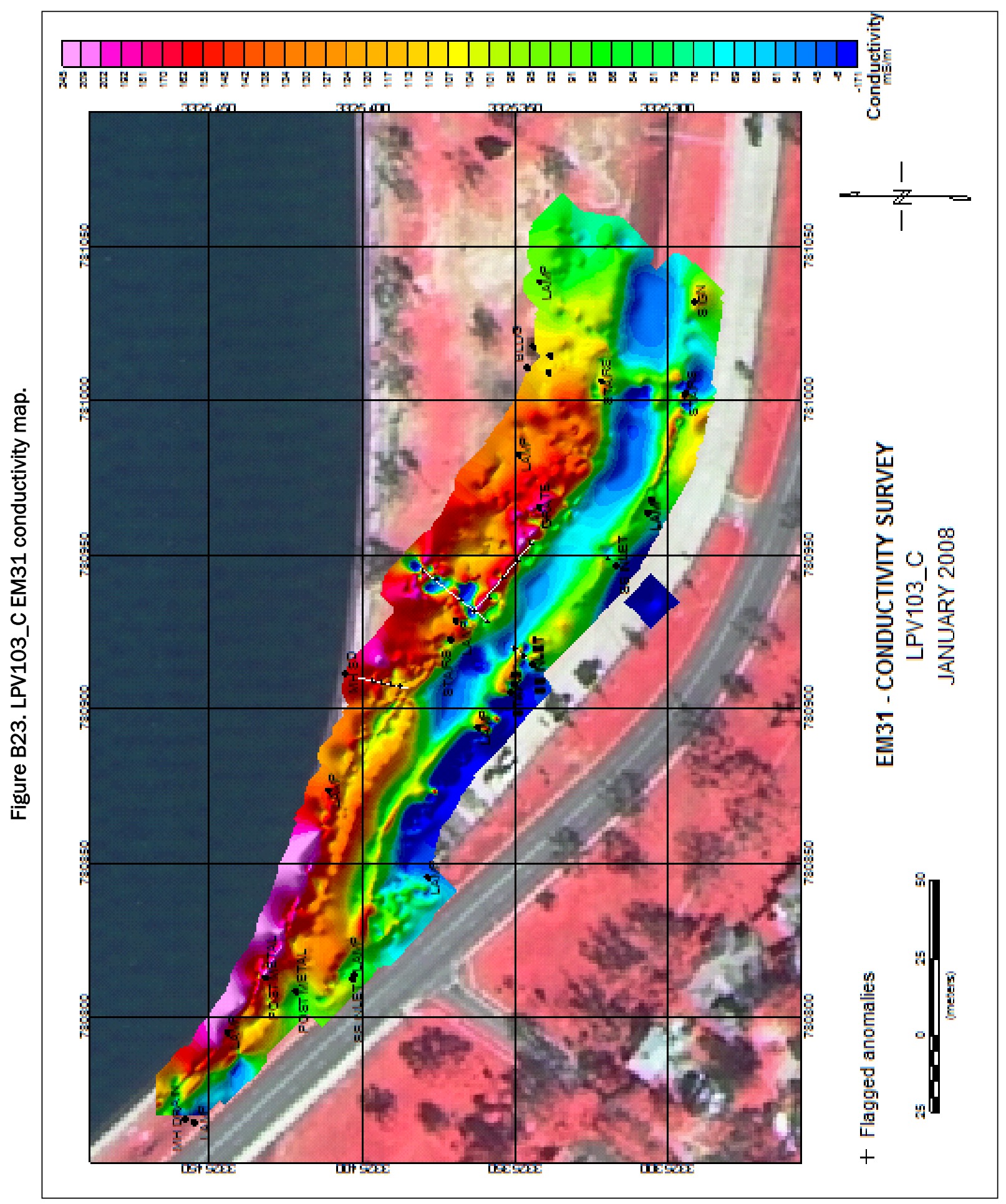




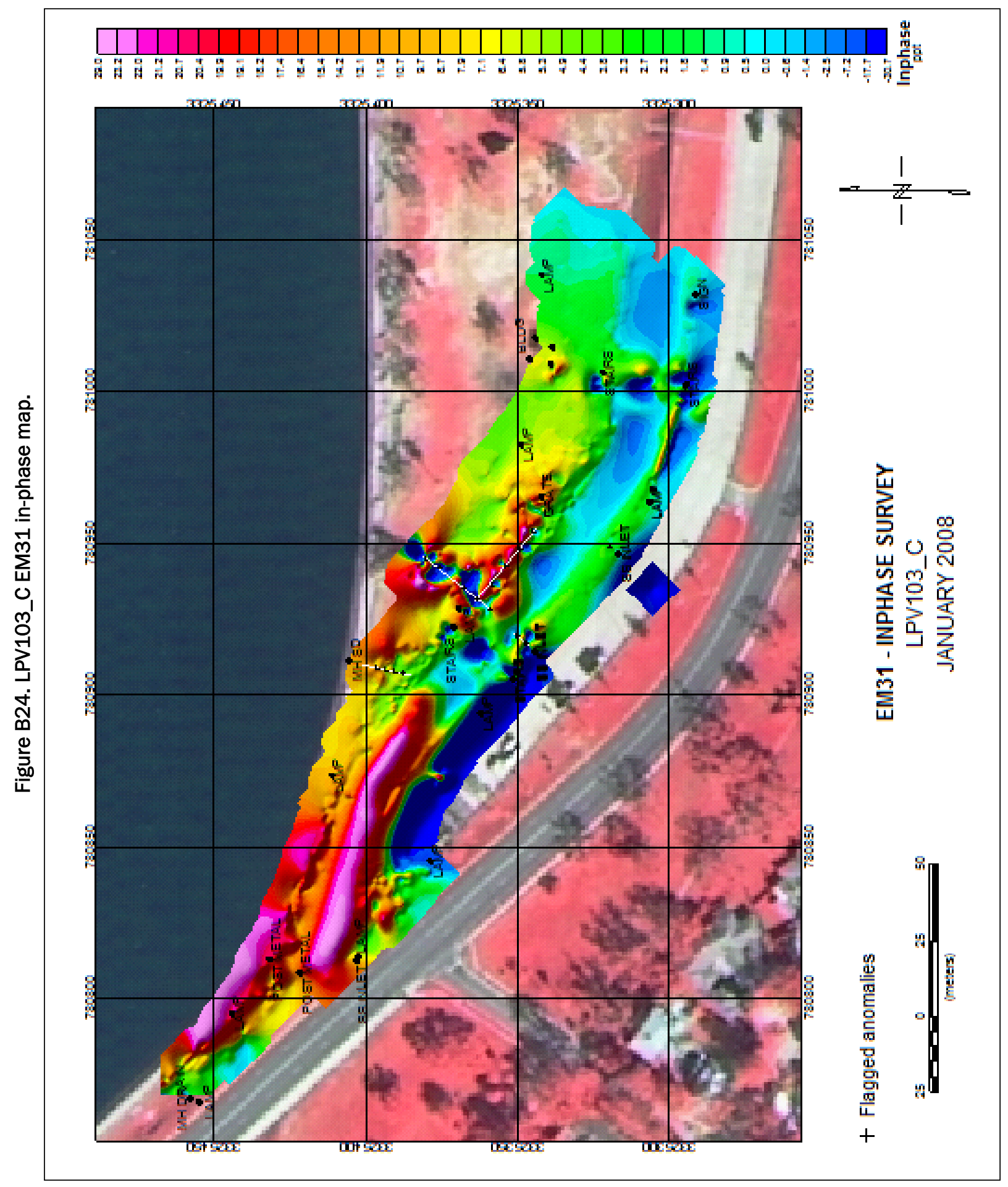


Figure B25. LPV103_D EM31 conductivity map.

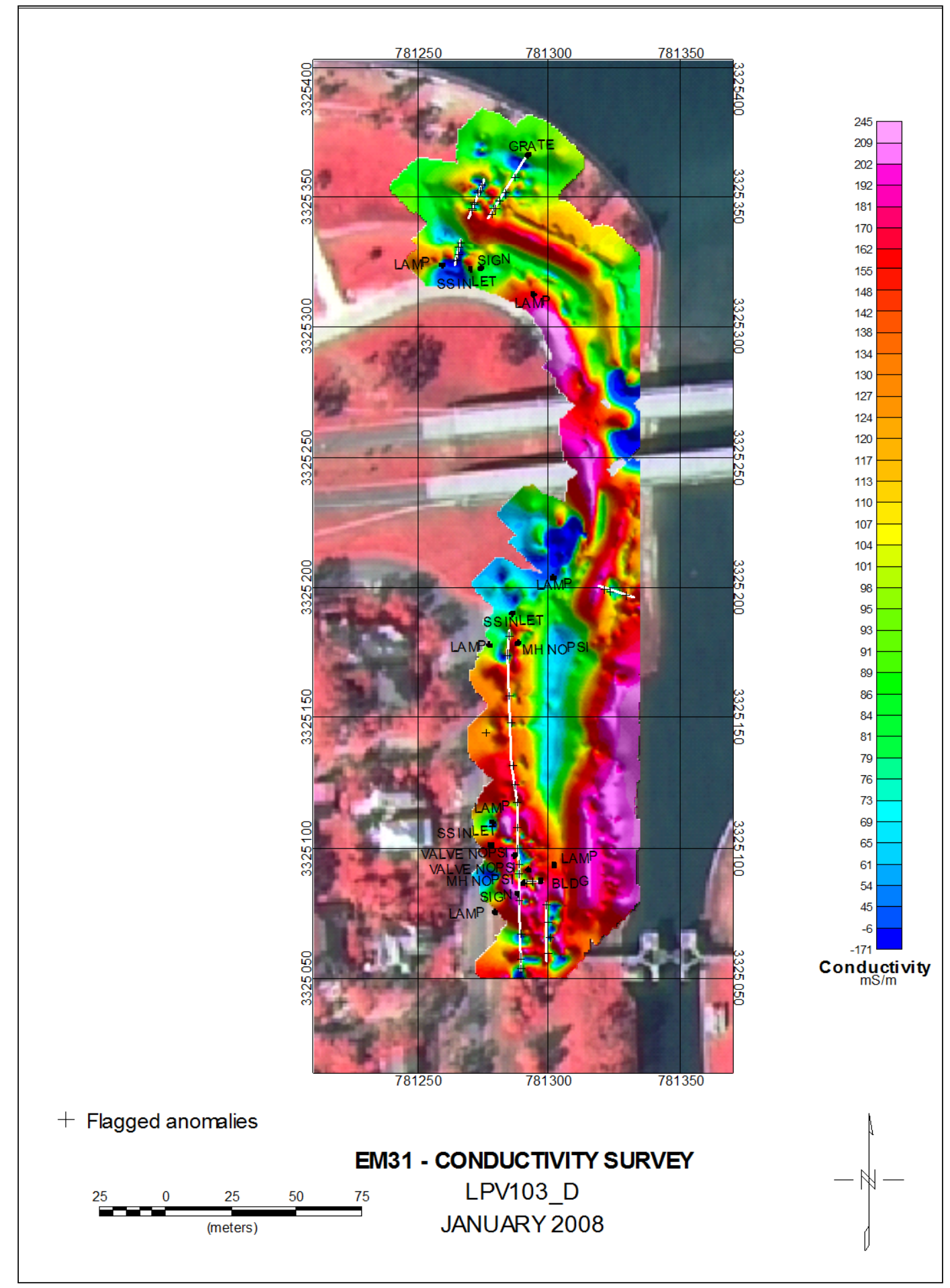


Figure B26. LPV103_D EM31 in-phase map.

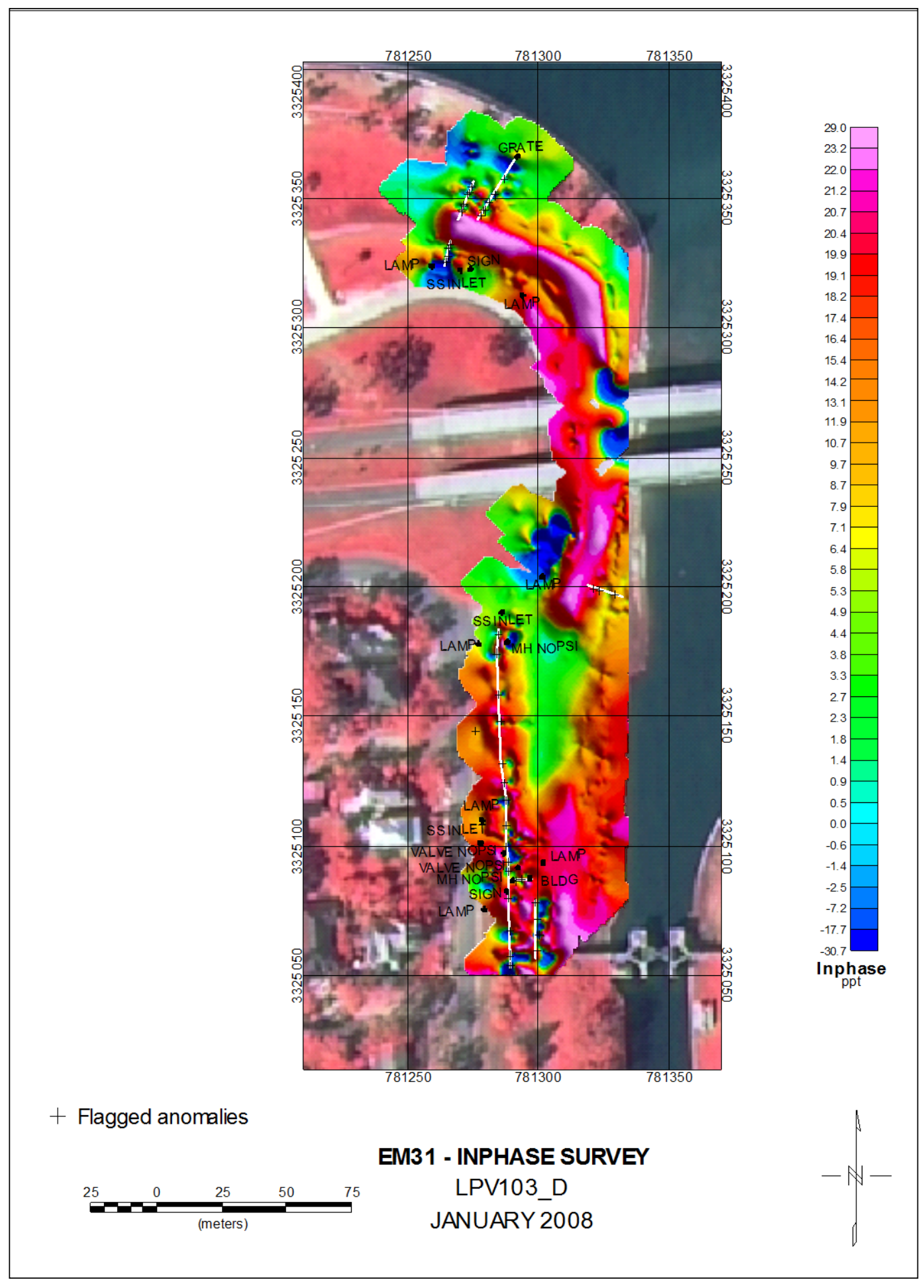


Figure B27. LPV103_E EM31 conductivity map.

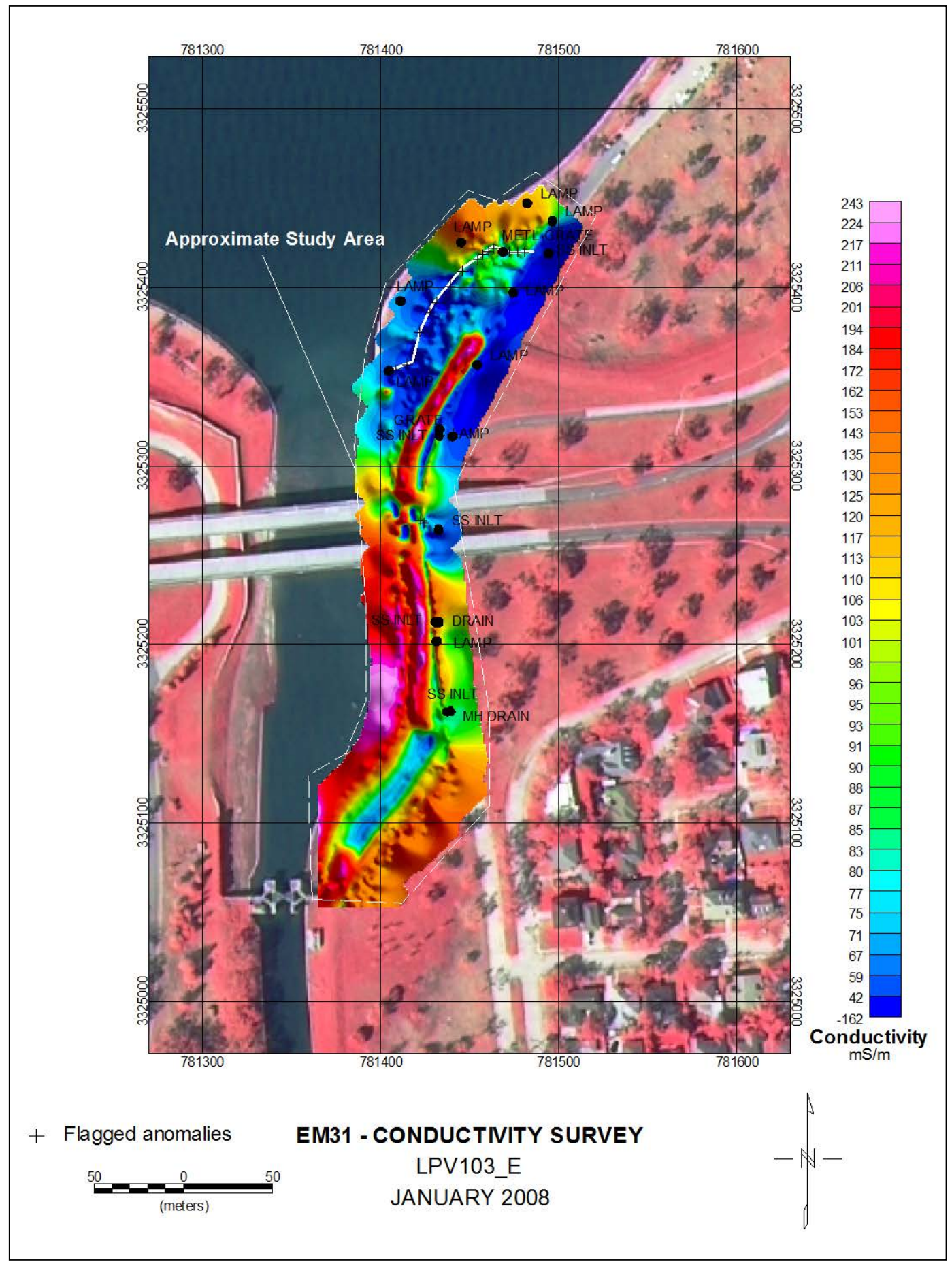


Figure B28. LPV103_E EM31 in-phase map.

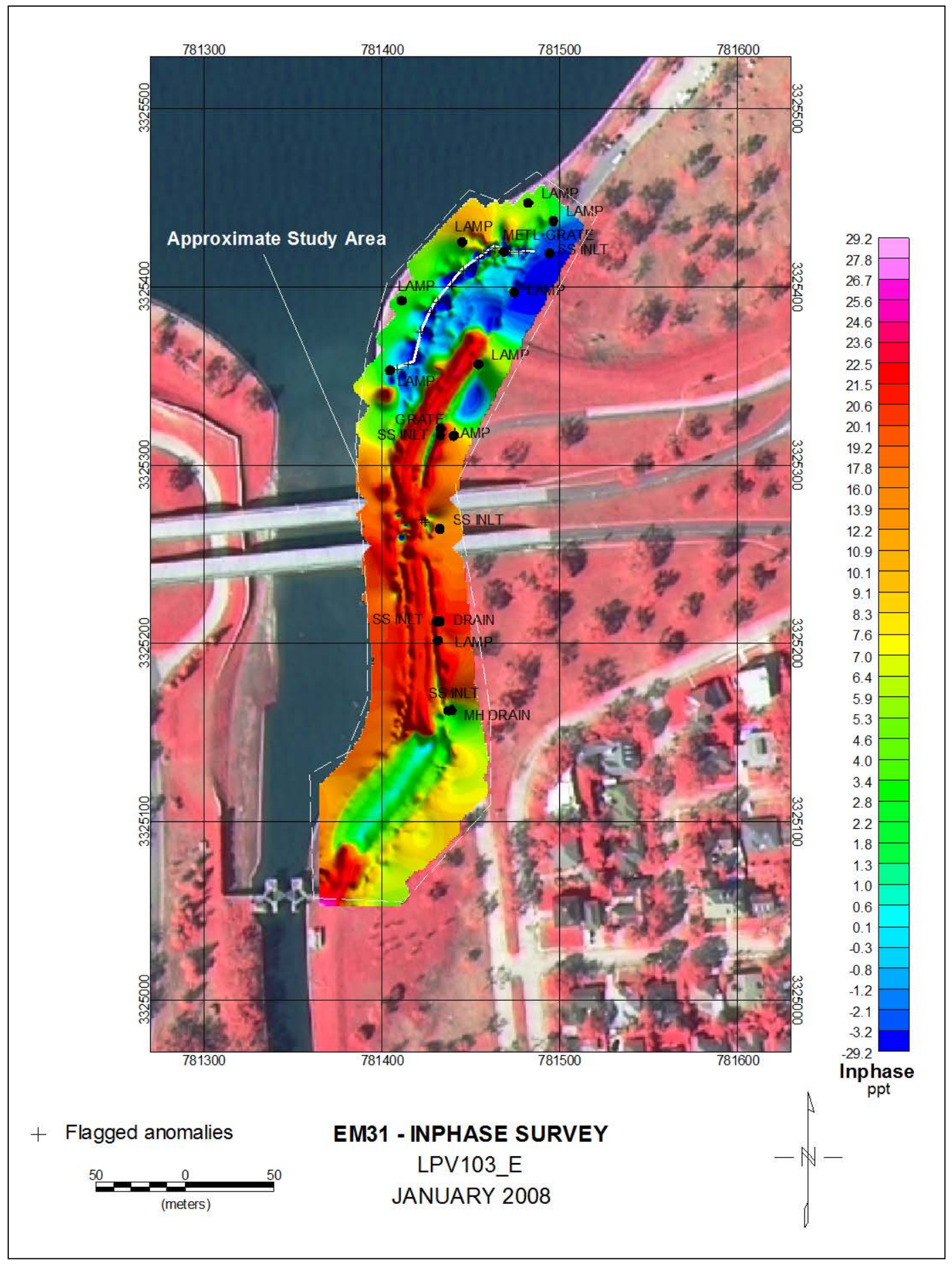




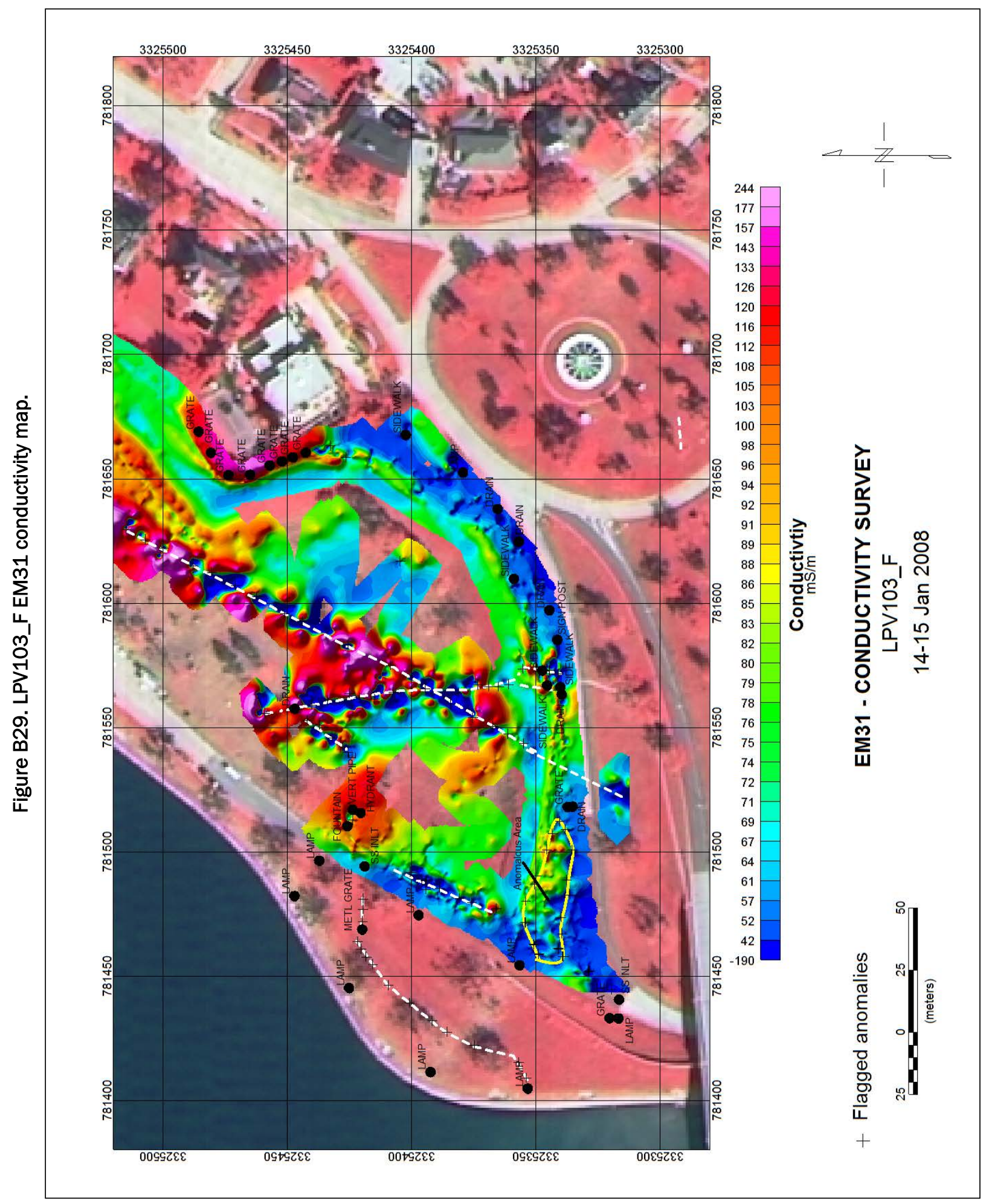




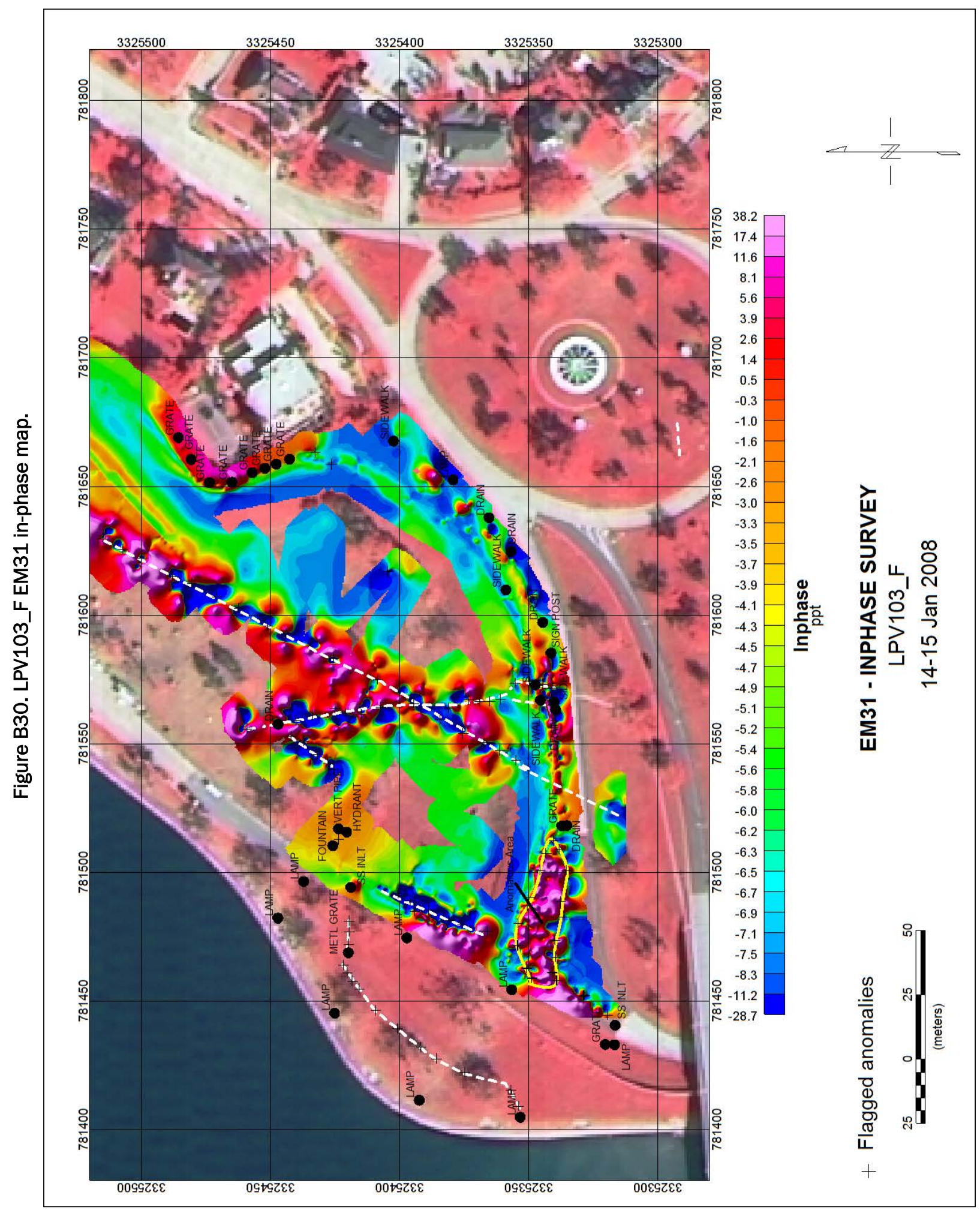




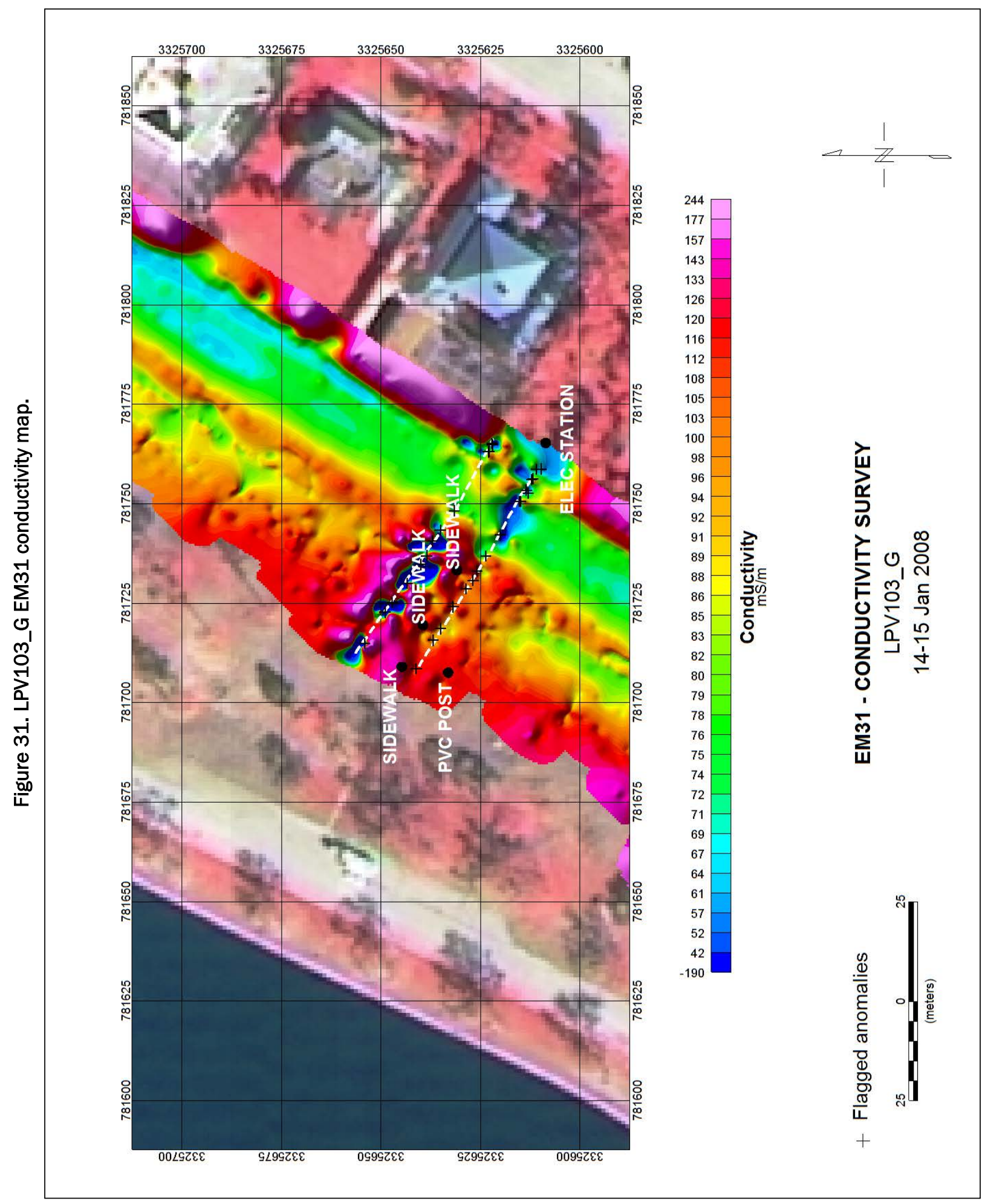




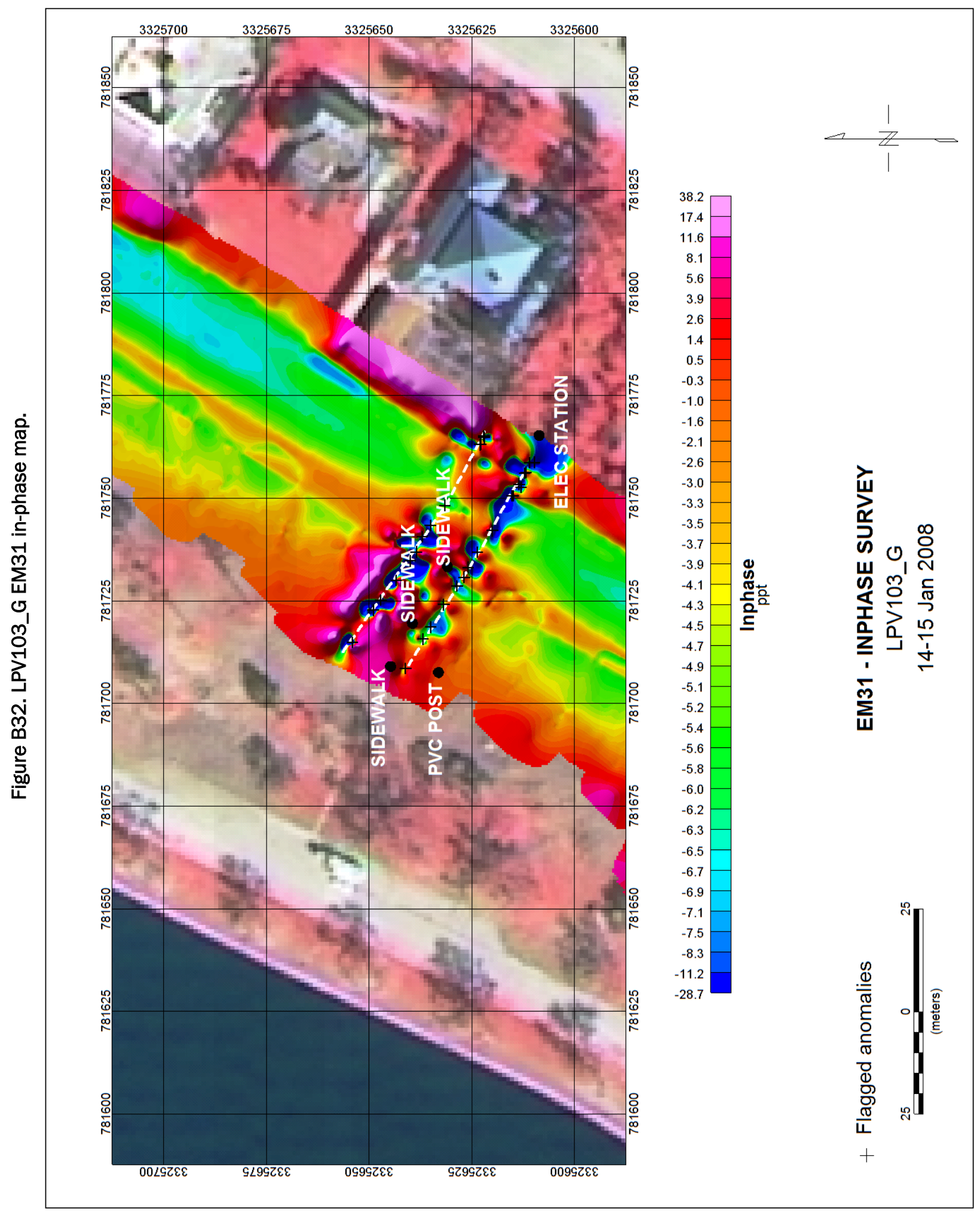




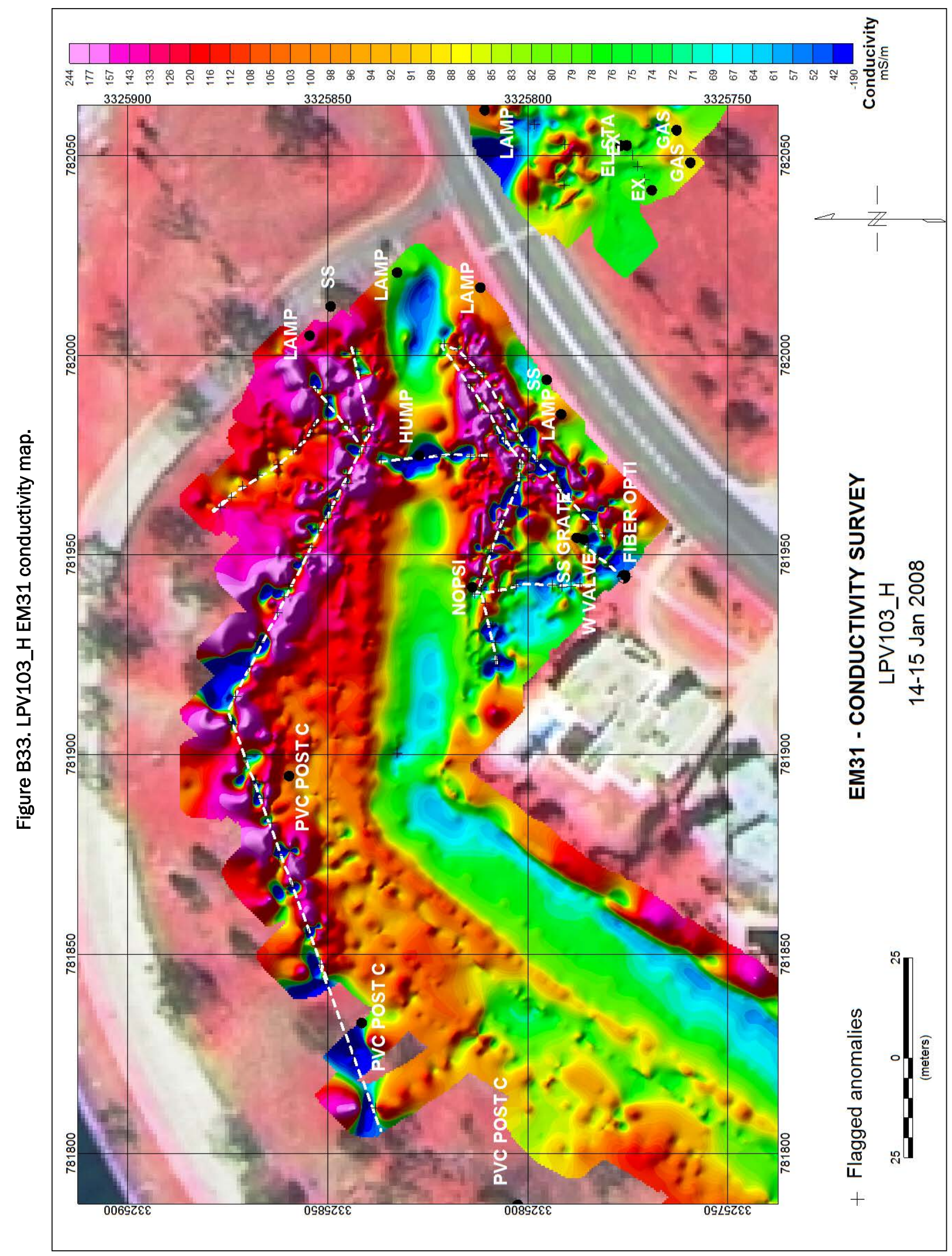




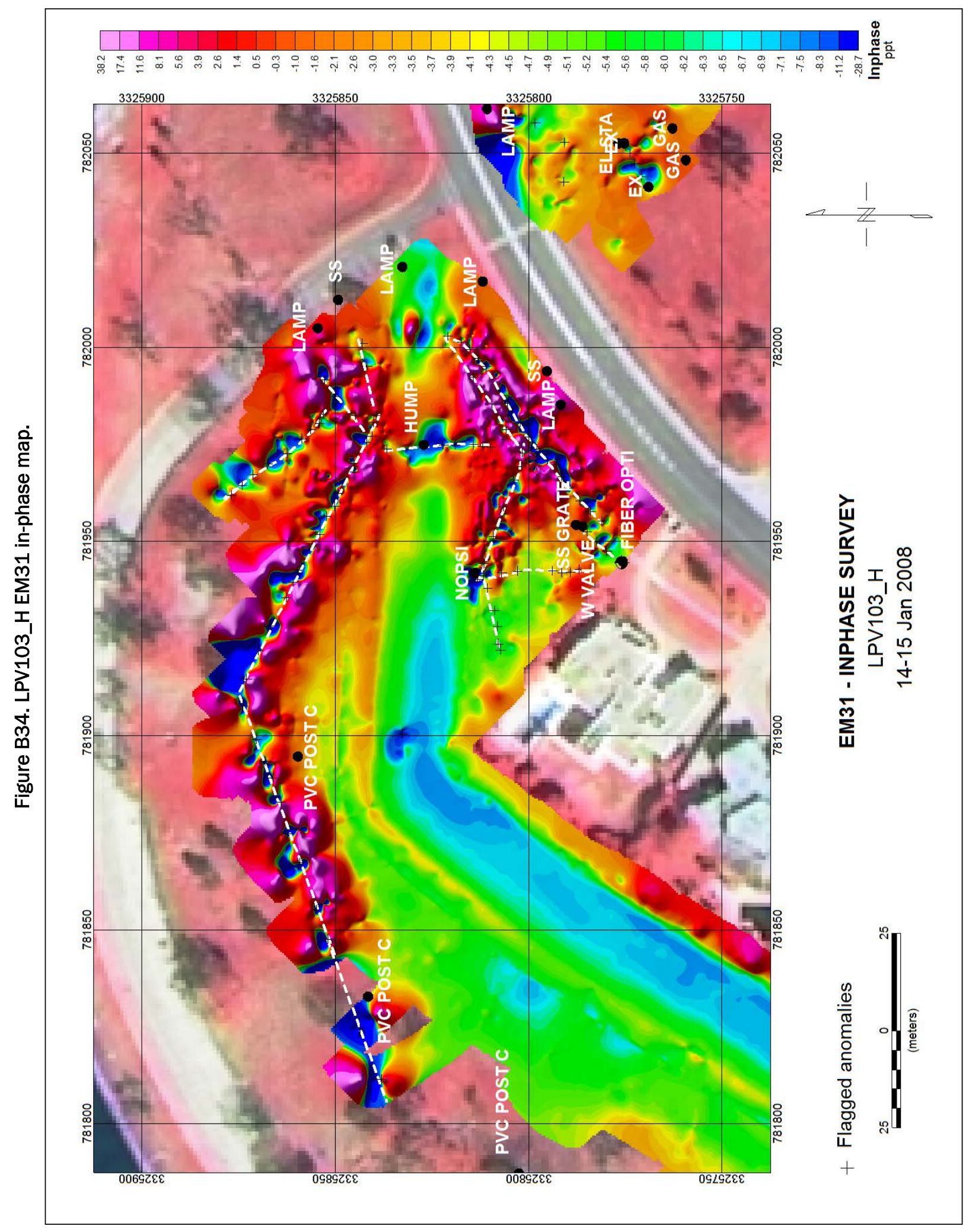




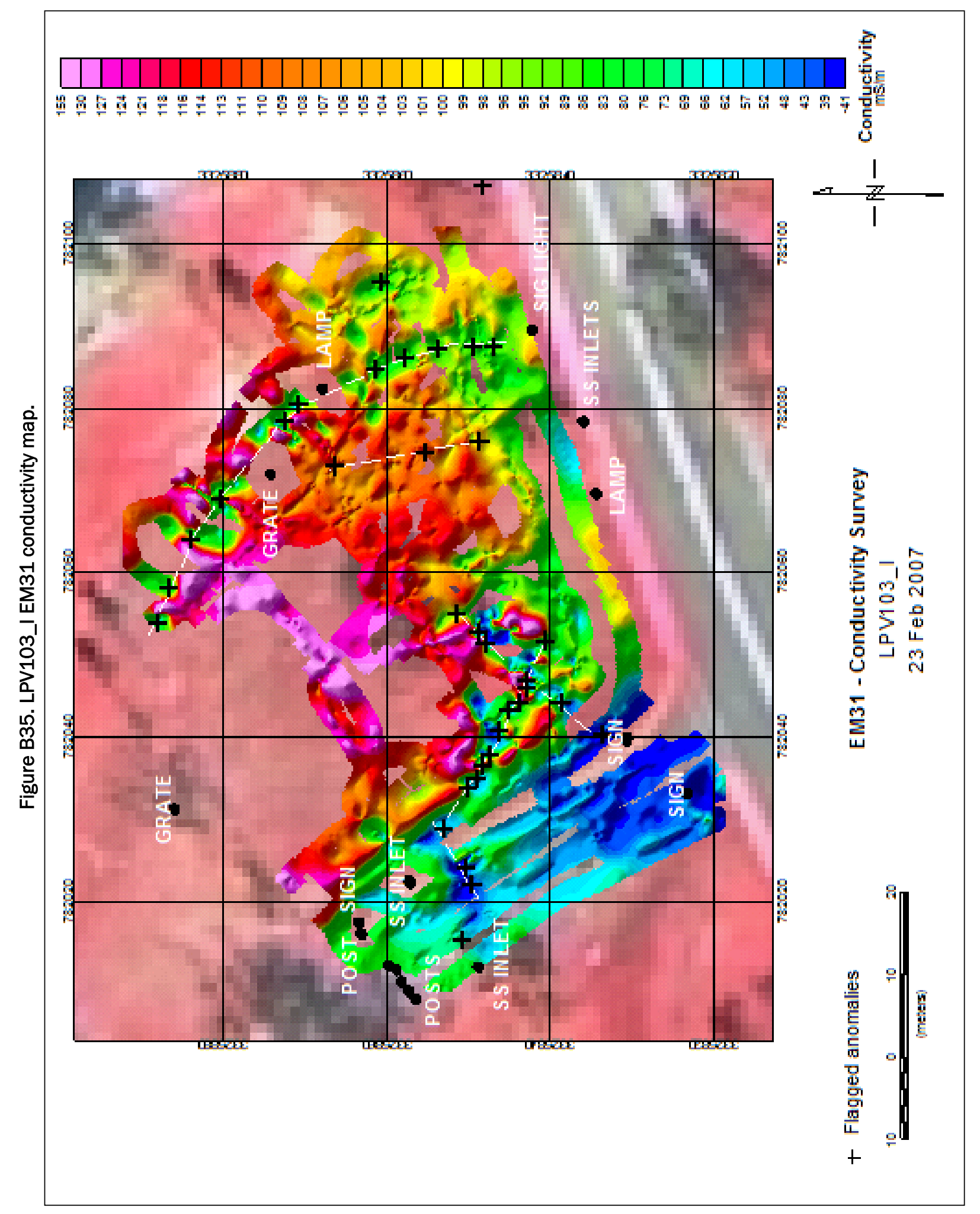




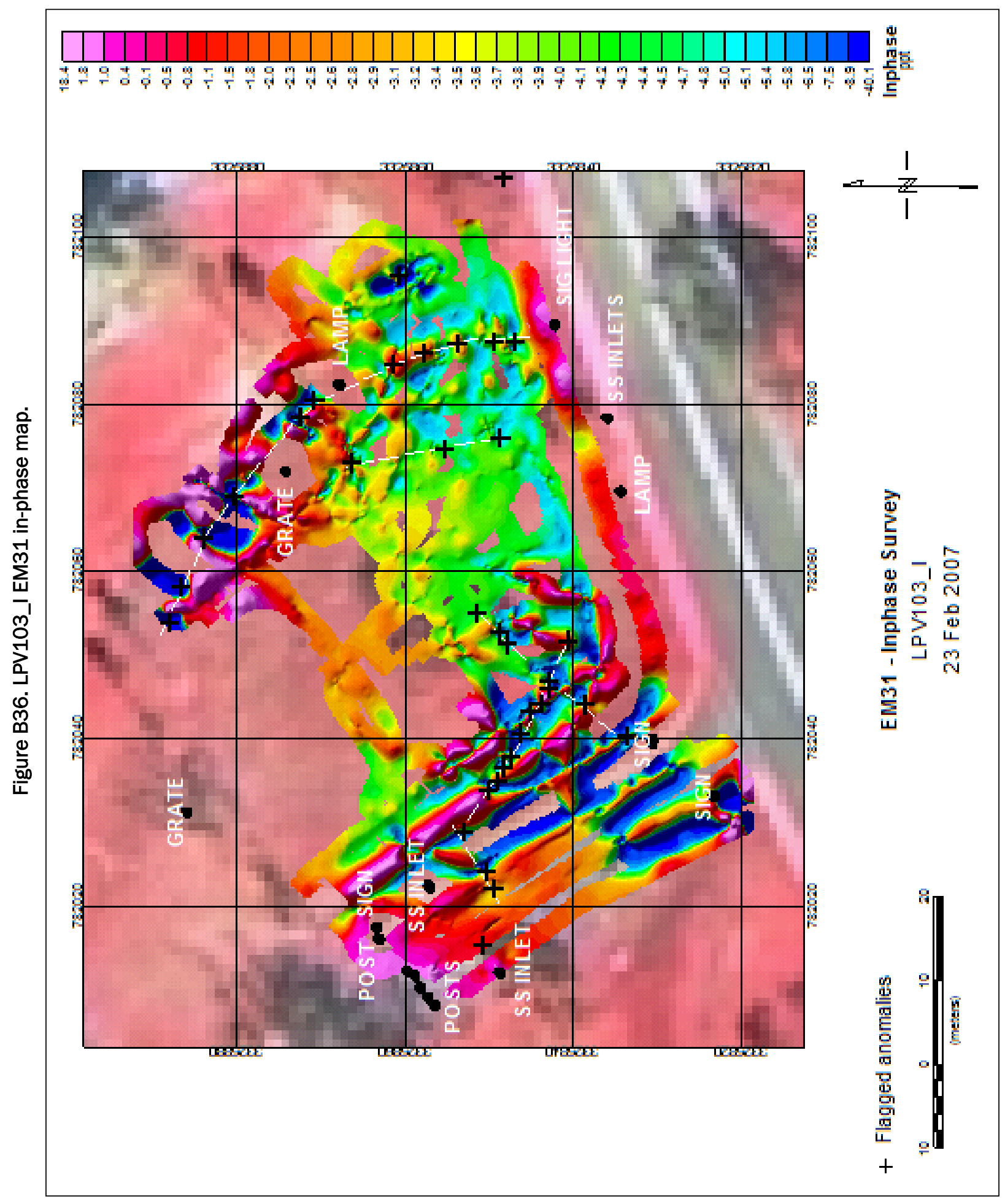




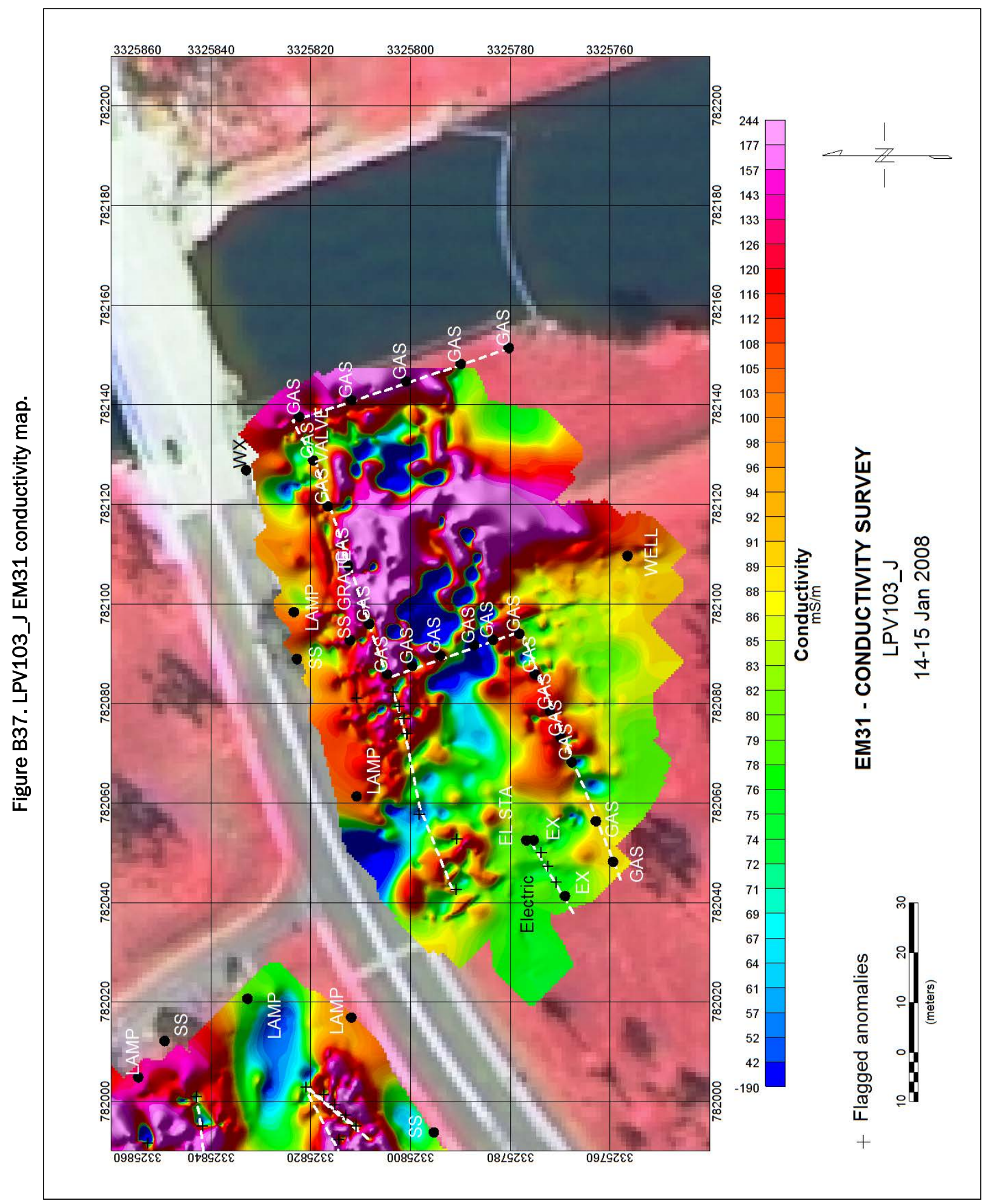




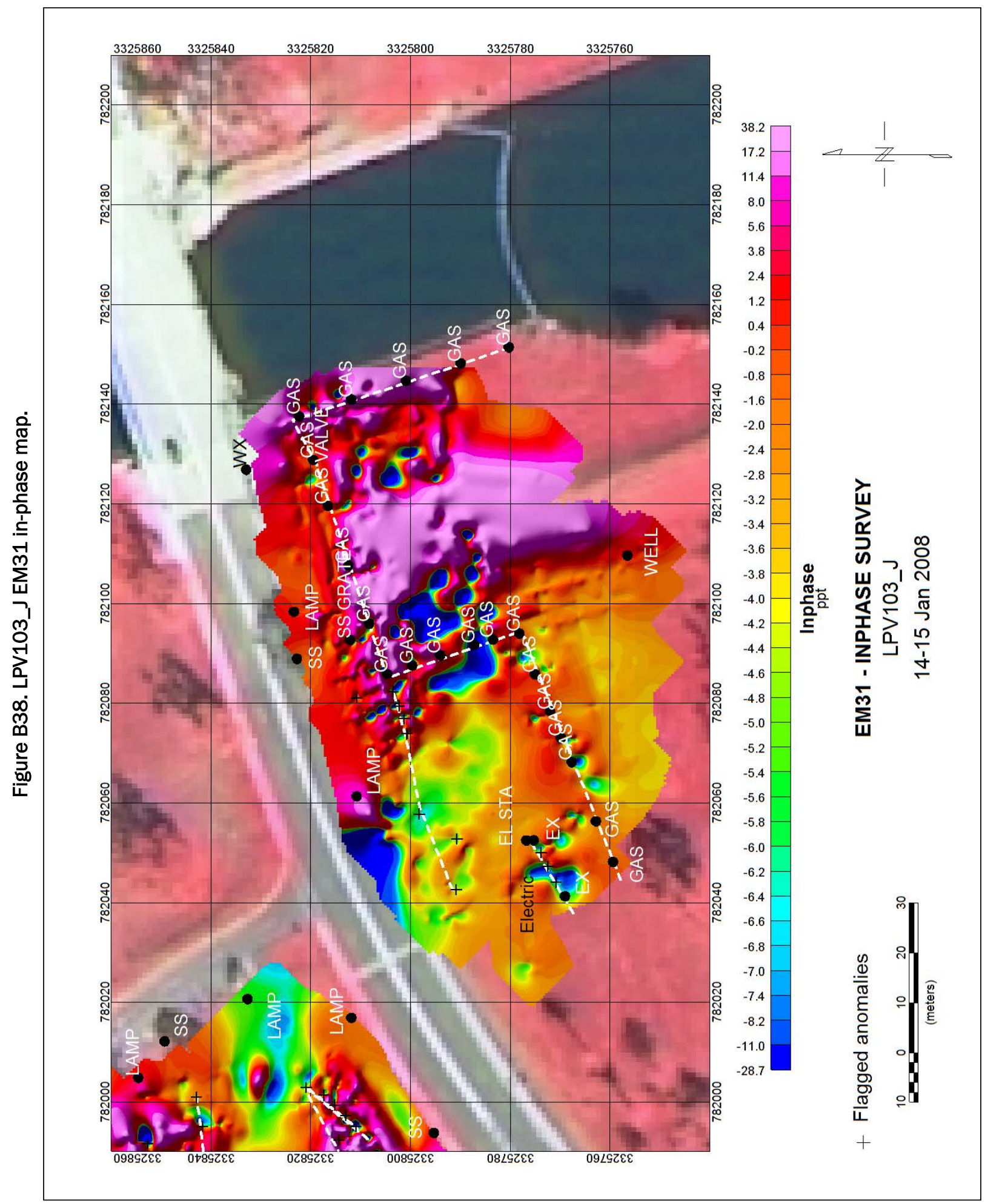


Figure B39. LPV103_K and LPV104_A EM31 conductivity map.

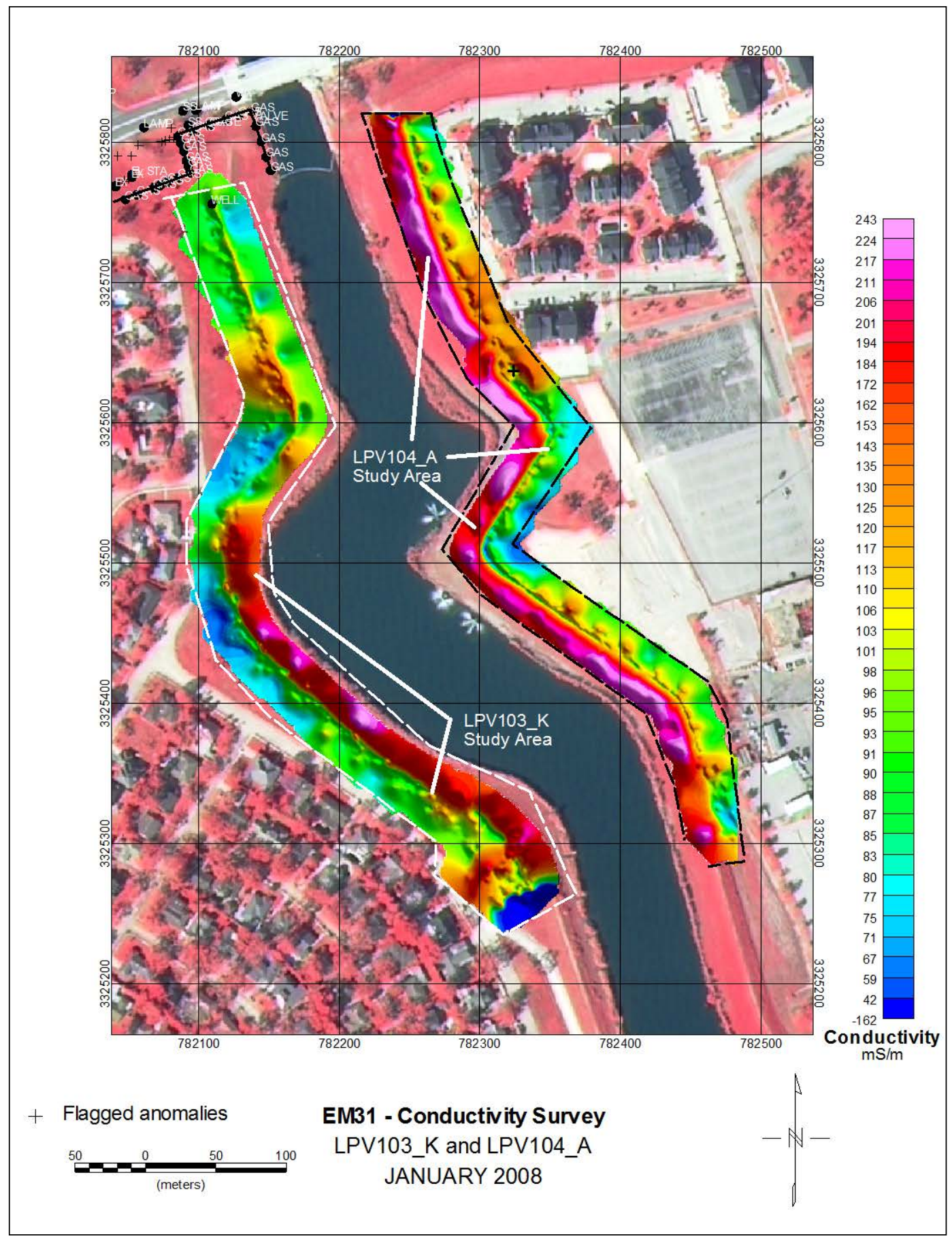


Figure B 40. LPV103_K and LPV104_A EM31 in-phase map.

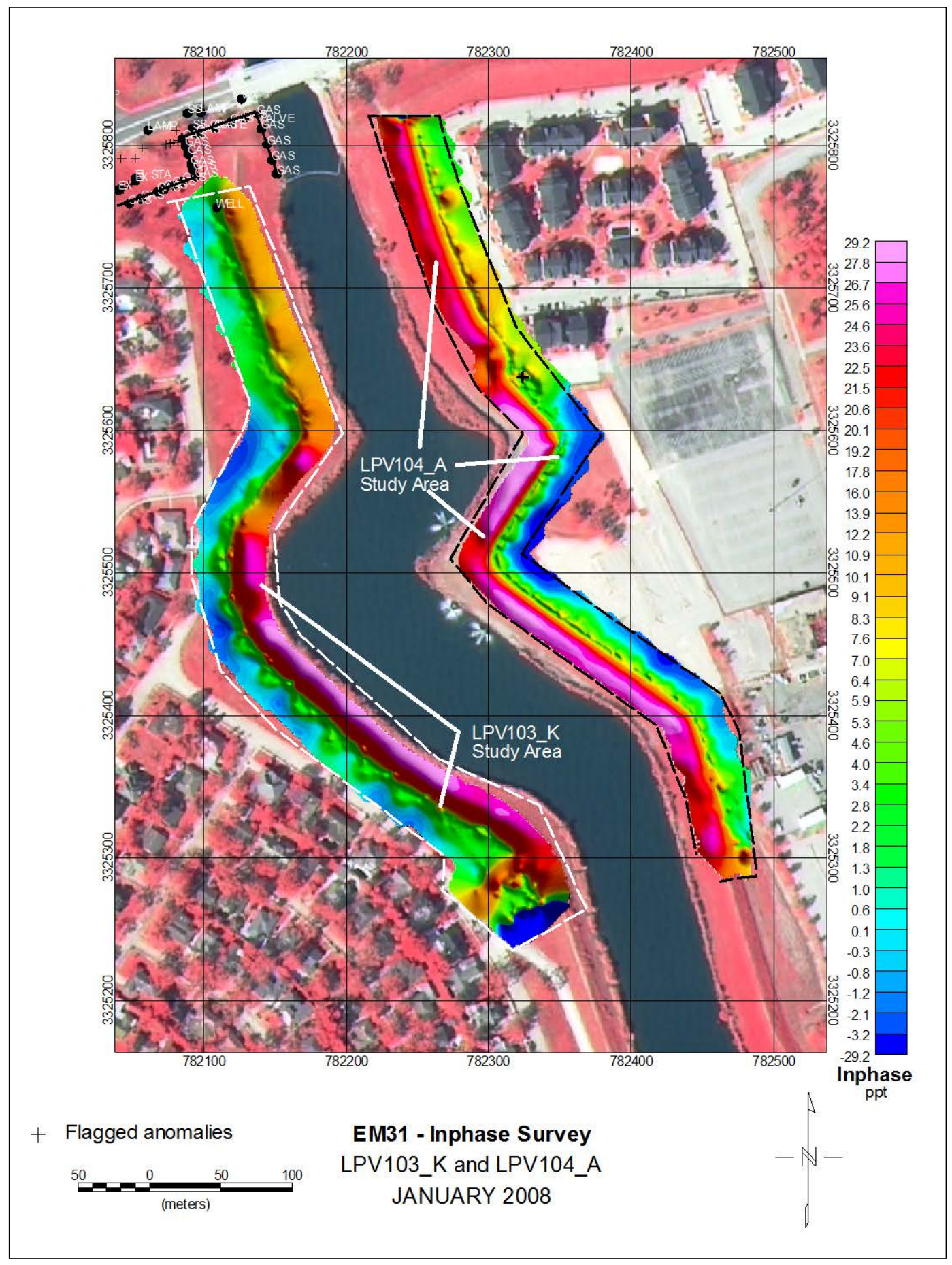




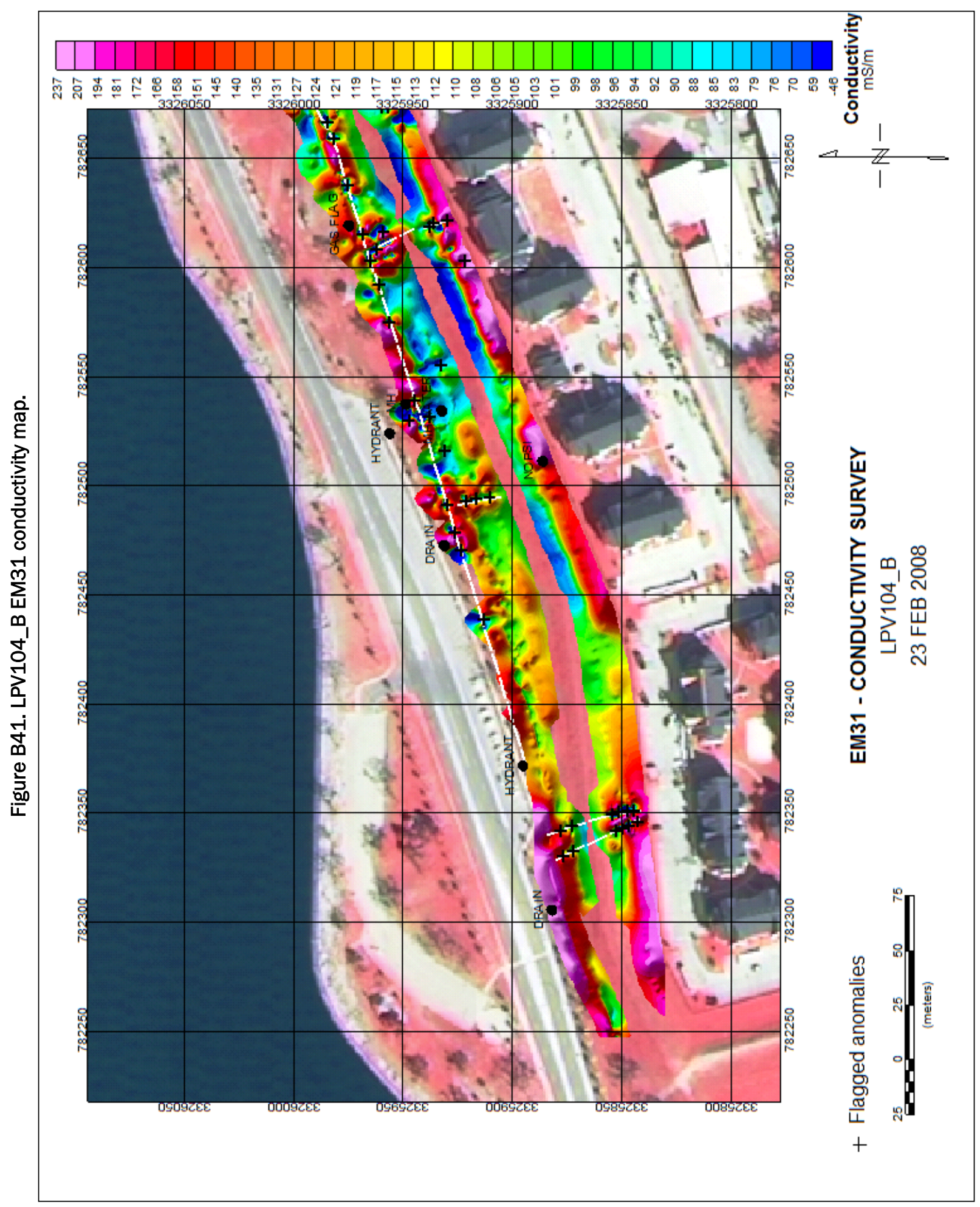




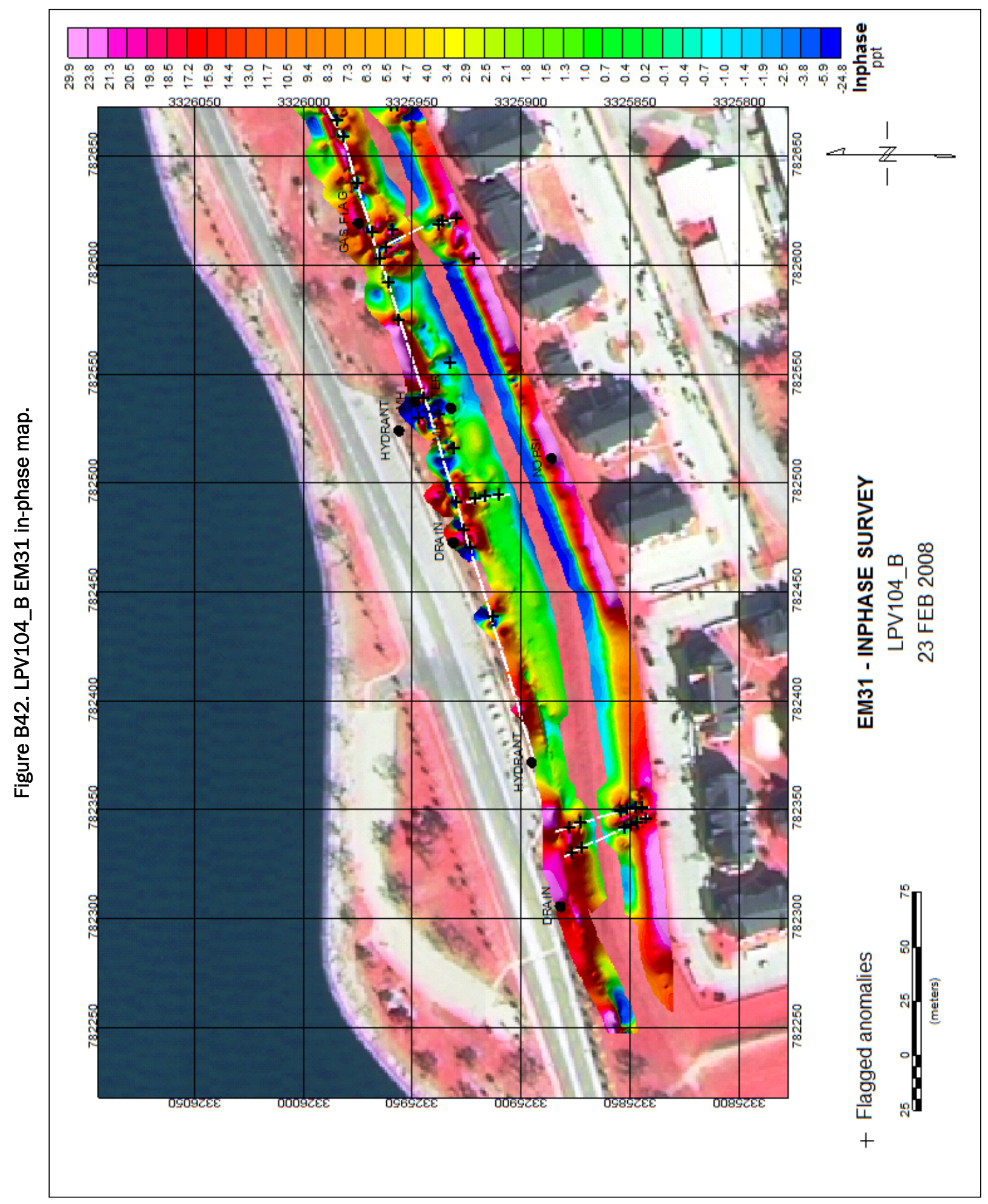




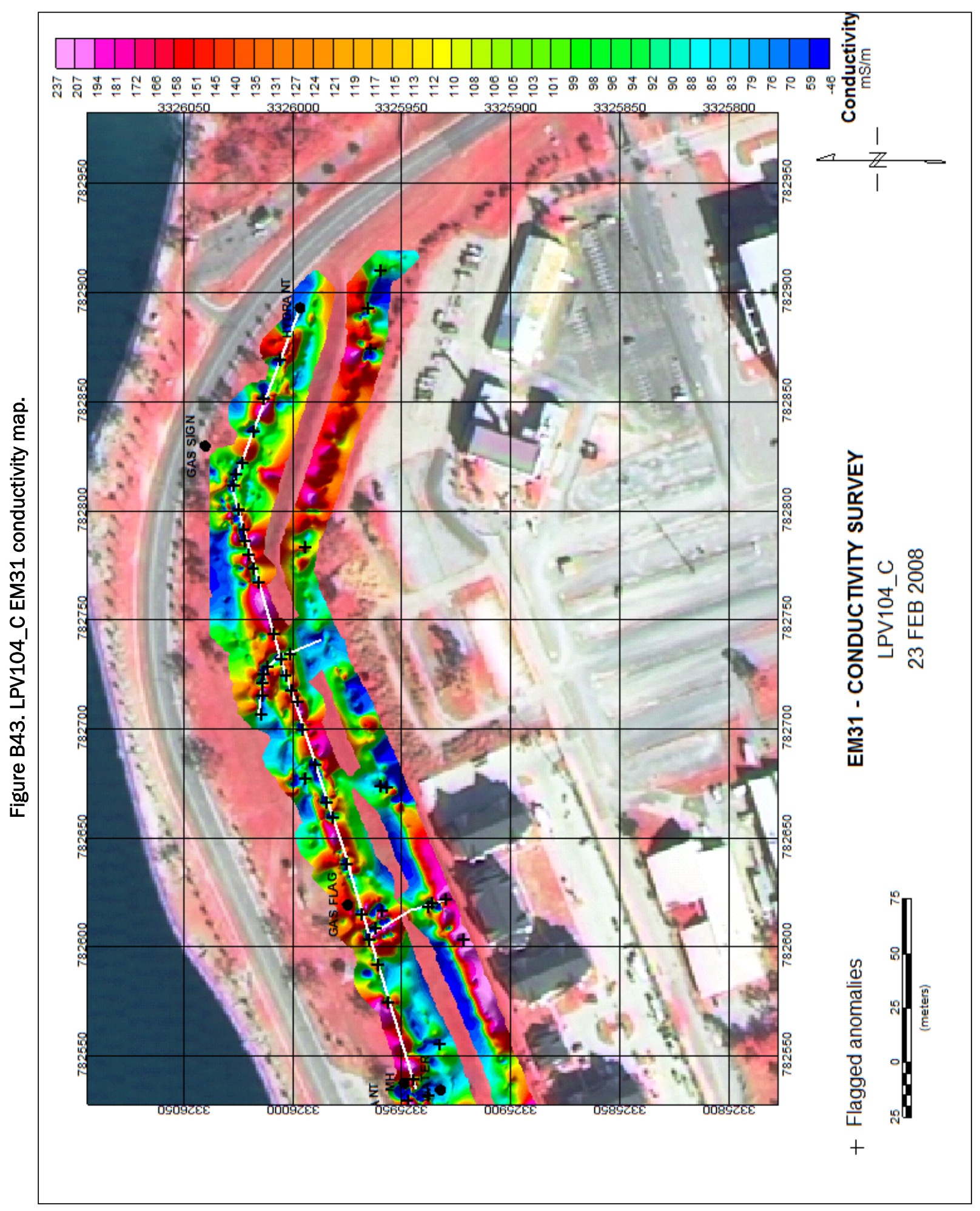




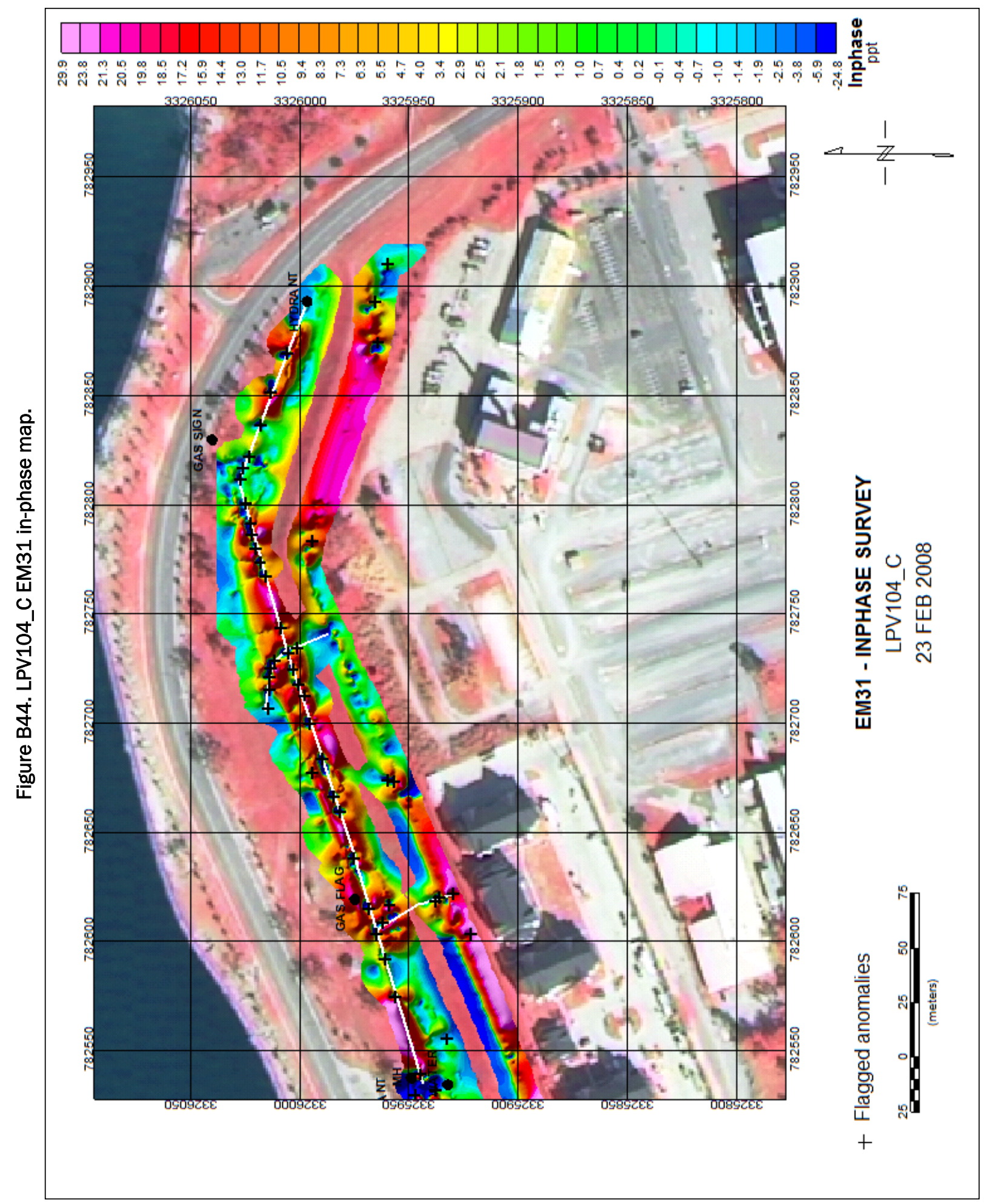




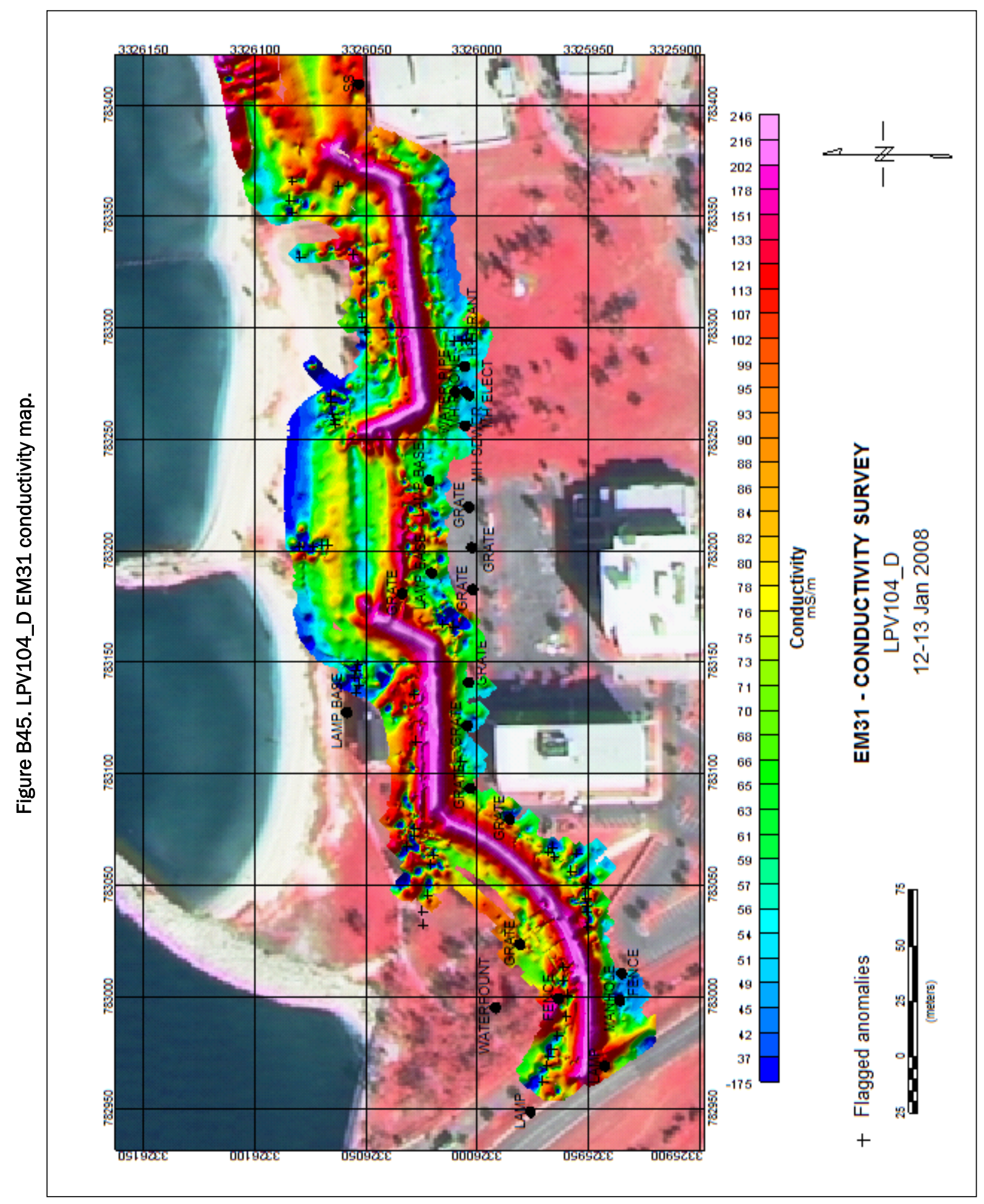




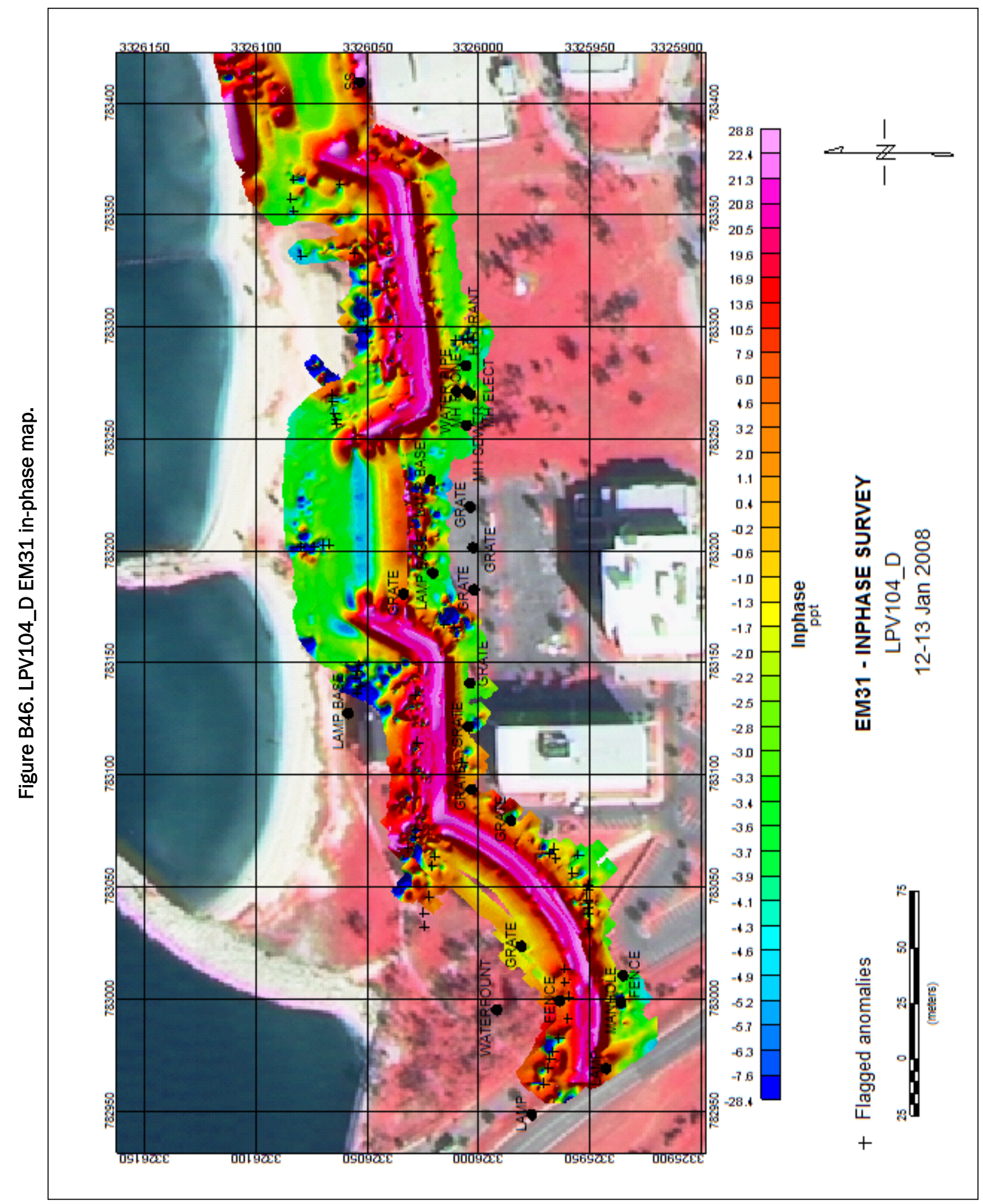




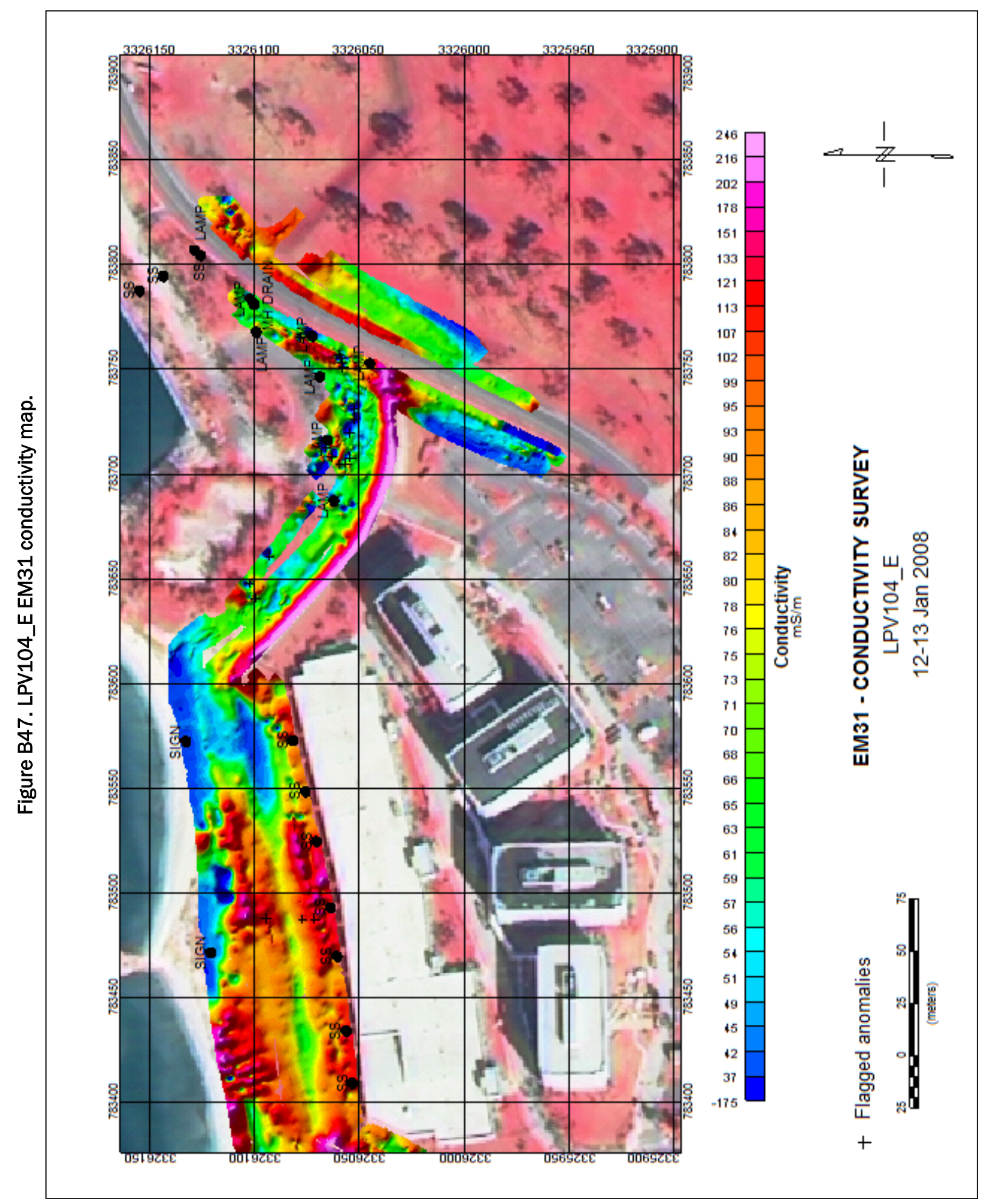




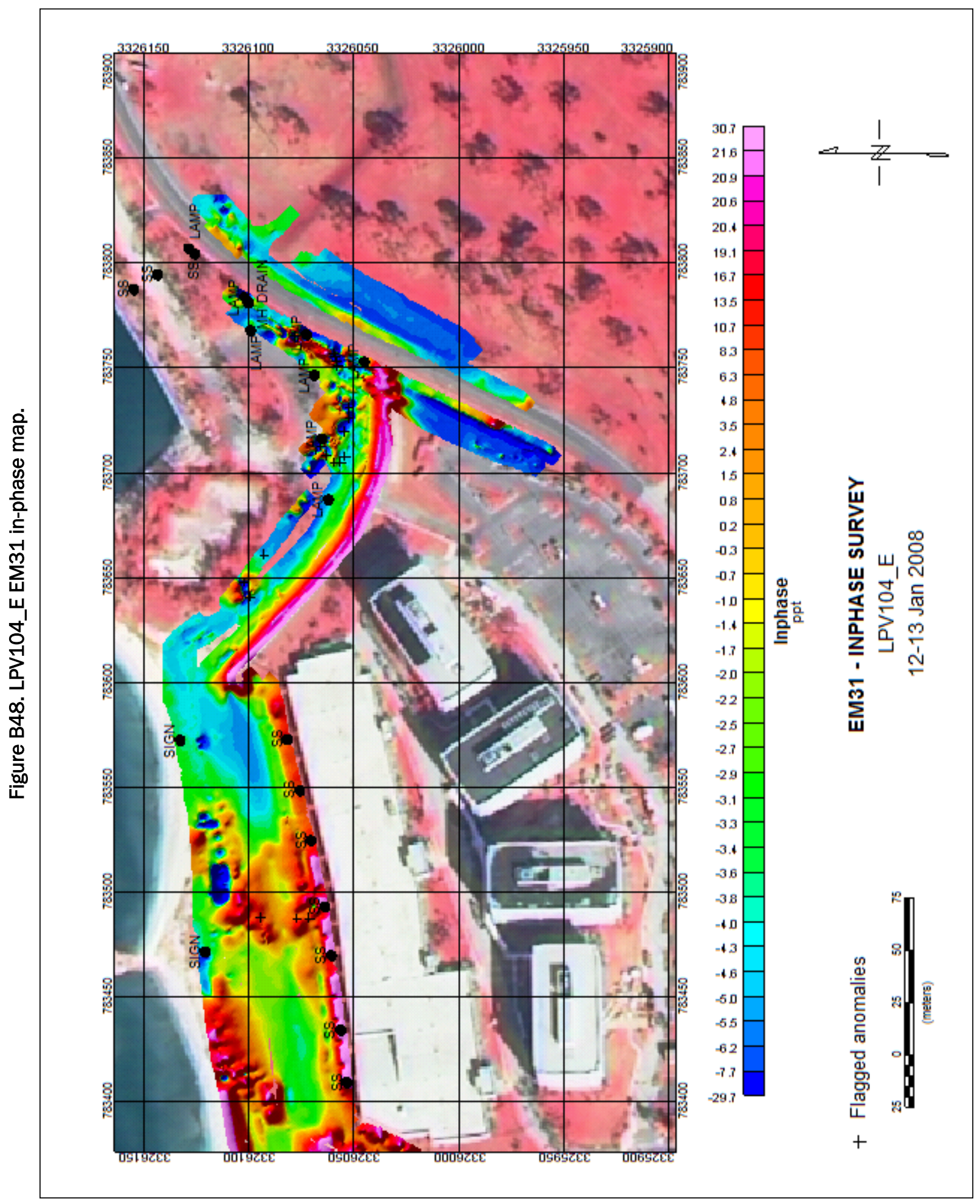




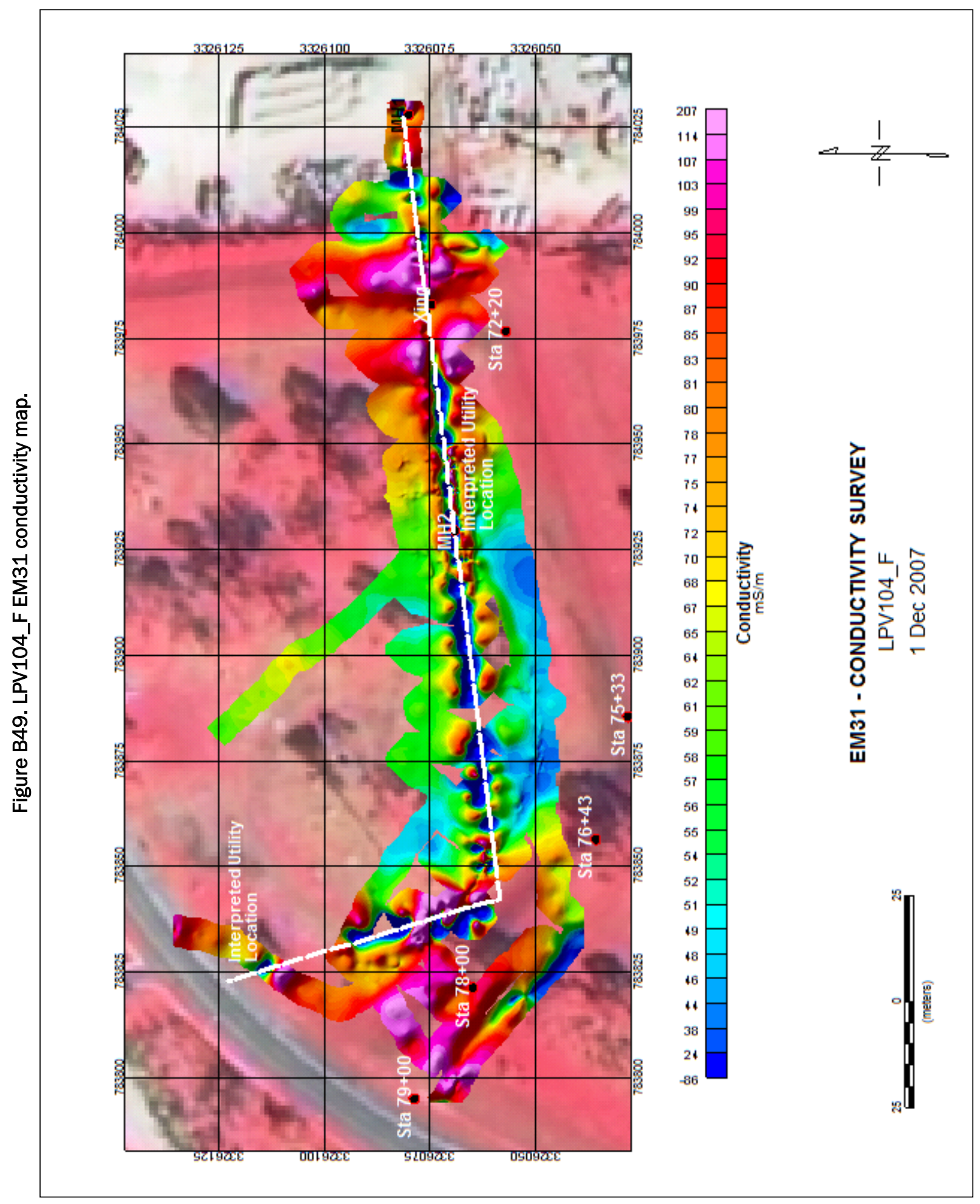




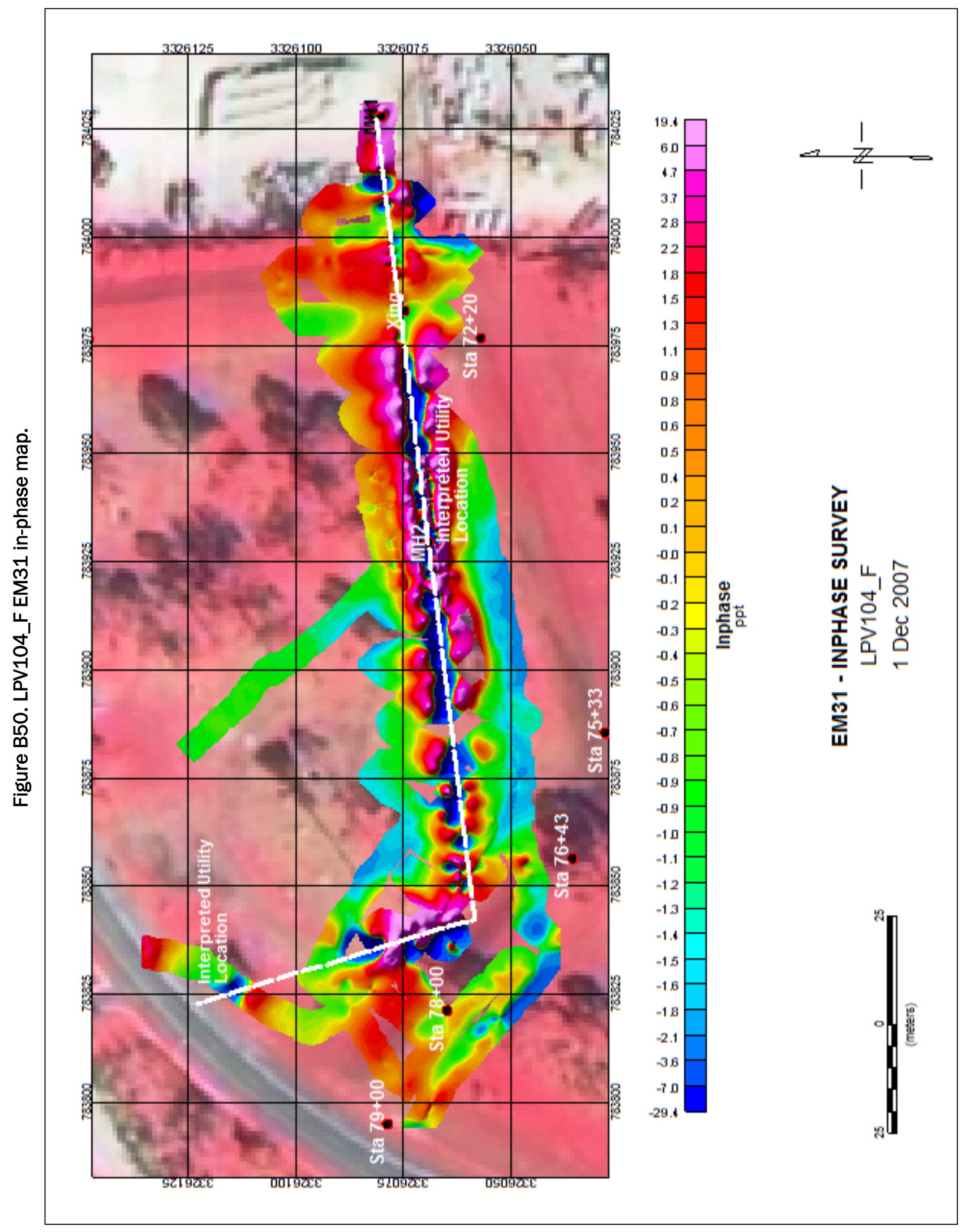




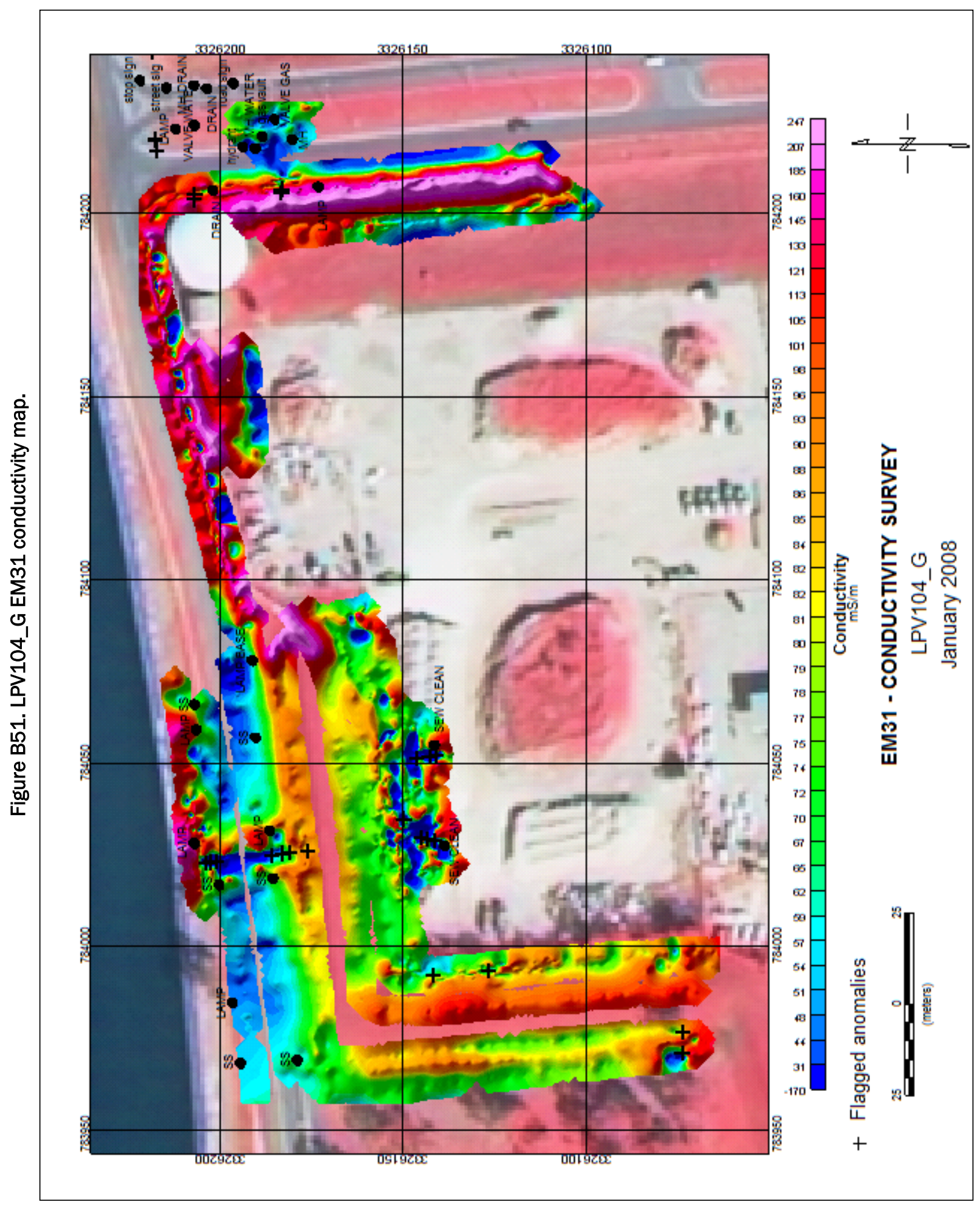




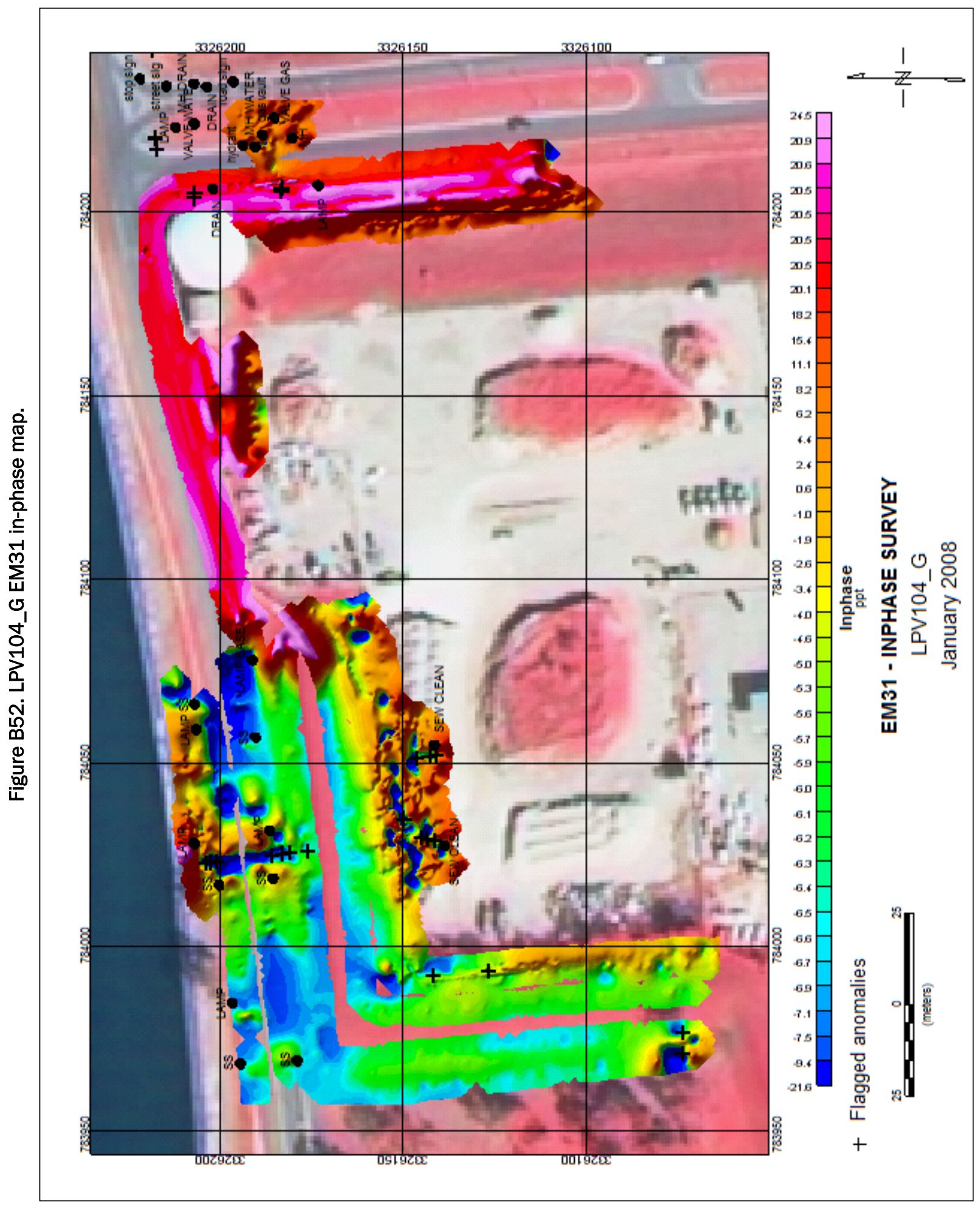




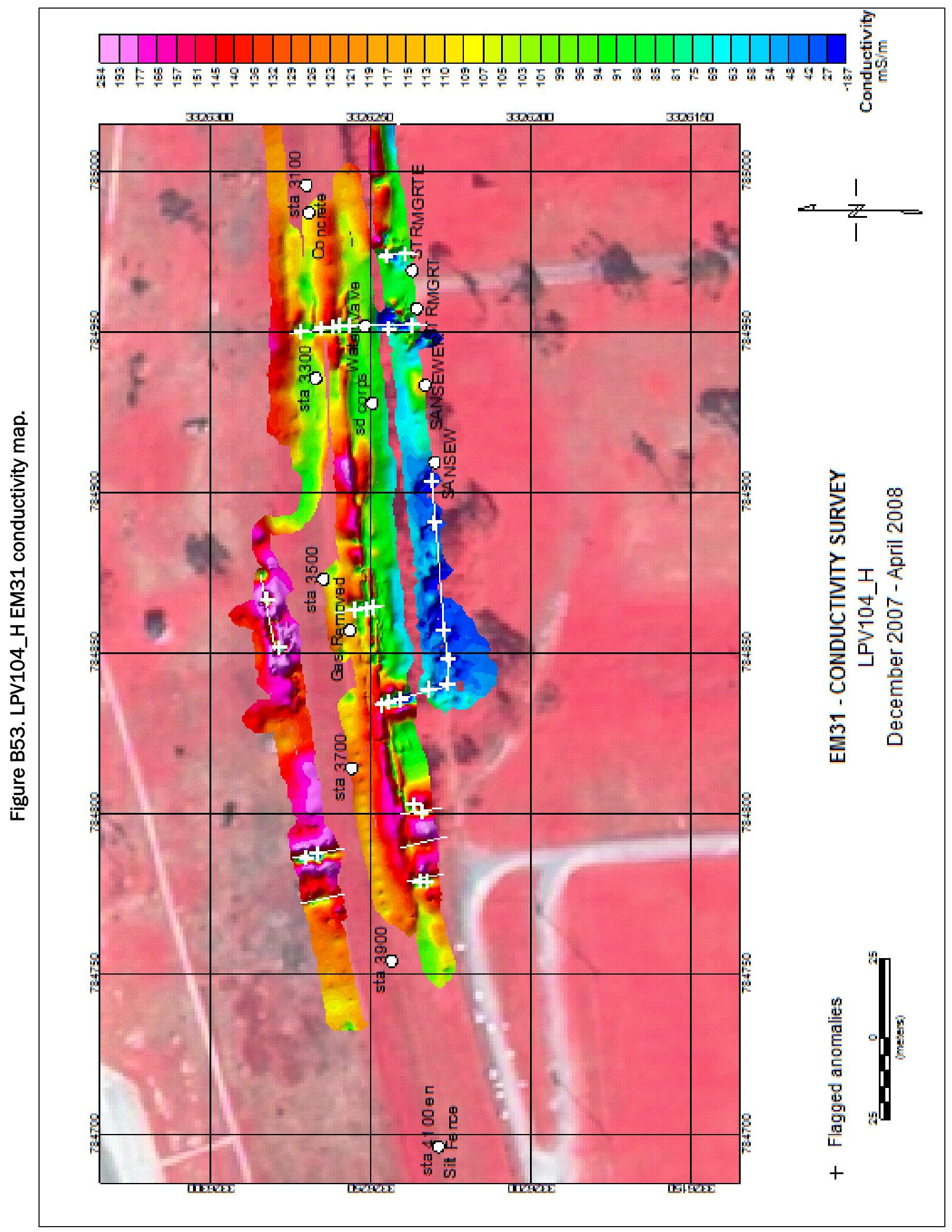




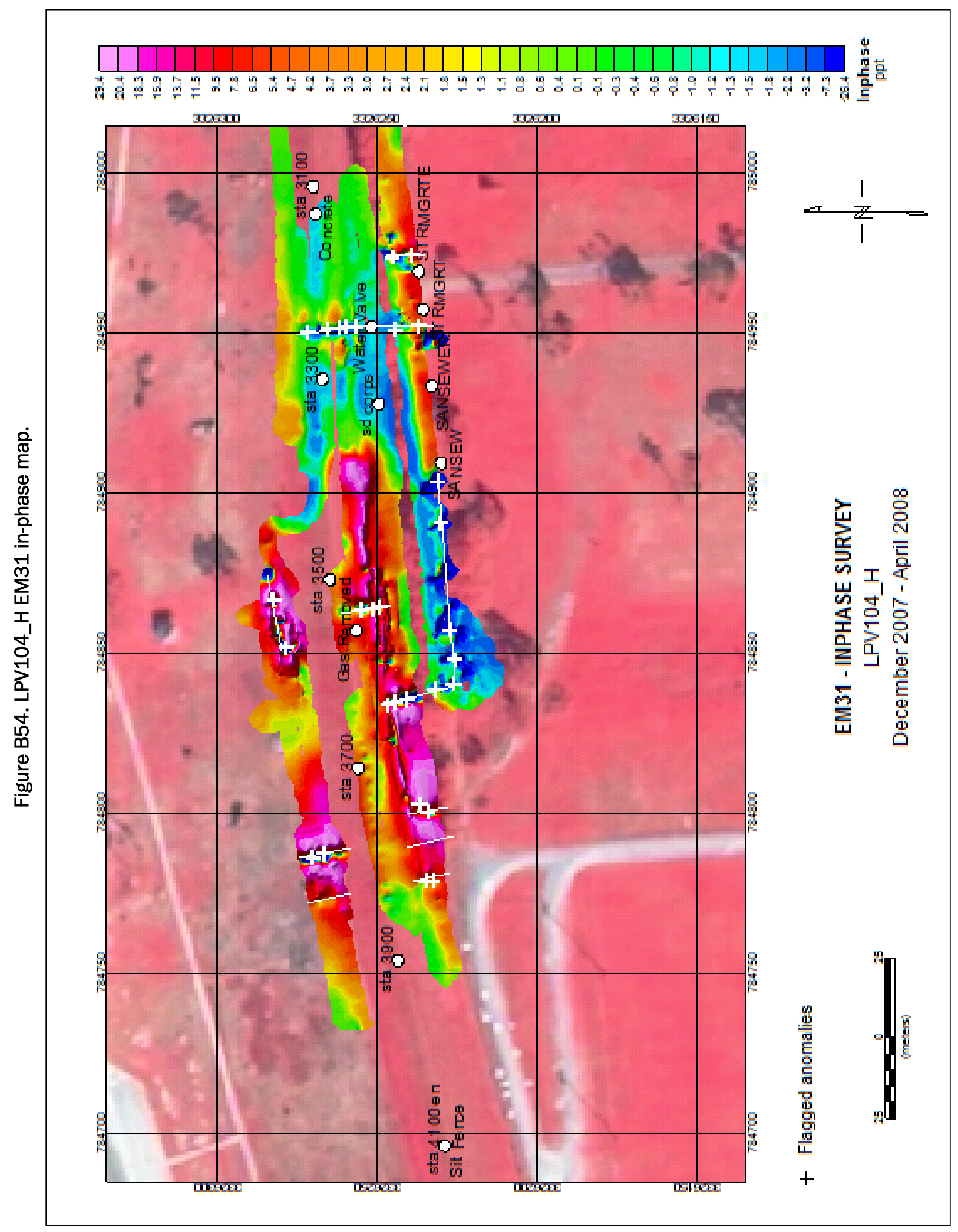




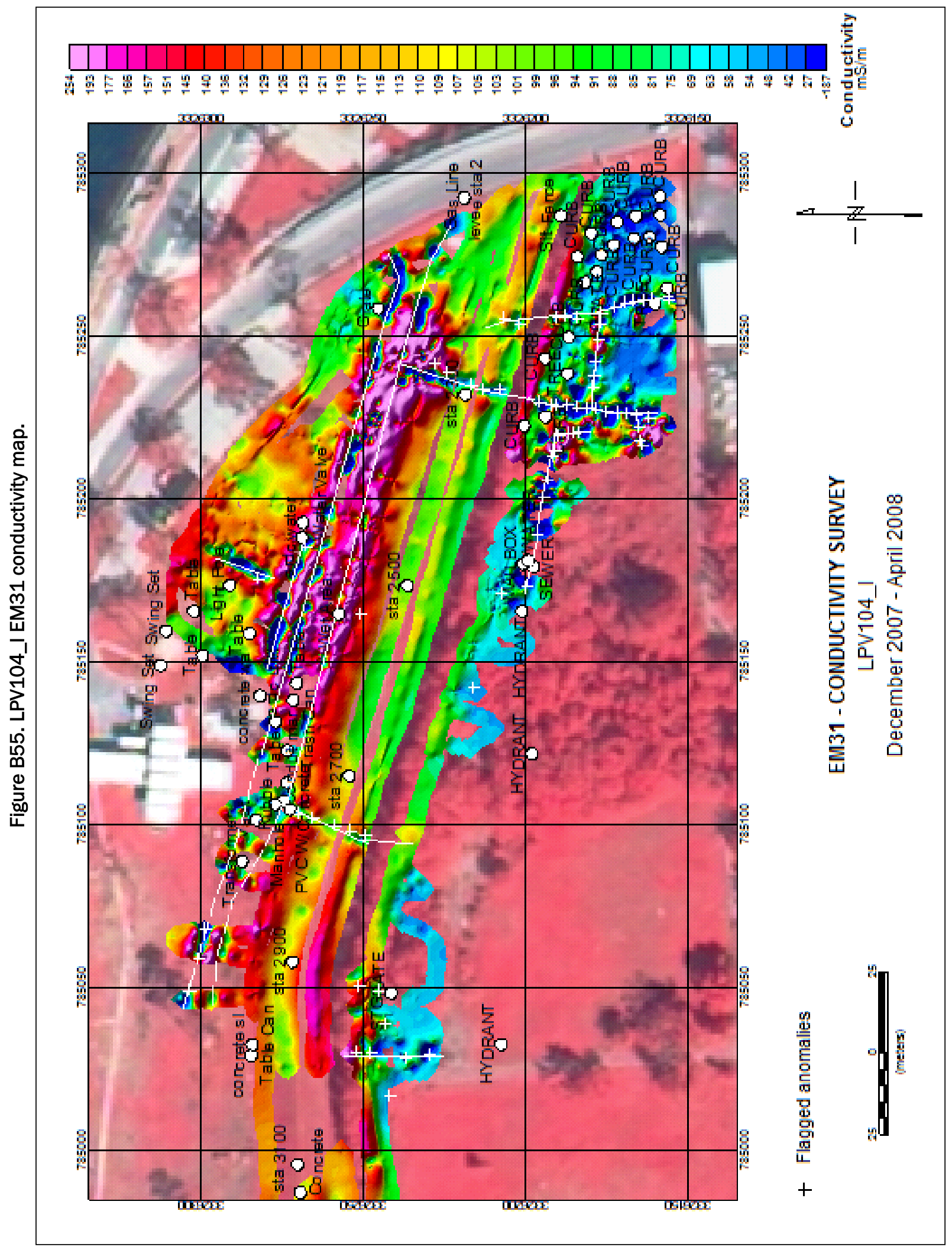




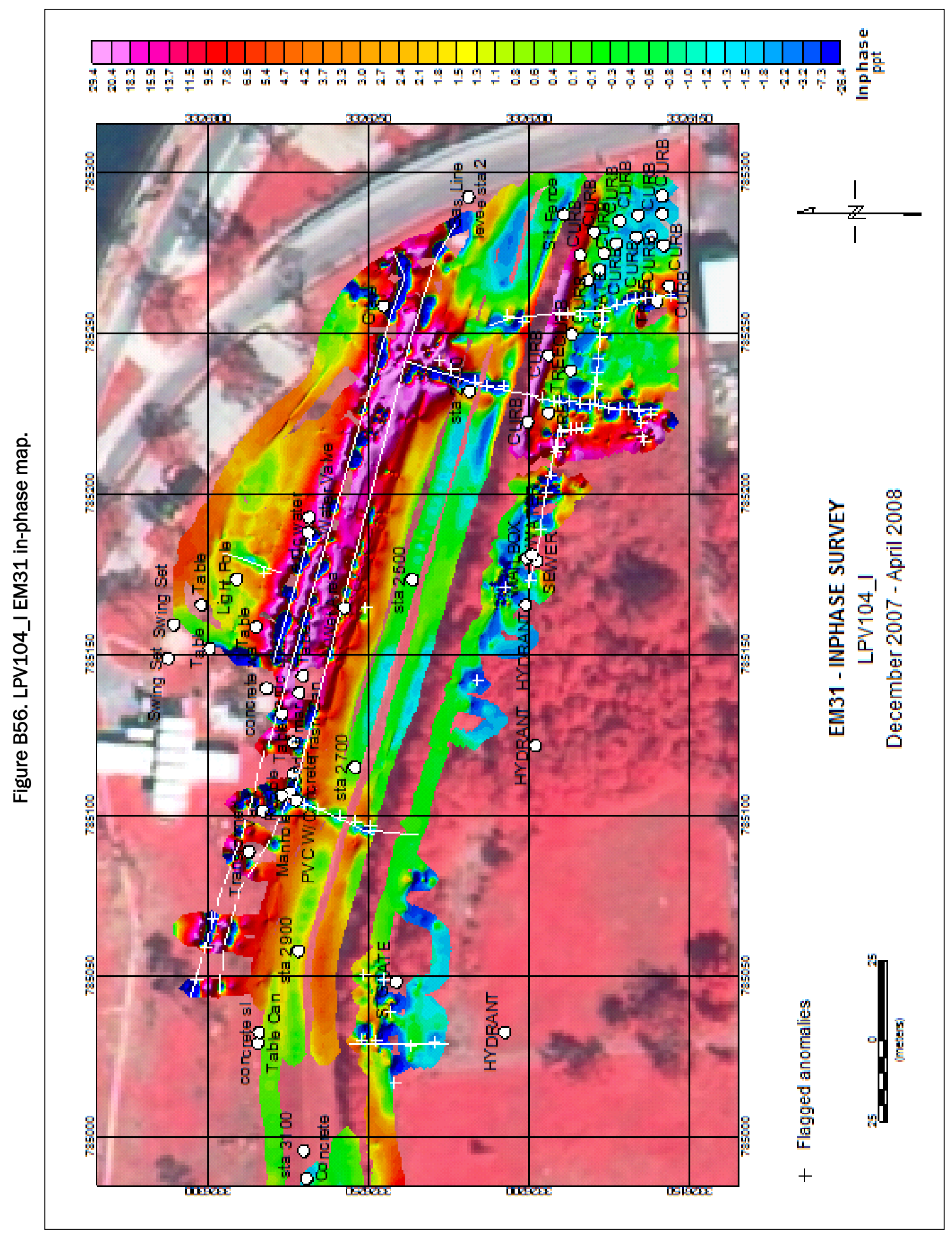


Figure B57. LPV104_J EM31 conductivity map.

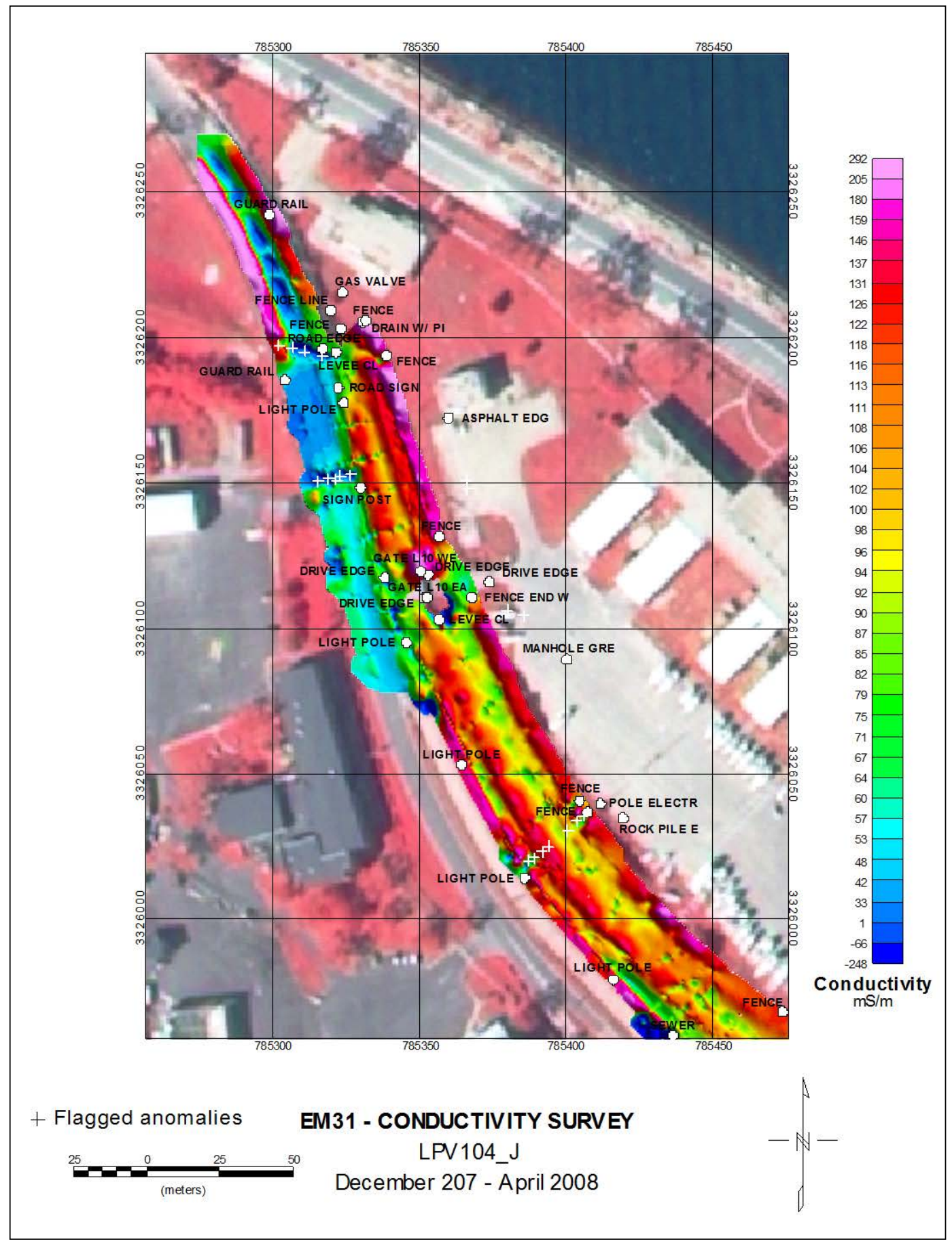


Figure B58. LPV104_J EM31 in-phase map.

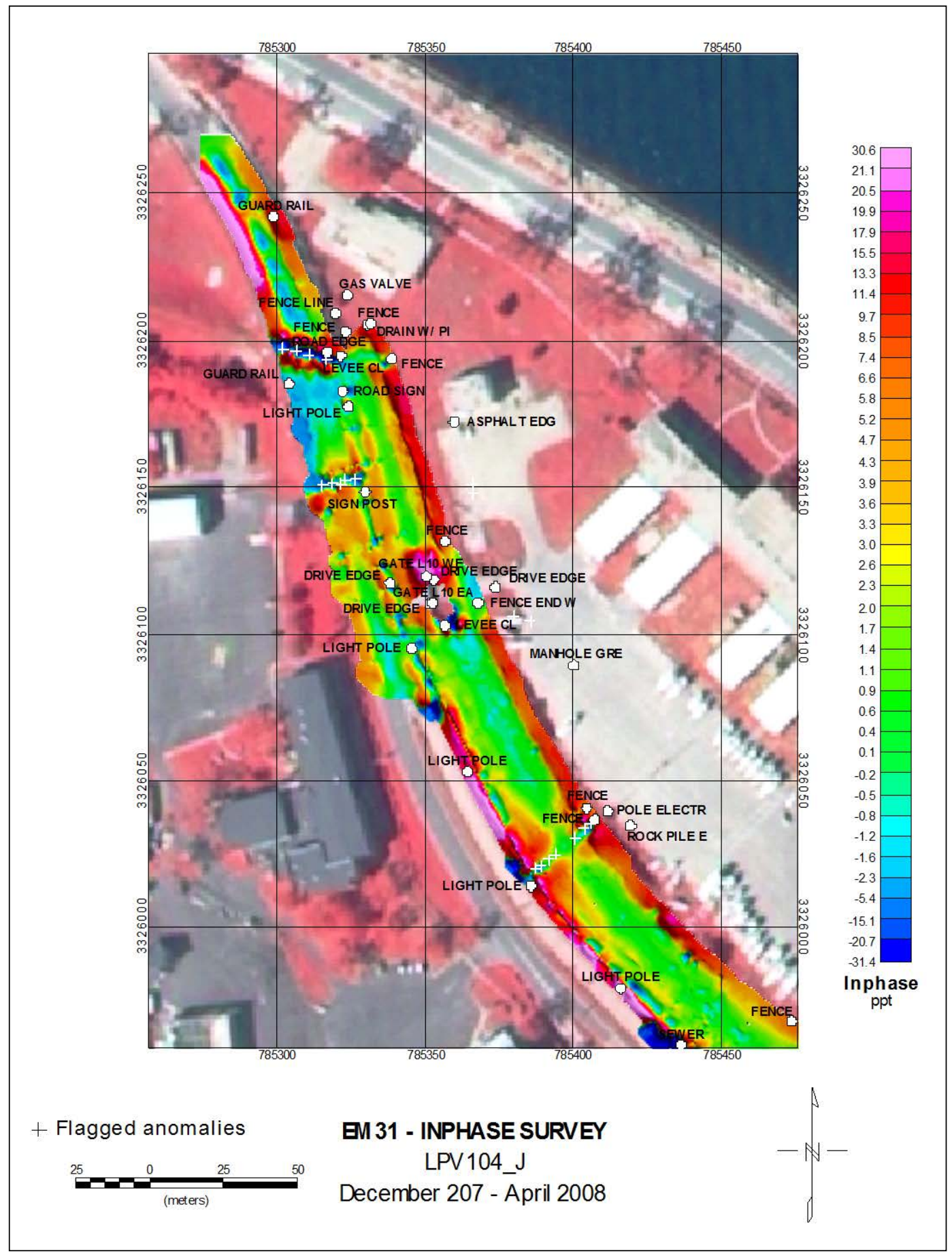




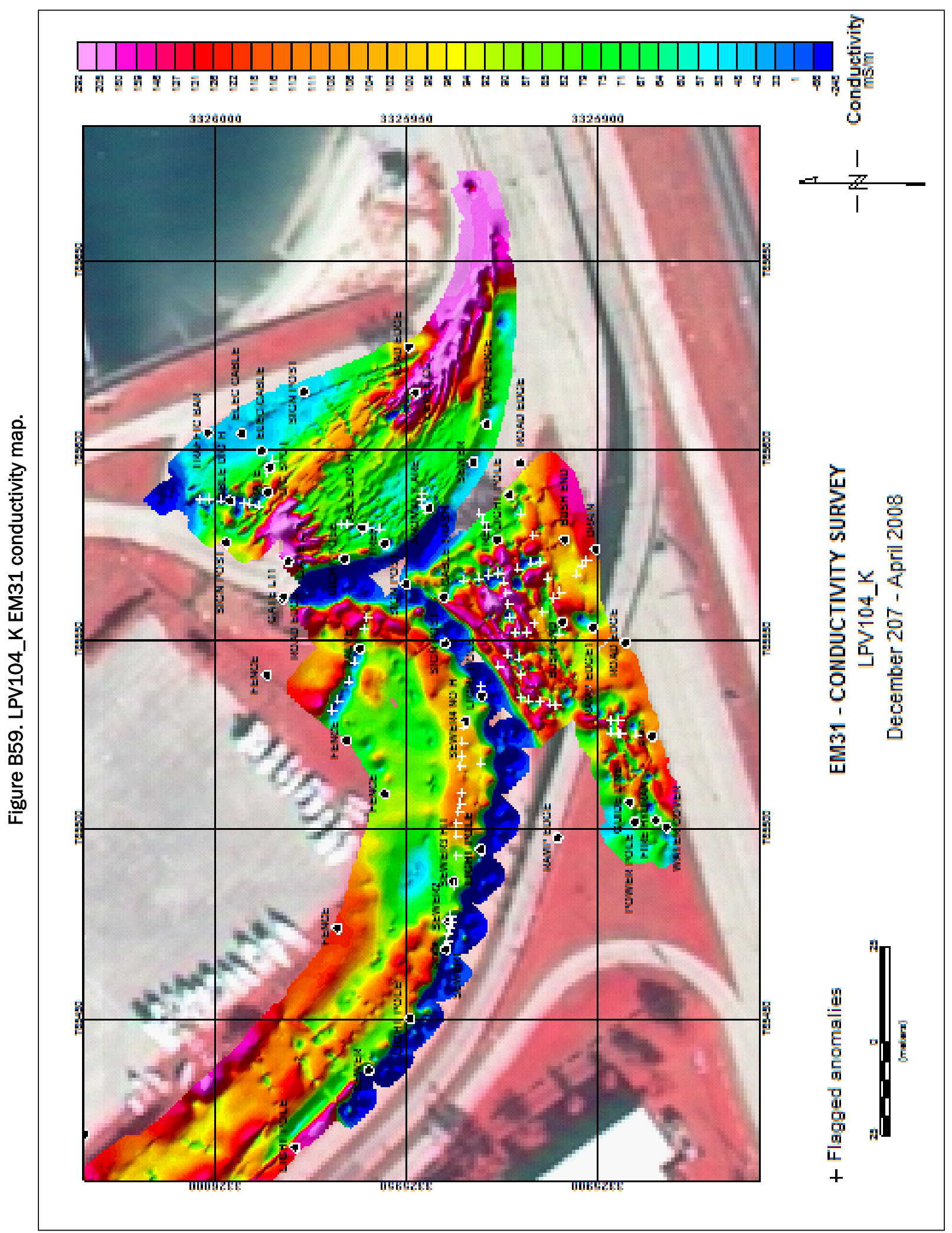




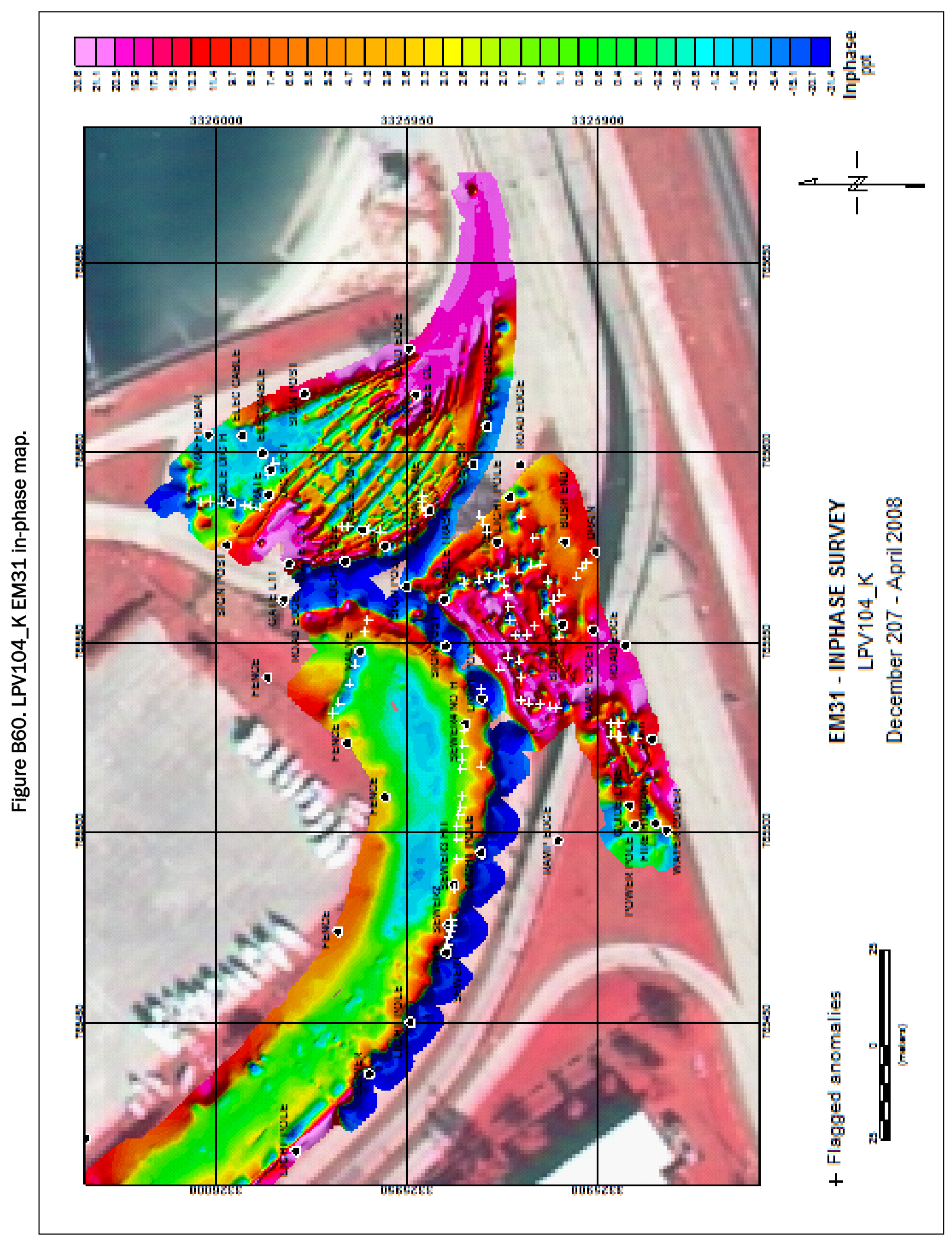




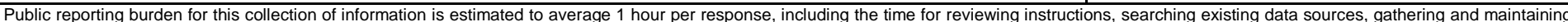

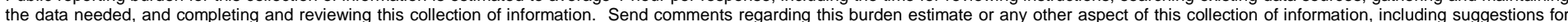

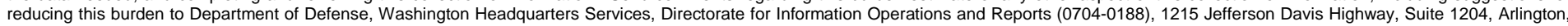

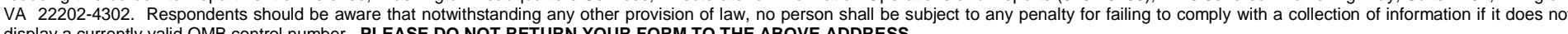
display a currently valid OMB control number. PLEASE DO NOT RETURN YOUR FORM TO THE ABOVE ADDRESS.
1. REPORT DATE (DD-MM-YYYY)
June 2014

\section{TITLE AND SUBTITLE}

Geophysical Surveys for Locating Buried Utilities, Lake Pontchartrain Levees, New Orleans

6. AUTHOR(S)

José L. Llopis, Janet E. Simms, and Grant A. Riddick
3. DATES COVERED (From - To)

5a. CONTRACT NUMBER

5b. GRANT NUMBER

5c. PROGRAM ELEMENT NUMBER

5d. PROJECT NUMBER

5e. TASK NUMBER

5f. WORK UNIT NUMBER

8. PERFORMING ORGANIZATION REPORT NUMBER

ERDC/GSL TR-14-23

10. SPONSOR/MONITOR'S ACRONYM(S)

11. SPONSOR/MONITOR'S REPORT NUMBER(S)

Approved for public release; distribution is unlimited.

\section{SUPPLEMENTARY NOTES}

\section{ABSTRACT}

This report presents the results of a geophysical study performed to determine the location of buried utilities beneath or in the vicinity of the levees on the south shore of Lake Pontchartrain approximately $8 \mathrm{~km}$ (5 miles) north of downtown New Orleans, LA. There was concern that utilities located beneath or buried near the toe of the levees could act as a water conduit during flooding events. If a waterfilled utility fails, it is possible that it may cause the levee to fail either by piping material from within the levee or cause slope stability problems. It is also possible that buried utilities can act as potential seepage paths through the levee during high water events. In this case, the buried pipe would not have to "fail" to cause a problem. The utilities needed to be accurately located so that they could be rerouted, removed, or abandoned and grouted-in. Electromagnetic, total field magnetic, and ground penetrating radar systems were assessed to determine the best method for detecting the buried utilities in this area. The Geonics EM31 electromagnetic induction instrument was considered the most effective for detecting the utilities. EM31 anomalies, presumed to be the locations of buried utilities, were mapped and their coordinates tabulated for further interrogation.

\begin{tabular}{lll}
\hline 15. SUBJECT TERMS & Utilities & Electromagnetic induction (EM) \\
Geophysics & New Orleans & Magnetic \\
Levee & Electromagnetic & Ground penetrating radar (GPR)
\end{tabular}

\section{SECURITY CLASSIFICATION OF:}

a. REPORT

UNCLASSIFIED

b. ABSTRACT
UNCLASSIFIED

c. THIS PAGE

UNCLASSIFIED

17. LIMITATION
OF ABSTRACT

18. NUMBER
OF PAGES
174

19a. NAME OF RESPONSIBLE PERSON

19b. TELEPHONE NUMBER (include area code) 